\title{
HEIGHTS OF PROJECTIVE VARIETIES AND POSITIVE GREEN FORMS
}

\author{
J.-B. BOST, H. GILLET, AND C. SOULÉ
}

\section{CONTENTS}

Introduction

1. Preliminaries: Green forms of log type

1.1. Forms and currents on complex varieties

1.2. Construction of Green forms

1.3. Operations on Green currents

1.4. An application: Levine forms and comparison of norms on polynomials

1.5. The continuity of some fiber integrals

2. Preliminaries: Arithmetic intersection theory

2.1. Arithmetic Chow groups and Chern classes

2.1.1. Arithmetic Chow groups

2.1.2. Arithmetic Chern classes

2.1.3. The degree maps on $\widehat{C H}^{*}\left(\operatorname{Spec}\left(\mathscr{O}_{K}\right)\right)$

2.2. The intersection product

2.3. The pairing $\widehat{C H}^{*}(X) \times Z_{*}(X) \rightarrow \widehat{C H}^{*}\left(\operatorname{Spec}\left(\mathscr{O}_{K}\right)\right)_{\mathbb{Q}}$

2.3.1. Definition and basic properties

2.3.2. Arakelov varieties and arithmetic fundamental classes

2.3.3. External products

2.3.4. Degrees

3. The height of cycles defined by an hermitian line bundle

3.1. Definition and examples

3.1.1. The height $h_{\bar{L}}$

3.1.2. Examples

3.1.3. Multiheights

3.1.4. Change of ground ring

3.2. Properties of $h_{\bar{L}}$

3.2.1. Basic identities

3.2.2. Dependence on metrics and integral structures

3.2.3. Positivity

Received by the editors April 4, 1993.

1991 Mathematics Subject Classification. Primary 14G25, 14C15.

The second author was partially supported by N.S.F. grant DMS-9203379. 
3.2.4. Finiteness

3.2.5. Hilbert-Samuel formula

3.3. More examples

3.3.1. Faltings heights of hypersurfaces and of projective spaces

3.3.2. Heights of linear projections

3.3.3. The Arakelov-Faltings invariant $\left(\bar{\omega}_{X / S} \cdot \bar{\omega}_{X / S}\right)$

3.3.4. Heights on abelian varieties

4. The height of cycles in projective spaces

4.1. The projective height

4.1.1. Definition

4.1.2. First properties and examples

4.2. The height of joins

4.2.1. Arithmetic integral geometry

4.2.2. Joins

4.2.3. A Bézout theorem for heights

4.3. The height of Chow forms and resultants

4.3.1. Definitions

4.3.2. Remarks anả examples

4.3.3. The height of generalized Chow divisors

4.3.4. The height of generalized Chow forms

5. Positivity of the projective height and arithmetic Bézout theorem

5.1. The basic inequality

5.2. Lower bounds for the projective height

5.3. The fundamental class of an intersection

5.4. Arithmetic Bézout theorem

5.4.1. Computations in $C H^{*}(\overline{\mathbb{P}(E)})$

5.4.2. Arithmetic Bézout theorem

5.4.3. Remarks

5.5. Arithmetic Bézout theorem for improper intersections

5.6. Variants

6. Positive Green forms

6.1. Positive Green forms and the Bézout theorem

6.2. Construction of positive Green forms

6.3. An obstruction to the existence of positive Green forms

\section{INTRODUCTION}

Chaque proposition dans Bezout a l'air d'un grand secret appris d'une bonne femme voisine.

Stendhal, Vie de Henry Brulard.

The purpose of this paper is to study analogs of some basic concepts and results of projective geometry in the context of Arakelov geometry [Ar2, G-S2].

As was first noticed by Faltings in his work on diophantine approximation for abelian varieties [Fa2], higher dimensional arithmetic intersection theory can be used to define the height of any (closed integral) projective subscheme $X \subset \mathbb{P}^{N}$, where $\mathbb{P}^{N}$ is the $N$-dimensional projective space over $\mathbb{Z}$ (or more generally over the integers in a number field). The Faltings height $h_{F}(X)$, which 
is a nonnegative real number, is defined in a similar fashion to the degree of a projective variety over a field. That is, $h_{F}(X)$ is the intersection, in the sense of [G-S2], of the fundamental class of $X$ with the first Chern class of the canonical hermitian line bundle on $\mathbb{P}^{N}$, raised to the power $d=\operatorname{dim}(X)$ (see 3.1 below).

In this paper we propose a slightly different definition of the height of $X$. Namely we denote by $h(X)$ the intersection of the fundamental class of $X$ with the $d$-th Chern class of the canonical quotient hermitian bundle on $\mathbb{P}^{N}$ (see Definition 4.1.1). We prove that $h(X)$ is nonnegative and smaller than $h_{F}(X)$ (except when $d \leq 1$ or when the generic fiber of $X$ is empty, in which case $\left.h(X)=h_{F}(X)\right)$. Furthermore $h(X)=0$ if and only if $X$ is a linear subspace $\mathbb{P}^{d-1} \subset \mathbb{P}^{N}$ defined by the vanishing of $N+1-d$ standard coordinates (Theorem 5.2.3).

We obtain several results on the heights of projective varieties, which are inspired by the analogy between heights and degrees. For instance we compute the height of the join of two varieties (Proposition 4.2.2) and the behavior of the height under linear projection (3.3.2). We give several proofs of the following arithmetic Bézout theorem. Assume $X \subset \mathbb{P}^{N}$ and $Y \subset \mathbb{P}^{N}$ are integral projective varieties which meet properly on the generic fiber of $\mathbb{P}^{N}$. Their intersection cycle $X . Y$ can then be defined using Fulton's method [Fu2]. It is well defined up to the addition of a cycle linearly equivalent to zero in the closed fibers of $\mathbb{P}^{N}$ over $\mathbb{Z}$, and its height $h(X . Y)$ (defined by extending by linearity the definition for integral subschemes) does not depend on the choice of representative for $X . Y$. Denote by $\operatorname{deg}_{\mathbb{Q}}(X)$ and $\operatorname{deg}_{\mathbb{Q}}(Y)$ the degrees in $\mathbb{P}_{\mathbb{Q}}^{N}$ of the generic fibers of $X$ and $Y$ respectively. Then we have

$$
h(X . Y) \leq h(X) \operatorname{deg}_{\mathbb{Q}}(Y)+\operatorname{deg}_{\mathbb{Q}}(X) h(Y)+c \operatorname{deg}_{\mathbb{Q}}(X) \operatorname{deg}_{\mathbb{Q}}(Y),
$$

where the constant $c$ depends only on $N, \operatorname{dim}(X)$, and $\operatorname{dim}(Y)$ (see also [Fa2] in the case of complete intersections). We give three different proofs of this inequality (Theorems 4.2.3, 5.4.4, and 6.1.1), the smallest value of $c$ being the one in Theorem 5.4.4 (we believe that $c$ can be taken equal to zero, but we cannot prove it except when $X$ or $Y$ is a linear subspace).

In transcendental number theory, especially in the work of Nesterenko [N1], [N2] and Philippon [P1], another definition of height has been known for some time, which does not use Arakelov theory, and cases of the Bézout theorem have been proved in that context. Namely the height of $X \subset \mathbb{P}^{N}$ is defined to be the height of its Chow form, which is a point in a large projective space. The comparison between this definition and $h_{F}(X)$ was made by Soule [So2] and Philippon [P1]. We extend their result to more general Chow forms and not necessarily standard metrics (Theorem 4.3.2). As a byproduct we get the following result. Let $R$ be the resultant of $N+1$ homogeneous polynomials of degrees $d_{0}, \ldots, d_{N}$ in $N+1$ variables. This is a multihomogeneous polynomial with integral coefficients of multidegree $\left(\delta_{0}, \ldots, \delta_{N}\right)$, where $\delta_{i}=\prod_{\substack{j=0 \\ j \neq i}}^{N} d_{j}$. Its variables are the coefficients of the "generic" homogeneous polynomials of degrees $d_{0}, \ldots, d_{N}$ in $N+1$ variables. So $R$ can be viewed as an element of 
$\bigotimes_{i=0}^{r} S^{\delta_{i}}\left(S^{d_{i}} \mathbb{C}^{\stackrel{\vee}{N+1}}\right)^{\vee}$. Equip this vector space with the hermitian norm $\|\cdot\|_{\text {Herm }}$ induced by the standard hermitian structure on $\mathbb{C}^{N+1}$. We prove in Lemma 4.3.4 that

$$
\begin{aligned}
\log \|R\|_{\text {Herm }}= & \frac{1}{2}\left(\prod_{i=0}^{N} d_{i}\right) \cdot\left((N+1)\left(1+\frac{1}{2}+\cdots+\frac{1}{N}\right)-N\right) \\
& +\varepsilon\left(d_{0}, \ldots, d_{N}\right),
\end{aligned}
$$

where

$$
\left|\varepsilon\left(d_{0}, \ldots, d_{N}\right)\right| \leq \frac{1}{2} N\left(\prod_{i=0}^{N} d_{i}\right) \cdot \sum_{i=0}^{N} \frac{1}{d_{i}} \log \left(d_{i}+1\right) .
$$

We also evaluate the size of $R$ for other norms (Theorem 4.3.8).

Our main analytic tool is the existence of "positive Green forms" for effective cycles $Z$ on a complex manifold $X$. By this we mean a positive $C^{\infty}$ form $\eta$ on $X-|Z|$ which is locally $L^{1}$ on $X$ and such that the corresponding current $g=[\eta]$ on $X$ is a Green current for $Z$, i.e., such that $d d^{c} g+\delta_{Z}$ is $C^{\infty}$ on $X$ (where $\delta_{Z}$ is the current given by integration on $Z$ ). An example of such a positive Green form is the Levine form, familiar to Nevanlinna theory [Lev], [St2], when $X$ is a complex projective space and $Z$ a linear subspace. The positivity of these Levine forms has several interesting consequences (Proposition 1.4.2, Proposition 4.1.3). More generally we give conditions for a given effective cycle (resp. all effective cycles) on $X$ to have a positive Green form (Propositions 6.2.1, 6.2.2, and 6.2.3), and a counterexample showing that some complex manifolds admit effective cycles with no positive Green forms (6.3).

The paper is organized as follows. In Section 1 we discuss Green forms "of $\log$ type" for cycles on complex varieties. A general construction is given in 1.2, together with examples. The star product of Green currents is described in 1.3. These two sections (as well as the beginning of Section 2) cover material from [G-S2], but they improve it in several ways. For instance the associativity of the star product is shown in Theorem 1.3.2 without assuming that the ambient variety is projective. In 1.4 we use the positivity of Levine forms to compare several norms on polynomials, and in 1.5 we prove the continuity of some fiber integrals. This last result is used in Section 5 to get arithmetic inequalities, but it is probably of independent interest.

In 2.1 we discuss arithmetic intersection theory, and in 2.2 we explain why the intersection product is invariant under linear equivalence. A pairing between algebraic and arithmetic cycles is discussed in 2.3, and Proposition 2.3.1 describes its basic properties. This pairing (which was independently considered by Zhang [ $\mathrm{Zh} 2])$ is the main tool to define heights.

Section 3 studies the height attached to an hermitian line bundle (see also [Zh2] and [Gu]). A special case is the height introduced by Faltings in [Fa2]. We compute how this height varies with the data (3.2.2), we give conditions for it to be nonnegative (3.2.3), and we prove the finiteness of any set of cycles whose height and degree with respect to some ample hermitian line bundle are bounded (3.2.4). In 3.2.5 we give a formula for this height analogous to the Hilbert-Samuel formula for the degree. Section 3.3 is devoted to examples: 
heights of hypersurfaces (3.3.1), behavior of the height under linear projection (3.3.2, see also [Fa2]), self-intersection of the dualizing sheaf on arithmetic surfaces (3.3.3), heights of subvarieties of abelian varieties (3.3.4; we interpret Philippon's height [P2] as the height defined by some hermitian line bundle, see also $[\mathrm{Kr}]$ and $[\mathrm{Gu}])$.

In Section 4 we define the height $h(X)$. Propositions 4.1.2 and 4.1.3 contain some of its properties and the comparison with $h_{F}(X)$. In Proposition 4.2.2 we compute $h(X \# Y)$, where $X \# Y$ is the join of two projective varieties $X$ and $Y$. This leads in Theorem 4.2.3 to a first proof of the arithmetic Bézout theorem. In 4.3 we compute the height of generalized Chow forms (Theorems 4.3.2 and 4.3.8) and we deduce the estimates of resultants mentioned above. Note that in many respects the height $h(X)$ appears as the dual notion, for projective duality, of the Faltings height $h_{F}(X)$.

In Section 5, we use the analytic result from 1.5 to prove that $h(X) \geq 0$ (Theorem 5.2.3; we also consider the case of nonstandard projective spaces) and to get our best version of the arithmetic Bézout theorem (Theorem 5.4.4). We also prove some Bézout theorems for cycles which do not meet properly on the generic fiber (Theorem 5.5.1), and variants involving more than two cycles or several projective spaces (5.6). It would be of interest to get precise analogs of the Bézout theorem with excess of Vogel [V] and Fulton ([Fu2], Chapter XII).

The last section is devoted to the study of positive Green forms. We get from them a third proof of Bézout's theorem (Theorem 6.1.1), we discuss cases where they exist (6.2), and where they don't (6.3).

Some of the results in this paper were announced in the note [BGS]. We thank O. Gabber for helpful comments and for the proof of Proposition 6.2.3.

Conventions. The following notations are used throughout this paper.

When $X$ is a scheme and $E$ a vector bundle on $X$, we let $\mathscr{E}$ be the locally free $\mathscr{O}_{X}$-module of sections of $E, \mathscr{E}^{\vee}=\operatorname{Hom}\left(\mathscr{E}, \mathscr{O}_{X}\right)$ its dual, $S^{k}\left(\mathscr{E}^{\vee}\right)$ its symmetric powers, and

$$
\mathbb{P}(E)=\operatorname{Proj}\left(\underset{k \geq 0}{\oplus} S^{k}\left(\mathscr{E}^{\vee}\right)\right)
$$

the associated projective scheme; i.e., $\mathbb{P}(E)=\mathbb{P}\left(\mathscr{E}^{\vee}\right)$ in Grothendieck's notation. The canonical quotient line bundle on $\mathbb{P}(E)$ is denoted $\mathscr{O}_{E}(1)$. When $E$ is a holomorphic vector bundle on a complex space $X$ we define similarly $\mathbb{P}(E)$ and $\mathscr{O}_{E}(1)$.

When $p \geq 0$ is an integer and $X$ a scheme of finite type over a Dedekind ring we let $\bar{Z}_{p}(X)$ be the group of algebraic cycles of dimension $p$ on $X$. If $X$ is equidimensional we let

$$
Z^{p}(X)=Z_{\operatorname{dim}(X)-p}(X)
$$

From the beginning of Section 2, we let $K$ be a number field, $\mathscr{O}_{K}$ be its ring of integers, and $S=\operatorname{Spec}\left(\mathscr{O}_{K}\right)$ be the associated affine scheme; all arithmetic varieties are $S$-schemes, and their products are products over $S$. 


\section{Preliminaries: Green fORMS OF LOG TYPE}

\subsection{Forms and currents on complex varieties.}

1.1.1. If $X$ is a complex manifold, we shall denote by $A^{p q}(X)\left(\right.$ resp. $\left.\mathscr{D}^{p q}(X)\right)$ the vector space of complex-valued $C^{\infty}$ differential forms (resp., of complexvalued currents, i.e., of differential forms with distribution coefficients) of type $(p, q)$. Thanks to the canonical orientation of $X$ (defined by the volume form $d x_{1} \wedge d y_{1} \wedge \cdots \wedge d x_{d} \wedge d y_{d}$ for any choice of local holomorphic coordinates $\left.z_{\alpha}=x_{\alpha}+i y_{\alpha}, \alpha=1, \ldots, d\right)$, the space $A^{p q}(X)$ may be identified with a subspace of $\mathscr{D}^{p q}(X)$. More generally, any locally $L^{1}$ form $\varphi$ of type $(p, q)$ on $X$ defines a current on $X$, that we shall sometimes denote by $[\varphi]$ to avoid ambiguity.

If $Y \subset X$ is a closed irreducible analytic subset of codimension $p$, we denote by $\delta_{Y}$ the current of integration on $Y$; it is the current in $\mathscr{D}^{p, p}(X)$ which maps any compactly supported smooth form $\eta$ on $X$ to its integral on the smooth part of $Y$. For any desingularization $\nu: \widetilde{Y} \rightarrow Y$ of $Y$, this integral coincides with $\int_{\widetilde{Y}} \nu^{*} \eta$. In other words

$$
\delta_{Y}=\nu_{*}[1]
$$

By linearity, the definition of $\delta_{Y}$ is extended to any analytic cycle on $X$.

A Green current for an analytic cycle $Z$ of codimension $p$ in $X$ is an element $g \in \mathscr{D}^{p-1, p-1}(X)$ such that

$$
d d^{c} g+\delta_{Z} \in A^{p p}(X) .
$$

Here $d=\partial+\bar{\partial}, d^{c}=(i / 4 \pi)(\bar{\partial}-\partial)$, and therefore $d d^{c}=(i / 2 \pi) \partial \bar{\partial}$.

A Green form for $Z$ is a Green current which is locally $L^{1}$ on $X$ and $C^{\infty}$ on $X-|Z|$.

\subsubsection{Recall from [G-S2], 1.3.2, the following definition.}

Definition 1.1.1. Let $X$ be a smooth quasi-projective complex variety and $Y$ a proper closed algebraic subset of $X$. A $C^{\infty}$ form $\eta$ on $X-Y$ is said to be of log type along $Y$ when there exist a smooth quasi-projective complex variety $M$ (nonnecessarily connected), a proper morphism $\pi: M \rightarrow X$, and a $C^{\infty}$ form $\varphi$ on $M-\pi^{-1}(Y)$ such that:

(i) $\pi^{-1}(Y)$ is a divisor with normal crossings, and $\pi$ is smooth over $X-Y$;

(ii) $\eta$ is the direct image by $\pi$ of $\varphi_{\mid Z-\pi^{-1}(Y)}$;

(iii) For any point $x \in M$, there is an open neighborhood $U$ of $x$ and a system of holomorphic coordinates $\left(z_{1}, \ldots, z_{n}\right)$ of $U$ centered at $x$ such that the set $\pi^{-1}(Y) \cap U$ has equation $z_{1} \cdots z_{k}=0$, for some $k \leq n$, and there exist smooth $\partial$ - and $\bar{\partial}$-closed forms $\alpha_{i}$ on $U, i=1, \ldots, k$, and a smooth form $\beta$ on $U$ with $\varphi_{\mid U}=\sum_{i=1}^{k} \alpha_{i} \log \left|z_{i}\right|^{2}+\beta$.

Such a form $\eta$ on $X-Y$ is locally $L^{1}$ on $X$ and defines a current [ $\left.\eta\right]$ on $X$, which coincides with the direct image $\pi_{*}[\varphi]$ of the current $[\varphi]$ defined by $\varphi$ (cf. [G-S2], 1.1.5). 
Examples. (i) If $L$ is an (algebraic) line bundle on $X$, endowed with a $C^{\infty}$ hermitian metric \|\| , and if $s$ is a regular section of $L$ on $X$ which does not vanish identically on any component of $X$, then $\log \|s\|$ is a 0 -form of log type along the divisor $Y$ of $s$. This follows from Hironaka's Theorem on embedded resolution applied to $Y \subset X$.

(ii) Suppose $Y$ is a smooth subvariety of $X$. Let $\nu: \tilde{X} \rightarrow X$ be the blow-up of $Y$ in $X$ and $E=\nu^{-1}(Y)$ the exceptional divisor of $\nu, s$ a regular section of $\mathscr{O}(E)$ with divisor $E$. For any $C^{\infty}$ hermitian metric \|\| on $E$ and any $C^{\infty}$ forms $\alpha$ and $\beta$ on $\widetilde{X}$ such that $\alpha$ is $\partial$ - and $\bar{\partial}$-closed, the $C^{\infty}$ form $\log \|s\| . \alpha+\beta$ on $\widetilde{X}-X \simeq X-Y$ is of log type along $Y$. (Take $M=\tilde{X}$ and $\pi=\nu$.)

Forms on $X$ of log type along $Y$ are easily seen to form a vector space stable under multiplication by $\partial$ - and $\bar{\partial}$-closed $C^{\infty}$ forms on $X$. The following is proved in [G-S2], 1.3.3 and 2.1.4, by using resolution of singularities.

Lemma 1.1.2. Let $X$ and $X^{\prime}$ be smooth quasi-projective complex varieties, and let $\eta$ be a $C^{\infty}$ form on $X-Y$ of log type along a closed algebraic subset $Y \subset X$.

(i) For any morphism $f: X^{\prime} \rightarrow X$ such that $f^{-1}(Y)$ does not contain any component of $X^{\prime}$, the form $f^{*} \eta$ on $X^{\prime}-f^{-1}(Y)$ is of log type along $f^{-1}(Y)$.

Suppose moreover that $Y$ is the support of a cycle $Z$ of codimension $p$ on $X$ and that $[\eta]$ is a Green current for $Z$. Then, if $f^{-1}(Y)$ has codimension $p$ in $X^{\prime},\left[f^{*} \eta\right]$ is a Green form for the cycle $f^{*}(Z)$ on $X^{\prime}$ that we shall denote by $f^{*}[\eta]$; more precisely, if we let

$$
\omega=d d^{c}[\eta]+\delta_{Z} \in A^{p p}(X)
$$

then

$$
d d^{c}\left[f^{*} \eta\right]+\delta_{f^{*}(Z)}=f^{*}(\omega)
$$

(ii) For any proper morphism $f: X \rightarrow X^{\prime}$ which is smooth outside $Y$ and such that $f(Y)$ does not contain any component of $X^{\prime}$, the $C^{\infty}$ form $f_{*} \eta$ on $X^{\prime}-f(Y)$ is of log type along $f(Y)$.

Observe that, in (ii), the currents $f_{*}[\eta]$ and $\left[f_{*} \eta\right]$ coincide (cf. [G-S2], 1.1.5). If $Z$ is any irreducible subvariety of $X$ which is not contained in $Y$, any $C^{\infty}$ form $\eta$ on $X-Y$ of $\log$ type along $Y$ is locally $L^{1}$ with respect to the current of order zero $\delta_{Z}$, and the product $\eta \delta_{Z}$ is a well-defined current of order 0 . In fact, for any resolution $\nu: \widetilde{Z} \rightarrow Z$, the form $\nu^{*} \eta$ is locally $L^{1}$ in $\tilde{Z}$ and

$$
\eta \delta_{Z}=\nu_{*}\left[\nu^{*} \eta\right]
$$

This extends by linearity to arbitrary algebraic cycles $Z$ on $X$. 
1.1.3. We now recall a few basic facts concerning positive forms on complex manifolds. ${ }^{1}$

Definition 1.1.3. A smooth form $\eta$ of type $(p, p)$ on a complex manifold $X$ is said to be a positive form if for any complex manifold $V$ of complex dimension $p$ and any holomorphic map $\varphi: V \rightarrow X$, the volume form $\varphi^{*} \eta$ on $X$ is nonnegative.

Clearly, in this definition, we can restrict $V$ to be a polydisc in $\mathbb{C}^{p}$. The positivity of $\eta$ will be denoted: $\eta \geq 0$.

A real $C^{\infty}$ form $\omega$ of type $(1,1)$ on $X$ may be written in terms of local holomorphic coordinates $\left(z_{1}, \ldots, z_{d}\right)$ as

$$
\omega=\sum_{k, \ell=1}^{d} \omega_{k \ell} d z_{k} \wedge d \bar{z}_{\ell}
$$

where $\omega_{k \ell}+\bar{\omega}_{\ell k}=0$. It is positive iff the associated hermitian form $h$ on $T_{X}$, defined as

$$
h=\frac{1}{i} \sum_{k, \ell=1}^{d} \omega_{k \ell}\left(d z_{k} \otimes d \bar{z}_{\ell}+d z_{\ell} \otimes d \bar{z}_{k}\right),
$$

is nonnegative. If $h$ is a positive definite hermitian form, then $\omega$ is said to be strictly positive.

In the sequel, we shall just use the following properties of positive forms:

Proposition 1.1.4. Let $M$ and $N$ be complex manifolds, and let $\eta$ be a $C^{\infty}$ positive form of type $(k, k)$ on $M$.

(i) For any holomorphic map $f: N \rightarrow M$, the smooth form $f^{*} \eta$ is positive on $N$.

(ii) If $g: M \rightarrow N$ is a smooth holomorphic map whose restriction to the support of $\eta$ is proper, the smooth form $g_{*} \eta$ is positive on $N$.

(iii) For any $C^{\infty}$ positive form $\omega$ of type $(1,1)$ on $M$, the form $\omega . \eta$ is positive.

(iv) Let $\omega$ be a strictly positive $C^{\infty}$ form of type $(1,1)$ on $M$. For any $C^{\infty}$ form $\alpha$ of type $(p, p)$ on $M$ and any relatively compact open subset $\Omega \subset M$, there exists a real number $R$ such that for any $t \geq R$, the $(p, p)$-form $\alpha+t \omega^{p}$ is positive on $\Omega$.

Proof. Assertion (i) follows immediately from the definition.

To prove (ii), we may assume that $k \geq d:=\operatorname{dim}_{\mathbb{C}} N-\operatorname{dim}_{\mathbb{C}} M$. For any complex manifold $V$ of dimension $k-d$ and any holomorphic map $\varphi: V \rightarrow$ $N$, we can consider the following cartesian diagram of complex manifolds:

\footnotetext{
${ }^{1}$ The reader should be aware that, for forms of type $(k, k)$ on a $d$-dimensional complex manifold, there are (at least!) three natural notions of positivity, which are distinct when $2 \leq k \leq d-2$; the definition which we use in this paper coincides with the one introduced by Lelong [Le1], and is sometimes called "weak positivity" (see [Ha-K]).
} 


$$
\begin{aligned}
& W:=V \times_{N} M \stackrel{\Phi}{\longrightarrow} M \\
& \downarrow G \quad \downarrow g \\
& V \underset{\varphi}{\longrightarrow} N \text {. }
\end{aligned}
$$

Then the identity

$$
\varphi^{*} g_{*} \eta=G_{*} \Phi^{*} \eta
$$

shows that $\varphi^{*} g_{*} \eta$ is the direct image by a smooth map of a nonnegative volume form, hence nonnegative (compare [St3], Theorem AII 5.4).

For a proof of (iii) see [Le1], IV, Proposition 3, or [Ha-K].

Assertion (iv) can be shown by a compactness argument.

If $X$ is a complex manifold and $Z$ an analytic cycle on $X$, a Green form $g$ for $Z$ whose restriction to $X-|Z|$ is positive will be called a positive Green form for $Z$. Suppose that $X$ is quasi-projective and that $Z$ is an algebraic cycle. For any such form $g$ of type $(p, p)$ which is log type along $|Z|$ and any effective algebraic cycle $Z^{\prime}$ of dimension $p$ on $X$, no component of which is contained in $|Z|$, it follows from (1.1.2) and Proposition 1.1.4, (iv) that the current $g . \delta_{Z^{\prime}}$ is a positive measure on $X$. In particular, if $X$ is projective, we get:

$$
\int_{X} g \delta_{Z^{\prime}} \geq 0 .
$$

\subsection{Construction of Green forms.}

1.2.1. Let $X$ be a complex manifold, $Y \subset X$ a closed complex submanifold of codimension $p, \nu: \widetilde{X} \rightarrow X$ the blow-up of $Y$ in $X$, and $E=\nu^{-1}(Y)$ its exceptional divisor, so that we have a diagram:

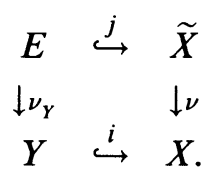

We shall also denote by $N$ the normal bundle to $Y$ in $X$, and by $Q$ the canonical quotient bundle $\nu_{Y}^{*} N / \mathscr{O}_{N}(-1)$ on the projective bundle $\mathbb{P}(N)$, which may be identified with $E$. Finally we choose a $C^{\infty}$ hermitian metric $\|\cdot\|$ on $\mathscr{O}_{\widetilde{X}}(E)$, and a holomorphic section $s$ of $\mathscr{O}_{\widetilde{X}}(E)$ on $\widetilde{X}$ with divisor $E$, and we let

$$
\beta=c_{1}\left(\mathscr{O}_{\widetilde{X}}(E),\|\|\right) .
$$

It is a closed form in $A^{1,1}(\tilde{X})$, which satisfies the following identities of currents: 


$$
d d^{c} \log \|s\|^{-2}+\delta_{E}=\beta .
$$

In this section, we shall denote by $[c]$ the cohomology class of a closed current $c$.

Lemma 1.2.1. Let $\alpha \in A^{p-1, p-1}(X)$ be a closed form such that the cohomology class of $j^{*}(\alpha)$ is the $(p-1)$-th Chern class $c_{p-1}(Q) \in H^{2 p-2}(E ; \mathbb{C})$.

(i) The following equalities hold:

$$
\nu_{*}\left(\alpha \delta_{E}\right)=\delta_{Y} \in \mathscr{D}^{p-1, p-1}(X)
$$

and

$$
\nu^{*}\left[\delta_{Y}\right]=[\alpha \wedge \beta] \in H^{2 p}(\tilde{X}) .
$$

(ii) Suppose moreover that $\omega \in A^{p, p}(X)$ is a closed form such that $[\omega]=\left[\delta_{Y}\right]$ in $H^{2 p}(X ; \mathbb{C})$ and that $\gamma \in A^{p-1, p-1}(X)$ is such that

$$
d d^{c} \gamma=\nu^{*}(\omega)-\alpha \wedge \beta
$$

Then the current

$$
g=\nu_{*}\left[\log \|s\|^{-2} \cdot \alpha+\gamma\right] \in \mathscr{D}^{p-1, p-1}(X)
$$

satisfies the following identity:

$$
d d^{c} g+\delta_{Y}=\omega
$$

In particular, $g$ is a Green current for $Y$. Observe that $g$ is locally $L^{1}$ on $X$ and $C^{\infty}$ on $X-Y$, and that, if $X$ is a quasi-projective variety and $Y$ an algebraic subvariety, $g$ is log type along $Y$ (cf. 1.1.2, Example (ii)).

Proof (Compare [G-S2], 1.3.6 and 1.3.7). (i) We have

$$
\nu_{*}\left(\alpha \delta_{E}\right)=\nu_{*}\left(\alpha . j_{*} 1\right)=\nu_{*}\left(j_{*} j^{*} \alpha\right)=i_{*} \nu_{Y_{*}} j^{*} \alpha .
$$

As $\nu_{Y}$ is proper and smooth, the current $\nu_{Y_{*}} j^{*} \alpha$ belongs to $A^{00}(Y)$ and is defined by the function whose value at $y \in Y$ is

$$
\int_{\nu_{Y}^{-1}(y)} j^{*} \alpha=\int_{\mathbb{P}\left(N_{y}\right)} \alpha=\left\langle\left[\mathbb{P}\left(N_{y}\right)\right], c_{p-1}(Q)\right\rangle=1 .
$$

Therefore

$$
\nu_{*}\left(\alpha \delta_{E}\right)=i_{*}(1)=\delta_{Y} .
$$

By the "key formula" ([Fu2], Proposition 6.7. (a)), we get the equality of cohomology classes 


$$
\nu^{*}\left[\delta_{Y}\right]=j_{*} c_{p-1}(Q)
$$

According to (1.2.1) and the hypothesis made in $\alpha$, we have

$$
\left[\delta_{E}\right]=[\beta] \text { and } c_{p-1}(Q)=j^{*}[\alpha] .
$$

Therefore

$$
\nu^{*}\left[\delta_{Y}\right]=j_{*} j^{*}[\alpha]=[\alpha] j_{*}[1]=[\alpha]\left[\delta_{E}\right]=[\alpha \wedge \beta] .
$$

(ii) Since $\alpha$ is $\partial$ - and $\bar{\partial}$-closed, we have

$$
d d^{c} g=\nu_{*}\left(d d^{c}\left(\log \|s\|^{-2}\right) \cdot \alpha+d d^{c} \gamma\right) .
$$

Equation (1.2.6) now follows immediately from (1.2.1), (1.2.2), and (1.2.4).

Lemma 1.2.1 is the basic tool for constructing Green forms, as will be shown in the next two subsections.

1.2.2. Suppose moreover that $X$ is a compact Kähler manifold. Then so is $\widetilde{X}$ [BI] and the conditions in Lemma 1.2.1, (ii) are always satisfied: the existence of $\omega$ follows from Hodge theory and that of $\gamma$ from the $\partial \bar{\partial}$-lemma ([G-S2], 1.2.1). Therefore, if there is a closed form $\alpha \in A^{p-1, p-1}(X)$ satisfying the hypothesis of Lemma 1.2.1, there exists a Green form for $Y$ (of log type along $Y$ in the quasi-projective case). By Hodge theory again, this condition amounts to:

$$
c_{p-1}(Q) \in j^{*} H^{2 p-2}(\widetilde{X} ; \mathbb{C})
$$

Since

$$
c_{p-1}(Q)=\sum_{i=0}^{p-1} \nu_{Y}^{*} c_{p-1-i}(N) c_{1}\left(\mathscr{O}_{N}(1)\right)^{i},
$$

this happens when the total Chern class $c(N)$ lies in the image of the restriction map

$$
i^{*}: H^{*}(X ; \mathbb{C}) \rightarrow H^{*}(Y ; \mathbb{C})
$$

Consider now a smooth projective variety $M$. Both $M$ and $M \times M$ are Kähler, and the map (1.2.7) is surjective when $Y=\Delta$ is the diagonal in $X=$ $M \times M$. Therefore there exists a Green form $g_{\Delta}$ for $\Delta$ in $M \times M$, of log type along $\Delta$. Starting from $g_{\Delta}$, one easily gets Green forms for any cycle in $M$. Indeed, if we denote $\mathrm{pr}_{i}: M \times M \rightarrow M(i=1,2)$ as the two projections, we have (compare [B1], Theorem 2.1):

Lemma 1.2.2. For any cycle $Z \in Z_{p}(M)$, the current

$$
g=p r_{1 *}\left(g_{\Delta} \cdot p r_{2}^{*} \delta_{Z}\right)
$$

is a Green form for $Z$, of log type along $|Z|$. 
In (1.2.8), the current $g_{\Delta} \cdot p r_{2}^{*} \delta_{Z}$ is well defined as the product of $g_{\Delta}$ and $p r_{2}^{*} \delta_{Z}=\delta_{M \times Z}$, since $\Delta$ and $M \times Z$ meet properly (cf. 1.1).

Proof. We may assume that $Z$ is an irreducible subvariety, and consider a resolution $\nu: \widetilde{Z} \rightarrow Z$. Let

$$
\mu=\left(\mathrm{id}_{M}, \nu\right): M \times \widetilde{Z} \rightarrow M \times Z,
$$

and let $p: M \times \widetilde{Z} \rightarrow M$ be the first projection. By definition

$$
g_{\Delta} \cdot p r_{2}^{*} \delta_{Z}=\mu_{*}\left[\mu^{*} g_{\Delta}\right]
$$

Therefore

$$
g=p r_{1 *} \mu_{*}\left[\mu^{*} g_{\Delta}\right]=p_{*}\left[\mu^{*} g_{\Delta}\right]
$$

This shows that the current $g$ is $L^{1}$ on $M$ (cf. [G-S2], 1.1.5) and $C^{\infty}$ on $M-|Z|$. Moreover it follows from Lemma 1.1.2 that it is log type along $|Z|$. Finally, if we let

$$
\begin{gathered}
\omega_{\Delta}=d d^{c} g_{\Delta}+\delta_{\Delta} \\
\left(\in A^{d-1, d-1}(M \times M), d=\operatorname{dim} M\right),
\end{gathered}
$$

we get from Lemma 1.1.2, (i):

$$
d d^{c}\left[\mu^{*} g_{\Delta}\right]=\mu^{*} \omega_{\Delta}-\delta_{\mu^{*} \Delta} ;
$$

on the other hand, we have the equality of cycles

$$
p_{*} \mu^{*} \Delta=Z \text {, }
$$

hence the equality of currents (cf. [G-S2], p.136)

$$
p_{*} \delta_{\mu^{*} \Delta}=\delta_{Z}
$$

From (1.2.9) and (1.2.10), we obtain

$$
d d^{c} g+\delta_{Z}=p_{*} \mu^{*} \omega_{\Delta} .
$$

As $p$ is a smooth map, this current is $C^{\infty}$.

Observe that, as a corollary of the previous discussion, we recover the fact that, for any cycle $Z$ on a smooth projective variety $M$, there exists a Green form for $Z$ of $\log$ type along $|Z|$ ([G-S2], 1.3.8-1.3.9). Using the existence of a smooth projective compactification for any smooth quasi-projective complex variety, one sees that this still holds when $M$ is only assumed to be quasiprojective. 
1.2.3. Suppose now that there exists a holomorphic vector bundle $F$ of rank $p$ on the complex manifold $X$ such that $Y$ is the set of zeros of some global holomorphic section $\sigma$ of $F$ on $X$ which is transverse to the zero section. If we write $\mathscr{I}$ for the sheaf of ideals in $\mathscr{O}_{X}$ of functions vanishing on $Y$, we have an epimorphism:

$$
\sigma^{*}: \mathscr{F}^{\vee} \rightarrow \mathscr{I} \subset \mathscr{O}_{X},
$$

where $\mathscr{F}$ is the sheaf of holomorphic sections of $F$ and $\mathscr{F}^{\vee}$ is its dual. If $i$ is the inclusion of $Y$ into $X$, this induces an isomorphism

$$
i^{*} \mathscr{F}^{\vee} \simeq \mathscr{I} / \mathscr{F}^{2}
$$

and hence

$$
N_{Y / X} \simeq i^{*} F \text {. }
$$

The homomorphism $\sigma^{*}$ also induces an epimorphism of graded algebras of $\mathscr{O}_{X}$-modules

$$
\underset{k \geq 0}{\oplus} \operatorname{Sym}^{k}\left(\mathscr{F}^{\vee}\right) \rightarrow \underset{k \geq 0}{\oplus} \mathscr{I}^{k}
$$

and hence a closed immersion:

$$
\begin{array}{ccc}
f: \widetilde{X} & \longrightarrow & \mathbb{P}(F) \\
\| & \| \\
\operatorname{Proj}\left(\underset{k \geq 0}{\oplus} \mathscr{I}^{k}\right) & \operatorname{Proj}\left(\underset{k \geq 0}{\oplus} \operatorname{Sym}^{k}\left(\mathscr{F}^{\vee}\right)\right),
\end{array}
$$

for which

$$
f^{*} \mathscr{O}_{F}(1)=\mathscr{O}_{\widetilde{X}}(1)=\mathscr{I} \mathscr{O}_{\widetilde{X}}=\mathscr{O}_{\widetilde{X}}(-E) .
$$

The map $f$ fits into the following commutative diagram:

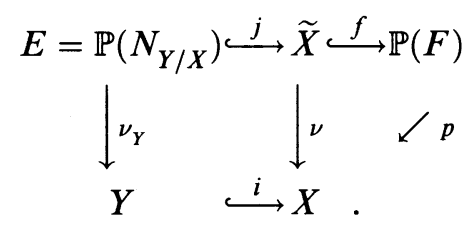

The composition, where $s: \mathscr{O}_{\widetilde{X}} \hookrightarrow \mathscr{O}_{\widetilde{X}}(E)$ is the canonical section,

$$
\nu^{*} \mathscr{F}^{*} \longrightarrow f^{*} \mathscr{O}_{\mathbb{P}(F)}(1)=\mathscr{O}_{\widetilde{X}}(-E) \stackrel{s^{*}}{\longrightarrow} \mathscr{O}_{\widetilde{X}}
$$


is $\nu^{*}\left(\sigma^{*}\right)$. In the dual sequence

$$
\mathscr{O}_{\widetilde{X}} \stackrel{s}{\longrightarrow} \mathscr{O}_{\widetilde{X}}(E) \longleftrightarrow \nu^{*} \mathscr{F},
$$

the composite map is $\nu^{*}(\sigma)$. On the open set $\widetilde{X}-E=X-Y$, where $\nu$ is the identity, it coincides with $\sigma$.

Suppose now that $F$ is endowed with a $C^{\infty}$ hermitian metric. This metric determines hermitian metrics on $p^{*} F$, on its quotient bundle $Q_{F}$, on its subbundle $\mathscr{O}_{F}(-1)$, and therefore on $\mathscr{O}_{\widetilde{X}}(E)$. Let $c_{k}(\bar{F}), c_{k}\left(\bar{Q}_{F}\right), \ldots$ denote the Chern forms associated with these metrics. These are closed $C^{\infty}$ forms of type $(k, k)$, whose cohomology classes are the usual Chern classes $c_{k}(F)$, $c_{k}\left(Q_{F}\right), \ldots$. Moreover the closed form

$$
p^{*} c_{p}(\bar{F})-c_{1}\left(\overline{\mathscr{O}_{F}(-1)}\right) \cdot c_{p-1}\left(\bar{Q}_{F}\right) \in A^{p, p}(\mathbb{P}(F)),
$$

whose cohomology class vanishes, may be written $d d^{c} \eta$, where $\eta$ lies in $A^{p-1, p-1}(\mathbb{P}(F))$. This follows from the results of Bott and Chern [B-C], $\S 5$, applied to the following exact sequence of hermitian vector bundles:

Also observe that

$$
\mathscr{E}: 0 \rightarrow \mathscr{O}_{F}(-1) \rightarrow p^{*} F \rightarrow Q_{F} \rightarrow 0 .
$$

$$
\beta=c_{1}(\overline{\mathscr{O}(E)})=f^{*} c_{1}\left(\overline{\mathscr{O}_{F}(-1)}\right) \text { and }\|s\|^{2}=\nu^{*}\|\sigma\|^{2} .
$$

We conclude that the various conditions in Lemma 1.2.1 are satisfied by

$$
\alpha=f^{*} c_{p-1}\left(\bar{Q}_{F}\right), \omega=c_{p}(\bar{F}), \text { and } \gamma=f^{*} \eta,
$$

and finally, we get that

$$
g=\nu_{*}\left(\nu^{*} \log \|\sigma\|^{-2} \cdot f^{*} c_{p-1}\left(\bar{Q}_{F}\right)+f^{*}(\eta)\right)
$$

is a Green form for $Y$ in $X$, such that

$$
d d^{c} g+\delta_{Y}=c_{p}(\bar{F}) \text {. }
$$

It is of log type along $Y$ when $X$ is quasi-projective, and $F$ and $\sigma$ are algebraic.

Examples. (i) Suppose $X$ is the total space of a holomorphic vector bundle $\pi: \xi \rightarrow Y$ over $Y$. Then $Y$ may be defined by the vanishing of the tautological section $\sigma$ of $F:=\pi^{*} \xi$, and the previous construction defines, for any choice of an hermitian metric on $\xi$ and of the form $\eta$, a Green current for $Y$. As a matter of fact, the general construction may be recovered from this special case: the Green form (1.2.11) coincides with the pull-back by $\sigma: X \rightarrow F$ of the Green form of $X$ in the total space of $F$ obtained from the tautological section of the pull-back of $F$. This remark allows one to extend formula (1.2.11) to the case where $\sigma$ is only supposed to meet properly the zero section of $F$ and $Y$ is the cycle attached to the l.c.i. subscheme defined by the vanishing of $\sigma$. 
(ii) Consider the special case of (i) where $Y$ is a point and $\xi=\mathbb{C}^{p}$ is equipped with the standard metric

$$
\|z\|^{2}=\sum_{i=1}^{p}\left|z_{i}\right|^{2} .
$$

Then $\mathbb{P}(F)=\mathbb{C}^{p} \times \mathbb{P}^{p-1}(\mathbb{C})$ and the exact sequence of hermitian vector bundles $\mathscr{E}$ on $\mathbb{P}(F)$ is the pull back of the analogous exact sequence on $\mathbb{P}^{p-1}(\mathbb{C})$. Thus

$$
c_{1}\left(\overline{\mathscr{\sigma}_{F}(-1)}\right) \cdot c_{p-1}\left(\bar{Q}_{F}\right)-f^{*} c_{p}(\bar{F})=0
$$

and we may choose $\eta=0$. Therefore

$$
g=\nu_{*}\left(\log \|s\|^{-2} \cdot f^{*} c_{p-1}\left(\bar{Q}_{F}\right)\right)
$$

is a Green form of log type for the origin in $\mathbb{C}^{p}$. In terms of coordinates

$$
g=-\log \|z\|^{2} \cdot\left(d d^{c} \log \|z\|^{2}\right)^{p-1} \quad \text { on } \mathbb{C}^{p}-\{0\},
$$

and the identity

$$
d d^{c} g=-\delta_{\{0\}}
$$

is essentially the Bochner-Martinelli formula.

(iii) Suppose $X$ is a Stein manifold. Then there exists a Stein neighborhood $\Omega$ of $Y$ in its normal bundle $N$ and a "tubular neighborhood" map $\varphi: \Omega \rightarrow X$, i.e., an open holomorphic immersion such that $\varphi_{\mid Y}=\mathrm{id}_{\mid Y}$. According to (i), there exists a Green form $g$ for $Y$ in $N$. For any $\rho \in A^{00}(X)$ such that

$$
\rho \equiv 1 \text { near } Y \text { and } \operatorname{supp} \rho \subset \varphi(\Omega),
$$

the current $\rho \varphi_{*}(g)$ on $X$ is a Green form for $Y$ in $X$. This shows that any smooth cycle on $X$ has a Green form.

A reduction to the diagonal analogous to Lemma 1.2.2, the details of which we leave to the reader, shows that the same is true for any analytic cycle on $X$.

(iv) Formula (1.2.11) gives in particular a Green form for any smooth complete intersection $Y$. Indeed, if $Y=\bigcap_{i=1}^{p} H_{i}$, where $H_{i}$ are (closed) complex hypersurfaces in $X$ which are smooth and meet transversally along $Y$, and if $s_{i}$ is a section $\mathscr{O}\left(H_{i}\right)$ with divisor $H_{i}$, we may apply the construction above to the section $s=\left(s_{i}\right)_{1 \leq i \leq p}$ of $F=\bigoplus_{i=1}^{p} \mathscr{O}\left(H_{i}\right)$. Using the last observation in (i), this may be extended to any complete intersection.

(v) Let $V$ be a complex vector space, equipped with an hermitian scalar product with associated norm \|\| , and $\mathbb{P}(V)$ the complex projective space of lines in $V$. For any linear subspace $W \subset V$ of codimension $p>0$, consider the subvariety $\mathbb{P}(W) \subset \mathbb{P}(V)$, and the vector bundle $F=\mathscr{O}_{V}(1) \otimes V / W$ on $\mathbb{P}(V)$. For any line $L \in \mathbb{P}(V)$, the fiber $F_{L}$ may be identified with the vector 
space $\operatorname{Hom}(L, V / W)$, and the section $\sigma$ of $F$ which takes as value at $L$ the composition of the tautological linear maps

$$
L \hookrightarrow V \rightarrow V / W
$$

is a regular section of $F$, which meets transversally the zero section of $F$ exactly along $\mathbb{P}(W)$. Therefore the construction above applies to $X=\mathbb{P}(V)$ and $Y=\mathbb{P}(W)$.

The projective bundle $\mathbb{P}(F)$ may be identified with $\mathbb{P}(V) \times \mathbb{P}(V / W)$, the map $p$ with the first projection (onto $\mathbb{P}(V)$ ), and $Q_{F}$ with $p^{*} \mathscr{O}_{V}(1) \otimes q^{*} Q_{V / W}$, where $q$ is the second projection onto $\mathbb{P}(V / W)$ and $Q_{V / W}$ the canonical quotient bundle on $\mathbb{P}(V / W)$; then the exact sequence $\mathscr{E}$ coincides with the pull-back by $q$ of the canonical exact sequence

$$
0 \rightarrow \mathscr{O}_{V / W}(-1) \rightarrow V / W \rightarrow Q_{V / W} \rightarrow 0
$$

twisted by $p^{*} \mathscr{O}_{V}(1)$. The hermitian scalar product on $V$ determines hermitian structures on $V / W$, on $\mathscr{O}_{V}(1)$ (which is a subbundle of $\mathscr{O}_{\mathbb{P}(V)} \otimes \stackrel{\vee}{V}$ ), on $F$ (by tensor product), on $\mathscr{O}_{V / W}(-1)$, on $Q_{V / W}$, and on $Q_{F}$. These are compatible with the isomorphisms mentioned above. It follows that

$$
\begin{gathered}
c_{p}(\bar{F})=c_{1}\left(\overline{\mathscr{O}_{V}(1)}\right)^{p} \\
c_{1}\left(\overline{\mathscr{O}_{F}(-1)}\right)=p^{*} c_{1}\left(\overline{\mathscr{O}_{V}(1)}\right)-q^{*} c_{1}\left(\overline{\mathscr{O}_{V / W}(1)}\right) \\
c_{p-1}\left(\bar{Q}_{F}\right)=\sum_{i+j=p-1} p^{*} c_{1}\left(\overline{\mathscr{O}_{V}(1)}\right)^{i} \cdot q^{*} c_{1}\left(\overline{\mathscr{O}_{V / W}(1)}\right)^{j} .
\end{gathered}
$$

Therefore

$$
p^{*} c_{p}(\bar{F})-c_{1}\left(\overline{\mathscr{O}_{F}(-1)}\right) \cdot c_{p-1}\left(\bar{Q}_{F}\right)=0
$$

and the construction above applies with $\eta=0$. Therefore the current

$$
\Lambda_{\mathbb{P}(W)}:=\nu_{*}\left(\nu^{*} \log \|\sigma\|^{-2} \cdot \sum_{i+j=p-1} \nu^{*} c_{1}\left(\overline{\mathscr{\sigma}_{V}(1)}\right)^{i} \cdot f^{*} q^{*} c_{1}\left(\overline{\mathscr{\sigma}_{V / W}(1)}\right)^{j}\right)
$$

is a Green form for $\mathbb{P}(W)$, of log type along $\mathbb{P}(W)$, which satisfies

$$
d d^{c} \Lambda_{\mathbb{P}(W)}+\delta_{\mathbb{P}(W)}=c_{1}\left(\overline{\mathscr{O}_{V}(1)}\right)^{p} .
$$

This current is called the Levine form of $\mathbb{P}(W)$ ([Lev], [G-S3], $\S 5$ ), and may be rewritten in a slightly more explicit way as follows. Let $W^{\perp}$ be the orthogonal complement to $W$ in $V$, and let $\pi: V \rightarrow W^{\perp}$ be the orthogonal projection. On $V-\{0\}$ (resp. $V-W$ ) consider the smooth function $\rho(x)=$ $\log \|x\|^{2}$ (resp. $\tau(x)=\log \|\pi(x)\|^{2}$ ); these functions define $(1,1)$ forms $\mu=$ 
$d d^{c} \rho$ on $\mathbb{P}(V)$ and $\lambda=d d^{c} \tau$ on $\mathbb{P}(V)-\mathbb{P}(W)$, and a function $\rho-\tau$ on $\mathbb{P}(V)-\mathbb{P}(W)$. Then we have:

$$
\Lambda_{\mathbb{P}(W)}=(\rho-\tau) \sum_{i+j=p-1} \mu^{i} \lambda^{j} \quad(\text { on } \mathbb{P}(V)-\mathbb{P}(W))
$$

Indeed

$$
\rho-\tau=\log \|\sigma\|^{-2}, \mu=c_{1}\left(\overline{\mathscr{O}_{V}(1)}\right), \lambda=f^{*} q^{*} c_{1}\left(\overline{\mathscr{O}_{V / W}(1)}\right) .
$$

1.3. Operations on Green currents. Let $X$ be a smooth quasi-projective complex variety. Given two currents $S$ and $T$ on $X$, we shall write $S \equiv T$ to mean that there exist currents $u$ and $v$ on $X$ with $S-T=\partial u+\bar{\partial} v$, and we shall denote by $\widetilde{\mathscr{D}}^{p p}(X)$ the quotient space $\mathscr{D}^{p p}(X) / \equiv$ and by $\widetilde{T}$ the class in $\widetilde{\mathscr{D}}^{p p}(X)$ of a current $T \in \mathscr{D}^{p p}(X)$.

Let $Y \subset X$ be a closed irreducible subvariety, $\eta_{Y}$ a Green form for $Y$ of $\log$ type along $Y$, and $Z$ an algebraic cycle on $X$ which meets $Y$ properly. Using Lemma 1.1.2, (i), one sees that the current $\eta_{Y} \delta_{Z}$ (defined by (1.1.2)) satisfies the following equation:

$$
d d^{c}\left(\eta_{Y} \delta_{Z}\right)+\delta_{Y . Z}=\omega_{Y} \delta_{Z}
$$

where $\omega_{Y}$ is the form $d d^{c} \eta_{Y}+\delta_{Y}$ and $Y . Z$ the usual intersection cycle of $Y$ and $Z$ (compare [G-S2], proof of Theorem 2.1.4). If $g_{Z}$ is any Green current of $Z$, we define following [GS2], 2.1, the star product of $\eta_{Y}$ and $g_{Z}$ to be

$$
\eta_{Y} * g_{Z}=\eta_{Y} \delta_{Z}+\omega_{Y} g_{Z}
$$

It follows from (1.3.1) that it is a Green current for $Y . Z$.

Proposition-Definition 1.3.1. Let $Y_{1}, \ldots, Y_{k}$ be irreducible subvarieties of codimension $p>0$ in $X, n_{1}, \ldots, n_{k}$ some integers, and $Z$ the cycle $\sum_{i=1}^{k} n_{i} Y_{i} \in Z^{p}(X)$.

(i) For any Green current $g_{Z}$ for $Z$ in $X$, there exist Green forms $\eta_{Y_{i}}$ for $Y_{i}$, of log type along $Y_{i}$, such that

$$
g_{Z} \equiv \sum_{i=1}^{k} n_{i} \eta_{Y_{i}}
$$

(ii) For any algebraic cycle $Z^{\prime}$ on $X$ which meets $Z$ properly and any Green current $g_{Z^{\prime}}$ for $Z^{\prime}$, the class in $\widetilde{D}(X)$ of the current $\sum_{i=1}^{k} n_{i} \eta_{Y_{i}} \delta_{Z^{\prime}}$ (resp. of the Green current $\sum_{i=1}^{k} n_{i} \eta_{Y_{i}} * g_{Z^{\prime}}$ for $Z . Z^{\prime}$ ) depends only on $\widetilde{g}_{Z}$ and $\delta_{Z^{\prime}}$ (resp. on $\tilde{g}_{Z}$ and $\tilde{g}_{Z^{\prime}}$ ) and will be denoted $\widetilde{g}_{Z} \delta_{Z^{\prime}}$ (resp. $\left.\widetilde{g}_{Z} * \widetilde{g}_{Z^{\prime}}\right)$. 
(iii) Let $X^{\prime}$ be another smooth quasi-projective complex variety and $f$ : $X^{\prime} \rightarrow X$ a morphism such that $f^{-1}(|Z|)$ has codimension $p$ in $X^{\prime}$. The class in $\widetilde{\mathscr{D}}\left(X^{\prime}\right)$ of the Green form $\sum_{i=1}^{k} n_{i}\left[f^{*} \eta_{Y_{i}}\right]$ for $f^{*} Z$ (cf. Lemma 1.1.2, (i)) depends only on $\tilde{g}_{Z}$ and will be denoted $f^{*} \tilde{g}_{Z}$.

Proof. The existence of the $\eta_{Y_{i}}$ 's satisfying (1.3.3) follows from the existence of Green forms of log type for any subvariety of a quasi-projective variety and from the fact that, if $g$ and $g^{\prime}$ are any two Green currents for $Z$, there exists a $C^{\infty}$ form $\alpha$ on $X$ such that $g-g^{\prime} \equiv \alpha$ (cf. [G-S2], Theorem 1.2.2 (i)). The second assertion follows from [G-S2], Corollary 2.2.11. The third follows from the second applied to $X^{\prime} \times X, p r_{2}^{*} Z, p r_{2}^{*} g_{Z}$, and the graph of $f$ in place of $X, Z, g_{Z}$, and $Z^{\prime}$ respectively.

The main properties of the star product on classes of Green currents may be summarized as follows:

Theorem 1.3.2. (i) (Commutativity) Let $Z_{1}$ and $Z_{2}$ be two cycles on $X$ which intersect properly. If $g_{Z_{1}}$ and $g_{Z_{2}}$ are Green currents for $Z_{1}$ and $Z_{2}$, then

$$
\widetilde{g}_{Z_{1}} * \widetilde{g}_{Z_{2}}=\widetilde{g}_{Z_{2}} * \widetilde{g}_{Z_{1}} \text { in } \widetilde{\mathscr{D}}(X) \text {. }
$$

(ii) (Associativity) Let $Z_{1}, Z_{2}, Z_{3}$ be three cycles on $X$ which intersect properly in the following sense: if $p_{i}$ denotes the codimension of $Z_{i}$, we have

$$
\operatorname{codim}_{X}\left(\left|Z_{i}\right| \cap\left|Z_{j}\right|\right)=p_{i}+p_{j} \quad \text { for } i \neq j
$$

and

$$
\operatorname{codim}_{X}\left(\left|Z_{1}\right| \cap\left|Z_{2}\right| \cap\left|Z_{3}\right|\right)=p_{1}+p_{2}+p_{3} .
$$

If $g_{Z_{1}}, g_{Z_{2}}, g_{Z_{3}}$ are Green currents for $Z_{1}, Z_{2}, Z_{3}$, then

$$
\widetilde{g}_{Z_{1}} *\left(\widetilde{g}_{Z_{2}} * \widetilde{g}_{Z_{3}}\right)=\left(\widetilde{g}_{Z_{1}} * \widetilde{g}_{Z_{2}}\right) * \widetilde{g}_{Z_{3}} \text { in } \widetilde{\mathscr{D}}(X) \text {. }
$$

(iii) (Compatibility of star product and pull-back) Let $f: X^{\prime} \rightarrow X$ be a morphism of smooth quasi-projective complex varieties, and let $Z_{1} \in Z^{p_{1}}(X)$ and $Z_{2} \in Z^{p_{2}}(X)$ be cycles on $X$, which intersect properly on $X$, such that $f^{-1}\left(\left|Z_{1}\right|\right), f^{-1}\left(\left|Z_{2}\right|\right)$, and $f^{-1}\left(\left|Z_{1}\right| \cap\left|Z_{2}\right|\right)$ have codimensions $p_{1}, p_{2}$, and $p_{1}+p_{2}$ respectively. If $g_{Z_{1}}$ and $g_{Z_{2}}$ are Green currents for $Z_{1}$ and $Z_{2}$, then

$$
f^{*}\left(\widetilde{g}_{Z_{1}} * \widetilde{g}_{Z_{2}}\right)=f^{*} \tilde{g}_{Z_{1}} * f^{*} \widetilde{g}_{Z_{2}}
$$

and

$$
f^{*}\left(\widetilde{g}_{Z_{1}} \delta_{Z_{2}}\right)=f^{*} \widetilde{g}_{Z_{1}} \delta_{f^{*}\left(Z_{2}\right)}
$$

Proof. The commutativity is Corollary 2.2.9 in [G-S2]. Identity (1.3.4) follows from the associativity by working on $X^{\prime} \times X$ and considering $X^{\prime} \times Z_{1}, X^{\prime} \times Z_{2}$, and the graph of $f$ as in [G-S2], 4.4.3, Lemma. Identity (1.3.5) follows easily 
from (1.3.4), the definition of $f^{*} \widetilde{g}_{Z_{2}}$, and (1.1.1). Associativity is proved in [G-S2], 2.2.14, when $X$ is projective. The following shorter argument avoids this extra hypothesis: let $\omega_{i}=d d^{c} g_{Z_{i}}+\delta_{Z_{i}}$; these are $\partial$ - and $\bar{\partial}$-closed forms, and therefore they act upon $\widetilde{\mathscr{D}}(X)$ by multiplication. By commutativity of the star product, we get:

$$
\begin{aligned}
\widetilde{g}_{Z_{1}} *\left(\widetilde{g}_{Z_{2}} * \widetilde{g}_{Z_{3}}\right) & =\widetilde{g}_{Z_{1}} *\left(\widetilde{g}_{Z_{3}} * \widetilde{g}_{Z_{2}}\right) \\
& =\widetilde{g}_{Z_{1}} \delta_{Z_{3} \cdot Z_{2}}+\omega_{1}\left(\widetilde{g}_{Z_{3}} * \widetilde{g}_{Z_{2}}\right) \\
& =\widetilde{g}_{Z_{1}} \cdot \delta_{Z_{3} \cdot Z_{2}}+\omega_{1} \cdot \widetilde{g}_{Z_{3}} \delta_{Z_{2}}+\omega_{1} \omega_{3} \widetilde{g}_{Z_{2}}
\end{aligned}
$$

On the other hand, using again commutativity, we have

$$
\begin{aligned}
\left(\widetilde{g}_{Z_{1}} * \widetilde{g}_{Z_{2}}\right) * \widetilde{g}_{Z_{3}} & =\widetilde{g}_{Z_{3}} *\left(\widetilde{g}_{Z_{1}} * \widetilde{g}_{Z_{2}}\right) \\
& =\widetilde{g}_{Z_{3}} \cdot \delta_{Z_{1} \cdot Z_{2}}+\omega_{3} \cdot \widetilde{g}_{Z_{1}} \delta_{Z_{2}}+\omega_{3} \omega_{1} \widetilde{g}_{Z_{2}}
\end{aligned}
$$

The equality of (1.3.6) and (1.3.7) follows from [G-S2], Theorem 2.2.2, applied to $Y=Z_{1}, g_{Y}=g_{Z_{1}}, Z=Z_{3}, g_{Z}=g_{Z_{3}}$, and $W=Z_{2}$ (hence $\sigma=\delta_{Z_{2} \cdot Z_{3}}$ and $\tau=0$ ).

Remark. Let us go back to the notation of Proposition 1.3.1. If $\eta$ is any Green form for $Z$ of log type along $|Z|$ such that $g_{Z} \equiv \eta$, the class in $\widetilde{\mathscr{D}}(X)$ (resp. in $\widetilde{\mathscr{D}}\left(X^{\prime}\right)$ ) of $\eta \delta_{Z^{\prime}}$ and $\eta * g_{Z^{\prime}}$ (resp. of $f^{*} \eta$ ) coincide with $\widetilde{g}_{Z} \cdot \delta_{Z^{\prime}}$ and $\widetilde{g}_{Z} * \widetilde{g}_{Z^{\prime}}$ (resp. of $f^{*} \widetilde{g}_{Z}$ ). This follows from the same argument as for Proposition 1.3.1, once we observe that Theorem 2.2.2 in [G-S2] and its corollaries still hold when the cycles $Y, Z$, and $W$ are not supposed irreducible (with the notations of [loc.cit.], one needs only to assume that $|Y| \cap|Z|,|Y| \cap|W|$, and $|Y| \cap|Z| \cap|W|$ have codimensions $p+q, p+r$, and $p+q+r$ respectively, and that $g_{Y}$ and $g_{Z}$ are Green forms for $Y$ and $Z$ of $\log$ type along $|Y|$ and $|Z|$; with trivial modifications, the proof in 2.2.4-2.2.8 still applies under these hypotheses).

Using this more flexible definition, one gets that if

$$
f: X^{\prime} \rightarrow X \text { and } f^{\prime}: X^{\prime \prime} \rightarrow X^{\prime}
$$

are morphisms of smooth quasi-projective complex varieties such that $f^{-1}(|Z|)$ and $\left(f \circ f^{\prime}\right)^{-1}(|Z|)$ have codimension $p$ in $X^{\prime}$ and $X^{\prime \prime}$ respectively, then

$$
f^{\prime *} f^{*} \tilde{g}_{Z}=\left(f \circ f^{\prime}\right)^{*} \tilde{g}_{Z}
$$

1.4. An application: Levine forms and comparison of norms on polynomials. As an illustration of the constructions presented above, we shall use the Levine forms (1.2.3, Example (v)) to compare several notions of size for homogeneous polynomials.

1.4.1. Let $V$ be a complex vector space of dimension $N+1$ equipped with an hermitian scalar product. Then to any linear subspace $W \subset V$ of codimension $p>0$ is associated the Levine form $\Lambda_{\mathbb{P}(W)}$, which is a Green form on $\mathbb{P}(V)$ 
for the subspace $\mathbb{P}(W)$ (see 1.2.3, Example (v)). Its main properties may be summarized as follows:

Proposition 1.4.1. (i) The Levine form $\Lambda_{\mathbf{P}(W)}$ is a positive Green form for $\mathbb{P}(W)$ of log type along $\mathbb{P}(W)$.

(ii) If $\mu$ denotes the Fubini-Study $(1,1)$ form on $\mathbb{P}(V)$ defined by the hermitian structure on $V,{ }^{2}$ the following equation of currents holds:

$$
d d^{c} \Lambda_{\mathbf{P}(W)}+\delta_{\mathbb{P}(W)}=\mu^{p} .
$$

(iii) When $\mathbb{P}(V)$ is equipped with the Kähler structure defined by $\mu$, the harmonic projection of $\Lambda_{\mathbb{P}(W)}$ is given by

$$
H\left(\Lambda_{\mathbf{P}(W)}\right)=\sum_{n=1}^{p} \sum_{m=0}^{N-p} \frac{1}{m+n} \mu^{p-1}
$$

In other words

$$
\int_{\mathbf{P}(V)} \Lambda_{\mathbf{P}(W)} \mu^{N-p+1}=\sum_{n=1}^{p} \sum_{m=0}^{N-p} \frac{1}{m+n} .
$$

Assertions (i) and (ii) have been proved in 1.2.3, Example (v), except the positivity, which follows from the expression (1.2.13) and Proposition 1.1.4, (iii). For a proof of (iii), see [G-S3], Propositions 5.1 and 5.4. Assertions (i) and (ii) go back to Levine ([Lev]), while formula (1.4.1) was first shown by Stoll [St2]. It may also be written as follows. Let

$$
\sigma_{p}=\frac{1}{2} \sum_{k=1}^{p} \sum_{m=1}^{k} \frac{1}{m}=\frac{(p+1)}{2} \sum_{m=1}^{p} \frac{1}{m}-\frac{p}{2} \quad \text { if } p \geq 1,
$$

and $\sigma_{p}=0$ if $p \leq 0$. Then a simple computation using (1.4.1) shows that

$$
H([\Lambda])=2\left(\sigma_{N}-\sigma_{p-1}-\sigma_{N-p}\right) \mu^{p-1} .
$$

1.4.2. Suppose now, to make notation simpler, that $V$ is $\mathbb{C}^{N+1}$ equipped with the standard metric \| $\|$ such that

$$
\left\|\left(z_{0}, \ldots, z_{N}\right)\right\|^{2}=\sum_{i=0}^{N}\left|z_{i}\right|^{2} .
$$

Let $s$ be a regular section of the line bundle $\mathscr{O}(d)$ on $\mathbb{P}^{N}(\mathbb{C})=\mathbb{P}(V)$, i.e., a nonzero homogeneous polynomial $P\left(z_{0}, \ldots, z_{N}\right)$. The standard metric on $\mathbb{C}^{N+1}$ defines a metric on $\mathscr{O}(-1)$ (as in 1.2.3, Example $(\mathrm{v})$ ), hence on its tensor powers. When $\mathscr{O}(d)$ is equipped with this metric, the section $s$ has norm

\footnotetext{
${ }^{2}$ I.e., $\mu$ is the first Chern form of $\mathscr{O}_{V}(1)$ equipped with the metric defined by the scalar product on $V$, cf. 1.2.3, Example (v).
} 


$$
\|s(x)\|=\left(\sum_{i=0}^{N}\left|z_{i}\right|^{2}\right)^{-d / 2}\left|P\left(z_{0}, \ldots, z_{N}\right)\right|
$$

at the point $x \in \mathbb{P}^{N}(\mathbb{C})$ of homogeneous coordinates $\left(z_{0}, \ldots, z_{N}\right)$. Let

$$
\|s\|_{\infty}=\sup _{x \in \mathbb{P}^{N}(\mathbb{C})}\|s(x)\|
$$

and denote by $\mu$ the Fubini-Study $(1,1)$ form on $\mathbb{P}^{N}(\mathbb{C})$ attached to the standard scalar product on $\mathbb{C}^{N+1}$.

Proposition 1.4.2. For any nonzero regular section $s$ of $\mathscr{O}(d)$ over $\mathbb{P}^{N}(\mathbb{C})$, we have:

$$
\log \|s\|_{\infty} \leq \int_{\mathbb{P}^{N}(\mathbb{C})} \log \|s\| \mu^{N}+\frac{d}{2} \sum_{m=1}^{N} \frac{1}{m} .
$$

(Compare with [Fa2], Lemma 2.9; see also [P1], [P2], and [Le2] for related results involving the Mahler measure of polynomials.)

Proof. Let $D=\operatorname{div}(s)$ be the divisor of $s, P \in \mathbb{P}^{N}(\mathbb{C})$ a point outside $D$, and $\Lambda_{P}$ the Levine form of $P$ in $\mathbb{P}^{N}(\mathbb{C})$. Consider the Green current

$$
g_{D}=-\log \|s\|^{2}
$$

for $D$. We have

$$
\omega_{D}:=d d^{c} g_{D}+\delta_{D}=c_{1}(\overline{\mathscr{O}(d)})=d \mu
$$

From the relation

$$
g_{D} * \Lambda_{P} \equiv \Lambda_{P} * g_{D}
$$

(cf. Theorem 1.3.2, (i)) integrated on $\mathbb{P}^{N}(\mathbb{C})$, or more directly from Stokes formula, we get:

$$
g_{D}(P)+d \int_{\mathbb{P}^{N}(\mathbb{C})} \mu \Lambda_{P}=\int_{\mathbb{P}^{N}(\mathbb{C})} \Lambda_{P} \delta_{D}+\int_{\mathbb{P}^{N}(\mathbb{C})} \mu^{N} g_{D}
$$

Using (1.4.2) with $p=N$, the positivity of $\Lambda_{P}$, and (1.1.3), this implies:

$$
-\log \|s\|^{2}(P)+d \sum_{m=1}^{N} \frac{1}{m} \geq-\int_{\mathbb{P}^{N}(\mathbb{C})} \log \|s\|^{2} \mu^{N} .
$$

1.4.3. Remarks. (i) Let $\mathbb{S}^{2 N+1}$ be the unit sphere in $\mathbb{C}^{N+1}$, defined by

$$
\sum_{i=0}^{N}\left|z_{i}\right|^{2}=1
$$


and let $d v$ be the unique $U(N+1)$-invariant probability measure on $\mathbb{S}^{2 N+1}$. The two sides of (1.4.5) may be expressed more concretely in terms of $P$, namely:

$$
\|S\|_{\infty}=\sup _{z \in \mathbb{S}^{2 N+1}}|P(z)|
$$

and

$$
\int_{\mathbb{P}^{N}(\mathbb{C})} \log \|s\| \mu^{N}=\int_{\mathbb{S}^{2 N+1}} \log |P| d v .
$$

(ii) As any hermitian vector space $V$ of dimension $N+1$ is isomorphic to $\mathbb{C}^{N+1}$ equipped with the standard metric, Proposition 1.4.2 immediately extends to the situation where $P^{N}(\mathbb{C})$ is replaced by $\mathbb{P}(V)$, etc.

More generally, let $V_{1}, \ldots, V_{k}$ be hermitian vector spaces of dimensions $N_{1}+1, \ldots, N_{k+1}$, and let

$$
X=\mathbb{P}\left(V_{1}\right) \times \cdots \times \mathbb{P}\left(V_{k}\right) .
$$

The line bundles $\mathscr{O}\left(d_{1}, \ldots, d_{k}\right)$ on $X, d_{i} \in \mathbb{Z}$, are canonically endowed with hermitian metrics (deduced by tensor products and pull back from the metrics on the line bundles $\mathscr{O}_{V_{i}}(-1)$ defined by their injection in the trivial bundle with fiber $V_{i}$ on $\mathbb{P}\left(V_{i}\right)$ ), and there exists a unique probability measure $d v$ on $X$ invariant under the action of $U\left(V_{i}\right) \times \cdots \times U\left(V_{k}\right)$. Define, for any regular section $s$ of $\mathscr{O}\left(d_{1}, \cdots, d_{k}\right)$,

$$
\|s\|_{\infty}=\sup _{x \in X}\|s(x)\|
$$

$$
\|s\|_{0}=\exp \left(\int_{X} \log \|s(x)\| d v(x)\right),
$$

and, for any positive real number $p$,

$$
\|s\|_{p}=\left(\int_{X}\|s(x)\|^{p} d v(x)\right)^{1 / p} .
$$

We know from standard facts on probability spaces that

$$
\|s\|_{\infty} \geq\|s\|_{p} \geq\|s\|_{0}=\lim _{p \rightarrow 0}\|s\|_{p}
$$

(beware that in general \|\|$_{p}$ is not a norm if $p<1$ ). By induction on $k$, we get from Proposition 1.4.2:

Corollary 1.4.3. For any $\left(d_{1}, \ldots, d_{k}\right) \in \mathbb{N}^{k}$ and any regular section $s$ of $\mathcal{O}\left(d_{1}, \ldots, d_{k}\right)$, the following inequality holds:

$$
\|s\|_{\infty} \leq \exp \left(\sum_{i=1}^{k} \sum_{m=1}^{N_{i}} \frac{d_{i}}{2 m}\right)\|s\|_{0} .
$$


(iii) The inequality (1.4.5) is optimal: it becomes an equality when $P$ is the $d$-th power of a linear form. Indeed, if $P=X_{0}^{d}$, then the sup norm (1.4.6) is 1 , while the integral (1.4.7) is $d$ times the integral

$$
\int_{\mathbb{S}^{2 N+1}} \log \left|X_{0}\right| d v_{N}
$$

which is easily shown to be $-\frac{1}{2} \sum_{m=1}^{N} \frac{1}{m}$.

(iv) Let us go back to the notation of 1.4.1. From the proof of Proposition 1.4.2 and the preceding remark, we obtain the following extremal property of Levine forms in the case $p=1:$ the infimum of the integrals

$$
\int_{\mathbb{P}^{N}(\mathbb{C})} g \mu^{N-p+1},
$$

where $g$ runs over the positive Green forms for $\mathbb{P}(W)$ in $\mathbb{P}(V)$ of log type along $\mathbb{P}(W)$ such that

$$
d d^{c} g+\delta_{\mathbb{P}(\boldsymbol{W})}=\mu^{p},
$$

is $2\left(\sigma_{N}-\sigma_{p-1}-\sigma_{N-p}\right)$ and is attained when $g=\Lambda_{\mathbb{P}(W)}$. The general case of this assertion will be proved in 5.1, Remarks, (iii).

1.5. The continuity of some fiber integrals. In this section, we establish the continuity of some integrals associated to families of cycles, which will be used to derive the "basic inequality" in 5.1. The proof will provide another application of the formalism of Green forms of log type.

1.5.1. Let $M$ be a smooth projective complex variety of dimension $d$, and let $T$ be a smooth quasi-projective curve. Let $p: M \times T \rightarrow T$ be the projection onto the second factor, and for any (closed) point $t$ in $T$, let

$$
\begin{aligned}
i_{t}: M & \rightarrow M \times T \\
x & \mapsto(x, t) .
\end{aligned}
$$

If $Z \in Z^{q}(M \times T)$ is a cycle which meets properly every fiber $M \times\{t\}$ of $p$, then, for any $t \in T$, the cycle $i_{t}^{*} Z$ is well defined (in $Z^{q}(M)$ ) and will be denoted $Z_{t}$. Moreover, if $g$ is a Green form for $Z$ of $\log$ type along $|Z|$, then $i_{t}^{*} g$ is a well-defined Green form of log type for $Z_{t}$, which we shall denote $g_{t}$. Similarly, if $\alpha$ is a continuous differential form on $M \times T$, the differential form $i_{t}^{*} \alpha$ on $M$ will be denoted $\alpha_{t}$.

Proposition 1.5.1. Let $Z_{1}$ and $Z_{2}$ be two cycles on $M \times T$, of respective codimensions $p_{1}$ and $p_{2}, p_{1}>0$. Let $g$ be a Green form for $Z_{1}$ of log type along $\left|Z_{1}\right|$ and let $\alpha \in A^{k, k}(M \times T)$ be a closed form, $k=d+1-p_{1}-p_{2}$. Suppose that $Z_{1}$ and $Z_{2}$ meet properly and that, for any $t \in T, Z_{1}, Z_{2}$, and $\left|Z_{1}\right| \cap\left|Z_{2}\right|$ meet $M \times\{t\}$ properly, and consider the current $\alpha . g . \delta_{Z_{2}}$ on $M \times T$ and the currents $\alpha_{t} \cdot g_{t} \cdot \delta_{Z_{2, t}}$ on $M$ (these are well defined according to 1.1.2, since $Z_{1}$ and $Z_{2}$ meet properly, as well as $\left|Z_{1}\right|_{t}$ and $\left.\left|Z_{2}\right|_{t}\right)$. Then the integral 


$$
\varphi(t)=\int_{M} \alpha_{t} \cdot g_{t} \cdot \delta_{Z_{2, t}}
$$

depends continuously on $t \in T$, and the distribution $[\varphi]$ on $T$ coincides with the direct image current $p_{*}\left(\alpha . g . \delta_{Z_{2}}\right)$.

A related result was proved by Stoll [St1], in the case $p_{1}=1$. Applied to $\alpha=1$ and $Z_{1}=0$, Proposition 1.5.1 becomes the following classical result of Federer, Stoll, and King (see [K], 3.3 and 4.1, for a more general statement and references to earlier authors):

Corollary 1.5.2. Let $Z \in Z^{q}(M \times T)$ be a cycle which meets properly the fibers of $p$. For any $\omega \in A^{d-q, d-q}(M \times T)$, the integral

$$
\varphi(t)=\int_{M} \omega_{t} . \delta_{Z_{t}}
$$

depends continuously on $t \in T$. Moreover, we have the equality of currents

$$
[\varphi]=p_{*}\left(\omega . \delta_{Z}\right)
$$

The end of this section is devoted to the proof of Proposition 1.5.1.

1.5.2. Let us begin by proving Proposition 1.5.1 when $Z_{2}=M \times T$. Then it amounts to proving that the integral $\varphi(t)=\int_{M} \alpha_{t} \cdot g_{t}$ depends continuously on $t \in T$. Indeed, in that case the equality of $p_{*}(\alpha . g)$ with the distribution $[\varphi]$ follows from the definition of the direct image of a current and from Fubini's theorem. To simplify notations, we shall write $Z$ instead of $Z_{1}$.

Also observe that it is enough to prove the continuity of $\varphi$ for some Green form $g$ for $Z$ of $\log$ type along $|Z|$. Indeed, if $g^{\prime}$ is another such Green form, there exists $u \in A^{p_{1}-1, p_{1}-1}(M \times T)$ such that

$$
g^{\prime}-g-u \in \partial \mathscr{D}^{p_{1}-2, p_{1}-1}(M \times T)+\bar{\partial} \mathscr{D}^{p_{1}-1, p_{1}-2}(M \times T)
$$

([G-S2], Theorem 1.2.2, (i)). Then according to Proposition 1.3.1, (iii) for any $t \in T$ we have:

$$
g_{t}^{\prime}-g_{t}-u_{t} \in \partial \mathscr{D}^{p_{1}-2, p_{1}-1}(M)+\bar{\partial} \mathscr{D}^{p_{1}-1, p_{1}-2} J(M) .
$$

Since $\alpha$ is a $\partial$ - and $\bar{\partial}$-closed form this implies, by Stokes formula,

$$
\int_{M} \alpha_{t} \cdot g_{t}^{\prime}=\int_{M} \alpha_{t} \cdot g_{t}+\int_{M} \alpha_{t} \cdot u_{t},
$$

and the last integral defines a continuous (indeed $C^{\infty}$ ) function of $t \in T$.

Let $\bar{T}$ be the smooth projective compactification of $T$, and $\bar{Z}$ the closure of $Z$ in $M \times \bar{T}$. We shall prove the continuity of $\int_{M} \alpha_{t} \cdot g_{t}$ when $g$ is (the restriction to $M \times T$ of) a Green form for $\bar{Z}$ in $M \times \bar{T}$, of log type along $|\bar{Z}|$.

We shall use the following notation: let

$$
p: M \times \bar{T} \rightarrow \bar{T} \text { and } q: M \times \bar{T} \rightarrow M
$$


be the two projections, let

$$
\omega=d d^{c} g+\delta_{Z}\left(\in A^{p_{1} p_{1}}(M \times \bar{T})\right),
$$

and choose $h \in \mathscr{D}^{00}(\bar{T} \times \bar{T})$ a Green current for the diagonal $\Delta_{\bar{T}}$ in $\bar{T} \times \bar{T}$. Such a Green current is indeed a Green form of log type along the diagonal; namely, if $z$ is a local holomorphic coordinate, we can write locally

$$
h\left(t_{1}, t_{2}\right)=\log \left|z\left(t_{1}\right)-z\left(t_{2}\right)\right|^{-2}+\varphi\left(t_{1}, t_{2}\right),
$$

where $\varphi$ is smooth. For any $t \in \bar{T}$ the function

$$
h_{t}=h(t, .)
$$

is a Green form for the point $t$ in $\bar{T}$, and we let

$$
\mu_{t}=d d^{c} h_{t}+\delta_{t} \in A^{1,1}(\bar{T}) .
$$

For any $t \in T$ we have, by definition of $g_{t}$ and $g . \delta_{M \times\{t\}}$ :

$$
\int_{M} \alpha_{t} \cdot g_{t}=\int_{M \times \bar{T}} q^{*} \alpha_{t} \cdot g \cdot \delta_{M \times\{t\}}
$$

On the other hand:

$$
g . \delta_{M \times\{t\}}=g \cdot p^{*} \delta_{\{t\}}=g * p^{*} h_{t}-\omega \cdot p^{*} h_{t}
$$

and, according to Theorem 1.3.2, (i):

$$
g * p^{*} h_{t} \equiv p^{*} h_{t} * g \equiv p^{*} h_{t} \cdot \delta_{Z}+p^{*} \mu_{t} \cdot g \text {. }
$$

From (1.5.2), (1.5.3), (1.5.4), and the fact that $\alpha_{t}$ is $\partial$ - and $\bar{\partial}$-closed, we get:

$$
\int_{M} \alpha_{t} \cdot g_{t}=\int_{M \times \bar{T}} q^{*} \alpha_{t} \cdot p^{*} h_{t} \cdot \delta_{Z}+\int_{M \times \bar{T}} q^{*} \alpha_{t} \cdot p^{*} \mu_{t} \cdot g-\int_{M \times \bar{T}} q^{*} \alpha_{t} \cdot \omega \cdot p^{*} h_{t}
$$

The last two integrals on the right-hand side of (1.5.5) are easily seen to be $C^{\infty}$ functions of $t \in T$. To prove the continuity of the first one, we may clearly suppose that $Z$ is irreducible. Let then $\nu: \widetilde{Z} \rightarrow Z \subset M \times T$ be a resolution of $Z$. According to (1.1.2), we get

$$
\int_{M \times \bar{T}} q^{*} \alpha_{t} \cdot p^{*} h_{t} \cdot \delta_{Z}=\int_{\widetilde{Z}}(q \circ \nu)^{*} \alpha_{t} \cdot(p \circ \nu)^{*} h_{t} .
$$

In terms of local holomorphic coordinates $x_{1}, \ldots, x_{N}$ on $\widetilde{Z}$ and $z$ on $T$, the differential form under the sign $\int_{\widetilde{Z}}$ may be written as

$$
\begin{aligned}
& a\left(x_{1}, \ldots, x_{N}, z(t)\right) \\
& \quad \times\left[\log \left|f\left(x_{1}, \ldots, x_{N}\right)-z(t)\right|+\rho\left(f\left(x_{1}, \ldots, x_{N}\right), z(t)\right)\right] \prod_{i=1}^{N} d x_{i} \wedge d \bar{x}_{i},
\end{aligned}
$$


for some $C^{\infty}$ functions $a$ and $\rho$ and some nonconstant holomorphic function $f$ (use (1.5.1)). According to the Weierstrass preparation theorem, after a possible linear change in the local coordinates $\left(x_{1}, \ldots, x_{N}\right)$, we may write, for $x_{1}, \ldots, x_{N}$ and $z$ in some neighborhood of 0 :

$$
\begin{aligned}
& f\left(x_{1}, \ldots, x_{N}\right)-z \\
& \quad=\left(x_{1}^{d}+\sum_{j=1}^{d} b_{j}\left(x_{2}, \ldots, x_{N}, z\right) x_{1}^{d-j}\right) q\left(x_{1}, \ldots, x_{N}, z\right),
\end{aligned}
$$

where $d \in \mathbb{N}$, and $b_{1}, \ldots, b_{d}$ and $q$ are holomorphic functions such that $b_{1}(0, \ldots, 0)=\cdots=b_{d}(0, \ldots, 0)=0$ and $q(0, \ldots, 0) \neq 0$. Using partitions of unity, this shows that the continuity of (1.5.6) is a consequence of the second assertion of the following

Lemma 1.5.3. Let $U \subset \mathbb{C}, V \subset \mathbb{C}^{N-1}$, and $W \subset \mathbb{C}$ be open subsets. Let $b_{1}, \ldots, b_{d}$ be holomorphic functions on $V \times W$ and $\rho$ a compactly supported continuous function on $U \times V \times W$.

(i) The integral

$$
\int_{U} \rho\left(x_{1}, \ldots, x_{N}, z\right) \log \left|x_{1}^{d}+\sum_{j=1}^{d} b_{j}\left(x_{2}, \ldots, x_{N}, z\right) x_{1}^{d-j}\right| d x_{1} \wedge d \bar{x}_{1}
$$

defines a continuous function $\psi$ of $\left(x_{2}, \ldots, x_{N}, z\right) \in V \times W$.

(ii) The integral

$$
\int_{U \times V} \rho\left(x_{1}, \ldots, x_{N}, z\right) \log \left|x_{1}^{d}+\sum_{j=1}^{d} b_{j}\left(x_{2}, \ldots, x_{N}, z\right) x_{1}^{d-j}\right| \prod_{i=1}^{N} d x_{i} \wedge d \bar{x}_{i}
$$

defines a continuous function $\varphi$ of $z \in W$.

Proof of Lemma 1.5.3. (i) If $\lambda_{1}, \ldots, \lambda_{d}$ denote the roots of the polynomial

$$
X^{d}+\sum_{j=1}^{d} b_{j}\left(x_{2}, \ldots, x_{N}, z\right) X^{d-j},
$$

the integral (1.5.7) may be written

$$
\int_{U} \rho\left(x_{1}, \ldots, x_{N}, z\right) \log \left|\prod_{j=1}^{d}\left(x_{1}-\lambda_{j}\right)\right| d x_{1} \wedge d \bar{x}_{1} .
$$

This is clearly equal to

$$
\sum_{j=1}^{d} \int_{\mathbb{C}} \rho\left(u+\lambda_{j}, x_{2}, \ldots, x_{N}, z\right) \log |u| d u \wedge \overline{d u} .
$$


This expression depends continuously on $\left(\lambda_{1}, \ldots, \lambda_{d}, x_{2}, \ldots, x_{N}, z\right)$. Therefore the continuity of $(1.5 .7)$ as a function of $\left(x_{2}, \ldots, x_{N}, z\right)$ follows from the continuity of the roots of a polynomial.

(ii) By Fubini's theorem, we have

$$
\varphi(z)=\int_{V} \psi\left(x_{2}, \ldots, x_{N}, z\right) \prod_{j=2}^{N} d x_{j} \wedge d \bar{x}_{j} .
$$

The function $\rho$ is compactly supported, and so is $\psi$. Therefore the continuity of $\varphi$ follows from (1.5.9) and the continuity of $\psi$.

1.5.3. Let us finally prove Proposition 1.5 .1 in complete generality. Let $g_{2}$ be a Green form of log type for $Z_{2}$. Then $g * g_{2}$ is a Green current for the intersection cycle $I=Z_{1} . Z_{2}$, and there is a Green form of log type $h$ such that

$$
h \equiv g * g_{2} \text {. }
$$

Let $\omega:=d d^{c} g+\delta_{Z_{1}}$. It is a smooth closed form of type $\left(p_{1}, p_{1}\right)$, and we have

$$
g . \delta_{Z_{2}}=g * g_{2}-\omega g_{2} \equiv h-\omega g_{2}
$$

This implies

$$
p_{*}\left(\alpha . g . \delta_{Z_{2}}\right)=p_{*}(\alpha . h)-p_{*}\left(\alpha \cdot \omega \cdot g_{2}\right)
$$

indeed, as $\alpha$ is $\partial$ - and $\bar{\partial}$-closed, (1.5.10) implies that the two sides of this equality differ by a current of the form $p_{*} \partial u+p_{*} \bar{\partial} v$; since $p_{*}$ commutes with $\partial$ and $\bar{\partial}$, they must be equal in $\widetilde{\mathscr{D}}(T)$, hence in $\mathscr{D}(T)$, since they are currents of degree zero. On the other hand, according to Proposition 1.3.1, (ii), Theorem 1.3.2, (iii) and 1.3, Remark, we know that, for any $t \in T$ :

$$
g_{t} \cdot \delta_{Z_{2}} \equiv h_{t}-\omega_{t} \cdot g_{2, t}
$$

Since $\alpha_{t}$ is $\partial$ - and $\bar{\partial}$-closed, it follows that

$$
\int_{M} \alpha_{t} \cdot g_{t} \cdot \delta_{Z_{2, t}}=\int_{M} \alpha_{t} \cdot h_{t}-\int_{M} \alpha_{t} \cdot \omega_{t} \cdot g_{2, t} .
$$

Taken together, (1.5.11) and (1.5.12) show that to prove Proposition 1.5.1, it is enough to prove it with $\left(Z_{1}, Z_{2}, \alpha, g\right)$ replaced by $(I, M \times T, \alpha, h)$ or by $\left(Z_{2}, M \times T, \alpha \omega, g_{2}\right)$. As we already established Proposition 1.5.1 when $Z_{2}=M \times T$, this completes the proof. 


\section{Preliminaries: Arithmetic intersection theory}

\subsection{Arithmetic Chow groups and Chern classes.}

2.1.1. Arithmetic Chow groups. Let $K$ be a number field of degree $[K: \mathbb{Q}]$, $\mathscr{O}_{K}$ its ring of integers, and $S=\operatorname{Spec}\left(\mathscr{O}_{K}\right)$ the associated scheme. For any imbedding $\sigma: K \rightarrow \mathbb{C}$ and any $K$-scheme or $S$-scheme $X$, we shall denote by $X_{\sigma}$ the $\mathbb{C}$-scheme deduced from $X$ by the base change $\sigma: \operatorname{Spec}(\mathbb{C}) \rightarrow \operatorname{Spec}(K)$ $(\in S)$. Similarly, if $f: X \rightarrow Y$ is a morphism of $K$-schemes we shall denote by $f_{\sigma}: X_{\sigma} \rightarrow Y_{\sigma}$ the morphism of $\mathbb{C}$-schemes it induces by base change. These notations will be used throughout this paper.

An arithmetic variety is, by definition, a scheme $X$ which is flat and quasiprojective over $S$ and whose generic fiber $X_{K}=X \times_{S} \operatorname{Spec}(K)$ is regular. A projective arithmetic variety is an arithmetic variety which is projective over $S$. Observe that any (projective) arithmetic variety over $S$ may be seen as a (projective) arithmetic variety over $\operatorname{Spec}(\mathbb{Z})$. A morphism between two arithmetic varieties $X$ and $Y$ will be any morphism of schemes from $X$ to $Y$, not necessarily an $S$-morphism.

For any arithmetic variety $X$ and any integer $p \geq 0$ we let $Z_{p}(X)$ (resp. $\left.Z^{p}(X)\right)$ be the group of cycles of dimension $p$ (resp. codimension $p$ ) over $X$, i.e. the free abelian group on the set of points of dimension $p$ (resp. codimension $p$ ) of $X$. For any such cycle $Z$ we denote by $|Z| \subset X$ its support.

The set $X(\mathbb{C})$ of complex points of the scheme $X$ may be identified with the disjoint union $\coprod_{\sigma: K \rightarrow \mathbb{C}} X_{\sigma}(\mathbb{C})$. Let $F_{\infty}: X(\mathbb{C}) \rightarrow X(\mathbb{C})$ be the antiholomorphic involution coming from complex conjugation of the coordinates of complex points in $X$. We denote by $A^{p p}\left(X_{\mathbb{R}}\right)$ (resp. $D^{p p}\left(X_{\mathbb{R}}\right)$ ) the set of real forms $\alpha \in$ $A^{p p}(X(\mathbb{C}))$ (resp. real currents $\left.\alpha \in D^{p p}(X(\mathbb{C}))\right)$ such that $F_{\infty}^{*}(\alpha)=(-1)^{p} \alpha$. The image of $A^{p p}\left(X_{\mathbb{R}}\right)$ (resp. $\left.D^{p p}\left(X_{\mathbb{R}}\right)\right)$ in $\widetilde{A}^{p p}\left(X(\mathbb{C})\right.$ ) (resp. $\widetilde{\mathscr{D}}^{p p}\left(X_{\mathbb{R}}\right)$ ) will be denoted $\widetilde{A}^{p p}\left(X_{\mathrm{R}}\right)$ (resp. $\left.\widetilde{D}^{p p}\left(X_{\mathrm{R}}\right)\right)$.

Any cycle $Z$ in $Z^{p}(X)$ defines a current $\delta_{Z} \in D^{p p}\left(X_{\mathbb{R}}\right)$ by integration on its set of complex points: if $Z=\sum_{\alpha} n_{\alpha} Z_{\alpha}, \delta_{Z}=\sum_{\alpha} n_{\alpha} \delta_{Z_{\alpha}(\mathbb{C})}$. A Green current for $Z$ is any current $g \in D^{p-1, p-1}\left(X_{\mathbb{R}}\right)$ such that $d d^{c} g+\delta_{Z}$ is smooth (i.e., a Green current for $Z(\mathbb{C})$, in the sense of 1.1 .1 , which lies in $\left.\mathscr{D}^{p-1, p-1}\left(X_{\mathbb{R}}\right)\right)$.

Let $X$ be a regular arithmetic variety. We let $\widehat{Z}^{p}(X)$ be the group of pairs $(Z, g)$ where $Z \in Z^{p}(X)$ and $g$ is a Green current for $Z$, with addition defined componentwise. Let $\widehat{R}^{p}(X) \subset \widehat{Z}^{p}(X)$ be the subgroup generated by pairs of the form $(0, \partial u+\bar{\partial} v)$ or $\left(\operatorname{div}(f),-\log |f|^{2}\right)$, where $f \in k(Y)^{*}$ is a nontrivial rational function on an integral subscheme $Y \subset X$ of codimension $p-1$, and $-\log |f|^{2}$ is the current on $X(\mathbb{C})$ obtained by restricting forms to the smooth part of $Y(\mathbb{C})$ and integrating against the $L^{1}$ function $-\log |f|^{2}$. The arithmetic Chow group of codimension $p$ of $X$ is

$$
\widehat{C H}^{p}(X)=\widehat{Z}^{p}(X) / \widehat{R}^{p}(X) .
$$


We refer the reader to [G-S2] for more details on this definition, as well as for the properties of this group, some of which we shall now recall (see also [Gi], [B2], and [So-A-B-K] for expository presentations of arithmetic intersection theory).

There exist group morphisms

$$
\begin{gathered}
z: \widehat{C H}^{p}(X) \rightarrow C H^{p}(X), \quad \omega: \widehat{C H}^{p}(X) \rightarrow A^{p p}\left(X_{\mathbb{R}}\right), \\
a: \widetilde{A}^{p-1, p-1}\left(X_{\mathbb{R}}\right) \rightarrow \widehat{C H}^{p}(X)
\end{gathered}
$$

defined as follows: the map $z$ sends the class of $(Z, g)$ to the class of $Z$ in the usual (algebraic) Chow group of $X$; the map $\omega$ sends $(Z, g)$ to $d d^{c} g+\delta_{Z}$ (which is smooth by the definition of Green currents); and $a$ sends the class $\tilde{\eta}$ of $\eta \in A^{p-1, p-1}\left(X_{\mathbf{R}}\right) \subset D^{p-1, p-1}\left(X_{\mathbf{R}}\right)$ to the class of $(0, \eta)$. Notice that $\omega \circ a=d d^{c}$.

The following statement is a consequence of Hodge Theory (cf. [G-S2], 1.2.3 and 3.3 .5$, p.130):

Lemma 2.1.1. Let $X$ be a regular arithmetic variety such that $X_{K}$ is projective. Assume $X(\mathbb{C})$ is endowed with an $F_{\infty}$-invariant Kähler structure. If $x \in \widehat{C H}^{p}(X)$ is such that $z(x)=0$ and $\omega(x)=0$, there exists a harmonic form $\eta$ in $A^{p-1, p-1}\left(X_{\mathbb{R}}\right)$ such that $x=a(\tilde{\eta})$.

Any morphism $f: X \rightarrow Y$ of regular arithmetic varieties induces a group morphism

$$
f^{*}: \widehat{C H}^{p}(Y) \rightarrow \widehat{C H}^{p}(X) .
$$

When $X$ and $Y$ are equidimensional, $f$ is proper, and its restriction to $X_{K}$ is smooth, there exists also a push-forward morphism $f_{*}: \widehat{C H}^{p}(X) \rightarrow \widehat{C H}^{p-\delta}(Y)$, where $\delta=\operatorname{dim}(X)-\operatorname{dim}(Y)$. The push-forward $f_{*}$ is defined by the following formula:

$$
f_{*}[(Z, g)]=\left[\left(f_{*} Z, f_{\mathbb{C} *} g\right)\right],
$$

and the pull-back $f^{*}$ is defined "formally" by

$$
f^{*}[(Z, g)]=\left[\left(f^{*} Z, f_{\mathbb{C}}^{*} g\right)\right],
$$

when $f$ meets $Z$ properly in the generic fiber and $g$ is a Green form of $\log$ type along $|Z|(\mathbb{C})$.

Furthermore, there is a cup-product

$$
\widehat{C H}^{p}(X) \otimes \widehat{C H}^{q}(X) \rightarrow \widehat{C H}^{p+q}(X)_{\mathbb{Q}},
$$

"formally" defined by the formula:

$$
\left[\left(Z_{1}, g_{1}\right)\right]\left[\left(Z_{2}, g_{2}\right)\right]=\left[\left(Z_{1} . Z_{2}, g_{1} * g_{2}\right)\right],
$$

when $Z_{1}$ and $Z_{2}$ meet properly in the generic fiber, and $g_{1}$ and $g_{2}$ are Green forms of log type along $\left|Z_{1}\right|(\mathbb{C})$ and $\left|Z_{2}\right|(\mathbb{C})$ (see [G-S2], 4.1-4.2, and 2.2 below for more details). 
When $X$ is smooth over $S$, the cup-product lies in $\widehat{C H}^{p+q}(X)$ itself ([G-S2], 4.5). Both $z$ and $\omega$ are ring morphisms. Given $f$ as above, one has $f^{*}(x y)=f^{*}(x) f^{*}(y)$, the construction of $f_{*}$ and $f^{*}$ are functorial, and, if $f$ is proper on $X$ and is smooth on its generic fiber, $f_{*}\left(x f^{*}(y)\right)=f_{*}(x) y$ (projection formula). Note also the following useful formula:

$$
a(\eta) y=a(\eta \omega(y))
$$

2.1.2. Arithmetic Chern classes. An hermitian vector bundle on the arithmetic variety $X$ is a pair $\bar{E}=(E, h)$, where $E$ is a locally free coherent $\mathscr{O}_{X}$-module and $h$ is a $C^{\infty}$ hermitian scalar product on the holomorphic vector bundle $E_{\mathbb{C}}$ on $X(\mathbb{C})$; it is also assumed that $h$ is invariant under $F_{\infty}$. If $X$ is regular, for any $p \geq 0$, one can define a Chern class

$$
\widehat{c}_{p}(\bar{E}) \in \widehat{C H}^{p}(X)
$$

(see [G-S3], §4). By definition, $\widehat{c}_{0}(\bar{E})=1$ in $\widehat{C H}^{0}(X)=\mathbb{Z}$ (when $X$ is irreducible) and $\widehat{c}_{1}(\bar{E})=\widehat{c}_{1}(\operatorname{det} \bar{E})$, where $\operatorname{det} \bar{E}$ is the maximal exterior power of $\bar{E}$. If $\bar{L}$ is an hermitian line bundle, $\widehat{c}_{1}(\bar{L})$ is the class of the pair $\left(\operatorname{div}(s),-\log \|s\|^{2}\right)$ for any rational section $s$ of $L$ over $X$ of norm $\|s\|$ on $X(\mathbb{C})$ (so that $-\log \|s\|^{2}$ is a distribution on $X(\mathbb{C})$ ). For any $\bar{E}$ and $p \geq 0$, the form

$$
c_{p}(\bar{E})=\omega\left(\widehat{c}_{p}(\bar{E})\right) \in A^{p p}\left(X_{\mathbb{R}}\right)
$$

is the usual $p$-th Chern form of the hermitian vector bundle $\bar{E}_{\mathbb{C}}$ over $X(\mathbb{C})$. As for usual Chern classes, if $r$ denotes the rank of $E, \widehat{c}_{p}(\bar{E})=0$ if $p>r$.

Given an exact sequence

$$
\mathscr{E}: 0 \rightarrow E^{\prime} \rightarrow E \rightarrow E^{\prime \prime} \rightarrow 0
$$

of vector bundles on $X$ and any choice $h^{\prime}, h, h^{\prime \prime}$ of metrics on $E^{\prime}, E, E^{\prime \prime}$ respectively, the following formula holds in $\widehat{C H}^{p}(X)_{\mathbb{Q}}$ :

$$
\widehat{c}_{p}(\bar{E})=\sum_{r+s=p} \widehat{c}_{r}\left(E^{\prime}\right) \cdot \widehat{c}_{s}\left(E^{\prime \prime}\right)-a\left(\widetilde{c}_{p}(\mathscr{E})\right),
$$

where $\widetilde{c}_{p}(\mathscr{E}) \in \widetilde{A}^{p-1, p-1}\left(X_{\mathbf{R}}\right)$ is the Bott-Chern secondary characteristic class attached to $\left(\mathscr{E}, h^{\prime}, h, h^{\prime \prime}\right)$ [B-C], [G-S3]. It is convenient to introduce the total Chern class

$$
\widehat{c}(\bar{E})=\sum_{p \geq 0} \widehat{c}_{p}(\bar{E}) \in \widehat{C H}^{*}(X)
$$

and the total Bott-Chern secondary class

$$
\widetilde{c}(\mathscr{E})=\sum_{p \geq 0} \widetilde{c}_{p}(\bar{E}) \in \widetilde{A}^{*}(X) .
$$


Then (2.1.3) may be written as

$$
\widehat{c}(\bar{E})=\hat{c}\left(\overline{E_{.}^{\prime}}\right) \cdot \hat{c}\left(\overline{E^{\prime \prime}}\right)-a(\widetilde{c}(\mathscr{E})) .
$$

In particular, if $\overline{E^{\prime}} \oplus \overline{E^{\prime \prime}}$ denotes the direct sum of $E^{\prime}$ and $E^{\prime \prime}$ endowed with the orthogonal sum of their metrics,

$$
\widehat{c}\left(\overline{E^{\prime}} \oplus \overline{E^{\prime \prime}}\right)=\widehat{c}\left(\overline{E^{\prime}}\right) \cdot \widehat{c}\left(\overline{E^{\prime \prime}}\right) \text { in } \widehat{C H}^{*}(X)_{\mathbb{Q}},
$$

where $\widehat{C H}^{*}(X)=\underset{p \geq 0}{\oplus} \widehat{C H}^{p}(X)$. When $X$ is smooth over $S$, (2.1.4) and (2.1.5) hold in $\widehat{C H}^{*}(X)$. See [G-S3] for more details.

These constructions extend to an arbitrary arithmetic variety $X$ (so there might exist singularities on closed fibers) [G-S6]. One then defines arithmetic Chow homology groups $\widehat{C H}_{p}(X)$, generated by pairs $(Z, g)$ where $Z \in Z_{p}(X)$ and $g$ is a Green current for $Z$. These are covariant for proper maps which are smooth on the generic fiber, and contravariant for flat maps with constant relative dimension. Given any map $f: X \rightarrow Y$, where $X$ and $Y$ are as above and $Y$ is regular, there exists a cap-product

$$
\widehat{C H}^{q}(Y) \otimes \widehat{C H}_{p}(X) \stackrel{\cdot}{\rightarrow} \widehat{C H}_{p-q}(X)_{Q}
$$

which generalizes the pairing (2.1.1). An hermitian vector bundle $\bar{E}$ on $X$ defines "operational" Chern classes, i.e., morphisms

$$
\widehat{c}_{q}(\bar{E}) \cap .: \widehat{C H}_{p}(X) \rightarrow \widehat{C H}_{p-q}(X),
$$

for all $p \geq 0$ which is compatible with the cap-product (2.1.6): if $\bar{F}$ is an hermitian vector bundle over $Y$ such that $\bar{E} \simeq f^{*} \bar{F}$, then for any $x \in \widehat{C H}_{p}(X)$, we have

$$
x \cap \widehat{c}_{q}(\bar{E})=x \cdot{ }_{f} \widehat{c}_{q}(\bar{F}) .
$$

We refer to [G-S6], $\S 2.3$, for these notions and their properties.

2.1.3. The degree maps on $\widehat{C H}^{*}\left(\operatorname{Spec}\left(\mathscr{O}_{K}\right)\right)$. In the case of

$$
X=S=\operatorname{Spec}\left(\mathscr{O}_{K}\right) \text {, }
$$

the group $\widehat{C H}^{p}(X)$ vanishes when $p>1$. We shall denote by

and

$$
\operatorname{deg}_{K}: \widehat{C H}^{*}(S) \rightarrow \mathbb{Z}
$$

$$
\widehat{\operatorname{deg}}: \widehat{C H}^{*}(S) \rightarrow \mathbb{R}
$$

the following morphisms. The map $\operatorname{deg}_{K}$ (the algebraic degree map) is just the projection

$$
\widehat{C H}^{*}(S) \rightarrow \widehat{C H}^{0}(S) \stackrel{\sim}{\rightarrow} C H^{0}(S)=\mathbb{Z},
$$


while $\widehat{\mathrm{deg}}$ (the arithmetic degree map) is the composition of the projection $\widehat{C H}^{*}(S) \rightarrow \widehat{C H}^{1}(S)$ of the push-forward morphism

$$
\widehat{C H}^{1}(S) \rightarrow \widehat{C H}^{1}(\operatorname{Spec}(\mathbb{Z}))
$$

attached to the unique morphism $S \rightarrow \operatorname{Spec}(\mathbb{Z})$, and of the isomorphism

$$
\widehat{C H}^{1}(\operatorname{Spec}(\mathbb{Z})) \stackrel{\sim}{\rightarrow} \mathbb{R}
$$

the inverse of which maps the class of $(0,2 \lambda)$ to the real number $\lambda \in \mathbb{R}$.

An element of $\hat{Z}^{1}(S)$ is a couple $\left(\sum_{\wp} n_{\wp} \wp, \lambda\right)$, where $\wp$ runs over the prime ideals in $\mathscr{O}_{K}$, the $n_{\wp}$ are integers, almost all zero, and $\lambda$ is an $F_{\infty}$ invariant function from $S(\mathbb{C})$ to $\mathbb{R}$, i.e., a map $\lambda: \sigma \rightarrow \lambda(\sigma)$ on the set of $[K: \mathbb{Q}]$ imbeddings $\sigma: K \rightarrow \mathbb{C}$ such that $\lambda(\bar{\sigma})=\lambda(\sigma)$. One easily sees that

$$
\widehat{\operatorname{deg}}\left[\left(\sum_{\wp} n_{\wp} \wp, \lambda\right)\right]=\sum_{\wp} n_{\wp} . N \wp+\frac{1}{2} \sum_{\sigma: K \rightarrow \mathbb{C}} \lambda(\sigma),
$$

where $N \wp$ denotes the norm of $\wp$.

It follows from [G-S2], 3.4.1 and 3.4.3, that

$$
\operatorname{deg}_{K}: \widehat{C H}^{0}(S) \rightarrow \mathbb{Z}
$$

is an isomorphism and that the map

$$
\widehat{\operatorname{deg}}: \widehat{C H}^{1}(S) \rightarrow \mathbb{R}
$$

is onto, with kernel a compact group (namely an extension of the ideal class group of $K$ by the compact torus, quotient of $\mathbb{R}^{r_{1}+r_{2}-1}$ by the image of Dirichlet's regulator map).

The multiplicative structure of $\widehat{C H}^{*}(S)$ is just given by its $\left(\mathbb{Z}=\widehat{C H}^{0}(S)\right)$ module structure (the product of two elements in $\widehat{C H}^{1}(S)$ vanishes). It follows that

$$
\operatorname{deg}_{K}(x y)=\operatorname{deg}_{K}(x) \operatorname{deg}_{K}(y)
$$

and

$$
\widehat{\operatorname{deg}}(x y)=\widehat{\operatorname{deg}}(x) \operatorname{deg}_{K}(y)+\operatorname{deg}_{K}(x) \widehat{\operatorname{deg}}(y) .
$$

An hermitian vector bundle $\bar{E}$ on $S$ is the same as the data consisting of a finitely generated projective $\mathscr{O}_{K}$-module $E$, and of hermitian scalar products on the $[K: \mathbb{Q}]$ complex vector spaces $E_{\sigma}$ associated to the imbeddings $\sigma: K \rightarrow \mathbb{C}$, which are invariant under the involution $F_{\infty}$. The real vector space $E \otimes_{\mathbb{Z}} \mathbb{R}$ is then naturally endowed with a euclidean scalar product, namely the restriction of the hermitian scalar product on 


$$
E \otimes_{\mathbb{Z}} \mathbb{C} \simeq \bigoplus_{\sigma: K \rightarrow \mathbb{Q}} E_{\sigma}
$$

defined as the direct sum of the given scalar products on the $E_{\sigma}$ 's.

The arithmetic degree of the hermitian vector bundle $\bar{E}$ on $S$ is defined as the real number

$$
\widehat{\operatorname{deg}}(\bar{E})=\widehat{\operatorname{deg}}\left(\widehat{c}_{1}(\bar{E})\right) .
$$

If $V$ is the covolume of the lattice $E$ in the euclidean vector space $E \otimes_{\mathbb{Z}} \mathbb{R}$, and $\Delta_{K}$ the discriminant of the number field $K$, we have

$$
\widehat{\operatorname{deg}}(\bar{E})=-\log V+\frac{1}{2} \mathrm{rk} E \cdot \log \left|\Delta_{K}\right| \text {. }
$$

Note also that

$$
\widehat{\operatorname{deg}}(\bar{E})=\widehat{\operatorname{deg}}(\operatorname{det} \bar{E})
$$

and, when $\bar{L}$ is an hermitian line bundle over $S$,

$$
\widehat{\operatorname{deg}}(\bar{L})=\log \#\left(L / \mathscr{O}_{K} s\right)-\sum_{\sigma} \log \|s\|_{\sigma},
$$

where \# stands for the cardinality of a finite set, $s$ is any nonzero element in $L$, $\sigma: K \rightarrow \mathbb{C}$ runs over the $[K: \mathbb{Q}]$ imbeddings of $K$ in $\mathbb{C}$, and $\|s\|_{\sigma}$ is the norm of $s$ in $L_{\sigma}$. Formula (2.1.15) is a consequence of (2.1.8) and the definitions of $\widehat{c}_{1}(\bar{L})$; when $E$ is trivial, (2.1.13) follows after a short computation from (2.1.14), (2.1.15) applied to a trivializing section of $\operatorname{det} E$, and the definition of $\Delta_{K}$; one reduces to this case by considering $E^{\oplus h}$, where $h$ is the order of the ideal class group of $K$.

For more details on these notions, we refer to [Sz], [La], and [G-S7].

2.2. The intersection product. Before going on, we shall describe more precisely the intersection pairing (2.1.1) on a regular arithmetic variety $X$ (see [G-S2], 4.2.3).

First recall that if $A$ and $B$ are Zariski closed subsets in $X$, there exists a pairing

$$
C H_{A}^{p}(X) \otimes C H_{B}^{q}(X) \rightarrow C H_{A \cap B}^{p+q}(X)_{\mathbb{Q}}
$$

on Chow groups with supports. This product is associative and compatible with restriction to open subsets in $X$ and with enlargement of supports. It can be defined by means of the isomorphism between $C H_{A}^{p}(X)_{\mathbb{Q}}$ and the weight $p$ part of the $K$-theory with supports $K_{0}^{A}(X)_{\mathbb{Q}}$ [So3], [G-S1]. When $X$ is smooth over $S$, this pairing takes values in $C H_{A \cap B}^{p+q}(X)$ and can be defined as in Fulton [Fu2] (the results in that book extend to that case, see [Fu2], 20.2).

Assume now that $Y \in Z^{p}(X)$ and $Z \in Z^{q}(X)$ are algebraic cycles on $X$, and consider their fundamental classes $[Y] \in C H_{|Y|}^{p}(X)$ and $[Z] \in C H_{|Z|}^{q}(X)$. We may then look at their product 


$$
[Y][Z] \in C H_{|Y| \cap|Z|}^{p+q}(X)_{\mathbb{Q}} .
$$

If $U \subset X$ is an open subset where $Y$ and $Z$ meet properly, the restriction of $[Y][Z]$ to

$$
C H_{|Y| \cap|Z| \cap U}^{p+q}(U)_{\mathbb{Q}}=Z^{p+q}(|Y| \cap|Z| \cap U)_{\mathbb{Q}}
$$

is the usual intersection cycle $\sum_{\alpha} m_{\alpha} W_{\alpha}$, where $W_{\alpha}$ are the irreducible components of $|Y| \cap|Z| \cap U$ and $m_{\alpha}$ are the Serre intersection multiplicities [G-S1].

When $p=1$, the image of $[Y][Z]$ in $C H_{|Z|}^{p+q}(X)_{\mathbb{Q}}$ agrees with the class considered in [Fu2], 2.3. When $Z$ is contained in $|Y|$ this follows from the fact that $\operatorname{Pic}(X)_{\mathbb{Q}}$ is the weight 1 part of $K_{0}(X)_{\mathbb{Q}}$. In particular, when $Y$ is a principal Cartier divisor, $[Y][Z]$ maps to zero in $C H_{|Z|}^{p+q}(X)_{\mathbb{Q}}$.

We shall denote by $R_{\text {fin }}^{p+q}(X)_{\mathbb{Q}}$ the space of $\mathbb{Q}$-cycles of the form $\sum_{i} q_{i} \operatorname{div}\left(f_{i}\right)$, where $q_{i} \in \mathbb{Q}$ and $f_{i} \in k\left(Y_{i}\right)^{*}$ is a nontrivial rational function on an integral subscheme $Y_{i}$ contained in some closed fiber of $\pi: X \rightarrow S$, of codimension $p+q-1$ in $X$. Observe that, for any cycle $R \in R_{\text {fin }}^{p+q}(X)_{\mathbb{Q}}$, the class of $(R, 0)$ in $\widehat{C H}^{p+q}(X)_{\mathbb{Q}}$ vanishes.

Now let $\alpha \in \widehat{C H}^{p}(X)$ and $\beta \in \widehat{C H}^{q}(X)$. By the moving lemma on $X_{K}$ we may represent $\alpha$ and $\beta$ by pairs $\left(Y, g_{Y}\right) \in \widehat{Z}^{p}(X)$ and $\left(Z, g_{Z}\right) \in \widehat{Z}^{q}(X)$ respectively, where $Y$ and $Z$ meet properly on $X_{K}$.

Let $Y . Z$ be any representative in $Z^{p+q}(|Y| \cap|Z|)_{\mathbb{Q}}$ of the product [Y]. [Z] in $C H_{|Y| \cap|Z|}^{p+q}(X)$. As $Y$ and $Z$ meet properly in $X_{K}$, it is a $\mathbb{Q}$-cycle well defined up to the addition of a $\mathbb{Q}$-cycle in $R_{\text {fin }}^{p+q}(X)_{\mathbb{Q}}$.

Let us write $g_{Y}=\eta+\partial u+\bar{\partial} v$, where $\eta$ is a Green form for $Y_{\mathbb{C}}$ in $\mathscr{D}\left(X_{\mathbb{R}}\right)$ of log type along $|Y|(\mathbb{C})$. We then define $\alpha \beta \in \widehat{C H}^{p+q}(X)_{\mathbb{Q}}$ to be the class of $\left(Y . Z, \eta * g_{Z}\right)$.

One has to show that this definition does not depend on choices. That it is so when $Y$ and $Z$ are fixed follows easily from the definition of $\widehat{C H}^{p+q}(X)$, from Proposition 1.3.1 and 1.3, Remark. To prove in general that it does not depend on the choices of representatives $\left(Y, g_{Y}\right)$ and $\left(Z, g_{Z}\right)$, we can use the commutativity of the product in the Chow groups with supports, the commutativity of the *-product, the "moving lemma for $K_{1}$-chains" ([G-S2], Lemma 4.2.6), and we are reduced to the following assertion (see [G-S2], top of page 144): let $W \subset X$ be an integral subscheme of codimension $p-1$ in $X, f \in k(W)^{*}$ a nonzero rational function on $W, Y=\operatorname{div}(f) \in Z^{p}(X)$ its divisor, and $g_{Y}=-\log |f|^{2}$ the canonical Green current for $Y$; assume that $Z \in Z^{q}(X)$ meets $Y$ properly on $X_{K}$, and let $g_{Z}$ be a Green current for $Z$; then the class of $\left(Y . Z, g_{Y} * g_{Z}\right)$ in $\widehat{C H}^{p+q}(X)_{\mathbb{Q}}$ vanishes.

To prove this, let us represent the element $[W][Z] \in C H_{|W| \cap|Z|}^{p+q-1}(X)_{\mathbb{Q}}$ by a $\mathbb{Q}$-cycle $\sum_{i \in I} n_{i} S_{i}+T$ on $|W| \cap|Z|$, where the $S_{i}$ 's are irreducible and meet 
$X_{K}$ while $T$ is supported on closed fibers. Since $Y$ and $Z$ meet properly on $X_{K}$, the function $f$ is regular at the generic point of $S_{i} ; i \in I$. By [G-S2] Lemma 4.2.5, there exist $u$ and $v$ in $\mathscr{D}(X(\mathbb{C}))$ such that

$$
\log |f|^{2} * g_{Z}=\log |f|^{2} \delta_{Z}=\sum_{i \in I} n_{i} \log \left|f_{\mid S_{i}}\right|^{2}+\partial u+\bar{\partial} v .
$$

Let us choose a rational function $\tilde{f}$ on $X$ which is regular at the generic point of $W$ and restricts to $f$ on $W$. Let $\widetilde{Y}=\operatorname{div}(\tilde{f})$. By the associativity of the intersection products for Chow groups with supports, we get the following equality in $C H_{|Y| \cap|Z|}^{p+q}(X)_{Q}$ :

$$
[Y][Z]=([\tilde{Y}][W])[Z]=[\tilde{Y}]([W][Z])=\sum_{i \in I} n_{i}[\tilde{Y}]\left[S_{i}\right]+[\tilde{Y}][T] .
$$

Since $\tilde{f}$ is regular at the generic point of $S_{i}$, we have

$$
[\widetilde{Y}]\left[S_{i}\right]=\left[\operatorname{div}\left(f_{\mid S_{i}}\right)\right] .
$$

On the other hand, since $\widetilde{Y}$ is principal and $T$ is supported by closed fibers, the image of $[\widetilde{Y}][T]$ in $C H_{|T|}^{p+q}(X)_{\mathbb{Q}}$ vanishes and any representative of $[\widetilde{Y}][T]$ in $Z^{p+q}(|Y| \cap|Z|)_{\mathbb{Q}}$ belongs to $R_{\text {fin }}^{p+q}(X)_{\mathbb{Q}}$. As $Y$ and $Z$ meet properly on $X_{K}$, (2.2.4) and (2.2.5) therefore imply

$$
Y . Z=\sum_{i \in I} n_{i} \operatorname{div}\left(f_{\mid S_{i}}\right) \quad \bmod R_{\mathrm{fin}}^{p+q}(X)_{\mathbb{Q}} .
$$

Finally, from (2.2.3), (2.2.6), we get the following equalities in $\widehat{C H}^{p+q}(X)_{\mathbb{Q}}$ :

$$
\begin{aligned}
& {\left[\left(Y . Z, g_{Y} * g_{Z}\right)\right]} \\
& \quad=\left[\left(\sum_{i \in I} n_{i} \operatorname{div} f_{\mid S_{i}},-\sum_{i \in I} n_{i} \log \left|f_{\mid S_{i}}\right|^{2}\right)\right]+[(0, \partial u+\bar{\partial} v)]=0 .
\end{aligned}
$$

2.3. The pairing $\widehat{C H}^{*}(X) \times Z_{*}(X) \rightarrow \widehat{C H}^{*}\left(\operatorname{Spec}\left(\mathscr{O}_{K}\right)\right)_{\mathbb{Q}}$.

2.3.1. Definition and basic properties. Let $X$ be a regular equidimensional projective arithmetic variety, $d$ its Krull dimension, $p$ and $q$ nonnegative integers, $Y \in Z_{p}(X)$, and $x \in \widehat{C H}^{q}(X)$. We shall define an element $(x \mid Y)$ in $\widehat{C H}_{p-q}(S)_{\mathbb{Q}}:=\widehat{C H}^{q-p+1}(S)_{\mathbb{Q}}$.

When $p$ is different from $q$ and $q+1$, then we let $(x \mid Y)=0$.

When $p=q$, choose a representative $(Z, g)$ of $x$ such that $Z$ meets $Y$ properly on $X_{K}$, i.e., $|Z|_{K} \cap|Y|_{K}$ is empty, and $g$ is smooth in some open neighborhood of $|Y|(\mathbb{C})$. The product $[Z]$. [Y] in $\mathrm{CH}_{|Z| \cap|Y|}^{d}(X)_{\mathbb{Q}}$ has a representative in $Z_{0}(|Z| \cap|Y|)_{\mathbb{Q}}$, supported on the closed fibers of $\pi: X \rightarrow S$, 
and the product of currents $g \delta_{Y}$ makes sense since their singular supports do not meet. Furthermore $g \delta_{Y}$ is closed for degree reasons. Therefore the pair $\left(Z . Y, g \delta_{Y}\right)$ defines a class in $\widehat{C H}^{d}(X)_{\mathbb{Q}}$ and we let

$$
(x \mid Y)=\pi_{*}\left[\left(Z . Y, g \delta_{Y}\right)\right]=\left[\left(\pi_{*}(Z . Y), \pi_{*}\left(g \delta_{Y}\right)\right)\right] \in \widehat{C H}^{1}(S)_{\mathbb{Q}} .
$$

When $p=q+1$ we let $z(Y) \in C H^{d-p}(X)$ be the class of $Y$ and $z(x) \in$ $C H^{q}(X)$ be as in 2.1.1. Then we define

$$
(x \mid Y)=\pi_{*}(z(x) z(Y)) \in C H^{0}(S)=\widehat{C H}^{0}(S)=\mathbb{Z} .
$$

In other words, $(x \mid Y)$ is the intersection number of the elements $z(x)_{K}$ and $\left[Y_{K}\right]$ in $C H^{*}\left(X_{K}\right)$, which have complementary dimensions.

Another definition of $(x \mid Y)$ is as follows. Let $g_{Y}$ be a Green current for $Y$ and $y \in \widehat{C H}^{d-p}(X)$ the class of $\left(Y, g_{Y}\right)$. Then, if $\omega(x) \in A^{q q}\left(X_{\mathbb{R}}\right)$ is the form attached to $X$ (see 2.1.1):

$$
(x \mid Y)=\pi_{*}\left(x y-a\left(\omega(x) g_{Y}\right)\right) \in \widehat{C H}^{*}(S)_{\mathbb{Q}} .
$$

Finally, we may give a third definition of $(x \mid Y)$. Assume that $Y$ is irreducible and let $\varphi: \widetilde{Y} \rightarrow Y$ be a projective birational morphism such that $\widetilde{Y}_{K}$ is smooth (which exists by resolution of singularities). Denote by $\tilde{\pi}: \widetilde{Y} \rightarrow S$ and $\psi: \tilde{Y} \rightarrow X$ the composite of $\varphi$ with $\pi$ and the inclusion $Y \hookrightarrow X$ respectively. Consider the cap-product $x \cap[\widetilde{Y}] \in \widehat{C H}_{p-q}(\widetilde{Y})_{\mathbb{Q}}$ of $x$ with the fundamental class of $\widetilde{Y}$ in $\widehat{C H}_{p}(\tilde{Y})=C H_{p}(\tilde{Y})$. Then

$$
(x \mid Y)=\tilde{\pi}_{*}(x \cap[\tilde{Y}])
$$

in $\widehat{C H}_{p-q}(S)_{\mathbb{Q}}=\widehat{C H}^{q-p+1}(S)_{\mathbb{Q}}($ compare $[\mathrm{Zh} 2])$.

Proposition 2.3.1. (i) Definitions (2.3.1), (2.3.2), (2.3.3), and (2.3.4) agree and do not depend on choices. They define a biadditive pairing

$$
\widehat{C H}^{q}(X) \otimes Z_{p}(X) \rightarrow \widehat{C H}^{q-p+1}(S)_{\mathbb{Q}}
$$

(ii) When $Y=\operatorname{div}(f)$, where $f$ is a nontrivial rational function on an integral subscheme contained in a closed fiber of $X$, then $(x \mid Y)=0$ for any $x \in \widehat{C H}^{*}(X)$.

(iii) For any $x \in \widehat{C H}^{*}(X)$, any $\eta \in \widehat{C H}^{*}(S)$, and any $Y \in Z_{*}(X)$, we have

$$
\left(x . \pi^{*}(\eta) \mid Y\right)=(x \mid Y) \eta \text { in } \widehat{C H}^{*}(S)_{\mathbb{Q}} .
$$

(iv) Let $f: X \rightarrow X^{\prime}$ be an $S$-morphism of regular projective arithmetic varieties, $x^{\prime} \in \widehat{C H}^{q}\left(X^{\prime}\right), Y \in Z_{p}(X)$, and $f_{*}(Y) \in Z_{p}\left(X^{\prime}\right)$ its direct image by $f$ ([Fu1] and [Fu2], 1.4 and Example 20.1.3). Then we have: 


$$
\left(f^{*}\left(x^{\prime}\right) \mid Y\right)=\left(x^{\prime} \mid f_{*}(Y)\right) \text { in } \widehat{C H}^{*}(S)_{\mathbb{Q}}
$$

(v) Let $f: X \rightarrow X^{\prime}$ be a flat $S$-morphism of regular projective arithmetic varieties, which has constant relative dimension $\delta$ and whose restriction to $X_{K}$ is smooth. Given $x \in \widehat{C H}^{q}(X), Y \in Z_{p}\left(X^{\prime}\right)$, and $f^{*}(Y) \in Z_{p+\delta}(X)$ its inverse image by $f([\mathrm{Fu} 1]$ and [Fu2], 1.7 and 20.1), we have:

$$
\left(x \mid f^{*}(Y)\right)=\left(f_{*}(x) \mid Y\right) \text { in } \widehat{C H}^{*}(S)_{\mathbb{Q}}
$$

(vi) Assume $Y \in Z_{p}(X)$ is irreducible. Let $\bar{L}$ be an hermitian line bundle on $X$ and $s$ any nonzero rational section of $L$ over $Y$. For any $x \in \widehat{C H}^{q}(X)$, we have:

$$
\left(x \widehat{c}_{1}(\bar{L}) \mid Y\right)=(x \mid \operatorname{div}(s))-\pi_{*} a\left(\omega(x) \log \|s\|^{2} \delta_{Y}\right) \text { in } \widehat{C H}^{*}(S)_{\mathbb{Q}} .
$$

Proof. To compare (2.3.1), (2.3.2), (2.3.3), and (2.3.4) we may assume that $Y$ is irreducible and that $p=q$ (when $p \notin\{q, q+1\}$, the comparison is trivial; when $p=q+1$, it easily follows from classical intersection theory for varieties over $K)$. By the projection formula for Chow groups with supports (cf. [G$\mathrm{S} 2]$, end of 4.4.3), $[Z][Y]$ coincides with $\varphi_{*}([Z] \cap[\tilde{Y}])$ in $C H_{|Y| \cap|Z|}^{d}(X)_{\mathbb{Q}}=$ $C H_{0}(|Y| \cap|Z|)_{\mathbb{Q}}$. Furthermore we may find a Green form of log type $\eta$ for $Z$ and some currents $u$ and $v$ which are smooth in a neighborhood of $Y(\mathbb{C})$ such that $g=\eta+\partial u+\bar{\partial} v$. It follows that

$$
g \delta_{Y}=\eta \delta_{Y}+\partial\left(u \delta_{Y}\right)+\bar{\partial}\left(v \delta_{Y}\right)=\psi_{*}\left[\varphi^{*}(\eta)\right]+\partial\left(u \delta_{Y}\right)+\bar{\partial}\left(v \delta_{Y}\right)
$$

Therefore the class of $\left([Z][Y], g \delta_{Y}\right)$ coincides with $\psi_{*}(x \cap[\tilde{Y}])$. Applying $\pi_{*}$ we conclude that (2.3.1) and (2.3.4) coincide. Furthermore $x y$ is by definition the class of $\left([Z][Y], \eta * g_{Y}\right)$ and

$$
\eta * g_{Y}=\eta \delta_{Y}+\omega(x) g_{Y}=g \delta_{Y}+\omega(x) g_{Y}-\partial\left(u \delta_{Y}\right)-\bar{\partial}\left(v \delta_{Y}\right),
$$

so that (2.3.3) and (2.3.1) are compatible. The rest of (i) is a consequence of these facts.

The statement (ii) is clear since $\delta_{Y}=0$ and the class of $Z . Y$ is zero in $C H_{|Z| \cap|Y|}^{p+q}(X)_{\mathbb{Q}}$, hence $Z . Y$ belongs to $R_{\text {fin }}^{p+q}(X)_{\mathbb{Q}}$.

Assertior (iii) follows from (2.3.3) together with the projection formula ([GS2], Theorem 4.4.3, 7)); indeed

$$
\begin{aligned}
\left(x \cdot \pi^{*} \eta \mid Y\right) & =\pi_{*}\left(x \cdot \pi^{*} \eta \cdot y-a\left(\omega\left(x \cdot \pi^{*} \eta\right) g_{Y}\right)\right) \\
& =\pi_{*}\left(x \cdot \pi^{*} \eta \cdot y-a\left(\omega(x) \cdot g_{Y}\right) \pi^{*} \eta\right) \quad \text { by }(2.1 .2) \\
& =\pi_{*}\left(x \cdot y-a\left(\omega(x) g_{Y}\right)\right) \cdot \pi^{*} \eta \quad \text { by the projection formula. }
\end{aligned}
$$


To prove (iv), we may again only consider the case $p=q$. Moreover, we may assume that $Y$ is irreducible. By the Moving Lemma for cycles in varieties over $K$, we may choose a representative $\left(Z^{\prime}, g^{\prime}\right)$ of $x^{\prime}$, where $Z^{\prime}$ is a cycle in $Z^{q}\left(X^{\prime}\right)$ such that $f^{-1}\left(\left|Z^{\prime}\right|\right)_{K}$ has codimension $q$ in $X_{K}$ and $\left|Z^{\prime}\right|$ does not meet $f(Y)$ in $X_{K}^{\prime}$. Moreover $g^{\prime}$ may be chosen to be a Green form of $\log$ type for $Z^{\prime}$. Let $Z$ be a representative in $Z^{q}\left(f^{-1}\left(\left|Z^{\prime}\right|\right)\right)$ of the cycle class $f^{*}\left(\left[Z^{\prime}\right]\right) \in C H_{f^{-1}\left(\left|Z^{\prime}\right|\right)}^{q}(X)$. Then $f^{*}\left(x^{\prime}\right)$ is the class of $\left(Z, f^{*}\left(g^{\prime}\right)\right)$ in $\widehat{C H}^{q}(X)$. As $|Z|_{K} \cap|Y|_{K}$ and $\left|Z^{\prime}\right|_{K} \cap|f(Y)|_{K}$ are empty, we can use definition (2.3.1) and we get

$$
\left(f^{*}\left(x^{\prime}\right) \mid Y\right)=\pi_{*}\left[\left(Z . Y, f^{*}\left(g^{\prime}\right) . \delta_{Y}\right)\right]
$$

and

$$
\left(x^{\prime} \mid f_{*}(Y)\right)=\pi_{*}^{\prime}\left[\left(Z^{\prime} \cdot f_{*}(Y), g^{\prime} \cdot \delta_{f_{*}(Y)}\right)\right],
$$

where $\pi^{\prime}$ is the structural morphism $X^{\prime} \rightarrow S$, and where $Z . Y$ and $Z^{\prime} . f_{*}(Y)$ denote representatives in $Z_{0}(|Z| \cap Y)$ and $Z_{0}\left(\left|Z^{\prime}\right| \cap f(Y)\right)$ of products in Chow groups with support. The projection formula for rational Chow groups with supports (see [G-S2], end of 4.4.3) shows that

$$
f_{*}(Z . Y)-Z^{\prime} \cdot f_{*}(Y) \in R_{\mathrm{fin}}^{d}(X)_{\mathbb{Q}}
$$

Moreover, as $g^{\prime}$ is smooth on a neighborhood of $Y(\mathbb{C})$, we have

$$
f_{*}\left(f^{*}\left(g^{\prime}\right) \cdot \delta_{Y}\right)=g^{\prime} \cdot f_{*}\left(\delta_{Y}\right)=g^{\prime} \cdot \delta_{f_{*}(Y)} .
$$

From this equality and from (2.3.9), it follows that

$$
f_{*}\left[\left(Z . Y, f^{*}\left(g^{\prime}\right) \cdot \delta_{Y}\right)\right]=\left[\left(Z^{\prime} \cdot f_{*}(Y), g^{\prime} \cdot \delta_{f_{*}(Y)}\right)\right] \text {. }
$$

This implies (2.3.7), since $\pi_{*}=\pi_{*}^{\prime} f_{*}$.

To prove (v) choose a Green current $g_{Y}$ for $Y$. Let $y$ be the class of $\left(Y, g_{Y}\right)$ and $\pi: X^{\prime} \rightarrow S$ the projection. Notice that $\omega\left(f_{*}(x)\right)=f_{*}(\omega(x))$ ([G-S2], 3.6). From (2.3.3) we get

$$
\left(f_{*}(x) \mid Y\right)=\pi_{*}\left[f_{*}(x) y-a\left(f_{*}(\omega(x)) g_{Y}\right)\right],
$$

and, by the projection formula

$$
\begin{aligned}
\left(f_{*}(x) \mid Y\right) & =\pi_{*}\left[f_{*}\left(x f^{*}(y)\right)-a f_{*}\left(\omega(x) f^{*}\left(g_{Y}\right)\right)\right] \\
& =\left(x \mid f^{*}(Y)\right)
\end{aligned}
$$

since $f^{*}(y)=\left(f^{*}(Y), f^{*}\left(g_{Y}\right)\right)$ by [G-S2], 3.6.1 and Theorem 4.4.3, 2).

To prove (vi) choose a rational section $\tilde{s}$ of $L$ over $X$ whose restriction to $Y$ coincides with $s$, let $g_{Y}$ be a Green current for $Y$, and $y$ be the class of $\left(Y, g_{Y}\right)$. From (2.3.3) we get

$$
\left(x \widehat{c}_{1}(\bar{L}) \mid Y\right)=\pi_{*}\left(\left(x \widehat{c}_{1}(\bar{L})\right) y-a\left(\omega\left(x \widehat{c}_{1}(\bar{L})\right) g_{Y}\right)\right) \text {. }
$$


By the associativity of the intersection product, we have

$$
\left(x \widehat{c}_{1}(\bar{L})\right) y=x\left(\widehat{c}_{1}(\bar{L}) y\right)=x\left[\left(\operatorname{div}(s),-\log \|\tilde{s}\|^{2} * g_{Y}\right)\right] \text {. }
$$

By definition, $-\log \|\tilde{s}\|^{2} * g_{Y}$ is equal to $-\log \|\tilde{s}\|^{2} \delta_{Y}+c_{1}(\bar{L}) g_{Y}$, and since $\omega\left(x \widehat{c}_{1}(\bar{L})\right)=\omega(x) c_{1}(\bar{L})$ we conclude from (2.3.3) that

$$
\left(x \widehat{c}_{1}(\bar{L}) \mid Y\right)=(x \mid \operatorname{div}(s))-\pi_{*} a\left(\omega(x) \log \|s\|^{2} \delta_{Y}\right) .
$$

Remarks. (i) If in definition (2.3.3) we use the pairing defined in [G-S2], 4.3.2, instead of the more general product of [G-S2], 4.2.3, we get an element $(x \mid Y)$ in $\widehat{C H}^{*}(S)$, i.e., we do not need to neglect torsion.

(ii) Suppose more generally that $X$ is a projective arithmetic variety which we allow to be singular away from the generic fiber $X_{K}$. Let $\bar{E}_{1}, \ldots, \bar{E}_{n}$ be hermitian vector bundles over $X$. For any sequence $q_{1}, \ldots, q_{n}$ of positive integers, and any cycle $X \in Z_{p}(X)$, we can define an element $\left(\widehat{c}_{q_{1}}\left(\bar{E}_{1}\right) \cdots \widehat{c}_{q_{n}}\left(\bar{E}_{n}\right) \mid Y\right)$ in $C H^{q-p+1}(S)_{\mathbb{Q}}$, where $q=q_{1}+\cdots+q_{n}$, by the following variant of (2.3.3): for any Green current $g_{Y}$ for $Y$, we let $y=$ $\left[\left(Y, g_{Y}\right)\right] \in \widehat{C H}_{p}(X)$ and

$$
\begin{aligned}
& \left(\widehat{c}_{q_{1}}\left(\bar{E}_{1}\right) \cdots \widehat{c}_{q_{n}}\left(\bar{E}_{n}\right) \mid Y\right) \\
& \quad=\pi_{*}\left(\widehat{c}_{q_{1}}\left(\bar{E}_{1}\right) \cap\left(\cdots \cap\left(\widehat{c}_{q_{n}}\left(\bar{E}_{n}\right) \cap y\right)\right)-a\left(c_{q_{1}}\left(\bar{E}_{1}\right) \cdots c_{q_{n}}\left(\bar{E}_{n}\right) g_{Y}\right)\right) .
\end{aligned}
$$

One easily checks that it does not depend on $g_{Y}$, and that when $p=q+1$ it coincides with the intersection number of $c_{q_{1}}\left(E_{1 K}\right) \cdots c_{q_{n}}\left(E_{n K}\right)$ and of $[Y]_{K}$ in $C H^{*}\left(X_{K}\right)$.

Since $X$ is quasi-projective over $S$, one may find vector bundles $F_{1}, \ldots, F_{n}$ over a projective arithmetic variety $X^{\prime}$ smooth over $S$, an $S$-morphism $f$ : $X \rightarrow X^{\prime}$, and isomorphisms $E_{i} \simeq f^{*} F_{i}$ (see [Fu1], §3-2). We can assume that $f$ is a closed imbedding by replacing $X^{\prime}$ by $X^{\prime} \times_{S} \mathbb{P}_{S}^{N}, f$ by $(f, i)$, where $i: X \hookrightarrow \mathbb{P}_{S}^{N}$ is an imbedding, and $F_{i}$ by $p r_{1}^{*} F_{i}$. Then the vector bundles $F_{i}$ may be endowed with hermitian metrics which make the isomorphisms $f^{*} F_{i} \simeq E_{i}$ isometric. By using variants of the arguments in the proof of Proposition 2.3.1 and in [G-S6], 2.3 and 2.4.2, one can prove the following formula:

$$
\left(\widehat{c}_{q_{1}}\left(\bar{E}_{1}\right) \cdots \widehat{c}_{q_{n}}\left(\bar{E}_{n}\right) \mid Y\right)=\left(\widehat{c}_{q_{1}}\left(\bar{F}_{1}\right) \cdots \widehat{c}_{q_{n}}\left(\bar{F}_{n}\right) \mid f_{*}(Y)\right) .
$$

This is easily seen to imply that assertions (ii), (iii), (iv), and (vi) of Proposition 2.3.1 still hold in this more general situation (more precisely, $X$ and $X^{\prime}$ are only supposed to be projective arithmetic varieties; $x$ is replaced by $\widehat{c}_{q_{1}}\left(\bar{E}_{1}\right) \cdots \widehat{c}_{q_{n}}\left(\bar{E}_{n}\right), \omega(x)$ by $c_{q_{1}}\left(\bar{E}_{1}\right) \cdots c_{q_{n}}\left(\bar{E}_{n}\right), x^{\prime}$ by $\widehat{c}_{q_{1}}\left({\overline{E^{\prime}}}_{1}\right) \cdots \widehat{c}_{q_{n}}\left(\bar{E}^{\prime}{ }_{n}\right)$, and $f^{*} x^{\prime}$ by $\widehat{c}_{q_{1}}\left(f^{*}{\overline{E^{\prime}}}_{1}\right) \cdots \widehat{c}_{q_{n}}\left(f^{*}{\overline{E^{\prime}}}_{n}\right)$, where $\bar{E}_{1}, \ldots, \bar{E}_{n}$ and ${\overline{E^{\prime}}}_{1}, \ldots,{\overline{E^{\prime}}}_{n}$ are hermitian vector bundles on $X$ and $X^{\prime}$ respectively). 
It is also possible to prove a formula analogous to (2.3.4). This allows one to make sense of $(x \mid Y) \in \widehat{C H}^{*}(S)_{\mathbb{Q}}$ when $x$ is a product of arithmetic Chern classes of hermitian vector bundles over an arbitrary arithmetic variety $X$, and when $Y$ is any cycle on $X$ such that $|Y|$ maps properly to $S$.

2.3.2. Arakelov varieties and arithmetic fundamental classes. Suppose now that $\bar{X}=(X, \mu)$ is an Arakelov variety, i.e., that $X$ is a projective arithmetic variety and that $\mu \in A^{1,1}\left(X_{\mathbb{R}}\right)$ is a Kähler form (cf. [G-S2], 5.1). Let $\mathscr{H}^{p p}\left(X_{\mathbb{R}}\right)$ be the space of forms in $A^{p p}\left(X_{\mathrm{R}}\right)$ which are harmonic with respect to $\mu$ and $H: \mathscr{D}^{p, p}\left(X_{\mathrm{R}}\right) \rightarrow \mathscr{H}^{p p}\left(X_{\mathrm{R}}\right)$ the harmonic projection. Given a cycle $Y \in Z^{p}(X)$ we may choose a Green current $g_{Y}$ such that $d d^{c} g_{Y}+\delta_{Y}=H\left(\delta_{Y}\right)$ and $H\left(g_{Y}\right)=0$. These two conditions determine $g_{Y}$ uniquely, up to the addition of a current of the form $\partial u+\bar{\partial} v$ ([G-S2], 5.1) and any such $g_{Y}$ will be called a $\mu$-normalized Green current for $Y$. We let

$$
[Y]_{\mu}=\left(Y, g_{Y}\right) \in \widehat{C H}^{d-p}(X)
$$

(see also $[\mathrm{Fa} 2]$, where $[Y]_{\mu}$ is denoted $\widehat{Y}$, and [B2], 2.6). The class $[Y]_{\mu}$ will be called the arithmetic fundamental class of the cycle $Y$ on the Arakelov variety $(X, \mu)$.

Notice that the normalization conditions on $g_{Y}$ may be written as:

$$
d d^{c} g_{Y}+\delta_{Y} \in \mathscr{H}^{p, p}\left(X_{\mathbb{R}}\right)
$$

and

$$
\int_{X(\mathbb{C})} \omega \cdot g_{Y}=0
$$

for any $\omega \in \mathscr{H}^{d-p, d-p}\left(X_{\mathbb{R}}\right)$. Therefore $g_{Y}$ and $[Y]_{\mu}$ depend only on the space of harmonic forms associated to the Kähler structure defined by $\mu$. In particular they are unchanged if $\mu$ is replaced by $\lambda \mu, \lambda \in \mathbb{R}_{+}^{*}$. Furthermore $[Y]_{\mu}=0$ if $Y$ is the divisor of a rational function on a subvariety contained in a closed fiber of $X$ over $S$. Finally, from definition (2.3.3) we get:

Lemma 2.3.2. For any $x \in \widehat{C H}^{q}(X)$ such that $\omega(x)$ is harmonic with respect to $\mu$,

$$
(x \mid Y)=\pi_{*}\left(x \cdot[Y]_{\mu}\right) \text { in } \widehat{C H}^{q-p+1}(S)_{\mathbb{Q}}
$$

2.3.3. External products. Let $X_{1}$ and $X_{2}$ be two arithmetic varieties which are smooth over $S$, and $X=X_{1} \times_{S} X_{2}$ their product. Given $Y_{1} \in Z_{p_{1}}(X)$ and $Y_{2} \in Z_{p_{2}}(X)$, their external product $Y=Y_{1} \times Y_{2} \in Z_{p_{1}+p_{2}-1}(X)$ may be defined as in [Fu2], 20.2. Namely, $Y_{1} \times Y_{2}$ is zero when both cycles are supported in a closed fiber. If $Y_{1}$ say is integral and flat over $S$, then the morphism $\left|Y_{1}\right| \times_{S} X_{2} \rightarrow X_{2}$ is flat, and $Y_{1} \times Y_{2}$ is obtained as the direct image by the inclusion $\left|Y_{1}\right| \times_{S} X_{2} \rightarrow X_{1} \times_{S} X_{2}$ of the pull-back of $Y_{2} \in Z_{p_{2}}\left(X_{2}\right)$ by this flat morphism. 
Let $f_{1}: X \rightarrow X_{1}$ and $f_{2}: X \rightarrow X_{2}$ be the two projections. When $x_{1} \in$ $\widehat{C H}^{q_{1}}\left(X_{1}\right)$ and $x_{2} \in \widehat{C H}^{q_{2}}\left(X_{2}\right)$ we let

$$
x_{1} \times x_{2}=f_{1}^{*}\left(x_{1}\right) f_{2}^{*}\left(x_{2}\right) \in \widehat{C H}^{q_{1}+q_{2}}(X) .
$$

Proposition 2.3.3. Under the above hypotheses

$$
\left(x_{1} \times x_{2} \mid Y_{1} \times Y_{2}\right)=\left(x_{1} \mid Y_{1}\right)\left(x_{2} \mid Y_{2}\right) \text { in } \widehat{C H}^{*}(S) \text {. }
$$

Proof. Since $X_{1}$ and $X_{2}$ are smooth over $S$, we need not tensor $\widehat{C H}^{*}(S)$ by $\mathbb{Q}\left(\right.$ see 2.1.1). Let $\pi_{1}: X_{1} \rightarrow S, \pi_{2}: X_{2} \rightarrow S, \pi: X \rightarrow S$ be the obvious projections. Both sides of (2.3.12) vanish unless $\left(p_{1}, p_{2}\right)$ is equal to $\left(q_{1}+1, q_{2}+1\right),\left(q_{1}+1, q_{2}\right)$, or $\left(q_{1}, q_{2}+1\right)$.

In the first case we have

$$
\left(x_{1} \times x_{2} \mid Y_{1} \times Y_{2}\right)=\pi_{*}\left(z\left(x_{1} \times x_{2}\right) z\left(Y_{1} \times Y_{2}\right)\right) \text {. }
$$

Notice that

$$
z\left(x_{1} \times x_{2}\right)=f_{1}^{*}\left(z\left(x_{1}\right)\right) f_{2}^{*}\left(z\left(x_{2}\right)\right)
$$

and

$$
z\left(Y_{1} \times Y_{2}\right)=f_{1}^{*}\left(z\left(Y_{1}\right)\right) f_{2}^{*}\left(z\left(Y_{2}\right)\right) .
$$

From the projection formula it follows that

$$
\left(x_{1} \times x_{2} \mid Y_{1} \times Y_{2}\right)=\pi_{1 *}\left(z\left(x_{1}\right) z\left(Y_{1}\right)\right) \pi_{2 *}\left(z\left(x_{2}\right) z\left(Y_{2}\right)\right)=\left(x_{1} \mid Y_{1}\right)\left(x_{2} \mid Y_{2}\right) \text {. }
$$

Assume now that $\left(p_{1}, p_{2}\right)=\left(q_{1}+1, q_{2}\right)$ (the case $\left(p_{1}, p_{2}\right)=\left(q_{1}, q_{2}+1\right)$ follows by symmetry). Let

$$
n=\left(x_{1} \mid Y_{1}\right)=\pi_{1 *}\left(z\left(x_{1}\right) z\left(Y_{1}\right)\right) \in \widehat{C H}^{0}(S)=\mathbb{Z}
$$

Choose a representative $\left(Z_{1}, g_{1}\right)$ for $x_{1}$ such that $Z_{1}$ meets $Y_{1}$ properly on $X_{1 K}$ and $g_{1}=\left[\eta_{1}\right]$, where $\eta_{1}$ is a Green form of log type for $Y_{1}$. Choose similarly a representative $\left(Z_{2}, g_{2}\right)$ for $x_{2}$. The external product $x_{1} \times x_{2}$ is then represented by $\left(Z_{1} \times Z_{2}, g_{1} \wedge \delta_{Z_{2}}+\omega_{1} \wedge g_{2}\right)$, where $\omega_{1}=d d^{c} g_{1}+\delta_{Z_{1}}$ and $\wedge$ is the external product of currents.

Since, by hypothesis, $Z_{2}(\mathbb{C})$ and $Y_{2}(\mathbb{C})$ do not meet, the current $g_{1} \wedge \delta_{Z_{2}}$ vanishes in a neighborhood of $\left|Y_{1} \times Y_{2}\right|(\mathbb{C})$. Therefore $g=g_{1} \wedge \delta_{Z_{2}}+\omega_{1} \wedge g_{2}$ is a Green current for $Z_{1} \times Z_{2}$ which is smooth on $\left|Y_{1} \times Y_{2}\right|(\mathbb{C})$. By (2.3.1) we know that $\left(x_{1} \times x_{2} \mid Y_{1} \times Y_{2}\right)$ is the class of $\left(\pi_{*}\left(\left(Z_{1} \times Z_{2}\right) \cdot\left(Y_{1} \times Y_{2}\right)\right), \pi_{*}\left(g \delta_{Y_{1} \times Y_{2}}\right)\right)$. From the projection formula for Chow groups with supports we get the following equality in $Z_{0}(S)$ :

$$
\pi_{*}\left(\left(Z_{1} \times Z_{2}\right)\left(Y_{1} \times Y_{2}\right)\right)=\pi_{1 *}\left(Z_{1} . Y_{1}\right) \pi_{2 *}\left(Z_{2} . Y_{2}\right)=n \pi_{2 *}\left(\left[Z_{2}\right]\left[Y_{2}\right]\right) .
$$

On the other hand we have

$$
\pi_{*}\left(g \delta_{Y_{1} \times Y_{2}}\right)=\pi_{*}\left(\left(\omega_{1} \wedge g_{2}\right) \delta_{Y_{1} \times Y_{2}}\right)=n \pi_{2 *}\left(g_{2} \delta_{Y_{2}}\right)
$$


because $n=\pi_{1 *}\left(\omega_{1} \delta_{Y_{1}}\right)$, since the cohomology class of $\omega_{1}$ (resp. $\left.\delta_{Y_{1}}\right)$ is the fundamental class of $Z_{1}$ (resp. $Y_{1}$ ). From this we conclude that

$$
\left(x_{1} \times x_{2} \mid Y_{1} \times Y_{2}\right)=n\left(x_{2} \mid Y_{2}\right)=\left(x_{1} \mid Y_{1}\right)\left(x_{2} \mid Y_{2}\right)
$$

Remark. Let $X_{1}, X_{2}, X_{1}^{\prime}$, and $X_{2}^{\prime}$ be arithmetic varieties which are smooth over $S$, let $f_{1}: X_{1} \rightarrow X_{1}^{\prime}, f_{2}: X_{2} \rightarrow X_{2}^{\prime}$ be $S$-morphisms, and let $f_{1} \times$ $f_{2}: X_{1} \times{ }_{S} X_{2} \rightarrow X_{1}^{\prime} \times_{S} X_{2}^{\prime}$ be their product. Then for for any $\left(x_{1}, x_{2}\right)$ in $\widehat{C H}^{*}\left(X_{1}^{\prime}\right) \times \widehat{C H}^{*}\left(X_{2}^{\prime}\right)$ the identity

$$
\left(f_{1} \times f_{2}\right)^{*}\left(x_{1} \times x_{2}\right)=f_{1}^{*}\left(x_{1}\right) \times f_{2}^{*}\left(x_{2}\right)
$$

holds in $\widehat{C H}^{*}\left(X_{1} \times{ }_{S} X_{2}\right)$. If moreover $f_{1}$ and $f_{2}$ are smooth over the generic fibers and proper, for any $\left(x_{1}, x_{2}\right)$ in $\widehat{C H}^{*}\left(X_{1}\right) \times \widehat{C H}^{*}\left(X_{2}\right)$, the identity

$$
\left(f_{1} \times f_{2}\right)_{*}\left(x_{1} \times x_{2}\right)=f_{1 *}\left(x_{1}\right) \times f_{2 *}\left(x_{2}\right)
$$

holds in $\widehat{C H}^{*}\left(X_{1}^{\prime} \times_{S} X_{2}^{\prime}\right)$. This follows from the functoriality of the pull-back and from the projection formula.

2.3.4. Degrees. Let $X$ be a regular projective arithmetic variety, $Y \in Z_{p}(X)$, and $x \in \widehat{C H}^{q}(X)$. To the element $(x \mid Y)$ in $\widehat{C H}^{*}(S)_{\mathbb{Q}}$ we can attach both an integer and a real number. Namely, with the notations of 2.1.3, we define

and

$$
\operatorname{deg}_{K}(x \mid Y)=\operatorname{deg}_{K}((x \mid Y)) \in \mathbb{Z}
$$

$$
\widehat{\operatorname{deg}}(x \mid Y)=\widehat{\operatorname{deg}}((x \mid Y)) \in \mathbb{R} .
$$

Let $z(x)_{K} \in C H^{q}\left(X_{K}\right)$ and $z(Y)_{K} \in C H^{d-p}\left(X_{K}\right)$ be the images of $x$ and $Y$ in the algebraic Chow groups of $X_{K}$. Then

$$
\begin{aligned}
\operatorname{deg}_{K}(x \mid Y) & =\pi_{*}(z(x) \cdot[Y]) \\
& =\pi_{K *}\left(z(x)_{K} \cdot\left[Y_{K}\right]\right) \in C H^{0}(\operatorname{Spec}(K))=\mathbb{Z}=\widehat{C H}^{0}(S)
\end{aligned}
$$

is the usual intersection number of the cycle classes on $X_{K}$ attached to $x$ and $Y$. If $x$ is a class in $C H^{*}(X)$ or in $C H^{*}\left(X_{K}\right)$, we will still use the notation $\operatorname{deg}_{K}(x \mid Y)$ for this intersection number.

We can give "explicit" formulae for $\widehat{\operatorname{deg}}(x \mid Y)$ as follows. Let $(Z, g)$ be a representative of $x$ in $\widehat{Z}^{q}(X)$ such that $|Z|_{K} \cap|Y|_{K}=\varnothing$ and $g$ is smooth near $|Y|(\mathbb{C})$. If $\sum_{\alpha} m_{\alpha} W_{\alpha}$ is a cycle (with rational coefficients) supported in $|Z| \cap|Y|$ representing $[Z][Y]$, and if $k\left(W_{\alpha}\right)$ is the residue field of the closed point $W_{\alpha}$, we have from formulae (2.1.8) and (2.3.1):

$$
\widehat{\operatorname{deg}}(x \mid Y)=\sum_{\alpha} m_{\alpha} \log \left(\# k\left(W_{\alpha}\right)\right)+\frac{1}{2} \int_{X(\mathbb{C})} g \delta_{Y} .
$$


In particular $\widehat{\operatorname{deg}}(x \mid Y)$ depends only on $X$ as an arithmetic variety over $\operatorname{Spec}(\mathbb{Z})$, and not on the map $\pi: X \rightarrow S$.

Assume moreover that $Y$ and $Z$ are irreducible (by bilinearity of $(x \mid Y)$, one easily reduces to this case). As $Z$ and $Y$ do not meet on $X_{K}$, the coherent sheaves $\operatorname{Tor}_{i}^{\mathscr{O}_{X}}\left(\mathscr{O}_{Z}, \mathscr{O}_{Y}\right)$ are supported by closed fibers of $X$, hence have finite cohomology groups. It follows from [G-S2], 4.3.8 iv)-v), (2.1.8), and (2.3.1) that

$$
\widehat{\operatorname{deg}}(x \mid Y)=\sum_{i, j \geq 0}(-1)^{i+j} \log \# H^{i}\left(X, \operatorname{Tor}_{j}^{\mathscr{O}_{X}}\left(\mathscr{O}_{Z}, \mathscr{O}_{Y}\right)\right)+\frac{1}{2} \int_{X(\mathbb{C})} g \delta_{Y} .
$$

Properties of the pairing $(x \mid Y)$ imply properties of $\operatorname{deg}_{K}(x \mid Y)$ and $\widehat{\operatorname{deg}}(x \mid Y)$. For instance Proposition 2.3.3, (2.1.9), and (2.1.10) give

$$
\operatorname{deg}_{K}\left(x_{1} \times x_{2} \mid Y_{1} \times Y_{2}\right)=\operatorname{deg}_{K}\left(x_{1} \mid Y_{1}\right) \operatorname{deg}_{K}\left(x_{2} \mid Y_{2}\right)
$$

and

$$
\begin{aligned}
\widehat{\operatorname{deg}}\left(x_{1} \times x_{2} \mid Y_{1} \times Y_{2}\right)= & \widehat{\operatorname{deg}}\left(x_{1} \mid Y_{1}\right) \operatorname{deg}_{K}\left(x_{2} \mid Y_{2}\right) \\
& +\operatorname{deg}_{K}\left(x_{1} \mid Y_{1}\right) \widehat{\operatorname{deg}}\left(x_{2} \mid Y_{2}\right),
\end{aligned}
$$

and Proposition 2.3.1, (iii) implies, for any $x \in \widehat{C H}^{p-1}(X), \eta \in \widehat{C H}^{1}(S)$, and $Z \in Z_{p}(X)$,

$$
\widehat{\operatorname{deg}}\left(x \cdot \pi^{*}(\eta) \mid Z\right)=\operatorname{deg}_{K}(x \mid Z) \cdot \widehat{\operatorname{deg}}(\eta)
$$

\section{THE HEIGHT OF CYCLES DEFINED BY AN HERMITIAN LINE BUNDLE}

\subsection{Definition and examples.}

3.1.1. The height $h_{\bar{L}}$. Let $X$ be a regular projective arithmetic variety and $\bar{L}=(L, h)$ an hermitian line bundle on $X$. For any cycle $Z \in Z_{p}(X)$ we define the height of $Z$ with respect to $\bar{L}$ to be the real number

$$
h_{\bar{L}}(Z)=\widehat{\operatorname{deg}}\left(\widehat{c}_{1}(\bar{L})^{p} \mid Z\right) \in \mathbb{R}
$$

where $\widehat{c}_{1}(\bar{L}) \in \widehat{C H}^{1}(X)$ is the first Chern class of $\bar{L}(1.2 .2)$ and $\widehat{\mathrm{deg}}$ is defined as in 2.3.4 (this extends the definition in [B2], $\S \S 2.4-2.5$ ). This is the arithmetic counterpart of the degree

$$
\begin{aligned}
\operatorname{deg}_{L_{K}}(Z) & =\pi_{K *}\left(c_{1}\left(L_{K}\right)^{p-1}\left[Z_{K}\right]\right)=\operatorname{deg}_{K}\left(\widehat{c}_{1}(\bar{L})^{p-1} \mid Z\right) \\
& \in C H^{0}(\operatorname{Spec}(K)) \simeq \mathbb{Z},
\end{aligned}
$$

where $\pi_{K}$ is the structural morphism $X_{K} \rightarrow \operatorname{Spec}(K), c_{1}\left(L_{K}\right) \in C H^{1}\left(X_{K}\right)$ is the first Chern class of the restriction of $L$ to $X_{K},\left[Z_{K}\right] \in C H_{p-1}\left(X_{K}\right)$ is the class of the restriction of $Z$ to $X_{K}$, and $\operatorname{deg}_{K}$ is defined as in 2.3.4. 
Notice that these definitions extend to the case where $X$ is an arbitrary arithmetic variety and $|Z|$ is proper over $S$ (use 2.3.1, Remark (ii); see also [Zh2] and infra 3.2.1, Remark).

Finally, when $Z$ is a cycle of dimension $q$ on $X_{K}$ we will denote by $h_{\bar{L}}(Z)$ and $\operatorname{deg}_{L_{K}}(Z)$ the height and the degree of its Zariski closure $\bar{Z}$ in $X$; and if $M_{K}$ is a line bundle over $X_{K}$, we will denote by $\operatorname{deg}_{M_{K}} Z$ the intersection number of $c_{1}\left(M_{K}\right)^{q}$ and [Z] in $C H^{*}\left(X_{K}\right)$.

\subsubsection{Examples.}

3.1.2.1. When $X=S$ we have $h_{\bar{L}}(S)=\widehat{\operatorname{deg}}(\bar{L})$ (see 2.1.3). If $X$ is any projective arithmetic variety and $P \in X(K)$ is a rational point on $X$, and if $\varepsilon_{P}: S \rightarrow X$ denotes the section of $\pi$ attached to $P$, we have by (2.1.12) and (2.3.6):

$$
h_{\bar{L}}(P)=\widehat{\operatorname{deg}}\left(\widehat{c}_{1}(\bar{L}) \mid \varepsilon_{P}(S)\right)=\widehat{\operatorname{deg}} \varepsilon_{P}^{*}(\bar{L}) .
$$

3.1.2.2. Consider a closed point of $S$, i.e., a nonzero prime ideal $\wp$ in $\mathscr{O}_{K}$. Let $\mathbb{F}_{\wp}=\mathscr{O}_{K} / \wp$ be its residue field and let $N_{\wp}=\# \mathbb{F}_{\wp}$ be its norm. Let $Z \in$ $Z_{p}(X)$ be a cycle on $X$ the support of which is contained in the closed subset $X_{\mathrm{F}_{p}}=\pi^{-1}(\{\wp\}) \subset X$. Let $[Z]_{\mathbb{F}_{p}} \in C H_{p}\left(X_{\mathbb{F}_{p}}\right)$ be the class of $Z$, and $\pi_{\mathbb{F}_{p}}$ : $X_{\mathbb{F}_{p}} \rightarrow \operatorname{Spec}\left(\mathbb{F}_{\wp}\right)$ the projection. Then

$$
h_{\bar{L}}(Z)=\pi_{\mathbb{F}_{p^{*}}}\left(c_{1}\left(L_{\mathbb{F}_{\mathfrak{p}}}\right)^{p} \cap[Z]_{\mathbb{F}_{p}}\right) \log N \wp \in \mathbb{R},
$$

where $\pi_{\mathbb{F}_{p^{*}}}\left(c_{1}\left(L_{\mathbb{F}_{\mathfrak{p}}}\right)^{p} \cap[Z]_{\mathbb{F}_{\mathfrak{p}}}\right)\left(\in C H_{0}\left(\operatorname{Spec}\left(\mathbb{F}_{\wp}\right)\right)=\mathbb{Z}\right)$ is the usual degree of $Z$ with respect to $L_{\mathbb{F}_{p}}$ on $X_{\mathbb{F}_{p}}$. To check this, notice that the composite map

$$
C H_{0}\left(\operatorname{Spec}\left(\mathbb{F}_{\wp}\right)\right) \rightarrow \widehat{C H}_{0}(S) \stackrel{\widehat{\operatorname{deg}}}{\longrightarrow} \mathbb{R}
$$

is just multiplication by $\log N_{\wp}$.

In particular, when $Z=\sum_{i} n_{i} P_{i} \in Z_{0}(X)$, where $n_{i} \in \mathbb{Z}$ and $P_{i}$ are closed points of $X$, the residue fields $k\left(P_{i}\right)$ are finite and

$$
h_{\bar{L}}(Z)=\sum_{i} n_{i} \log \left(\# k\left(P_{i}\right)\right) .
$$

In fact $h_{\bar{L}}(Z)$ equals by definition $\widehat{\operatorname{deg}}(1 \mid Z)$, and (3.1.4) follows immediately from (2.3.16).

3.1.2.3. Assume $\bar{E}$ is an hermitian vector bundle on $S$. Let

$$
\mathbb{P}(E)=\operatorname{Proj}\left(\operatorname{Sym}\left(E^{\vee}\right)\right)
$$

be the projective space of $E$ and $\mathscr{O}(1)$ the canonical quotient line bundle on $\mathbb{P}(E)$. We endow $\mathscr{O}(1)$ with the quotient metric, which is such that the projection $\pi^{*} E_{\mathbb{C}}^{\vee} \rightarrow \mathscr{O}(1)_{\mathbb{C}}$ induces an isometry between the orthogonal complement 
to its kernel and $\mathscr{O}(1)_{\mathbb{C}}$. For any cycle $Z \in Z_{p}(\mathbb{P}(E))$ we may then consider the height

$$
h_{F}(Z)=h_{\overline{\mathscr{O}(1)}}(Z) \text {. }
$$

When $\bar{E}=\overline{\mathscr{O}}_{S}^{N+1}$ is the trivial hermitian vector bundle of rank $N+1$ on $S$, this height coincides with the height introduced by Faltings in [Fa2], as follows from (2.3.11) with $\mu=c_{1}(\overline{\mathscr{O}(1)})$.

In particular, when $Z$ is the cycle attached as above to a rational point $P \in$ $\mathbb{P}^{N}(K)$, one recovers the classical height of $P$ ([Si], Zweiter Teil, $\S 2$; [No]; [We2] pp. 425-426; these authors use the $\ell^{1}$ or the $\ell^{\infty}$-norm instead of the $\ell^{2}$-norm on $\left.\mathbb{C}^{N+1}\right)$. Namely, if $\left(x_{0}, \ldots, x_{N}\right) \in K^{N+1}$ are homogeneous coordinates of $P$, we get

$$
h_{F}(P)=\sum_{\wp} \log \left(\max _{0 \leq i \leq N}\left|x_{i}\right|_{\wp}\right)+\sum_{\sigma} \log \sqrt{\sum_{0 \leq i \leq N}\left|\sigma\left(x_{i}\right)\right|^{2}},
$$

where $\wp$ runs over all nonzero prime ideals of $\mathscr{O}_{K},|\alpha|_{\wp}=\left(N_{\wp}\right)^{-v_{\wp}(\alpha)}$, where $v_{\wp}$ is the $\wp$-adic valuation, and $\sigma$ runs over all imbeddings of $K$ in $\mathbb{C}$.

Let $F$ be a subbundle ${ }^{3}$ of $E$, endowed with the induced hermitian metric. It follows from (3.2.1) below that, for any cycle $Z$ on $\mathbb{P}(F)$, the Faltings heights of $Z$ considered as a cycle in $\mathbb{P}(F)$ and as a cycle in $\mathbb{P}(E)$ coincide.

3.1.3. Multiheights. More generally, a finite sequence $\overline{\mathbf{L}}=\left(\bar{L}_{1}, \ldots, \bar{L}_{k}\right)$ of hermitian line bundles on a projective arithmetic variety $X$ allows one to define multiheights of the cycles on $X$. Namely, for any $p \in \mathbb{N}$ and any $I=\left(i_{1}, \ldots, i_{k}\right) \in \mathbb{N}^{k}$ such that $|I|:=i_{1}+\cdots+i_{k}=p$, we define the $I$-th multiheight of $Z \in Z_{p}(X)$ with respect to $\overline{\mathbf{L}}$ to be the real number

$$
h_{\overline{\mathbf{L}}}^{I}(Z)=\widehat{\operatorname{deg}}\left(\widehat{c}_{1}\left(\bar{L}_{1}\right)^{i_{1}} \cdots \widehat{c}_{1}\left(\bar{L}_{k}\right)^{i_{k}} \mid Z\right) .
$$

We can also define, for any $Z \in Z_{p+1}(X)$, the $I$-th multidegree

$$
\operatorname{deg}_{\mathbf{L}_{K}}^{I}(Z)=\operatorname{deg}_{K}\left(c_{1}\left(L_{1}\right)^{i_{1}} \cdots c_{1}\left(L_{k}\right)^{i_{k}} \mid Z\right) .
$$

The various properties of heights which are proved in the next sections have generalizations involving multiheights and multidegrees, which we leave to the reader (see also $[\mathrm{Gu}]$ ).

3.1.4. Change of ground ring. Let $K^{\prime}$ be a number field containing $K$, and $S^{\prime}=\operatorname{Spec}\left(\mathscr{O}_{K^{\prime}}\right)$. For any projective arithmetic variety $X$ over $S$ and any hermitian line bundle $\bar{L}$ on $X$, using the base change $S^{\prime} \rightarrow S$ we get an arithmetic variety $X^{\prime}=X \times_{S} S^{\prime}$ over $S^{\prime}$, and, if $f: X^{\prime} \rightarrow X$ is the projection

\footnotetext{
${ }^{3}$ I.e., $F$ is a subsheaf of $E$ such that $E / F$ is locally free; any such $F$ is determined by the $K$-vector space $F_{K} \subset E_{K}$.
} 
onto the first factor, we may consider the hermitian line bundle $\overline{L^{\prime}}:=f^{*} \bar{L}$ over $X^{\prime}$.

From formula (2.3.6) and 2.3.1, Remark (ii), it follows that for any $Z^{\prime} \in$ $Z_{*}\left(X^{\prime}\right)$ :

$$
h_{\overline{L^{\prime}}}\left(Z^{\prime}\right)=h_{\bar{L}}\left(f_{*}\left(Z^{\prime}\right)\right)
$$

This reduces the computation of heights of cycles on $X^{\prime}$ to the computation of heights of cycles on $X$. As $f$ is a finite and flat morphism of degree $\left[K^{\prime}: K\right]$, for any $Z \in Z_{*}(X)$, the pull-back $f^{*}(Z) \in Z_{*}\left(X^{\prime}\right)$ is well defined and the following identity holds in $Z_{*}(X)$ :

$$
f_{*} f^{*}(Z)=\left[K^{\prime}: K\right] Z \text {. }
$$

Therefore, we get from (3.1.7):

$$
h_{\overline{L^{\prime}}}\left(f^{*}(Z)\right)=\left[K^{\prime}: K\right] h_{\bar{L}}(Z) .
$$

Identities (3.1.7) and (3.1.8) still hold when $Z$ and $Z^{\prime}$ are cycles on $X_{K}$ and $X_{K^{\prime}}^{\prime}\left(\simeq X_{K} \otimes_{K} K^{\prime}\right)$. This allows one to define a normalized height $h_{\bar{L} \text {, norm }}$ on $Z_{*}\left(X_{\bar{Q}}\right)$ in the following way: for any cycle $Z \in Z_{*}\left(X_{\overline{\mathbb{Q}}}\right)$, there exists a number field $K^{\prime}$ such that $K \subset K^{\prime} \subset \overline{\mathbb{Q}}$ and such that $Z$ is defined over $K^{\prime}$, i.e., $Z$ is deduced through the scalar extension $K^{\prime} \hookrightarrow \overline{\mathbb{Q}}$ from a cycle $Z^{\prime} \in Z_{*}\left(X_{K^{\prime}}\right)$. Then we let

$$
h_{\bar{L}, \text { norm }}(Z)=\frac{1}{\left[K^{\prime}: \mathbb{Q}\right]} h_{\bar{L}}\left(f_{*}\left(\overline{Z^{\prime}}\right)\right) \text {. }
$$

By (3.1.7), this is also equal to $\left[K^{\prime}: \mathbb{Q}\right]^{-1} h_{\overline{L^{\prime}}}\left(\overline{Z^{\prime}}\right)$, and it easily follows from (3.1.8) that this number does not depend on the choice of $K^{\prime}$.

Observe finally that $\operatorname{deg}_{L_{K^{\prime}}^{\prime}}$ and $\operatorname{deg}_{L_{K}}$ satisfy the following compatibility formulae:

$$
\operatorname{deg}_{L_{K}} f_{*}\left(Z^{\prime}\right)=\left[K^{\prime}: K\right] \operatorname{deg}_{L_{K^{\prime}}}\left(Z^{\prime}\right)
$$

and

$$
\operatorname{deg}_{L_{K^{\prime}}^{\prime}} f^{*}(Z)=\operatorname{deg}_{L_{K}}(Z)
$$

3.2. Properties of $h_{\bar{L}}$. This section is devoted to the proof of various properties of heights of cycles which are classical in the case of cycles defined by rational points ([No]; [We2]; [Sz], 3.1).

3.2.1. Basic identities. Proposition 2.3.1 implies the following properties of the height of cycles:

Proposition 3.2.1. Let $\bar{L}$ be a hermitian line bundle over a regular projective arithmetic variety $X$.

(i) The height $h_{\bar{L}}(Z)$ is additive in $Z$. For any integer $n \in \mathbb{Z}$ and $Z \in$ $Z_{p}(X)$, one has 


$$
\operatorname{deg}_{L_{K}^{n}}(Z)=n^{p-1} \operatorname{deg}_{L_{K}}(Z) \text { and } h_{\bar{L}^{n}}(Z)=n^{p} h_{\bar{L}}(Z) \text {, }
$$

where $\bar{L}^{n}$ is the $n$-th tensor power of $\bar{L}$.

(ii) When $Z$ is the divisor of a rational function on an integral variety contained in a closed fiber of $X, h_{\bar{L}}(Z)=0$.

(iii) For any morphism $f: X \rightarrow X^{\prime}$ of regular arithmetic projective varieties and any cycle $Z$ on $X$,

$$
h_{f^{*}(\bar{L})}(Z)=h_{\bar{L}}\left(f_{*}(Z)\right)
$$

(iv) Assume that $Z \in Z_{p}(X)$ is irreducible, and let $s$ be a nonzero rational section of a power $L^{n}$ of $L$ over $Z, n \in \mathbb{Z}$, and \|\| the norm on $L^{n}$ deduced from $h$ by tensor power. Then

$$
h_{\bar{L}}(\operatorname{div}(s))=n h_{\bar{L}}(Z)+\int_{X(\mathbb{C})} \log \|s\| c_{1}(\bar{L})^{p-1} \delta_{Z}
$$

Remark. By appealing to 2.3.1, Remark (ii), we may extend Proposition 3.2.1 to the case where $X$ is a not necessarily regular arithmetic projective variety. This can also be done as follows, without any explicit reference to the operational Chern classes (2.1.7).

Let $\bar{L}$ be an hermitian line bundle over a projective arithmetic variety $X$. There exists an hermitian line bundle $\overline{L^{\prime}}$ over an arithmetic variety $X^{\prime}$ smooth over $S$ and an $S$-morphism $f: X \rightarrow X^{\prime}$, which is a closed imbedding such that $\bar{L} \simeq f^{*} \overline{L^{\prime}}$ (cf. 2.3.1, Remark (ii)). So we can define the height $h_{\bar{L}}(Z)$ of a cycle $Z$ on $X$ by

$$
h_{\bar{L}}(Z)=h_{\overline{L^{\prime}}}\left(f_{*}(Z)\right)
$$

To make this definition meaningful, we have to show that $h_{\overline{L^{\prime}}}\left(f_{*}(Z)\right)$ does not depend on the choice of $X^{\prime}, \overline{L^{\prime}}$, and $f$. Then it is easily seen that Proposition 3.2.1 still holds in the general case.

Let, for $i=1,2, \bar{L}^{\prime}$ be an hermitian line bundle over a smooth projective arithmetic variety $X_{i}^{\prime}$ and $f_{i}: X \rightarrow X_{i}^{\prime}$ an $S$-morphism such that $f_{i}^{*} \bar{L}_{i}^{\prime} \simeq \bar{L}$. We must show that, for any $Z \in Z_{p}(X)$,

$$
h_{\overline{L_{1}^{\prime}}}\left(f_{1 *}(Z)\right)=h_{\overline{L_{2}^{\prime}}}\left(f_{2 *}(Z)\right)
$$

Let $X^{\prime}:=X_{1}^{\prime} \times_{S} X_{2}^{\prime}, f:=\left(f_{1}, f_{2}\right): X \rightarrow X^{\prime}, p r_{i}: X_{1}^{\prime} \times_{S} X_{2}^{\prime} \rightarrow X_{i}^{\prime}$ be the projections, and $\bar{M}_{i}=p r_{i}^{*} \bar{L}_{i}^{\prime}$.

Using (3.2.1), we get: 


$$
\begin{aligned}
h_{\overline{L^{\prime}}}( & \left(f_{2 *}(Z)\right)-h_{{\overline{L^{\prime}}}_{1}}\left(f_{1 *}(Z)\right)=h_{\bar{M}_{2}}\left(f_{*}(Z)\right)-h_{\bar{M}_{1}}\left(f_{*}(Z)\right) \\
& =\widehat{\operatorname{deg}}\left(\widehat{c}_{1}\left(\bar{M}_{2}\right)^{p} \mid f_{*}(Z)\right)-\widehat{\operatorname{deg}}\left(\widehat{c}_{1}\left(\bar{M}_{1}\right)^{p} \mid f_{*}(Z)\right) \\
& =\sum_{i+j=p-1} \widehat{\operatorname{deg}}\left(\widehat{c}_{1}\left(\bar{M}_{1}\right)^{i} \widehat{c}_{1}\left(\bar{M}_{2}\right)^{j} \widehat{c}_{1}\left(\bar{M}_{2} \otimes \bar{M}_{1}^{\vee}\right) \mid f_{*}(Z)\right) .
\end{aligned}
$$

The isomorphisms of hermitian line bundles

$$
f^{*} \bar{M}_{1} \simeq f_{1}^{*}{\overline{L^{\prime}}}_{1} \simeq \bar{L} \simeq f_{2}^{*}{\overline{L^{\prime}}}_{2} \simeq f^{*} \bar{M}_{2}
$$

show that there exists a nonvanishing regular section of $\left(M_{2} \otimes M_{1}^{\vee}\right)_{\mid f(|Z|)}$ of norm 1 on $f(|Z|)(\mathbb{C})$. Therefore, by (2.3.8), for any $x \in \widehat{C H}^{p-1}\left(X^{\prime}\right)$, $\widehat{\operatorname{deg}}\left(x \widehat{c}_{1}\left(\bar{M}_{2} \otimes \bar{M}_{1}\right) \mid f_{*}(Z)\right)=0$. This proves the required equality.

\subsubsection{Dependence on metrics and integral structures.}

Proposition 3.2.2. Let $\bar{L}=(L, h)$ be an hermitian line bundle on a projective arithmetic variety $X$, and let $M_{K}$ be an ample line bundle on $X_{K}$.

(i) Assume that $h^{\prime}=e^{\varphi} h$ is another hermitian scalar product on $L$. Then, for any cycle $Z \in Z_{p}(X)$ :

$$
h_{(L, h)}(Z)-h_{\left(L, h^{\prime}\right)}(Z)=\frac{1}{2} \sum_{i+j=p-1} \int_{X(\mathbb{C})} \varphi c_{1}(L, h)^{i} c_{1}\left(L, h^{\prime}\right)^{j} \delta_{Z}
$$

In particular, when the smooth function $\varphi$ is constant,

$$
h_{(L, h)}(Z)-h_{\left(L, h^{\prime}\right)}(Z)=\frac{p}{2} \varphi[K: \mathbb{Q}] \operatorname{deg}_{L_{K}}(Z) .
$$

Moreover, there exists $C \in \mathbb{R}_{+}$, depending only on $L_{\mathbb{C}}, h, h^{\prime}$, and $M_{K}$, such that, if $Z$ is effective,

$$
\left|h_{(L, h)}(Z)-h_{\left(L, h^{\prime}\right)}(Z)\right| \leq C \operatorname{deg}_{M_{K}} Z_{K}
$$

(ii) For any choice of a projective arithmetic variety $X^{\prime}$ and of an hermitian line bundle $\overline{L^{\prime}}$ on $X^{\prime}$ such that $\left(X_{K}^{\prime}, L_{K}^{\prime}\right)$ is isomorphic to $\left(X_{K}, L_{K}\right)$ (i.e., such that there exists an isomorphism of schemes $f: X_{K} \simeq X_{K}^{\prime}$ for which $f^{*} L_{K}^{\prime} \simeq$ $L_{K}$ ), there exists $C \in \mathbb{R}_{+}$such that, for any effective cycle $Z_{K}$ on $X_{K}$,

$$
\left|h_{\overline{L^{\prime}}}\left(f_{*}\left(Z_{K}\right)\right)-h_{\bar{L}}\left(Z_{K}\right)\right| \leq C \operatorname{deg}_{M_{K}} Z_{K} .
$$

Observe that the inequalities (3.2.5) and (3.2.6) do not really depend on the choice of the ample line bundle $M_{K}$ since, for any two ample line bundles $M_{K}$ and $M_{K}^{\prime}$ on $X_{K}$, there exist $C_{1}$ and $C_{2}$ in $\mathbb{R}_{+}^{*}$ such that, for any effective cycle $Z_{K}$ on $X_{K}$, the following inequalities hold: 


$$
C_{1} \operatorname{deg}_{M_{K}} Z_{K} \leq \operatorname{deg}_{M_{K}^{\prime}} Z_{K} \leq C_{2} \operatorname{deg}_{M_{K}} Z_{K} \text {. }
$$

Proof. Using the construction of 3.2.1, Remark, one easily sees that to prove (i), one may assume $X$ regular. Note that, by the definition of $\widehat{c}_{1}$ in 2.1.2,

$$
\widehat{c}_{1}(L, h)-\widehat{c}_{1}\left(L, h^{\prime}\right)=a(\varphi) .
$$

Together with (2.1.2), this implies:

$$
\widehat{c}_{1}(L, h)^{p}-\widehat{c}_{1}\left(L, h^{\prime}\right)^{p}=a\left(\varphi \sum_{i+j=p-1} c_{1}(L, h)^{i} c_{1}\left(L, h^{\prime}\right)^{j}\right),
$$

where $c_{1}(L, h)=\omega\left(\widehat{c}_{1}(L, h)\right)$ is the first Chern form of $\left(L_{\mathbb{C}}, h\right)$. Formula

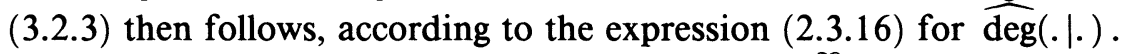

To prove (3.2.5), observe that there exists a $C^{\infty}$ hermitian metric on the ample line bundle $M_{K}$ whose first Chern form $\alpha$ is strictly positive on $X(\mathbb{C})$. According to Proposition 1.1.4, (iv), there exists $C \in \mathbb{R}_{+}$such that

$$
-C . \alpha^{p-1} \leq \frac{1}{2} \sum_{i+j=p-1} c_{1}(L, h)^{i} c_{1}\left(L, h^{\prime}\right)^{j} \leq C . \alpha^{p-1} .
$$

Then (3.2.5) follows from (3.2.3), since

$$
\int_{X(\mathbb{C})} \alpha^{p-1} \delta_{Z}=[K: \mathbb{Q}] \operatorname{deg}_{M_{K}}\left(Z_{K}\right)
$$

Under the hypotheses of (ii), the closure in $X{ }^{\prime} X^{\prime}$ of the graph of $f$ is a projective arithmetic variety $\widetilde{X}$, and the two projections from $\widetilde{X}$ to $X$ and $X^{\prime}$ are isomorphisms over $\operatorname{Spec}(K)$. Therefore, to prove (ii), we may assume that the isomorphism $f: X_{K} \simeq X_{K}^{\prime}$ extends to a morphism $f: X \rightarrow X^{\prime}$. Moreover, it follows from inequalities (3.2.7) that the line bundle $M_{K}$ over $X_{K}$ may be assumed to be the restriction of an ample line bundle $M$ over $X$. Finally, using (3.2.5), we may assume that the isomorphism $f^{*} L_{K}^{\prime} \simeq L_{K}$ is isometric. Then this isomorphism defines a rational section $\sigma$ of the hermitian line bundle $f^{*} \overline{L^{\prime}} \otimes \bar{L}^{-1}$ over $X$ such that $\|\sigma\|=1$ and the $\operatorname{divisor} \operatorname{div}(\sigma)$ is supported by closed fibers of $X$.

Let $Z_{K}$ be any effective cycle in $Z_{p-1}\left(X_{K}\right)$, and let $Z \in Z_{p}(X)$ be its Zariski clusure. Then $f_{*}(Z)$ is the Zariski closure of $f_{*}\left(Z_{K}\right)$. Therefore

$$
h_{\bar{L}}\left(Z_{K}\right)=h_{\bar{L}}(Z) \text { and } h_{\overline{L^{\prime}}}\left(f_{*}\left(Z_{K}\right)\right)=h_{\overline{L^{\prime}}}\left(f_{*}(Z)\right) \text {. }
$$

Applying Proposition 3.2.1, (iii), we now get:

$$
\begin{aligned}
h_{\overline{L^{\prime}}} & \left(f_{*}\left(Z_{K}\right)\right)-h_{\bar{L}}\left(Z_{K}\right)=h_{f^{*}\left(\bar{L}^{\prime}\right)}(Z)-h_{\bar{L}}(Z) \\
& =\widehat{\operatorname{deg}}\left(\widehat{c}_{1}\left(f^{*}\left(\overline{L^{\prime}}\right)\right)^{p}-\widehat{c}_{1}(\bar{L})^{p} \mid Z\right) \\
& =\sum_{i+j=p-1} \widehat{\operatorname{deg}}\left(\widehat{c}_{1}\left(f^{*}\left(\overline{L^{\prime}}\right)\right)^{i} \widehat{c}_{1}(\bar{L})^{j} \widehat{c}_{1}\left(f^{*}{\overline{L^{\prime}}}^{\prime} \otimes \bar{L}^{-1}\right) \mid Z\right) .
\end{aligned}
$$


According to Proposition 2.3.1, (vi) and 2.3.1, Remark (ii) (applied to each component $Y$ of $Z$, which meets $\operatorname{div}(\sigma)$ properly), we have

$$
\begin{gathered}
\widehat{\operatorname{deg}}\left(\widehat{c}_{1}\left(f^{*}\left(\overline{L^{\prime}}\right)\right)^{i} \widehat{c}_{1}(\bar{L})^{j} \widehat{c}_{1}\left(f^{*} \overline{L^{\prime}} \otimes \bar{L}^{-1}\right) \mid Z\right) \\
=\widehat{\operatorname{deg}}\left(\widehat{c}_{1}\left(f^{*}\left(\overline{L^{\prime}}\right)\right)^{i} \widehat{c}_{1}(\bar{L})^{j} \mid \operatorname{div}(\sigma) . Z\right) .
\end{gathered}
$$

The intersection cycle $\operatorname{div}(\sigma) . Z$ is supported on some closed fibers of $X$, and we shall estimate the right-hand side of (3.2.9) by applying in these closed fibers the following standard result:

Lemma 3.2.3. Let $V$ be a projective scheme over a field $k$, let $\mathscr{M}$ be an ample line bundle on $V$, and let

$$
\operatorname{deg}_{k}: C H_{0}(V) \rightarrow \mathbb{Z}
$$

be the composition $\mathrm{CH}_{0}(V) \stackrel{p_{*}}{\rightarrow} \mathrm{CH}_{0}(\operatorname{Spec}(k)) \simeq \mathbb{Z}$, where $p$ denotes the structural morphism $V \rightarrow \operatorname{Spec}(k)$. For any finite family $\left(\mathscr{L}_{1}, \ldots, \mathscr{L}_{n}\right)$ of line bundles over $V$, there exists $C \in \mathbb{R}_{+}$such that the following equality holds for any effective cycle $T \in Z_{n}(V)$ :

$$
\left|\operatorname{deg}_{k}\left(c_{1}\left(\mathscr{L}_{1}\right) \cdots c_{1}\left(\mathscr{L}_{n}\right) \cdot[T]\right)\right| \leq C \operatorname{deg}_{k}\left(c_{1}(\mathscr{M})^{n} \cdot[T]\right) .
$$

Indeed, we can write

$$
\operatorname{div}(\sigma)=\sum_{\wp \in I} D_{\wp},
$$

where $I$ is a finite set of nonzero prime ideals in $\mathscr{C}_{K}$, and where $D_{\wp}$ is a Cartier divisor supported on $X_{\mathrm{F}_{p}}$. We have

$$
\operatorname{div}(\sigma) \cdot Z=\sum_{\wp \in I} D_{\wp} \cdot Z
$$

and the cycle $D_{\wp} . Z$ may be seen as a cycle on $X_{\mathrm{F}_{p}}$, and, by the same argument as in 3.1.2.2, we have

$$
\begin{aligned}
& \widehat{\operatorname{deg}}\left(c_{1}\left(f^{*}\left(\overline{L^{\prime}}\right)\right)^{i} \widehat{c}_{1}(\bar{L})^{j} \mid D_{\wp} \cdot Z\right) \\
& =\operatorname{deg}_{\mathbb{F}_{\mathfrak{p}}}\left(c_{1}\left(f^{*}\left(L^{\prime}\right)_{\mathbb{F}_{\mathfrak{p}}}\right)^{i} c_{1}\left(L_{\mathbb{F}_{\mathfrak{p}}}\right)^{j} \cap\left[D_{\wp} . Z\right]\right) \log N \wp .
\end{aligned}
$$

For any $\wp \in I$, we can find $k \in \mathbb{N}$ such that the Cartier divisors $k X_{\mathbb{F}_{p}}+D_{\wp}$ and $k X_{\mathbb{F}_{p}}-D_{\wp}$ are effective. Then the cycles $\left(k X_{\mathbb{F}_{p}}+D_{\wp}\right) \cdot Z$ and $\left(k X_{\mathbb{F}_{p}}-D_{\wp}\right) \cdot Z$ are effective, and Lemma 3.2.3, applied to $V=X_{\mathrm{F}_{\mathrm{p}}}, \mathscr{M}=M_{\mathrm{F}_{\mathrm{p}}}, n=p-1$, $\mathscr{L}_{1}=\cdots=\mathscr{L}_{i}=f^{*}\left(L^{\prime}\right)_{\mathrm{F}_{p}}, \mathscr{L}_{i+1}=\cdots=\mathscr{L}_{p-1}=L_{\mathrm{F}_{\mathrm{p}}}^{\prime}$, and $T=\left(k X_{\mathrm{F}_{p}}+D_{\wp}\right) \cdot Z$ or $\left(k X_{\mathrm{F}_{p}}-D_{\wp}\right) \cdot Z$, shows the existence of a constant $C(i, j, \wp)$ such that, for any flat effective $Z \in Z_{p}(X)$, 


$$
\begin{aligned}
& \left|\operatorname{deg}_{\mathbb{F}_{p}}\left(c_{1}\left(f^{*}\left(L^{\prime}\right)_{\mathbb{F}_{p}}\right)^{i} c_{1}\left(L_{\mathbb{F}_{p}}\right)^{j} \cdot\left[D_{\wp} \cdot Z\right]\right)\right| \\
& \quad \leq C(i, j, \wp) \operatorname{deg}_{\mathbb{F}_{p}}\left(c_{1}\left(M_{\mathbb{F}_{p}}\right)^{p-1}\left[X_{\mathbb{F}_{p}} \cdot Z\right]\right) .
\end{aligned}
$$

The compatibility of intersection operations and specialization ([Fu2], 20.3 and Example 20.3.3) shows that, for any $\wp$ :

$$
\operatorname{deg}_{\mathbb{F}_{p}}\left(c_{1}\left(M_{\mathbb{F}_{p}}\right)^{p-1}\left[X_{\mathbb{F}_{p}} \cdot Z\right]\right)=\operatorname{deg}_{M_{K}} Z_{K}
$$

Finally, we get from (3.2.8)-(3.2.12):

$$
\left|h_{\overline{L^{\prime}}}\left(f\left(Z_{K}\right)\right)-h_{\bar{L}}\left(Z_{K}\right)\right| \leq \sum_{\substack{i+j=p-1 \\ \boldsymbol{p} \in I}} C(i, j, \wp) \operatorname{deg}_{M_{K}} Z_{K}
$$

Remark. Let $V$ be a smooth projective variety over $\overline{\mathbb{Q}}$, and let $L, M$ be two line bundles on $V$, with $M$ ample. Let $K$ be a number field such that $V$ and $L$ can be defined over $K$, let $\mathscr{V}$ be a projective arithmetic variety over $\operatorname{Spec}\left(\mathscr{O}_{K}\right)$, and let $\overline{\mathscr{L}}$ be an hermitian line bundle over $\mathscr{V}$ such that $\mathscr{V} \overline{\mathbb{Q}} \simeq V$ and $\mathscr{L}_{\overline{\mathbb{Q}}} \simeq L$. It follows from Proposition 3.2.2 that the function on the set of nonzero effective cycles on $X_{\overline{\mathbb{Q}}}$ which sends a cycle $Z$ to

$$
\left(\operatorname{deg}_{M}(Z)\right)^{-1} \cdot h_{\overline{\mathscr{L}}}(Z) \in \mathbb{R}
$$

does not depend on the choices of $K, \mathscr{V}$, and $\overline{\mathscr{L}}$, up to an error term which is uniformly bounded when $Z$ varies. See also [Gu], $\S 6$ and $\S 7$, for similar considerations.

3.2.3. Positivity. The following statement extends Proposition 2.6 in [Fa2] to the heights $h_{\bar{L}}$.

Proposition 3.2.4. Let $\bar{L}$ be an hermitian line bundle on a projective arithmetic variety $X$. Assume that $c_{1}(\bar{L})$ is positive and that some positive power $L^{n}$ of $L$ is generated by global sections of sup norm less than or equal to one. Then, for any effective cycle $Z$ on $X$,

$$
h_{\bar{L}}(Z) \geq 0
$$

Proof. We prove this by induction on the dimension of $Z$. When $\operatorname{dim}(Z)=$ 0 this follows from (3.1.4). When $\operatorname{dim}(Z)>0$, we may assume that $Z$ is irreducible and we can choose a rational section of $L^{n}$ of sup norm $\leq 1$ which does not vanish identically on $Z$ (otherwise the fiber of $L^{n}$ at the generic point of $Z$ would not be generated by global sections of $L^{n}$ ). Calling $s$ its restriction to $Z$ we deduce from (3.2.2) that

$$
n h_{\bar{L}}(Z) \geq h_{\bar{L}}(\operatorname{div}(s))
$$

and the result follows since $\operatorname{dim}(\operatorname{div}(s))=\operatorname{dim}(Z)-1$. 
Remarks. (i) Let us use the notations of 3.1.2.3. If $\bar{E}$ is the trivial hermitian vector bundle of rank $N+1$ (i.e., the direct sum of $N+1$ copies of $\overline{\mathscr{O}}=$ $(\mathscr{O},\|\|),\|1\|=1)$, the hermitian line bundle $\overline{\mathscr{O}(1)}$ on $\mathbb{P}(E)$ satisfies the hypotheses of Proposition 3.2.4. Therefore, we recover Proposition 2.6 in [Fa2], which asserts that $h_{F}(Z) \geq 0$ for any effective cycle $Z$ on $\mathbb{P}(E)$ (see Proposition 4.1.3 and Theorem 5.2.3 for a better estimate).

(ii) The relation between the positivity of the height $h_{\bar{L}}$ and the existence of "small" sections for some power of $L^{n}$ has been thoroughly studied by Zhang in $[\mathrm{Zh} 1],[\mathrm{Zh} 2]$.

(iii) It follows from (3.2.4) and Proposition 3.2.4 that if we only assume that $c_{1}(\bar{L})$ is positive and that some positive power $L^{n}$ is generated by its global sections over $X$, then there exists a constant $C \in \mathbb{R}_{+}$such that, for any effective cycle $Z$ on $X$,

$$
h_{\bar{L}}(Z) \geq-C \operatorname{deg}_{L_{K}}(Z) .
$$

Using Proposition 3.2.2, we also get that, if some positive power $L_{K}^{n}$ is generated by its global sections over $X_{K}$, and if $M_{K}$ is an ample line bundle over $X_{K}$, there exists $C \in \mathbb{R}_{+}$such that for any effective cycle $Z$ on $X_{K}$

$$
h_{\bar{L}}(Z) \geq-C \operatorname{deg}_{M_{K}}(Z) \text {. }
$$

(iv) Proposition 3.2.4 and the preceding remark extend to multiheights. For instance, if $\bar{L}_{1}, \ldots, \bar{L}_{p}$ are hermitian line bundles on a projective arithmetic variety such that $c_{1}\left(\bar{L}_{1}\right), \ldots, c_{1}\left(\bar{L}_{p}\right)$ are positive and some positive powers $L_{1}^{n_{1}}, \ldots, L_{p}^{n_{p}}$ are generated by global sections, then there exist constants $C_{1}, \cdots, C_{p} \in \mathbb{R}_{+}$such that for any effective cycle $Z \in Z_{p}(X)$

$$
\widehat{\operatorname{deg}}\left(\widehat{c}_{1}\left(\bar{L}_{1}\right) \cdots \widehat{c}_{1}\left(\bar{L}_{p}\right) \mid Z\right) \geq-\sum_{i=1}^{p} C_{i} \operatorname{deg}_{K}\left(\prod_{\substack{j=1 \\ j \neq i}}^{p} c_{1}\left(L_{j}\right) \mid Z\right) .
$$

\subsubsection{Finiteness.}

Theorem 3.2.5. Let $\bar{L}$ be an hermitian line bundle on a projective arithmetic variety $X$. If $L$ is ample on $X$, then for any real number $A>0$, there exists only finitely many effective cycles $Z \in Z_{p}(X)$ such that $\operatorname{deg}_{L_{K}}(Z) \leq A$ and $h_{\bar{L}}(Z) \leq A$.

Proof. First, by Proposition 3.2.1, (i), we may replace $L$ by a positive power, and therefore assume that $L$ is very ample. Then there exists a closed immersion $i: X \rightarrow \mathbb{P}_{S}^{N}=\mathbb{P}\left(\mathscr{O}_{S}^{\oplus(N+1)}\right)$ such that $L \simeq i^{*} \mathscr{O}(1)$. We shall equip $\mathscr{O}(1)$ with the hermitian metric defined by the trivial hermitian metric on $\mathscr{\sigma}_{S}^{\oplus(N+1)}$; then Proposition 3.2.2, (i) shows that we may assume that $\bar{L}=i^{*} \overline{\mathscr{O}(1)}$, and Proposition 3.2.1, (iii) reduces the proof of Theorem 3.2.5 to the case where $X=\mathbb{P}_{S}^{N}$ and $\bar{L}=\overline{\mathscr{O}(1)}$.

Any effective cycle $Z \in Z_{p}\left(\mathbb{P}_{S}^{N}\right)$ may be decomposed as 


$$
Z=Z_{h}+\sum_{\wp} Z_{\wp}
$$

where $Z_{h}$ is effective and flat over $S$, where $\wp$ runs over the nonzero prime ideals of $\mathscr{O}_{K}$, and where $Z_{\wp}$ is an effective cycle on $\mathbb{P}_{\mathbb{F}_{\wp}}^{N}$ which vanishes for almost every $\wp$. Let us denote by $\operatorname{deg}_{\mathbb{F}_{p}} Y$ the degree of a cycle $Y \in Z_{p}\left(\mathbb{P}_{\mathbb{F}_{p}}^{N}\right)$ (i.e., with the same notation as in Lemma 3.2.3, $\left.\operatorname{deg}_{\mathbb{F}_{p}} Y=\operatorname{deg}_{\mathbb{F}_{\mathfrak{p}}}\left(c_{1}(\mathscr{O}(1))^{p} \cdot[Y]\right)\right)$. The positivity of the height $h_{F}=h_{\bar{L}}$ (3.2.3, Remark (i)) and formula (3.1.3) show that

$$
h_{F}\left(Z_{h}\right) \leq h_{F}(Z)
$$

and

$$
\operatorname{deg}_{\mathbb{F}_{\wp}} Z_{\wp} \cdot \log N_{\wp} \leq h(Z) .
$$

Since $Z_{\emptyset}$ is effective, $\operatorname{deg}_{\mathbb{F}_{\emptyset}} Z_{\emptyset} \geq 1$ if $Z_{\wp} \neq 0$. Therefore (3.2.15) implies that, if $h(Z) \leq A$, we have $Z_{\wp}=0$ unless

$$
\log N \wp \leq A .
$$

There is only a finite set of $\wp$ satisfying (3.2.16). Moreover, for any given $\wp$, the set of effective cycles on $\mathbb{P}_{\mathbb{F}_{\wp}}^{N}$ of degree less that $(\log N \wp)^{-1} . A$ is finite (this follows for instance from the classical theory of Chow forms, cf. [C-Wa], [Sa], I.9). Therefore there is only a finite set of possibilities for the "vertical parts" $\sum_{\wp} Z_{\wp}$ in the decomposition (3.2.13) of the effective cycles $Z$ such that $h(Z) \leq A$.

Together with (3.2.14), this shows that, to prove Theorem 3.2.5, it is enough to prove that a set of flat effective cycles of bounded degrees and heights is finite. As hinted in [So2], this follows from the computation of the height of Chow forms (see 4.3.4 below). One can also deduce it from the previous statements in this section together with the following geometrical fact (which is another consequence of the classical theory of Chow forms):

Lemma 3.2.6. For any $p \in\{1, \ldots, N\}$ and any integer $D$, there exists a finite set $H_{1}, \ldots, H_{M}$ of hyperplanes in $\mathbb{P}_{K}^{N}$ which satisfies the following condition: for any two distinct effective cycles $Z_{K}$ and $Z_{K}^{\prime}$ in $Z_{p}\left(\mathbb{P}_{K}^{N}\right)$ of degrees at most $D$, there exists $i \in\{1, \ldots, M\}$ such that $H_{i}$ meets $Z_{K}$ and $Z_{K}^{\prime}$ properly and the cycles $H_{i} . Z_{K}$ and $H_{i} . Z_{K}^{\prime}$ are distinct.

Indeed, let $p \in\{1, \ldots, N\}$, and let $Z \in Z_{p+1}\left(\mathbb{P}_{S}^{N}\right)$ be a flat effective cycle on $X$ whose height and degree are bounded by $A$. We apply Lemma 3.2.6 with some $D \geq A$. For any $i \in\{1, \ldots, M\}$, let $s_{i}$ be a section of $\mathscr{O}(1)$ over $\mathbb{P}_{S}^{N}$ such that $\mathscr{H}_{i}:=\operatorname{div}\left(s_{i}\right)$ restricts to $H_{i}$ on $\mathbb{P}_{K}^{N}$. If $H_{i}$ meets $Z$ properly, 
then $\mathscr{H}_{i}$ meets $Z$ properly, and $\mathscr{H}_{i} . Z$ is an effective cycle in $Z_{p}\left(\mathbb{P}_{S}^{N}\right)$ which restricts to $H_{i} . Z_{K}$ in the generic fiber, therefore

$$
h_{F}\left(H_{i} \cdot Z_{K}\right) \leq h_{F}\left(\mathscr{H}_{i} \cdot Z\right)
$$

Moreover, we get from (3.2.2):

$$
h_{F}\left(\mathscr{H}_{i} \cdot Z\right) \leq h_{F}(Z)+[K: \mathbb{Q}] c_{i} \operatorname{deg}_{\mathscr{O}(1)_{K}}(Z) \leq A\left(1+[K: \mathbb{Q}] c_{i}\right)
$$

where $c_{i}$ is the sup norm of $\log \left\|s_{i}\right\|$. Furthermore

$$
\operatorname{deg}_{\mathscr{O}(1)_{K}}\left(H_{i} \cdot Z_{K}\right)=\operatorname{deg}_{\mathscr{O}(1)_{K}}\left(Z_{K}\right) \leq A .
$$

Lemma 3.2.6 together with (3.2.17) and (3.2.18) reduces the proof of Theorem 3.2.5 for flat cycles of relative dimension $p \in\{1, \ldots, N\}$ in $\mathbb{P}_{S}^{N}$ to the proof for flat cycles of relative dimension $p-1$. As it is true when $p=0$ by the classical theory of heights ([No], [We2]) Theorem 3.2.5 follows.

If we combine Theorem 3.2.5 with Proposition 3.2.2, (ii) and formulae (3.1.9) and (3.1.10), we get:

Corollary 3.2.7. Let $\bar{L}$ be an hermitian line bundle on a projective arithmetic variety $X$. If $L_{K}$ is ample on $X_{K}$, then for any real number $A>0$, there exist only finitely many effective cycles $Z \in Z\left(X_{\overline{\mathbb{Q}}}\right)$ defined over a number field $K^{\prime} \supset$ $K$ of degree $\left[K^{\prime}: K\right] \leq A$ and such that $\operatorname{deg}_{L_{\overline{\mathbf{Q}}}}(Z) \leq A$ and $h_{\bar{L}, \text { norm }}(Z) \leq A$.

3.2.5. Hilbert-Samuel formula. Let $\bar{L}$ be an hermitian line bundle over a projective arithmetic variety $X$, and $Z$ an integral subscheme of dimension $p$ in $X$, flat over $S$. When $L_{K}$ is ample, the degree $\operatorname{deg}_{L_{K}}\left(Z_{K}\right)$ is given by the leading term of the Hilbert polynomial of $L_{K \mid Z_{K}}$. Namely, we have the following "Hilbert-Samuel formula", as $n$ goes to infinity:

$$
\operatorname{dim}_{K} H^{0}\left(Z_{K} ; L_{K}^{n}\right)=\operatorname{deg}_{L_{K}}\left(Z_{K}\right) \frac{n^{p-1}}{(p-1) !}+O\left(n^{p-2}\right)
$$

We now want to describe an arithmetic counterpart to this formula, which again illustrates the analogy between the degree of varieties over a field and the height of arithmetic varieties.

Let $H^{0}\left(Z, L^{n}\right)$ be the set of sections of $L^{n}$ over $Z, n \geq 0$. This is a torsion free $\mathbb{Z}$-module. We endow it with the sup norm $\|\cdot\|_{\infty}$ of sections of $L^{n}$ over $X(\mathbb{C})$. Let $V_{\infty}$ be the covolume of $H^{0}\left(Z, L^{n}\right)$ in $H^{0}\left(Z, L^{n}\right) \underset{\mathbb{Z}}{\otimes} \mathbb{R}$ for the Haar measure which gives volume one to the unit ball, and $\widehat{\operatorname{deg}}\left(H^{0}\left(Z, L^{n}\right),\|\cdot\|_{\infty}\right)$ $=-\log \left(V_{\infty}\right)$.

Theorem 3.2.8. Assume that $L$ is ample and that $c_{1}(\bar{L})$ is positive. As $n$ goes to infinity, the following asymptotic formula holds: 


$$
\widehat{\operatorname{deg}}\left(H^{0}\left(Z, L^{n}\right),\|\cdot\|_{\infty}\right)=h_{\bar{L}}(Z) \frac{n^{p}}{p !}+O\left(n^{p-1} \log n\right) .
$$

This formula is shown in [G-S4], [B2], 4.3, and [G-S6], 5.1.2 when $Z_{K}$ is smooth and $c_{1}(\bar{L})$ is strictly positive, and by Zhang [Zh2] in general.

Observe that when $X=\mathbb{P}_{\mathbb{Z}}^{N}$ and $\bar{L}=\overline{\mathscr{O}(1)}$, Theorem 3.2 .8 provides a very concrete interpretation of the Faltings height of an irreducible subscheme $Z \subset$ $\mathbb{P}_{\mathbb{Z}}^{N}$ in terms of the homogeneous ideal $I \subset \mathbb{Z}\left[X_{0}, \ldots, X_{N}\right]$ which defines it: indeed, for $n$ large enough, the left-hand side of (3.2.19) is then the covolume of $\mathbb{Z}\left[X_{0}, \ldots, X_{N}\right]_{n} / I_{n}$ equipped with the norm \|\|$_{\infty}$ defined by

$$
\|[P]\|_{\infty}=\sup _{\left(z_{0}: \ldots: z_{N}\right) \in Z(\mathbb{C})} \frac{\left|P\left(z_{0}, \ldots, z_{N}\right)\right|}{\left(\sum_{i=0}^{N}\left|z_{i}\right|^{2}\right)^{n / 2}}
$$

\subsection{More examples.}

3.3.1. Faltings heights of hypersurfaces and of projective spaces. Let $\bar{E}$ be an hermitian vector bundle of rank $N+1$ over $S, \mathbb{P}(E)$ the projective bundle of $E, \overline{\mathscr{O}(1)}$ the standard hermitian line bundle on $\mathbb{P}(E)$, and $s$ a global section of $\mathscr{O}(d)$ on $\mathbb{P}(E)$. For any complex imbedding $\sigma: K \rightarrow \mathbb{C}$, we may identify $\mathbb{P}(E)_{\sigma}(\mathbb{C})$ with $\mathbb{P}^{N}(\mathbb{C})$ by choosing an orthonormal basis for $E_{\sigma}$. The section $s$ then defines an homogeneous polynomial $P_{\sigma} \in \mathbb{C}\left[X_{0}, \ldots, X_{N}\right]$ of degree $d$. Let $D=\operatorname{div}(s)$, and let $d v$ be the $U(N+1)$-invariant probability measure on the unit sphere $\mathbb{S}^{2 N+1}$ in $\mathbb{C}^{N+1}$.

Applying formula (3.2.2) to $Z=\mathbb{P}(E)$ and $\bar{L}=\overline{\mathscr{O}(1)}$ and then formula (1.4.7), we find:

$$
\begin{aligned}
h_{F}(D) & =d h_{F}(\mathbb{P}(E))+\int_{\mathbb{P}(E)(\mathbb{C})} \log \|s\| c_{1}(\overline{\mathscr{O}(1)})^{N} \\
& =d h_{F}(\mathbb{P}(E))+\sum_{\sigma: K \rightarrow \mathbb{C}} \int_{\mathbb{S}^{2 N+1}} \log \left|P_{\sigma}(x)\right| d v
\end{aligned}
$$

Assume now that $\bar{E}=\overline{\mathscr{O}}_{S}^{N+1}$ is trivial and let $\mathbb{P}_{S}^{N}=\mathbb{P}\left(\mathscr{O}_{S}^{N+1}\right)$. Using the preceding formula for the height of hypersurfaces, we can easily compute the Faltings height of $\mathbb{P}_{S}^{N}$ in terms of the Stoll number $\sigma_{N}$ defined in (1.4.3).

Lemma 3.3.1. The Faltings height of $\mathbb{P}_{S}^{N}$ is

$$
h_{F}\left(\mathbb{P}_{S}^{N}\right)=[K: \mathbb{Q}] \sigma_{N}
$$

Proof (see also [G-S3], p. 212). Let $s$ be the section of $\mathscr{O}(1)$ defined by the homogeneous coordinate $X_{0}$; then $D=\mathbb{P}_{S}^{N-1}$. Applying (3.3.1) and the identity (see 1.4.3, Remark (iii))

$$
\int_{\mathrm{S}^{2 N+1}} \log \left|X_{0}\right| d v=-\frac{1}{2} \sum_{m=1}^{N} \frac{1}{m}
$$


we get

$$
\begin{aligned}
h_{F}\left(\mathbb{P}_{S}^{N-1}\right) & =h_{F}\left(\mathbb{P}_{S}^{N}\right)+[K: \mathbb{Q}] \int_{\mathbb{S}^{2 N+1}} \log \left|X_{0}\right| d v \\
& =h_{F}\left(\mathbb{P}_{S}^{N}\right)-[K: \mathbb{Q}] \frac{1}{2}\left(\sum_{m=1}^{N} \frac{1}{m}\right) .
\end{aligned}
$$

Since $h_{F}\left(\mathbb{P}_{S}^{0}\right)=0$, the result follows by induction on $N$.

3.3.2. Heights of linear projections. Let $\bar{E}$ be an hermitian vector bundle on $S$, let $F$ be a subbundle of $E$, and let $\overline{E / F}$ be the quotient bundle, equipped with the quotient metric. Let $Z$ be an effective cycle in $Z_{p}\left(\mathbb{P}(E)_{K}\right)$, no component of which is contained in $\mathbb{P}(F)_{K}$. We shall compare the Faltings height of $Z$, defined by means of the hermitian vector bundle $\bar{E}$, with the Faltings height of its linear projection $p_{*}(Z)$ in $\mathbb{P}(E / F)_{K}$, defined by means of the hermitian vector bundle $\overline{E / F}$. This will extend Proposition 2.10 in [Fa2].

First recall how $p_{*}(Z)$ is defined. Let $\nu: B \rightarrow \mathbb{P}(E)$ be the blow-up of $\mathbb{P}(E)$ along $\mathbb{P}(F)$ and $D \subset B$ the exceptional divisor. Denote by $Q$ the canonical quotient bundle on $\mathbb{P}(E / F)$, and by $p r_{1}$ (resp. $p r_{2}$ ) the projection of $\mathbb{P}(E) \times_{S} \mathbb{P}(E / F)$ onto the first (resp. second) factor. The projective arithmetic variety $B$ may be identified with the subscheme of $\mathbb{P}(E) \times_{S} \mathbb{P}(E / F)$ defined by the vanishing of the regular section $\sigma$ of $p r_{1}^{*} \mathscr{O}_{E}(1) \otimes p r_{2}^{*} Q$ attached to the composite of the following tautological maps:

$$
\begin{aligned}
p r_{1}^{*} \mathscr{O}_{E}(-1) \rightarrow p r_{1}^{*} \pi_{\mathbb{P}(E)}^{*} E & \simeq \pi_{\mathbb{P}(E) \times_{S} \mathbb{P}(E / F)}^{*} E \rightarrow \pi_{\mathbb{P}(E) \times_{s} \mathbb{P}(E / F)}^{*} E / F \\
& \simeq p r_{2}^{*} \pi_{\mathbb{P}(E / F)}^{*} E / F \rightarrow p r_{2}^{*} Q .
\end{aligned}
$$

Then the restriction of $p r_{1}$ to $B$ is equal to $\nu$, and the restriction of $p r_{2}$ to $B$ is a smooth morphism such that, on $\mathbb{P}(E)-\mathbb{P}(F), f \circ \nu^{-1}$ coincides with the linear projection onto $\mathbb{P}(E / F)$. The cycle $p_{*}(Z) \in Z_{p}\left(\mathbb{P}(E / F)_{K}\right)$ is defined as the image $f_{*} \widetilde{Z}$ of the proper transform $\widetilde{Z}$ of $Z$ in $B_{K}$.

By definition of Faltings heights,

$$
h_{F}(Z)=h_{F}(\bar{Z})=\widehat{\operatorname{deg}}\left(\widehat{c}_{1}\left(\overline{\mathscr{O}_{E}(1)}\right)^{p+1} \mid \bar{Z}\right),
$$

where $\bar{Z}$ is the Zariski closure of $Z$, and

$$
h_{F}\left(p_{*}(Z)\right)=h_{F}\left(\overline{p_{*}(Z)}\right)=\widehat{\operatorname{deg}}\left(\widehat{c}_{1}\left(\overline{\mathscr{G}_{E / F}(1)}\right)^{p+1} \mid \overline{p_{*}(Z)}\right) \text {. }
$$

On the other hand, we have the equalities of cycles

$$
\bar{Z}=\nu_{*}(\overline{\widetilde{Z}})
$$

and

$$
\overline{p_{*}(Z)}=f_{*}(\overline{\widetilde{Z}})
$$


Therefore, by (2.3.6), we get:

$$
h_{F}(Z)=\widehat{\operatorname{deg}}\left(\nu^{*} \widehat{c}_{1}\left(\overline{\mathscr{O}_{E}(1)}\right)^{p+1} \mid \overline{\widetilde{Z}}\right)
$$

and

$$
h_{F}\left(p_{*}(Z)\right)=\widehat{\operatorname{deg}}\left(f^{*} \widehat{c}_{1}\left(\overline{\mathscr{O}_{E / F}(1)}\right)^{p+1} \mid \overline{\widetilde{Z}}\right)
$$

Consider the hermitian line bundle

$$
\bar{L}:=\nu^{*} \overline{\mathscr{O}(1)} \otimes f^{*} \overline{\mathscr{O}_{E / F}(-1)}
$$

over $B$. According to the definition of $B$ by the vanishing of $\sigma$, the canonical map

$$
p r_{1}^{*} \mathscr{O}_{E}(-1) \rightarrow p r_{2}^{*} \pi_{\mathbb{P}(E / F)}^{*} E / F
$$

introduced above takes values in the subbundle $\operatorname{pr}_{2}^{*} \mathscr{O}_{E / F}(-1)$ when restricted to $B$, hence defines a regular section $s$ of $L$ over $B$, which is easily seen to be $\neq 0$, to have $D$ as divisor, and to have norm $\leq 1$. Using (3.3.3), (3.3.4), and Proposition 2.3.1, (vi), we get:

$$
\begin{aligned}
& h_{F}(Z)-h_{F}\left(p_{*}(Z)\right)=\widehat{\operatorname{deg}}\left(\nu^{*} \widehat{c}_{1}\left(\overline{\mathscr{O}_{E}(1)}\right)^{p+1}-f^{*} \widehat{c}_{1}\left(\overline{\mathscr{O}_{E / F}(1)}\right)^{p+1} \mid \overline{\widetilde{Z}}\right) \\
& =\widehat{\operatorname{deg}}\left(\widehat{c}_{1}(\bar{L}) \cdot \sum_{i+j=p} \nu^{*} \widehat{c}_{1}\left(\overline{\mathscr{O}_{E}(1)}\right)^{i} \cdot f^{*} \widehat{c}_{1}\left(\overline{\mathscr{\sigma}_{E / F}(1)}\right)^{j} \mid \overline{\widetilde{Z}}\right) \\
& \widehat{=\operatorname{deg}}\left(\sum_{i+j=p} \nu^{*} \widehat{c}_{1}\left(\overline{\mathscr{O}_{E}(1)}\right)^{i} \cdot f^{*} \widehat{c}_{1}\left(\overline{\mathscr{\mathscr { O }}_{E / F}(1)}\right)^{j} \mid D \cdot \overrightarrow{\widetilde{Z}}\right) \\
& +\int_{B(\mathbb{C})}\left(\sum_{i+j=p} \nu^{*} c_{1}\left(\overline{\mathscr{O}_{E}(1)}\right)^{i} \cdot f^{*} c_{1}\left(\overline{\mathscr{O}_{E / F}(1)}\right)^{j}\right) \cdot \log \|s\|^{-1} \cdot \delta_{\widetilde{Z}}
\end{aligned}
$$

As this last integral is nonnegative (since $c_{1}\left(\overline{\mathscr{O}_{E}(1)}\right)$ and $c_{1}\left(\overline{\mathscr{G}_{E / F}(1)}\right)$ are positive $(1,1)$ forms, $\|s\| \leq 1$, and $\tilde{Z}$ is effective) the difference $h_{F}(Z)-$ $h_{F}\left(p_{*}(Z)\right)$ is bounded below by the sum of the real numbers

$$
\widehat{\operatorname{deg}}\left(\nu^{*} \widehat{c}_{1}\left(\overline{\mathscr{O}_{E}(1)}\right)^{i} \cdot f^{*} \widehat{c}_{1}\left(\overline{\mathscr{O}_{E / F}(1)}\right)^{j} \mid D . \overline{\widetilde{Z}}\right), i+j=p,
$$

which are nothing else than the biheights of the effective cycle $D . \bar{Z}$ on $D=\mathbb{P}(F) \times_{S} \mathbb{P}(E / F)$ attached to the hermitian line bundles $\nu^{*} \overline{\mathscr{O}_{E}(1)}$ and $f^{*} \overline{\mathscr{O}_{E / F}(1)}$. It then follows from 3.2.3, Remark (iii) that $h_{F}(Z)-h_{F}\left(p_{*}(Z)\right)$ 
is bounded below by a linear expression in the bidegrees of D. $\tilde{Z}$ in $\mathbb{P}(F)_{K} \times_{K}$ $\mathbb{P}(E / F)_{K}$, whose coefficients depend only on $\bar{E}$ and $F$.

In particular, when $|Z|_{K}$ does not meet $\mathbb{P}(F)_{K}$, the cycle $D . \overline{\widetilde{Z}}$ is effective and supported on closed fibers of $B$, so we get the inequality:

$$
h_{F}(Z) \geq h_{F}\left(p_{*}(Z)\right) \text {. }
$$

In other words, the height of an effective cycle decreases by linear projection from a center that it does not meet in the generic fiber.

Suppose moreover that $Z$ is irreducible and that $F$ is a line bundle. Then $\mathbb{P}(F)$ defines a point $P \in P(E)(S)$, the support of the subscheme $P \cap \bar{Z}$ is a finite set of closed points $\left\{Q_{1}, \ldots, Q_{n}\right\}$, and the Segre class of $P \cap \bar{Z}$ in $\bar{Z}$ takes the form

$$
s(P \cap \bar{Z}, \bar{Z})=\sum_{i=1}^{n} m_{i} Q_{i},
$$

where $m_{i}$ is the multiplicity of $\bar{Z}$ along $P \cap \bar{Z}$ at $Q_{i}$ (cf. [Fu2], $\S \S 4.2$ and 4.3). In that case, the expression (3.3.6) vanishes if $i \geq 1$, and, for $i=0$, is easily seen to be

$$
\sum_{i=1}^{n} m_{i} \log \# k\left(Q_{i}\right) .
$$

Therefore, $h_{F}(Z)-h_{F}\left(p_{*}(Z)\right)$ is the sum of this weighted sum of multiplicities and of the integral in (3.3.5), which may be interpreted as the "archimedean multiplicity of $Z$ at $P \cap Z$ ".

3.3.3. The Arakelov-Faltings invariant $\left(\bar{\omega}_{X / S} \cdot \bar{\omega}_{X / S}\right)$. We now indicate how invariants of arithmetic surfaces introduced by Arakelov and Faltings fit into our framework.

Suppose that $X$ is a semi-stable regular arithmetic surface over $S$ (i.e., $X$ is a regular projective arithmetic variety of dimension $2, X_{F}$ is geometrically irreducible of genus $\geq 1$, and the closed fibers of $\pi: X \rightarrow S$ are semi-stable curves). When $\bar{L}$ is the hermitian line bundle $\bar{\omega}_{X / S}$ defined as the relative dualizing sheaf $\omega_{X / S}$ equipped with the Arakelov metric \|\|$_{A}$ at infinite places (cf. [Ar1], [Fa1]), the height $h_{\bar{L}}(X)$ is the real number $\left(\bar{\omega}_{X / S} \cdot \bar{\omega}_{X / S}\right)$ attached to $X$ considered by Arakelov [Ar2] and Faltings [Fa1].

More generally, if $X^{\prime}$ is any semi-stable model of $X_{K}$ over $S$ (not necessarily regular), the relative dualizing sheaf $\omega_{X^{\prime} / S}$ is still a well-defined line bundle on $X^{\prime}$, which may be equipped with the Arakelov metric \|\|$_{A}$, and we can consider the height $h_{\bar{\omega}_{X^{\prime} / S}}\left(X^{\prime}\right)$ defined by $\bar{\omega}_{X^{\prime} / S}=\left(\omega_{X^{\prime} / S},\|\|_{A}\right)$. This height does not in fact depend on the semi-stable model $X^{\prime}$ of $X_{K}$. Indeed, there exists a unique morphism $f: X \rightarrow X^{\prime}$ which extends the identity on the generic fiber ( $f$ is the contraction of some rational curves of self-intersection -2 in the closed fibers of $X$ ) and the identification 


$$
\omega_{X / S \mid X_{K}} \simeq \omega_{X_{K}} \simeq\left(f^{*} \omega_{X^{\prime} / S}\right)_{\mid X_{K}}
$$

extends to an isomorphism of hermitian line bundles

$$
\bar{\omega}_{X / S} \simeq f^{*} \bar{\omega}_{X^{\prime} / S}
$$

Therefore, we get from (3.2.1) and 3.2.1, Remark:

$$
h_{\bar{\omega}_{X / S}}(X)=h_{\bar{\omega}_{X^{\prime} / S}}\left(f_{*}(X)\right)=h_{\bar{\omega}_{X^{\prime} / S}}\left(X^{\prime}\right),
$$

since $f$ is birational.

In particular, we can take as $X^{\prime}$ the stable model of $X_{K}$. As the formation of this stable model and of its relative dualizing sheaf $\omega_{X^{\prime} / S}$ is compatible with the base changes $S^{\prime}=\operatorname{Spec}\left(\mathscr{O}_{K^{\prime}}\right) \rightarrow S=\operatorname{Spec}\left(\mathscr{O}_{K}\right)$ defined by extensions $K \hookrightarrow K^{\prime}$ of number fields, we recover from 3.1.4 the fact that the real number

$$
e(X)=[K: \mathbb{Q}]^{-1} h_{\bar{\omega}_{X / S}}(X)
$$

is an invariant of $X_{\bar{\Phi}}$. This fact, as well as (3.3.8), was originally due to MoretBailly ([MB3], 3.3 and 5.4; the coincidence of $h_{\bar{\omega}_{X^{\prime} / S}}\left(X^{\prime}\right)$ and of $\left\langle\bar{\omega}_{X^{\prime} / S}, \bar{\omega}_{X^{\prime} / S}\right\rangle$ as defined by Moret-Bailly, using Deligne's pairing, follows from (2.3.11), (3.2.2), and [MB2], 6.7-8; see also [G-S3], Theorem 4.10.1, (i) when $X^{\prime}$ is smooth over $S$ ).

3.3.4. Heights on abelian varieties. Let $A$ be an abelian variety over $K$, and $L$ be a line bundle on $A$. Given any nonempty subset $I \subset\{1,2,3\}$, let

$$
p_{I}: A^{3} \rightarrow A
$$

be the morphism sending a geometric point $\left(x_{1}, x_{2}, x_{3}\right)$ to $\sum_{j \in I} x_{j}$, where $A^{3}$ is the product over $K$ of three copies of $A$. According to the theorem of the cube, the line bundle

$$
\begin{aligned}
C(L):= & \underset{\substack{I \subset\{1,2,3\} \\
I \neq \varnothing}}{\otimes} p_{I}^{*}(L)^{(-1)^{* I}} \\
= & p_{1}^{*}(L)^{-1} \otimes p_{2}^{*}(L)^{-1} \otimes p_{3}^{*}(L)^{-1} \\
& \otimes p_{12}^{*}(L) \otimes p_{23}^{*}(L) \otimes p_{13}^{*}(L) \otimes p_{123}^{*}(L)^{-1}
\end{aligned}
$$

may be trivialized on $A^{3}$. The choice of an isomorphism

$$
c: C(L) \stackrel{\sim}{\rightarrow} \mathscr{O}_{A^{3}}
$$

is equivalent to the choice of the nonzero element $c^{-1}(1)(0)$ in the fiber of $C(L)$ at the origin of $A^{3}$, i.e., to the choice of a nonzero element in the fiber of $L^{-1}$ at the origin of $A$.

To simplify the discussion, assume that $A$ has good reduction over $S$, i.e., there exists an abelian scheme $\mathscr{A}$ over $S$ with generic fiber $A$. The projections 
$p_{I}$ extend in the obvious way to morphisms from $\mathscr{A}^{3}:=\mathscr{A} \times_{S} \mathscr{A} \times{ }_{S} \mathscr{A}$ to $\mathscr{A}$, and for any hermitian line bundle $\overline{\mathscr{L}}$ on $\mathscr{A}$ we define $C(\overline{\mathscr{L}})$ as an hermitian line bundle on $\mathscr{A}^{3}$ by the same formula as (3.3.9). According to Faltings [FaW], II.2, and Moret-Bailly [MB 1$]$, Chapter II, given any pair $(L, c)$ of a line bundle $L$ with an isomorphism $c: C(L) \stackrel{\sim}{\rightarrow} \mathscr{O}_{A^{3}}$, there exists a unique (up to unique isomorphism) pair $(\overline{\mathscr{L}}, \bar{c})$ formed by an hermitian line bundle $\overline{\mathscr{L}}$ on $\mathscr{A}$ and an isometric isomorphism

$$
\bar{c}: C(\overline{\mathscr{L}}) \stackrel{\sim}{\rightarrow} \overline{\mathscr{O}}_{\mathscr{A}^{3}}
$$

where $\overline{\mathscr{O}}_{\mathscr{A}^{3}}$ denotes $\mathscr{O}_{\mathscr{A}^{3}}$ equipped with the trivial metric (defined by $\|1\|=$ 1 ), such that $\left.\mathscr{L}\right|_{A}=L$ and $\left.\bar{c}\right|_{A^{3}}=c$. Moreover, the class of $\overline{\mathscr{L}}$ in $\widehat{\operatorname{Pic}}(\mathscr{A})$ depends only on the class of $L$ in $\operatorname{Pic}(A)$.

The height $h_{\bar{L}}$ will then be denoted $h_{L \text {, cub }}$. The isometric isomorphism $\bar{c}$ implies that given three points $P_{1}, P_{2}, P_{3}$ in $A(K)$ the following holds:

$$
\sum_{\substack{I \subset\{1,2,3\} \\ I \neq \varnothing}}(-1)^{\# I} h_{L, \text { cub }}\left(\sum_{j \in I} P_{j}\right)=0 .
$$

Therefore, on rational points, the height $h_{L \text {, cub }}$ coincides with the Néron-Tate height defined by $L$; see [Fa-W], II.2, and [MB1], Chapter III.

For any integer $n \in \mathbb{Z}$ let $[n]$ be the endomorphism of $A$ or $\mathscr{A}$ mapping $x$ to $n x$. Assume that $L$ is symmetric, i.e., $[-1]^{*} L \simeq L$. Then we get $[-1]^{*} \widehat{c}_{1}(\overline{\mathscr{L}})=\widehat{c}_{1}(\overline{\mathscr{L}})$ and, by the cubical property $(3.3 .10)$ of $\overline{\mathscr{L}},[n]^{*} \widehat{c}_{1}(\overline{\mathscr{L}})=$ $n^{2} \widehat{c}_{1}(\overline{\mathscr{L}})$. Therefore, given any cycle $\mathscr{Z} \in Z_{p}(\mathscr{A})$, we have

$$
h_{L, \mathrm{cub}}\left([n]_{*} \mathscr{Z}\right)=n^{2 p} h_{L, \mathrm{cub}}(\mathscr{Z})
$$

and

$$
\operatorname{deg}_{L}\left([n]_{*} \mathscr{Z}\right)=n^{2 p-2} \operatorname{deg}_{L}(\mathscr{Z}) .
$$

If, in addition, $L$ is very ample and such that $A$ is projectively normal when imbedded using sections of $L$, Philippon defines a height $h_{P}(Z)$ for any effective cycle $Z$ on $A$ (cf. [P2], Proposition 9, p. 281; note however that in [loc.cit.] $A$ need not have good reduction over $S$ ). Philippon's height is characterized by the following two properties:

(i) for any effective cycle $Z$ on $A$ of dimension $p-1$ and for any $n \in$ $\mathbb{N}-\{0\}$,

$$
h_{P}\left([n]_{*} Z\right)=n^{2 p} h_{P}(Z) \text {; }
$$

(ii) there exists a constant $C \in \mathbb{R}_{+}$such that, for any effective cycle $Z$ on $A$,

$$
\left|h_{P}(Z)-h_{\bar{L}}(Z)\right| \leq C \operatorname{deg}_{L}(Z)
$$


(in [P2] one considers instead of $h_{\bar{L}}$ the height of the Chow form of $Z$ for the projective imbedding given by sections of $L$, but these are known to be equivalent; see 4.3 .4 below).

When $A$ has good reduction, these properties are satisfied by $h_{L \text {, cub }}$ according to (3.3.11) and Proposition 3.2.2(ii); therefore Philippon's height $h_{P}$ coincides with the cubical height $h_{L \text {, cub }}$ on effective cycles on $A$. The equality of cubical and Philippon's heights allows one to recover various properties of the latter. For instance, let $P \in A(K)$ be a torsion point of order $n$ and let $t: \mathscr{A} \rightarrow \mathscr{A}$ be the translation by $P$ (considered by properness as an element of $\left.\mathscr{A}\left(\mathscr{O}_{K}\right)\right)$. The cubical property of $\overline{\mathscr{L}}$ implies then that

$$
n^{2} \cdot t^{*} \widehat{c}_{1}(\overline{\mathscr{L}})=n^{2} \cdot \widehat{c}_{1}(\overline{\mathscr{L}})
$$

therefore $h_{L, \text { cub }}(Z)=h_{L \text {, cub }}\left(t_{*}(Z)\right)$ for any cycle $Z$ on $A$, as is also shown in [P2].

Finally, we may also describe $h_{L \text {, cub }}$ using arithmetic fundamental classes as follows. Let $s$ be a nontrivial rational section of $\mathscr{L}$ on $\mathscr{A}$ and $\Theta$ its divisor. Recall that, for any complex embedding $\sigma$ of $K, A_{\sigma}$ denotes the complex abelian variety deduced from $A$ by the extension of scalars $\sigma: K \rightarrow \mathbb{C}$. Let $\mu \in A^{1,1}\left(A_{\mathbb{R}}\right)$ be a Kähler form whose restriction to each component $A_{\sigma}(\mathbb{C})$ is translation invariant. Then the $\mu$-harmonic forms on $A(\mathbb{C})$ are the forms whose restriction to each component $A_{\sigma}(\mathbb{C})$ is translation invariant, and the harmonic projection $H: \mathscr{D}^{00}(A(\mathbb{C})) \rightarrow \mathscr{H}^{00}(A(\mathbb{C}))$ sends $u \in \mathscr{D}^{00}(A(\mathbb{C}))$ to the function which takes as constant value $H_{\sigma}(u)$ on $A_{\sigma}(\mathbb{C})$ the integral of $u_{\mid A_{\sigma}(\mathbb{C})}$ with respect to the Haar measure of volume 1 on $A_{\sigma}(\mathbb{C})$. The cubical condition (3.3.10) on $\overline{\mathscr{L}}$ implies that $c_{1}(\overline{\mathscr{L}})$ is translation irvariant on every component $A_{\sigma}(\mathbb{C})$ (cf. [Fa-W], II.2, and [MB2], II.2). Therefore

$$
d d^{c}\left(-\log \|s\|^{2}\right)+\delta_{\Theta} \in \mathscr{H}^{1,1}\left(A_{\mathbb{R}}\right)
$$

and the $\mu$-normalized Green current for $\Theta$ is

$$
g_{\boldsymbol{\Theta}}=-\log \|s\|^{2}+H\left(\log \|s\|^{2}\right)
$$

Therefore

$$
\begin{aligned}
\widehat{c}_{1}(\overline{\mathscr{L}}) & =\left[\left(\Theta,-\log \|s\|^{2}\right)\right] \\
& =\left[\left(\Theta, g_{\Theta}\right)\right]-a\left(H\left(\log \|s\|^{2}\right)\right) \\
& =[\Theta]_{\mu}-a\left(H\left(\log \|s\|^{2}\right)\right)
\end{aligned}
$$

and, using (2.3.20), we get the following formula for any $\mathscr{Z} \in Z_{p}(\mathscr{A})$ :

$$
h_{L, \text { cub }}(\mathscr{Z})=\widehat{\operatorname{deg}}\left([\mathscr{Z}]_{\mu} \cdot[\Theta]_{\mu}^{p}\right)-p \operatorname{deg}_{K}\left(\mathscr{Z}_{K} \cdot \Theta_{K}^{p-1}\right) \sum_{\sigma: K \rightarrow \mathbb{C}} H_{\sigma}(\log \|s\|) .
$$


The possibility of such an expression for Philippon's height was indicated in [Hi]. For other approaches to the height of cycles on abelian varieties, see [Gu] and $[\mathrm{Kr}]$.

\section{THE HEIGHT OF CYCLES IN PROJECTIVE SPACES}

\subsection{The projective height.}

4.1.1. Definition. Let $\bar{E}$ be an hermitian vector bundle of rank $N+1$ over $S, N \geq 0$. The canonical quotient bundle $Q$ on $\mathbb{P}(E)$ is defined by the exact sequence

$$
\mathscr{E}: 0 \rightarrow \mathscr{O}(-1) \rightarrow \pi^{*} E \rightarrow Q \rightarrow 0
$$

on $\mathbb{P}(E)$, where $\pi: \mathbb{P}(E) \rightarrow S$ is the projection map. It will be equipped with the quotient metric deduced from $\pi^{*} \bar{E}$, while $\mathscr{O}(-1)$ will be equipped with the induced metric, which coincides with the dual of the metric on $\mathscr{O}(1)$ introduced in 3.1.2.3. For any $p \geq 0$ we let $\widehat{c}_{p}(\bar{Q}) \in \widehat{C H}^{p}(\mathbb{P}(E))$ be the $p$-th arithmetic Chern class of $\bar{Q}$ (see 2.1.2).

Definition 4.1.1. For any cycle $Z \in Z_{p}(\mathbb{P}(E))$ we let

$$
h(Z)=\widehat{\operatorname{deg}}\left(\widehat{c}_{p}(\bar{Q}) \mid Z\right) \in \mathbb{R},
$$

and for any $Z \in Z_{p-1}\left(\mathbb{P}(E)_{K}\right)$, we let

$$
h(Z)=h(\bar{Z})
$$

where $\bar{Z} \in Z_{p}(\mathbb{P}(E))$ is the Zariski closure of $Z$.

The real number $h(Z)$ will be called the projective height of the cycle $Z$ defined by the hermitian vector bundle $\bar{E}$. When we shall need to make the dependence on $\bar{E}$ explicit, we shall write $h^{\bar{E}}(Z)$ instead of $h(Z)$.

When $p \geq 1$, we shall also write $\operatorname{deg}_{K}(Z)$ for the usual degree of $Z$, namely

$$
\operatorname{deg}_{K}(Z)=\operatorname{deg}_{\mathcal{O}(1)_{K}}\left(Z_{K}\right)=\operatorname{deg}_{K}\left(c_{p-1}\left(Q_{K}\right) \mid Z_{K}\right)=\operatorname{deg}_{K}\left(\widehat{c}_{p-1}(\bar{Q}) \mid Z\right) \in \mathbb{Z}
$$

4.1.2. First properties and examples. The next proposition first states that the number $h(Z)$ defined by (4.1.1) coincides with Faltings' height $h_{F}(Z):=$ $h_{\overline{\mathscr{O}(1)}}(Z)$ (cf. 3.1.2.3) up to some additive normalization. In particular, $h(Z)$ deserves to be called a height. As shown by the second part of the next proposition, with this normalization the height of linear subspaces of $\mathbb{P}(E)$ takes a simple form which, contrary to that of Faltings height, does not involve the Stoll numbers $\sigma_{n}$.

Proposition 4.1.2. (i) For any cycle $Z \in Z_{p}(\mathbb{P}(E))$, we have if $p \geq 1$ :

$$
h(Z)=h_{F}(Z)+\left(\widehat{\operatorname{deg}}(\bar{E})-[K: \mathbb{Q}] \sigma_{p-1}\right) \operatorname{deg}_{K}(Z) ;
$$

$$
\text { if } p=0 \text { and } Z=\sum_{i} n_{i} P_{i} \text {, where } n_{i} \in \mathbb{Z} \text { and } P_{i} \text { are closed points of } X \text {, }
$$




$$
h(Z)=h_{F}(Z)=\sum_{i} n_{i} \log \# k\left(P_{i}\right) .
$$

(ii) Let $\bar{F} \subset \bar{E}$ be any nonzero subbundle of $E$, equipped with the induced metric. Then

$$
h^{\bar{E}}(\mathbb{P}(F))=\widehat{\operatorname{deg}}(\bar{E})-\widehat{\operatorname{deg}}(\bar{F}) .
$$

If $r$ denotes the rank of $F, h^{\bar{E}}(\mathbb{P}(F))-\widehat{\operatorname{deg}}(\bar{E})$ coincides with the Faltings height of the point $\mathbb{P}\left(\Lambda^{r} F\right)$ in $\mathbb{P}\left(\Lambda^{r} E\right)$ defined by the hermitian vector bundle $\Lambda^{r} \bar{E} .^{4}$

In particular, when $\bar{E}$ is trivial, $h(\mathbb{P}(F))$ is the classical height (cf. (3.1.6)) of the Plücker point of $F_{K}$ in $\mathbb{P}^{M}(K), M=\left(\begin{array}{c}N+1 \\ r\end{array}\right)-1$. Thus the height of $\mathbb{P}(F)$ coincides with the (logarithm of the) height introduced by Schmidt [Sch].

Observe that (4.1.2) and (4.1.3) provide the following generalization of Lemma 3.3.1:

$$
h_{F}(\mathbb{P}(F))=-\widehat{\operatorname{deg}}(\bar{F})+[K: \mathbb{Q}] \sigma_{r-1} .
$$

Proof. To prove (i) we need to compare $c_{p}(\bar{Q})$ with $\widehat{c}_{1}(\overline{\mathscr{O}(1)})^{p}$ since, by definition, $h_{F}(Z)=h_{\overline{\mathscr{O}}(1)}(Z)($ see $(3.1 .5))$.

Identity (2.1.4) applied to the canonical exact sequence $\mathscr{E}$ gives

$$
\widehat{c}\left(\pi^{*} \bar{E}\right)=\left(1-\widehat{c}_{1}(\overline{\mathscr{O}(1)})\right) \cdot \widehat{c}(\bar{Q})-a(\widetilde{c}(\mathscr{E})) .
$$

Indeed $\widehat{c}_{1}(\overline{\mathscr{O}(-1)})=-\widehat{c}_{1}(\overline{\mathscr{O}(1)})$ and $\widehat{c}_{p}(\overline{\mathscr{O}(-1)})=0$ if $p \geq 2$, since $\mathscr{O}(-1)$ has rank 1 . The computation of the Bott-Chern class $\widetilde{c_{p}}(\mathscr{E})$ is purely archimedean, so that we may identify $\bar{E}_{\mathbb{C}}$ and $\mathbb{C}^{N+1}$ with the standard metric. According to [G-S3], Proposition 5.3, if we write $\mu=c_{1}(\overline{\mathscr{O}(1)})$, we have:

$$
\tilde{c}_{0}(\mathscr{E})=0 \text { and } \tilde{c_{k}}(\mathscr{E})=-\left(\sum_{m=1}^{k-1} \frac{1}{m}\right) \mu^{k-1} \text { if } k \geq 1 .
$$

Using (4.1.5) and (2.1.2) we get, when $p \geq 1$,

$$
\widehat{c}_{p}(\bar{Q})=\widehat{c}_{1}\left(\overline{\mathscr{O}}(1)^{p}+\widehat{c}_{1}\left(\overline{\mathscr{O}(1)}^{p-1}\right) \pi^{*} \widehat{c}_{1}(\bar{E})+a\left(\sum_{i \geq 0} \mu^{i} \widetilde{c}_{p-i}(\mathscr{E})\right),\right.
$$

hence, by (1.4.3) and (4.1.6),

$$
\widehat{c}_{p}(\bar{Q})=\widehat{c}_{1}\left(\overline{\mathscr{O}(1)}^{p}\right)+\widehat{c}_{1}\left(\overline{\mathscr{O}(1)}^{p-1}\right) \pi^{*} \widehat{c}_{1}(\bar{E})-2 a\left(\sigma_{p-1} \mu^{p-1}\right) .
$$

\footnotetext{
${ }^{4}$ For any embedding $\sigma: K \rightarrow \mathbb{C}$, the hermitian scalar product $\langle,\rangle_{\Lambda^{r}}$ on $\Lambda^{r} E_{\sigma}$ is defined in terms of the hermitian scalar product $\langle$,$\rangle on E_{\sigma}$ by $\left\langle v_{1} \wedge \cdots \wedge v_{r}, w_{1} \wedge \cdots \wedge w_{r}\right\rangle_{\Lambda^{r}}=$ $\operatorname{det}\left(\left\langle v_{i}, w_{j}\right\rangle\right)_{1 \leq i, j \leq r}$.
} 
Formula (4.1.2) now follows from the definitions of $h_{F}(Z)$ and $h(Z)$, since (2.3.20) gives the identity

$$
\widehat{\operatorname{deg}}\left(\widehat{c}_{1}(\overline{\mathscr{O}(1)})^{p-1} \cdot \pi^{*} \widehat{c}_{1}(\bar{E}) \mid Z\right)=\operatorname{deg}_{K}\left(\widehat{c}_{1}(\overline{\mathscr{O}(1)})^{p-1} \mid Z\right) \widehat{\operatorname{deg}} \widehat{c}_{1}(\bar{E}),
$$

while

$$
\operatorname{deg}_{K}\left(\widehat{c}_{1}(\overline{\mathscr{O}(1)})^{p-1} \mid Z\right)=\operatorname{deg}_{K}(Z)
$$

and

$$
\widehat{\operatorname{deg}}\left(2 a\left(\sigma_{p-1} \mu^{p-1}\right) \mid Z\right)=[K: \mathbb{Q}] \operatorname{deg}_{K}(Z) \sigma_{p-1} \text {. }
$$

When $p=0, h(Z)$ and $h_{F}(Z)$ are both equal to $\widehat{\operatorname{deg}}(1 \mid Z)$, and $\left(4.1 .2^{\prime}\right)$ follows from (2.3.16).

(ii) Let $i: \mathbb{P}(F) \rightarrow \mathbb{P}(E)$ be the inclusion, $\pi_{F}: \mathbb{P}(F) \rightarrow S$ be the projection, and $Q_{F}$ be the canonical quotient bundle on $\mathbb{P}(F)$. On $\mathbb{P}(F)$ there is a canonical exact sequence

$$
0 \rightarrow Q_{F} \rightarrow i^{*} Q \rightarrow \pi^{*}(E / F) \rightarrow 0 .
$$

Let us equip the bundles in (4.1.8) with the metric induced from $\pi^{*} \bar{E}$. Then (4.1.8), as an exact sequence of hermitian holomorphic vector bundles, is split over the complex points of $\mathbb{P}(F):$ for any imbedding $\sigma: K \rightarrow \mathbb{C}, i^{*}\left(\bar{Q}_{\sigma}\right)$ is the orthogonal direct sum of $\left(\bar{Q}_{F}\right)_{\sigma}$ with $\pi_{\sigma}^{*}\left(\bar{F}_{\sigma}^{\perp}\right)$, where $\bar{F}_{\sigma}^{\perp}$ is the orthogonal complement to $\bar{F}_{\sigma}$ in $\bar{E}_{\sigma}$. It follows that the Bott-Chern classes of (4.1.8) vanish and, by (2.1.5),

$$
i^{*} \widehat{c}(\bar{Q})=\widehat{c}\left(\bar{Q}_{F}\right) \cdot \pi_{F}^{*} \widehat{c}(\overline{E / F}) .
$$

Since $Q_{F}$ has rank $r-1, \widehat{c}_{r}\left(\bar{Q}_{F}\right)=0$. Furthermore

$$
\widehat{c}_{1}(\overline{E / F})=\widehat{c}_{1}(\bar{E})-\widehat{c}_{1}(\bar{F})
$$

since the metrics are induced from $\bar{E}$. Therefore

$$
i^{*} \widehat{c}_{r}(\bar{Q})=\widehat{c}_{r-1}\left(\bar{Q}_{F}\right) \cdot \pi_{F}^{*}\left(\widehat{c}_{1}(\bar{E})-\widehat{c}_{1}(\bar{F})\right) .
$$

Using (2.3.6) and (2.3.20) we get

$$
\begin{aligned}
h(\mathbb{P}(F)) & =\widehat{\operatorname{deg}}\left(\widehat{c}_{r}(\bar{Q}) \mid \mathbb{P}(F)\right) \\
& =\widehat{\operatorname{deg}}\left(\widehat{c}_{r-1}\left(\bar{Q}_{F}\right) \cdot \pi_{F}^{*}\left(\widehat{c}_{1}(\bar{E})-\widehat{c}_{1}(\bar{F})\right) \mid \mathbb{P}(F)\right) \\
& =\operatorname{deg}_{K}\left(c_{r-1}\left(Q_{F}\right) \mid \mathbb{P}(F)\right) \cdot \widehat{\operatorname{deg}}\left(\widehat{c}_{1}(\bar{E})-\widehat{c}_{1}(\bar{F})\right) .
\end{aligned}
$$

Since

$$
\operatorname{deg}_{K}\left(c_{r-1}\left(Q_{F}\right) \mid \mathbb{P}(F)\right)=1,
$$

formula (4.1.3) follows. 
Let $\varepsilon: S \rightarrow \mathbb{P}\left(\Lambda^{r} E\right)$ be the section of $\pi: \mathbb{P}\left(\Lambda^{r} E\right) \rightarrow S$ defined by the subbundle $\Lambda^{r} F$ of $\Lambda^{r} E$. We have a canonical isometric isomorphism

$$
\varepsilon^{*} \overline{\mathscr{O}_{\Lambda^{r} E}(1)} \simeq{\overline{\Lambda^{r} F}}^{\vee}
$$

Therefore

$$
h_{\overline{\mathscr{O}^{r} E}(1)}(\mathbb{P}(F))=\widehat{\operatorname{deg}} \varepsilon^{*} \overline{\mathscr{O}_{\Lambda^{r} E}(1)}=-\widehat{\operatorname{deg}} \overline{\Lambda^{r} F}=-\widehat{\operatorname{deg}} \bar{F} .
$$

According to (4.1.3), this equals $h^{\bar{E}}(\mathbb{P}(F))-\widehat{\operatorname{deg}} \bar{E}$.

As will be demonstrated in the sequel, many formulas involving the height of cycles in projective spaces are simplified by the use of the normalized height $h$. Moreover, it enjoys nice positivity properties, which strengthen 3.2.3, Remark (i). They will be established in 5.2 below. However, we can already prove:

Proposition 4.1.3. Assume that $\bar{E}$ is trivial and that, on $\mathbb{P}_{K}^{N}$, the cycle $Z \in$ $Z_{p}(\mathbb{P}(E))$ meets properly (i.e., does not meet) one of the linear subspaces of codimension $p$ defined by the vanishing of $p$ of the canonical homogeneous coordinates $\left(X_{0}, \ldots, X_{N}\right)$. Then, if $Z$ is effective,

$$
h(Z) \geq 0 \text {. }
$$

Proof. Let $\mathbb{P}^{N-p} \subset \mathbb{P}^{N}$ be a linear subspace of codimension $p$ defined by the vanishing of $p$ homogeneous coordinates, and assume $Z \in Z_{p}\left(\mathbb{P}^{N}\right)$ does not meet $\mathbb{P}^{N-p}$ on $\mathbb{P}_{K}^{N}$. Let $\Lambda$ be the Levine form of $\mathbb{P}^{N-p}$ (see 1.2.3, Example $(\mathrm{v}))$. From [G-S3], Theorem 5.2, we know that the class of $\left(\mathbb{P}^{N-p}, \Lambda\right)$ in $\widehat{C H}^{p}\left(\mathbb{P}^{N}\right)$ is equal to $\widehat{c}_{p}(\bar{Q})$. On the other hand, if $Z$ is effective, the class $\left[\mathbb{P}^{N-p}\right][Z]$ in $C H_{\mathbb{P}^{N-p} \cap|Z|}^{N+1}\left(\mathbb{P}^{N}\right)$ can be represented by an effective cycle $\sum_{\alpha} m_{\alpha} W_{\alpha}$ ([Fu2], 12.2, 20.1, and 20.2). From (2.3.16) it follows that

$$
h(Z)=\sum_{\alpha} m_{\alpha} \log \# k\left(W_{\alpha}\right)+\frac{1}{2} \int_{\mathbb{P}^{N}(\mathbb{C})} \Lambda d_{Z} .
$$
tive.

This is nonnegative since $\Lambda \geq 0$ (Proposition 1.4.1, (i)) and $Z$ is effec-

\subsection{The height of joins.}

4.2.1. Arithmetic integral geometry. A very useful tool in the study of heights is provided by the behavior of degrees under algebraic correspondences. Namely, let us consider a diagram

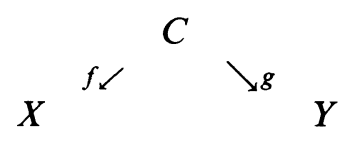

where $X, Y, C$ are regular projective arithmetic varieties, $f$ is flat, and $f_{K}$ is smooth. Given any cycle $Z$ on $X$, we define its image under the correspondence $C$ to be the cycle 


$$
C_{*}(Z)=g_{*} f^{*}(Z)
$$

on $Y$. Given a class $y \in \widehat{C H}^{*}(Y)$ we let

$$
C^{*}(y)=f_{*} g^{*}(y) \in \widehat{C H}^{*}(X) .
$$

Lemma 4.2.1. Under these hypotheses, the equality

$$
\left(y \mid C_{*}(Z)\right)=\left(C^{*}(y) \mid Z\right)
$$

holds in $\widehat{C H}^{*}(S)_{\mathbb{Q}}$.

Proof. We apply Proposition 2.3.1, (iv) to $g$ and Proposition 2.3.1, (v) to $f$ to get

$$
\begin{aligned}
\left(y \mid C_{*}(Z)\right) & =\left(y \mid g_{*} f^{*}(Z)\right)=\left(g^{*}(y) \mid f^{*}(Z)\right) \\
& =\left(f_{*} g^{*}(y) \mid Z\right)=\left(C^{*}(y) \mid Z\right) .
\end{aligned}
$$

To compute $C^{*}(y)$, a useful remark is the following. Assume that, for any embedding $\sigma: K \hookrightarrow \mathbb{C}$, there exists a compact Lie group $G_{\sigma}$ acting upon $X_{\sigma}(\mathbb{C}), Y_{\sigma}(\mathbb{C}), C_{\sigma}(\mathbb{C})$ in such a way that $f_{\sigma}$ and $g_{\sigma}$ are equivariant maps and that $X_{\sigma}(\mathbb{C})$ is an hermitian symmetric quotient of $G_{\sigma}$. Then, if $\omega(y)_{\mid Y_{\sigma}(\mathbb{C})}$ is invariant under the action of $G_{\sigma}$, the same will be true for $\omega\left(C^{*}(y)\right)_{\mid Y_{\sigma}(\mathrm{C})}$, hence this class will be harmonic for any $G_{\sigma}$-invariant Kähler structure on $X_{\sigma}(\mathbb{C})$.

In the sequel, we shall apply formula (4.2.1) to correspondences defined by incidence relations between some subvarieties of projective spaces. Correspondences of that kind play a key role in integral geometry and in Nevanlinna theory (see for instance [B-C], [St4]) as well as in analysis over homogeneous spaces (see for instance [Ge], in particular Vol. III, part 1, or [He]).

4.2.2. Joins. As a first application of formula (4.2.1), we shall compute the height of the join of two projective varieties.

Let $E_{1}$ and $E_{2}$ be two vector bundles on $S$, and $E=E_{1} \oplus E_{2}$ their direct sum. We define as follows a correspondence $C$ between $X=\mathbb{P}\left(E_{1}\right) \times_{S} \mathbb{P}\left(E_{2}\right)$ and $Y=\mathbb{P}(E)$. Let $p_{i}: X \rightarrow \mathbb{P}\left(E_{i}\right), i=1,2$, be the projection and $F$ the rank-two bundle $p_{1}^{*} \mathscr{O}_{E_{1}}(-1) \oplus p_{2}^{*} \mathscr{O}_{E_{2}}(-1)$ on $X$. Denote by $\pi: X \rightarrow S$ and $\pi_{i}: \mathbb{P}\left(E_{i}\right) \rightarrow S$ the projections; since $\mathscr{O}_{E_{i}}(-1)$ is a subbundle of $\pi_{i}^{*}\left(E_{i}\right), F$ is a subbundle of

$$
p_{1}^{*} \pi_{1}^{*} E_{1} \oplus p_{2}^{*} \pi_{2}^{*} E_{2}=\pi^{*} E .
$$

Then we let $f: C=\mathbb{P}_{X}(F) \rightarrow X$ be the projective bundle associated to $F$ and $g: C \rightarrow Y$ the map induced by the inclusion $F \rightarrow \pi^{*} E$. When $E_{1}$ and $E_{2}$ are trivial of rank $N_{1}+1$ and $N_{2}+1$ respectively, we may also describe $C$ as the reduced subscheme in $\mathbb{P}^{N_{1}} \times \mathbb{P}^{N_{2}} \times \mathbb{P}^{N_{1}+N_{2}+1}$ whose geometric points have 
homogeneous coordinates $\left(x_{0}: \ldots: x_{N_{1}}, y_{0}: \ldots: y_{N_{2}}, \lambda x_{0}: \ldots: \lambda x_{N_{1}}: \mu y_{0}:\right.$ $\left.\ldots: \mu y_{N_{2}}\right)$ with $(\lambda, \mu) \neq(0,0)$.

Given two cycles $Z_{1} \in Z_{p_{1}}\left(\mathbb{P}\left(E_{1}\right)\right)$ and $Z_{2} \in Z_{p_{2}}\left(\mathbb{P}\left(E_{2}\right)\right)$, we define their join to be the cycle

$$
Z_{1} \# Z_{2}=C_{*}\left(Z_{1} \times Z_{2}\right)
$$

in $Z_{p_{1}+p_{2}}(\mathbb{P}(E))$. When the cycles $Z_{1}$ and $Z_{2}$ are integral and one of them is flat over $S, Z_{1} \# Z_{2}$ is the cycle attached to the join scheme of $Z_{1}$ and $Z_{2}$ as defined in [A-K]. In particular, given $F_{1} \subset E_{1}$ and $F_{2} \subset E_{2}$ two nonzero subbundles, we have

$$
\mathbb{P}\left(F_{1}\right) \# \mathbb{P}\left(F_{2}\right)=\mathbb{P}\left(F_{1} \oplus F_{2}\right) .
$$

We suppose now that $E_{1}$ and $E_{2}$ are equipped with hermitian metrics and that $E_{1} \oplus E_{2}$ is equipped with the direct sum metric, and we denote by $h$ the projective heights on $\mathbb{P}\left(E_{1}\right), \mathbb{P}\left(E_{2}\right)$, and $\mathbb{P}\left(E_{1} \oplus E_{2}\right)$ associated to these hermitian vector bundles.

Proposition 4.2.2. For any two cycles $Z_{1}$ on $\mathbb{P}\left(E_{1}\right)$ and $Z_{2}$ on $\mathbb{P}\left(E_{2}\right)$ of dimensions $>0$, the following formulae hold:

$$
\operatorname{deg}_{K}\left(Z_{1} \# Z_{2}\right)=\operatorname{deg}_{K}\left(Z_{1}\right) \operatorname{deg}_{K}\left(Z_{2}\right)
$$

and

$$
h\left(Z_{1} \# Z_{2}\right)=h\left(Z_{1}\right) \operatorname{deg}_{K}\left(Z_{2}\right)+\operatorname{deg}_{K}\left(Z_{1}\right) h\left(Z_{2}\right) .
$$

Formula (4.2.4) for the degree of the join is well known (see for instance [Fu2], Examples 8.4.4, 8.4.5).

Proof. Let $\bar{Q}_{1}, \bar{Q}_{2}$, and $\bar{Q}$ be the canonical quotient hermitian bundles on $\mathbb{P}\left(E_{1}\right), \mathbb{P}\left(E_{2}\right)$, and $\mathbb{P}(E)$ respectively, and let $\widehat{c}(\bar{Q})$ be the total Chern class of $\bar{Q}$. From (4.2.1) we get

$$
\left(\widehat{c}(\bar{Q}) \mid Z_{1} \# Z_{2}\right)=\left(C^{*}(\widehat{c}(\bar{Q})) \mid Z_{1} \times Z_{2}\right) .
$$

We first show the equality

$$
C^{*}(c(Q))=c\left(Q_{1}\right) \times c\left(Q_{2}\right)
$$

in the rational algebraic Chow group $C H^{*}(X)_{\mathbb{Q}}$. We know from [G-S3], Proposition 3.1.4, (i), that the map

$$
C H^{*}(X)_{\mathbb{Q}} \rightarrow C H^{*}\left(X_{K}\right)_{\mathbb{Q}}
$$

is an isomorphism and therefore $C H^{*}(X)_{\mathbb{Q}}$ is generated by the classes $c_{i}\left(Q_{1}\right) \times$ $c_{j}\left(Q_{2}\right), 0 \leq i \leq \mathrm{rk} E_{1}, 0 \leq j \leq \mathrm{rk} E_{2}$. Let us write 


$$
C^{*}(c(Q))=\sum_{i, j} a_{i j} c_{i}\left(Q_{1}\right) \times c_{j}\left(Q_{2}\right), a_{i j} \in \mathbb{Q} \text {. }
$$

Using (4.2.6) (in $\left.\widehat{C H}^{0}(S)\right)$ and (2.3.18), we get

$$
\begin{aligned}
\operatorname{deg}_{K}\left(Z_{1} \# Z_{2}\right) & =\sum_{i, j} a_{i j} \operatorname{deg}_{K}\left(c_{i}\left(Q_{1}\right) \times c_{j}\left(Q_{2}\right) \mid Z_{1} \times Z_{2}\right) \\
& =\sum_{i, j} a_{i j} \operatorname{deg}_{K}\left(c_{i}\left(Q_{1}\right) \mid Z_{1}\right) \operatorname{deg}_{K}\left(c_{j}\left(Q_{2}\right) \mid Z_{2}\right)
\end{aligned}
$$

for all cycles $Z_{1}$ and $Z_{2}$. Applying this to $\mathbb{P}\left(F_{1}\right)$ and $\mathbb{P}\left(F_{2}\right)$, where $F_{1}$ and $F_{2}$ have codimension $i$ and $j$ respectively, we deduce from (4.2.3) that $a_{i j}=1$ for every $(i, j)$. This proves (4.2.7) and (4.2.4).

Now let

$$
x=C^{*}(\widehat{c}(\bar{Q}))-\widehat{c}\left(\bar{Q}_{1}\right) \times \widehat{c}\left(\bar{Q}_{2}\right)
$$

The image $z(x)$ of $x$ in $C H^{*}(X)_{\mathbb{Q}}$ vanishes by (4.2.7). For any imbedding $\sigma: K \hookrightarrow \mathbb{C}$, the form $\omega(x)$ is invariant under the action of the unitary group $U\left(\bar{E}_{1 \sigma}\right) \times U\left(\bar{E}_{2 \sigma}\right)$ upon $X_{\sigma}(\mathbb{C})$, therefore, by the discussion in 4.2.1, $\omega(x)$ is harmonic on $X(\mathbb{C})$ equipped with the Kähler form $p_{1}^{*} c_{1}\left(\overline{\mathscr{O}_{E_{1}}(1)}\right)+$ $p_{2}^{*} c_{1}\left(\overline{\mathscr{O}_{E_{2}}(1)}\right)$. From (4.2.7) we know that the cohomology class of $\omega(x)$ vanishes, therefore $\omega(x)=0$. By Lemma 2.1.1 we conclude that $x=a(\eta)$, where $\eta$ is a closed form. Using (4.2.6) (in $\widehat{C H}^{1}(S)$ ), we now get:

$$
h\left(Z_{1} \# Z_{2}\right)=h\left(Z_{1}\right) \operatorname{deg}_{K}\left(Z_{2}\right)+\operatorname{deg}_{K}\left(Z_{1}\right) h\left(Z_{2}\right)+\frac{1}{2} \int_{X(\mathbb{C})} \eta \delta_{Z_{1} \# Z_{2}} .
$$

On the other hand, when $Z_{1}=\mathbb{P}\left(F_{1}\right)$ and $Z_{2}=\mathbb{P}\left(F_{2}\right)$ for some nonzero subbundles $F_{1}$ and $F_{2}$ of $E_{1}$ and $E_{2}$, we have by (4.2.3) and Proposition 4.1.2, (ii):

$$
\begin{aligned}
h\left(Z_{1} \# Z_{2}\right) & =\widehat{\operatorname{deg}}\left(\bar{E}_{1} \oplus \bar{E}_{2}\right)-\widehat{\operatorname{deg}}\left(\bar{F}_{1} \oplus \bar{F}_{2}\right) \\
& =\left(\widehat{\operatorname{deg}}\left(\bar{E}_{1}\right)-\widehat{\operatorname{deg}}\left(\bar{F}_{1}\right)\right)+\left(\widehat{\operatorname{deg}}\left(\bar{E}_{2}\right)-\widehat{\operatorname{deg}}\left(\bar{F}_{2}\right)\right) \\
& =h\left(Z_{1}\right)+h\left(Z_{2}\right) .
\end{aligned}
$$

It follows that the integral $\int_{X(\mathbb{C})} \eta \delta_{Z_{1} \# Z_{2}}$ vanishes when $Z_{1_{K}}$ and $Z_{2_{K}}$ are linear subspaces of $\mathbb{P}\left(E_{1}\right)_{K}$ and $\mathbb{P}\left(E_{1}\right)_{K}$. Since $\eta$ is closed, this integral depends only on the cohomology class of $\delta_{Z_{1} \# Z_{2}}$, hence on the class of $Z_{1} \# Z_{2}$ in $C H^{*}\left(\mathbb{P}\left(E_{1} \oplus E_{2}\right)_{K}\right)$. As this group is generated by the classes of linear subspaces of the form $\mathbb{P}\left(F_{1} \oplus F_{2}\right)$, the integral always vanishes, and (4.2.5) follows.

4.2.3. A Bézout theorem for heights. Let $\bar{E}$ be an hermitian vector bundle of rank $N+1$ over $S$, and $X \in Z_{p}(\mathbb{P}(E))$ and $Y \in Z_{q}(\mathbb{P}(E))$ two effective cycles 
on the projective space of $E$. Assume that $p>0, q>0, p+q \geq N+1$, and that $X$ and $Y$ meet properly on $\mathbb{P}(E)_{K}$. Denote by $X$. $Y$ any cycle supported on $|X| \cap|Y|$ representing the product $[X][Y] \in C H_{|X| \cap|Y|}^{2 N+2-p-q}\left(\mathbb{P}^{N}\right)$ (see [Fu2] and 2.2). This cycle $X . Y$ is well defined up to the addition of $\sum_{\alpha} \operatorname{div}\left(f_{\alpha}\right)$, where each function $f_{\alpha}$ is defined on a variety contained in a closed fiber of $\mathbb{P}(E)$. It follows that the real number $h(X . Y)$ is independent of the choice of the representative $X . Y$ (by Proposition 3.2.1, (ii) and Proposition 4.1.2, (i)).

Theorem 4.2.3. Assume that $\bar{E}$ is the trivial hermitian vector bundle $\overline{\mathscr{O}}_{S}^{\oplus(N+1)}$. Then the following inequality holds:

$$
\begin{aligned}
h(X . Y) \leq & h(X) \operatorname{deg}_{K}(Y)+\operatorname{deg}_{K}(X) h(Y) \\
& +[K: \mathbb{Q}] a(N, p, q) \operatorname{deg}_{K}(X) \operatorname{deg}_{K}(Y),
\end{aligned}
$$

where

$$
a(N, p, q)=\sigma_{p+q-1}-\sigma_{p+q-N-2}+\frac{2 N+2-p-q}{2} \log 2 .
$$

As mentioned in the introduction, special cases of Theorem 4.2.3 where $X$ or $Y$ is an hypersurface, or more generally a complete intersection, have already been proved by Nesterenko ([N1], Lemma 4), Philippon ([P1], Proposition 2.6; [P3], Théorème 2), and Faltings ([Fa2], Proposition 2.17). Nesterenko and Philippon use heights of cycles defined by means of Chow forms; but these turn out to differ from the heights $h_{F}$ and $h$ by a multiple of the degree, see [So2], [P2], and 4.3, infra.

Proof. Let $D \subset \mathbb{P}(E \oplus E)$ be the image of the "diagonal" embedding $j: \mathbb{P}(E) \rightarrow$ $\mathbb{P}(E \oplus E)$ defined by the bundle morphism $i d_{E} \oplus i d_{E}: E \rightarrow E \oplus E$, and $X \# Y$ the join of $X$ and $Y$. Since $X$ and $Y$ meet properly on $\mathbb{P}(E)_{K}$, the cycles $X \# Y$ and $D$ meet properly on $\mathbb{P}(E \oplus E)_{K}$. Furthermore

$$
j_{*}(X . Y)=(X \# Y) . D .
$$

By this we mean that for any choice of the representative $X . Y$ of $[X][Y] \in$ $C H_{*}(|X| \cap|Y|), j_{*}(X . Y)$ is a representative of $[X \# Y][D] \in C H_{*}(|X \# Y| \cap|D|)$. This fact follows from [Fu2], Example 8.4.5. (Fulton's argument is formulated for joins in projective spaces over a field, but extends immediately to the present situation.)

Let $\bar{L}=\overline{\mathscr{O}_{E \oplus E}(1)}$ be the canonical hermitian line bundle on $\mathbb{P}(E \oplus E)$. By Proposition 3.2.1, (iii) and (4.2.10) we get

$$
h_{j^{*}(\bar{L})}(X . Y)=h_{\bar{L}}((X \# Y) \cdot D) .
$$

The isomorphism $j^{*} \mathscr{O}_{E \oplus E}(-1) \stackrel{\sim}{\rightarrow} \mathscr{O}_{E}(-1)$ divides scalar products by 2 . Indeed it maps the triple $(x, v, v), x \in \mathbb{P}\left(E_{\mathbb{C}}\right), v \in E_{\mathbb{C}}$, to $(x, v)$, and $\|(v, v)\|^{2}=$ $2\|v\|^{2}$. By (3.2.4) it follows that 


$$
h_{j^{*}(\bar{L})}(X . Y)=h_{F}(X . Y)+[K: \mathbb{Q}] \frac{p+q-N-1}{2}(\log 2) \operatorname{deg}_{K}(X . Y)
$$

if $p+q>N+1$ and

$$
h_{j^{*}(\bar{L})}(X . Y)=h_{F}(X . Y)
$$

if $p+q=N+1$. All this was valid for an arbitrary $\bar{E}$. Assume now that $\bar{E}=\overline{\mathscr{\sigma}}_{S}^{N+1}$. Then $\mathbb{P}(E \oplus E)$ gets identified with $\mathbb{P}_{S}^{2 N+1}$. Let $\left(X_{0}, \ldots, X_{N}\right.$, $\left.Y_{0}, \ldots, Y_{N}\right)$ be the canonical homogeneous coordinates on $\mathbb{P}_{S}^{2 N+1}$, and let $H_{i} \subset \mathbb{P}_{S}^{2 N+1}$ be the hyperplane of equation $X_{i}=Y_{i}$. Clearly $D$ is the intersection of $H_{0}, \ldots, H_{N}$. When $Z$ is any effective cycle on $\mathbb{P}_{S}^{2 N+1}$ flat over $S$ and meeting $H_{i}$ properly, we get from Proposition 3.2.1, (iv) that

$$
h_{\bar{L}}\left(Z . H_{i}\right) \leq h_{\bar{L}}(Z)+[K: \mathbb{Q}] \frac{\log 2}{2} \operatorname{deg}_{K}(Z)
$$

since the section $s$ of $L$ corresponding to $X_{i}-Y_{i}$ has norm square at the point $\left(x_{0}: \ldots: x_{N}: y_{0}: \ldots: y_{N}\right) \in \mathbb{P}_{S \sigma}^{2 N+1}(\mathbb{C}) \simeq \mathbb{P}^{2 N+1}(\mathbb{C})$

$$
\frac{\left|x_{i}-y_{i}\right|^{2}}{\left|x_{0}\right|^{2}+\cdots+\left|x_{N}\right|^{2}+\left|y_{0}\right|^{2}+\cdots+\left|y_{N}\right|^{2}} \leq 2 .
$$

On the other hand, for any cycle $Z$ supported in a closed fiber of $\mathbb{P}^{2 N+1} \rightarrow S$, we have

$$
h_{\bar{L}}\left(Z . H_{i}\right)=h_{\bar{L}}(Z)
$$

according to (3.1.3), since $\left[H_{i}\right]=c_{1}(L)$. Therefore (4.2.13) holds for any cycle $Z$ on $\mathbb{P}^{2 N+1}$ such that $Z_{K}$ is effective and meets $H_{i}$ properly (on $\mathbb{P}_{K}^{2 N+1}$ ).

Applying (4.2.13) $N+1$ times, it follows that

$$
h_{\bar{L}}((X \# Y) . D) \leq h_{\bar{L}}(X \# Y)+[K: \mathbb{Q}] \frac{N+1}{2} \log (2) \operatorname{deg}_{K}(X \# Y) .
$$

Indeed, if $p+q>N+1$ (resp., $p+q=N+1$ ), $|X \# Y|_{K} \cap|D|_{K}$ has dimension $p+q-N-2$ (resp., is empty), so that $|X \# Y|_{K} \cap H_{0_{K}} \cap \cdots \cap H_{i_{K}}$ has dimension $p+q-i-2$ for any $i \in\{0, \ldots, N\}$ (resp., has dimension $N-i-1$ for any $i \in\{1, \ldots, N\})$.

Combining (4.2.11), (4.2.12), (4.2.14), Proposition 4.2.2 (applied to $E_{1}=$ $E_{2}=E, Z_{1}=X$, and $Z_{2}=Y$ ), and using Proposition 4.1.2, (i) to compare Faltings height with the projective height, Theorem 4.2 .3 follows.

The use of the join to reduce intersection of cycles in projective space to the intersection of one cycle with a linear subspace goes back to Gaeta ([Ga]) and has been used since by several authors, in relation with Bézout's theorem ([Bo-V], [V], [Fu2], Examples 8.4.4 - 8.4.6, [Fu3]). 
Corollary 4.2.4. Let $h$ be the projective height on cycles in $\mathbb{P}_{K}^{N}\left(\simeq \mathbb{P}(E)_{K}\right)$ defined by the trivial hermitian vector bundle $\bar{E}$. For any two effective cycles $X \in Z_{p}\left(\mathbb{P}_{K}^{N}\right)$ and $Y \in Z_{q}\left(\mathbb{P}_{K}^{N}\right)$ which meet properly $(p+q \geq N)$, the following inequality on the height of the intersection cycle X.Y holds:

$$
\begin{aligned}
h(X . Y) \leq & h(X) \operatorname{deg}_{K}(Y)+\operatorname{deg}_{K}(X) h(Y) \\
& +[K: \mathbb{Q}]\left(\sigma_{p}+\sigma_{q}-\sigma_{p+q-N}+\frac{2 N-p-q}{2} \log 2\right) \\
& \times \operatorname{deg}_{K}(X) \cdot \operatorname{deg}_{K}(Y) .
\end{aligned}
$$

Proof. According to [Fu2], Corollary 12.2 (a) (see also 20.1 and 20.2), there exists an effective cycle $\bar{X} \cdot \bar{Y}$ supported by $|\bar{X}| \cap|\bar{Y}|$ representing $[X][Y]$. Then $\bar{X} . \bar{Y}-\overline{X . Y}$ is an effective cycle supported by the closed fibers of $\mathbb{P}_{S}^{N} \rightarrow S$; therefore

$$
h(X . Y)=h(\overline{X . Y}) \leq h(\bar{X} . \bar{Y}) .
$$

Combined with (4.2.9) applied to $\bar{X}$ and $\bar{Y}$, this gives (4.2.15).

Remarks. When comparing the classical Bézout theorem with the inequality above, we may say that if the degree represents a zeroth order information about a cycle, then the height is first order information; see 5.4.1 below for a more precise statement.

It is also an illustration of the point of view of Northcott [No] that the height measures the "complexity" of the cycle.

\subsection{The height of Chow forms and resultants.}

4.3.1. Definitions. Let $E$ be a vector bundle of rank $N+1$ over $S, r \in[0, N]$ an integer, and $\mathbf{d}=\left(d_{0}, \ldots, d_{r}\right)$ an $(r+1)$-tuple of positive integers. Let $\pi: \mathbb{P}=\mathbb{P}(E) \rightarrow S$ be the projective bundle of $E$, and, for any $i$, let $S^{d i} \stackrel{\vee}{E}$ be the $d_{i}$-th symmetric product of $\stackrel{\vee}{E}$. It is defined as the quotient of $\quad \stackrel{\vee}{E^{\otimes d_{i}}}$, defined by the coinvariant under the action of the symmetric group. We let

$$
\mathbb{P}_{i}=\mathbb{P}\left(\left(S^{d_{i}} \stackrel{\vee}{E}\right)^{\vee}\right) \text { and } \stackrel{\vee}{\mathbb{P}_{i}}=\mathbb{P}\left(S^{d_{i}} \stackrel{\vee}{E}\right)
$$

The scheme $\stackrel{\vee}{\mathbb{P}}_{i}$ "parametrizes" the horizontal divisors of relative degree $d_{i}$ on $\mathbb{P}$, as well as the hyperplanes in $\mathbb{P}_{i}$. The schemes $\mathbb{P}_{i}$ and $\stackrel{\vee}{\mathbb{P}}$ have relative dimensions

$$
N_{i}=\operatorname{rk}\left(S^{d_{i}} \stackrel{\vee}{E}\right)-1=\left(\begin{array}{c}
N+d_{i} \\
d_{i}
\end{array}\right)-1 .
$$

The product $\mathbb{P}_{i} \times{ }_{S} \mathbb{P}_{i}^{\vee}$ contains the incidence subscheme $I_{i}$, that is, the subscheme, smooth over $S$, whose points in any field $k$ over $S$ are pairs 
$(x, u)$, where $x \in \mathbb{P}_{i}(k), u \in \mathbb{P}_{i}^{\vee}(k)$, and $\tilde{u}(\tilde{x})=0$ for any representative $\tilde{x} \in\left(S^{d_{i}} \stackrel{\vee}{E}\right)^{\vee}{\underset{\mathscr{O}}{K}}_{\otimes} k$ and $\tilde{u} \in S^{d_{i}} \stackrel{\vee}{E} \stackrel{\mathscr{O}}{K}_{\otimes} k$ of $x$ and $u$ respectively. Let $\Gamma=$ $I_{0} \times{ }_{S} \cdots \times_{S} I_{r}$ be the product of these schemes. The projection maps

$$
f: \Gamma \rightarrow \prod_{i=0}^{r} \mathbb{P}_{i}:=\mathbb{P}_{0} \times_{S} \cdots \times_{S} \mathbb{P}_{r}
$$

and

$$
g: \Gamma \rightarrow \prod_{i=0}^{r} \mathbb{P}_{i}^{\vee}:=\mathbb{P}_{0}^{\vee} \times_{S} \cdots \times_{S} \mathbb{P}_{r}^{\vee}
$$

are smooth and proper. Finally, let $v_{i}: \mathbb{P} \rightarrow \mathbb{P}_{i}$ be the Veronese embedding, attached to the very ample line bundle $\mathscr{O}\left(d_{i}\right)$ on $\mathbb{P}$. If $v: \mathbb{P} \rightarrow \prod_{i=0}^{r} \mathbb{P}_{i}$ is the product of the $v_{i}$ 's, we get a diagram:

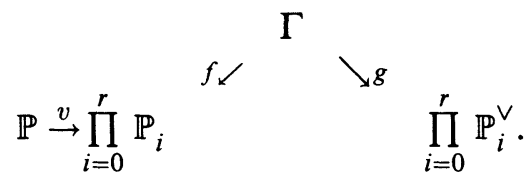

Given any cycle $Z \in Z_{r+1}(\mathbb{P})$, we define its (generalized) Chow divisor to be

$$
\mathrm{Ch}_{\mathbf{d}}(Z)=g_{*} f^{*} v_{*}(Z)=\Gamma_{*} v_{*}(Z)
$$

This cycle is effective when $Z$ is effective and has codimension one on $\prod_{i=0}^{r} \mathbb{P}_{i}^{\vee}$.

Lemma 4.3.1. When $Z$ is flat over $S$, resp. flat over $S$ and irreducible, the same is true for $\mathrm{Ch}_{d_{0}, \ldots, d_{r}}(Z)$.

Proof. Assume first that $Z$ is an irreducible cycle on $\mathbb{P}$, i.e., $Z$ is an integral subscheme of $\mathbb{P}$. Observe that $Z$ is flat over $S$ iff its generic point lies over the generic point $\operatorname{Spec}(K) \in S$. Since $f$ is smooth with geometrically connected fibers, the cycle $f^{*} v_{*}(Z)$ is also irreducible, and its generic point lies above that of $v_{*}(Z)$, hence also above $\operatorname{Spec}(K)$. Write $g_{*} f^{*} v_{*}(Z)=n W$, where $W$ is the integral subscheme of $\prod_{i=0}^{r} \mathbb{P}_{i}^{\vee}$ which has the same support as the scheme theoretic image of the irreducible scheme $f^{*} v_{*}(Z)$ under $g$. Since the generic point of $f^{*} v_{*}(Z)$ lies over $\operatorname{Spec}(K)$ the same is true for $W$; i.e., $W$ is flat over $S$. Note that the integer $n$ will be zero if $W$ has codimension greater than 1 ; otherwise it equals the degree of the extension $K\left(f^{*} v_{*}(Z)\right) \mid K(W)$. Since proper pushforward and flat pullback of cycles commute ([Fu2], Proposition 1.7, and $\S 20.1$, we may compute $n$ after base changing by the map $\operatorname{Spec}(K) \rightarrow S$. Then the assertion that $C h_{\mathrm{d}}(Z)$ is irreducible, i.e., that $n=1$, is essentially a classical result about Chow forms over fields of characteristic zero, which may be proved as follows. 
Let $v^{\prime}=\mathbb{P} \rightarrow \prod_{i \geq 1} \mathbb{P}_{i}, f^{\prime}: \prod_{i \geq 1} I_{i} \rightarrow \prod_{i \geq 1} \mathbb{P}_{i}$, and $g^{\prime}: \prod_{i \geq 1} I_{i} \rightarrow \prod_{i \geq 1} \mathbb{P}_{i}^{\vee}$ be the maps defined analogously to $v, f$, and $g$. Then $f^{\prime-1} v^{\prime}(Z)$ is an integral scheme, and we have a commutative diagram:

$$
\begin{gathered}
f^{-1} v(Z) \stackrel{h}{\longrightarrow} \prod_{i \geq 0} \mathbb{P}_{i}^{\vee} \\
\downarrow \pi \quad \downarrow_{p} \\
f^{\prime-1} v^{\prime}(Z) \stackrel{h^{\prime}}{\longrightarrow} \prod_{i \geq 1} \mathbb{P}_{i}^{\vee}
\end{gathered}
$$

in which $h$ and $h^{\prime}$ are the maps induced by $g$ and $g^{\prime}, p$ is the obvious projection, and $\pi$ is induced by the corresponding projection $\prod_{i \geq 0} I_{i} \rightarrow \prod_{i \geq 1} I_{i}$. We want to show that $h$ is generically injective. Suppose that $\xi: \operatorname{Spec}(F) \rightarrow$ $\prod_{i \geq 1} \mathbb{P}_{i}^{\vee}$ is a geometric generic point, and let $D_{i} \subset \mathbb{P}_{F}$ be the corresponding degree $d_{i}$ hypersurface for $i=1, \ldots, r$. Since $Z_{K} \subset \mathbb{P}_{K}$ is nonempty and has dimension $r, Z_{F} \cap D_{1} \cap \cdots \cap D_{r} \subset \mathbb{P}_{F}$ is nonempty. But this is canonically isomorphic (via the morphisms $v^{\prime}$ and $f^{\prime}$ ) to $h^{\prime-1}(\xi)$. Hence $h^{\prime}$ is a dominant morphism between varieties of the same dimension and is therefore generically finite, of degree $\lambda=\operatorname{deg}(Z) d_{1} \cdots d_{r}$. Furthermore, since $h^{\prime}$ is a morphism of varieties of characteristic zero it is generically étale. Thus

$$
h^{\prime-1}(\xi)=\left\{P_{1}, \ldots, P_{\lambda}\right\} \subset v^{\prime}(\mathbb{P}) \times \xi=v^{\prime}\left(\mathbb{P}_{F}\right)
$$

with the $P_{i}$ distinct. If we pull back diagram (4.3.4) along $\xi=\operatorname{Spec}(F) \rightarrow$ $\prod_{i \geq 1} \mathbb{P}_{i}^{\vee}$ :

$$
\begin{aligned}
f^{-1} v(Z)_{\xi} & \longrightarrow \mathbb{P}_{0, F}^{\vee} \\
\downarrow \pi_{\xi} & \downarrow \\
\left\{P_{1}, \ldots, P_{\lambda}\right\} & \longrightarrow \operatorname{Spec}(F)
\end{aligned}
$$

we find that $f^{-1} v(Z)_{\xi} \subset \mathbb{P}_{0, F} \times \mathbb{P}_{0, F}^{\vee}$ is $\bigcup_{i=1}^{\lambda} P_{i} \times H_{i}$, where $H_{i}$ is the hyperplane dual to $P_{i}$. Since the $P_{i}$ are distinct, so are the $H_{i}$, and thus $f^{-1} v(Z)_{\xi}$ maps injectively to $\mathbb{P}_{0, F}^{\vee}$.

When $Z$ is flat over $S$ but not necessarily irreducible, the above argument applies to each of its components.

4.3.2. Remarks and examples. (i) The definition of the generalized Chow divisor may be extended by replacing the base scheme $S$ by any Noetherian regular scheme $T$. Namely, for any vector bundle $E$ of rank $N+1$ over $T$, we can 
still define $\mathbb{P}_{i}$ and $\stackrel{\vee}{\mathbb{P}}$ by (4.3.1) and consider the diagram (4.3.2) (where all the products are over $T$ ); then for any effective cycle $Z$ on $\mathbb{P}=\mathbb{P}(E)$ with relative dimension $\operatorname{dim}_{S} Z=\operatorname{dim} Z-\operatorname{dim} S$ equal to $r$, formula (4.3.3) defines a generalized Chow divisor $\mathrm{Ch}_{\mathrm{d}}(Z)$ on $\prod_{i=0}^{r} \stackrel{\mathrm{P}}{\mathbb{P}}_{i}$.

The compatibility of flat pull-back and proper push-forward imply that the formation of the Chow divisor commutes with flat base change. Namely, if $T^{\prime}$ is another Noetherian scheme, for any flat morphism $T^{\prime} \rightarrow T$, we have the following equality of divisors in $\left(\prod_{i=0}^{r} \stackrel{\vee}{\mathbb{P}_{i}}\right)_{T^{\prime}}$ :

$$
\mathrm{Ch}_{\mathbf{d}}\left(Z_{T^{\prime}}\right)=\mathrm{Ch}_{\mathbf{d}}(Z)_{T^{\prime}}
$$

It follows from the constructions of Mumford in [M-F], 5.3-5.4 (see also [K$M]$ and [Fo]), that if $Z$ is any irreducible cycle in $\mathbb{P}$ defined by a subscheme flat and of relative dimension $r$ over $T$, the Chow divisor $\mathrm{Ch}_{\mathrm{d}}(Z)$ is flat over $T$ and formula (4.3.5) holds for a not necessarily flat base change $T^{\prime} \rightarrow T$. (In fact, using Mumford's construction, one may associate a flat Chow divisor $\mathrm{Ch}_{\mathrm{d}}(Z)$ to any subscheme $Z$ of $\mathbb{P}$ flat over $T$, without any regularity assumption on the base $T$, in a way compatible with any base change. Moreover, this definition is compatible with our intersection theoretic definition, as follows from [M-F], Lemma 5.9, which immediately extends to schemes.)

Formula (4.3.5) also holds when $T^{\prime}$ and $T$ are regular noetherian schemes and the morphism $i: T^{\prime} \rightarrow T$ is a closed regular embedding such that

$$
\operatorname{dim}_{T^{\prime}}|Z|_{T^{\prime}}=\operatorname{dim}_{T}|Z|,
$$

if now $Z_{T^{\prime}}$ and $\mathrm{Ch}_{\mathrm{d}}(Z)_{T^{\prime}}$ denote the restrictions of $Z$ and $\mathrm{Ch}_{\mathrm{d}}(Z)$, i.e., their image under the Gysin homomorphisms defined by $i$. This follows from the compatibility of Gysin homomorphisms with flat pull-back and proper pushforward ([Fu2], $\S \S 6.2$ and 20.3).

(ii) When $T=\operatorname{Spec}(k)$, for some field $k$ of characteristic zero, and $d_{0}=$ $\cdots=d_{r}=1$, the Chow divisor $\mathrm{Ch}_{\mathrm{d}}(Z)$ is the divisor of the classical Chow form of $Z$. Indeed, it is irreducible if $Z$ is so (by the same argument as in Lemma 4.3.1), and its geometric points parametrize $(r+1)$-tuples of hyperplanes in $\mathbb{P}$ whose intersection meets the support of $Z$ (see [C-Wa], pp. 693-694 and [Sa], I.9.4; it follows from this last reference that $\mathrm{Ch}_{(1, \ldots, 1)}(Z)$ is the classical Chow divisor of $Z$ when $k$ is algebraically closed of any characteristic).

The introduction of generalized Chow divisors, for arbitrary $d_{i}$ 's, is due to Philippon [P1]. When $T=\operatorname{Spec}(k)$ and $k$ has characteristic zero, $\operatorname{Ch}_{\mathbf{d}}(Z)$ is still irreducible when $Z$ is so, and has a geometric interpretation which generalizes the one for $\mathrm{Ch}(Z)$ above: the geometric points of its support parametrize $(r+1)$-tuples of hypersurfaces of degree $d_{0}, \ldots, d_{r}$ in $\mathbb{P}$ whose intersection meets the support of $Z$ (see the proof of Lemma 4.3.1).

(iii) Suppose that $T=\operatorname{Spec}(A)$, where $A$ is a field or a principal ideal domain. Then $E$ may be trivialized, and we get identifications: 


$$
\mathbb{P} \simeq \mathbb{P}_{A}^{N} \text { and } \prod_{i=0}^{r} \stackrel{\vee}{\mathbb{P}_{i}} \simeq \prod_{i=0}^{r} \mathbb{P}_{A}^{\left(\begin{array}{c}
N+d_{i} \\
d_{i}
\end{array}\right)-1}
$$

Therefore, $\mathrm{Ch}_{\mathbf{d}}(Z)$ is the divisor of a multihomogeneous form $\Phi_{d, Z}$ with coefficients in $A$ in $\sum_{i=0}^{r}\left(\begin{array}{c}N+d_{i} \\ d_{i}\end{array}\right)$ variables (the coefficients of the "generic" homogeneous polynomials of degree $d_{0}, \ldots, d_{r}$ in $N+1$ variables), well defined up to the multiplication by a unit in $A$. This form generalizes the classical Chow form and was considered by Nesterenko [N1] and Philippon [P1] (when $Z$ is irreducible, it coincides with the "forme éliminante" of [P1], p. 23).

(iv) According to Lemma 4.3.1 (or to (i) above), when $Z$ is an effective cycle on $\mathbb{P}$ flat over $S, \mathrm{Ch}_{\mathrm{d}}(Z)$ is the flat divisor extending the divisor $\mathrm{Ch}_{\mathrm{d}}\left(Z_{K}\right)$ in $\left(\prod_{i=0}^{r} \mathbb{P}_{i}\right)_{K}$. On the other hand, when $Z$ is supported in a closed fiber $\pi^{-1}(x)=\mathbb{P}_{x}$ of $\mathbb{P}$ over $S$, where it has degree $\delta$, one finds:

$$
\mathrm{Ch}_{\mathrm{d}}(Z)=\delta d_{0} \cdots d_{r}\left(\prod_{i=0}^{r} \stackrel{\vee}{\mathbb{P}_{i}}\right)_{x} .
$$

This is easy to prove directly, but also follows from the identity (4.3.6) below.

(v) Consider the case where $K=\mathbb{Q}, E$ is trivial, and $r=N$. Then $\mathbb{P}=\mathbb{P}_{\mathbb{Z}}^{N}$, and $\mathrm{Ch}_{\mathbf{d}}\left(\mathbb{P}_{\mathbb{Z}}^{N}\right)$ is the divisor of a multihomogeneous polynomial with integer coefficients $R_{\mathrm{d}}$, whose variables are the coefficients of the "generic" homogeneous polynomials of degrees $d_{0}, \ldots, d_{N}$ in $N+1$ variables. This polynomial, well defined up to a sign, coincides with the classical resultant of $N+1$ homogeneous polynomials of degrees $d_{0}, \ldots, d_{N}$ in $N+1$ variables ([Me]; [Wa], Chapter XI). Indeed it is irreducible over $\mathbb{Z}$ (by Lemma 4.3.1), and its complex zeros parametrize $(N+1)$-tuples of complex homogeneous polynomials of this type which have a common zero in $\mathbb{P}^{N}(\mathbb{C})$. It immediately follows from (ii) together with the compatibility of $\mathrm{Ch}_{\mathrm{d}}$ with base change described in (i) that, as is classically known, $R_{\mathrm{d}}$ is absolutely irreducible and that its set of zeros in any algebraically closed field $k$ parametrizes $(N+1)$-tuples of polynomials in $k\left[X_{0}, \ldots, X_{N}\right]_{d_{0}}, \ldots, k\left[X_{0}, \ldots, X_{N}\right]_{d_{N}}$ which have a common zero in $\mathbb{P}^{N}(k)$.

\subsubsection{The height of generalized Chow divisors.}

4.3.3.1. We keep the notation of 4.3 .1 and assume that $E$ is equipped with an hermitian metric $h$. We endow $\stackrel{\vee}{E}$ with the dual metric $\stackrel{\vee}{h}$, and for any integer $d$ we endow $S^{d} \stackrel{\vee}{E}$ with the quotient metric of the metric $\stackrel{\vee}{h}$ od on $\stackrel{\vee}{E}{ }^{\otimes d}$, and $\left(S^{d} \stackrel{\vee}{E}\right)^{\vee}$ with the dual metric. We denote by $\bar{Q}_{i}$ the pull-back to $\prod_{i=0}^{r} \mathbb{P}_{i}^{\vee}$ of the canonical hermitian quotient bundle on $\mathbb{P}_{i}^{\vee}=\mathbb{P}\left(S^{d_{i}} \stackrel{\vee}{E}\right)$ and by $\widehat{c}\left(\bar{Q}_{i}\right)$ its 
total Chern class. Given any divisor $D$ on $\prod_{i=0}^{r} \mathbb{P}_{i}^{\vee}$, we define its height to be

$$
h(D)=\widehat{\operatorname{deg}}\left(\prod_{i=0}^{r} \widehat{c}\left(\bar{Q}_{i}\right) \mid D\right) \in \mathbb{R}
$$

it is a normalized variant of the multiheight considered in §3.1 (cf. Lemma 4.3.4 below). We shall also consider the multidegrees of $D$, defined as:

$\operatorname{deg}_{i}(D)=\operatorname{deg}_{K}\left(c_{N_{0}}\left(Q_{0}\right) \cdots c_{N_{i-1}}\left(Q_{i-1}\right) c_{N_{i}-1}\left(Q_{i}\right) c_{N_{i+1}}\left(Q_{i+1}\right) \cdots c_{N_{r}}\left(Q_{r}\right) \mid D\right) \in \mathbb{Z}$

Let $\overline{\mathscr{O}(1)}$ be the canonical hermitian line bundle of degree one on $\mathbb{P}=\mathbb{P}(E)$.

Theorem 4.3.2. Let $Z \in Z_{r+1}(\mathbb{P})$. The Faltings height of $Z$ (resp. the degree of $Z$ ) and the height (resp. the multidegrees) of its generalized Chow form $\mathrm{Ch}_{\mathbf{d}}(Z)$ satisfy the following proportionality relations:

$$
h\left(\mathrm{Ch}_{\mathrm{d}}(Z)\right)=\left(\prod_{j=0}^{r} d_{j}\right) h_{\overline{\mathscr{Q}(1)}}(Z)
$$

and

$$
\operatorname{deg}_{i}\left(\operatorname{Ch}_{\mathbf{d}}(Z)\right)=\left(\prod_{\substack{j=0 \\ j \neq i}}^{r} d_{j}\right) \operatorname{deg}_{K}(Z), \quad 0 \leq i \leq r .
$$

The last identity is classical when $d_{0}=\cdots=d_{r}=1$ (see [C-Wa], pp. 693694). It is due in general to Philippon ([P1], p. 15, Remarques, 1)). The identity (4.3.6) is due to Soule [So2] and Philippon [P2] when $\bar{E}$ is trivial and $d_{0}=\cdots=d_{r}=1$.

The proof of Theorem 4.3 .2 will be based on Lemma 4.2.1 on correspondences, combined with the following:

Proposition 4.3.3. Let $\bar{F}$ be an hermitian vector bundle on $S, \overline{\mathscr{O}(1)}_{F}$ the canonical hermitian line bundle on $\mathbb{P}(F), \bar{Q}_{F^{\vee}}$ the canonical hermitian quotient bundle on $\mathbb{P}\left(F^{\vee}\right)$, and I the incidence correspondence between $\mathbb{P}(F)$ and $\mathbb{P}\left(F^{\vee}\right)$. Then the following equality holds in $\widehat{C H}^{*}(\mathbb{P}(F))_{\mathbb{Q}}$ :

$$
I^{*}\left(\widehat{c}\left(\bar{Q}_{F}\right)\right)=\widehat{c}\left(\overline{\mathscr{O}_{F}(1)}\right) .
$$

Proof of Proposition 4.3.3. Consider the commutative diagram

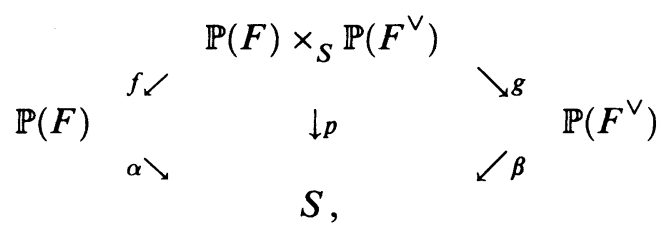

the canonical exact sequences 


$$
0 \rightarrow \mathscr{O}_{F}(-1) \rightarrow \alpha^{*} F \rightarrow Q_{F} \rightarrow 0
$$

and

$$
0 \rightarrow \mathscr{O}_{F^{\vee}}(-1) \rightarrow \beta^{*} F^{\vee} \rightarrow Q_{F^{\vee}} \rightarrow 0
$$

on $\mathbb{P}(F)$ and $\mathbb{P}\left(F^{\vee}\right)$ respectively, and the dual exact sequence on $\mathbb{P}(F)$

$$
0 \rightarrow Q_{F}^{\vee} \rightarrow \alpha^{*} F^{\vee} \rightarrow \mathscr{O}_{F}(1) \rightarrow 0 .
$$

The divisor $I$ in $\mathbb{P}(F) \times_{S} \mathbb{P}\left(F^{\vee}\right)$ is defined by the vanishing of the composite map

$$
g^{*}\left(\mathscr{O}_{F^{\vee}}(-1)\right) \rightarrow p^{*}\left(F^{\vee}\right) \rightarrow f^{*}\left(\mathscr{O}_{F}(1)\right) .
$$

The homology of the complex of length two obtained by restricting the sequence (4.3.7) to $I$ is

$$
\begin{aligned}
H & =\operatorname{coker}\left(g^{*}\left(\mathscr{O}_{F^{\vee}}(-1)\right) \rightarrow f^{*}\left(Q_{F}^{\vee}\right)\right) \\
& =\operatorname{ker}\left(g^{*}\left(Q_{F^{\vee}}\right) \rightarrow f^{*}\left(\mathscr{O}_{F}(1)\right)\right) .
\end{aligned}
$$

It follows that we may identify $f: I \rightarrow \mathbb{P}(F)$ with the projective bundle $\mathbb{P}\left(f^{*}\left(Q_{F}^{\vee}\right)\right) \rightarrow \mathbb{P}(F)$ and $H$ with the quotient bundle on this projective bundle. Furthermore, we have an exact sequence on $I$ :

$$
\mathscr{E}: 0 \rightarrow H \rightarrow g^{*}\left(Q_{F^{\vee}}\right) \rightarrow f^{*}\left(\mathscr{O}_{F}(1)\right) \rightarrow 0 .
$$

We equip all bundles with the metric induced by $\bar{F}$ and we let $\widetilde{c}(\mathscr{E})$ be the total Bott-Chern class of $\mathscr{E}$ for these choices of metrics. We get from (2.1.4) and the projection formula

$$
\begin{aligned}
I^{*}\left(\widehat{c}\left(\bar{Q}_{F^{\vee}}\right)\right) & =f_{\mid I *} g^{*} \widehat{c}\left(\bar{Q}_{F^{\vee}}\right) \\
& =f_{\mid I *}\left(\widehat{c}(\bar{H}) \widehat{c}\left(f^{*} \overline{\mathscr{O}_{F}(1)}\right)\right)-a\left(f_{\mid I *} \widetilde{c}(\mathscr{C})\right) \\
& =f_{\mid I *}(\widehat{c}(\bar{H})) \hat{c}\left(\overline{\mathscr{O}_{F}(1)}\right)-a\left(f_{\mid I *} \widetilde{c}(\mathscr{E})\right) .
\end{aligned}
$$

Since the rank of $H$ and the relative dimension of $f_{\mid I}$ have the same value $\operatorname{rank}(F)-2$, the class $f_{\mid I *}(\widehat{c}(\bar{H}))$ lies in $\widehat{C H}^{0}(\mathbb{P}(F))=\mathbb{Z}$. It is equal to one as can be checked on the generic fiber. Furthermore $\widetilde{c}(\mathscr{E})$ is zero in degrees bigger than $\operatorname{rank}(F)-2$, therefore $f_{*}(\widetilde{c}(\mathscr{C}))$ is a smooth function on $\mathbb{P}(F)(\mathbb{C})$. For any embedding $\sigma: K \rightarrow \mathbb{C}$, its restriction to $\mathbb{P}(F)_{\sigma}(\mathbb{C})$ is invariant under the action of the unitary group of $\bar{F}_{\sigma}$, and therefore must be a constant $\lambda_{\sigma} \in \mathbb{R}$. Indeed, the short exact sequence of hermitian holomorphic vector bundles on $I_{\sigma}(\mathbb{C})$ defined by $\mathscr{E}$ is $U\left(\overline{F_{\sigma}}\right)$-equivariant, as well as the map $f_{\sigma}: I_{\sigma}(\mathbb{C}) \rightarrow \mathbb{P}(F)_{\sigma}(\mathbb{C})$. The function $\sigma \mapsto \lambda_{\sigma}$ defines an element $\lambda \in A^{00}\left(S_{\mathbb{R}}\right)$, and we have

$$
I^{*}\left(\widehat{c}\left(\bar{Q}_{F^{\vee}}\right)\right)=\hat{c}\left(\overline{\mathscr{O}_{F}(1)}\right)-\alpha^{*} a(\lambda)
$$


To prove that $a(\lambda)=0$ in $\widehat{C H}^{1}(S)_{\mathbb{Q}}$, choose a line bundle $L$ contained in $F$ and let $Z=\mathbb{P}(L) \in Z_{1}(\mathbb{P}(F))$ be the corresponding cycle in $\mathbb{P}(F)$. From Lemma 4.2.1 we get

$$
\left(\widehat{c}\left(\bar{Q}_{F^{\vee}}\right) \mid I_{*}(Z)\right)=\left(\widehat{c}\left(\overline{\mathscr{O}(1)}_{F}\right)-\alpha^{*} a(\lambda) \mid Z\right)=\widehat{c}\left(\bar{L}^{\vee}\right)-a(\lambda) .
$$

On the other hand, since $I_{*}(Z)$ is the irreducible cycle $\mathbb{P}\left((F / L)^{\vee}\right)$ on $\mathbb{P}\left(F^{\vee}\right)$, from the proof of Proposition 4.1.2, (ii) we know that the component of degree one of $\left(\widehat{c}\left(\bar{Q}_{F^{\vee}}\right) \mid I_{*}(Z)\right)$ in $\widehat{C H}^{*}(S)_{\mathbb{Q}}$ is equal to $\widehat{c}_{1}\left(\bar{F}^{\vee}\right)-\widehat{c}_{1}\left((\bar{F} / \bar{L})^{\vee}\right)=\widehat{c}_{1}\left(\bar{L}^{\vee}\right)$. It follows that $a(\lambda)=0$.

Proof of Theorem 4.3.2. Using successively Lemma 4.2.1, (2.3.13), (2.3.14), Proposition 4.3.3 and Proposition 2.3.1, (iii), we get

$$
\begin{aligned}
h\left(\mathrm{Ch}_{\mathbf{d}}(Z)\right) & =\widehat{\operatorname{deg}}\left(\prod_{i=0}^{r} \widehat{c}\left(\bar{Q}_{i}\right) \mid \Gamma_{*} v_{*}(Z)\right) \\
& =\widehat{\operatorname{deg}}\left(\Gamma^{*}\left(\prod_{i=0}^{r} \widehat{c}\left(\bar{Q}_{i}\right)\right) \mid v_{*}(Z)\right) \\
& =\widehat{\operatorname{deg}}\left(\prod_{i=0}^{r} \widehat{c}\left(\bar{L}_{i}\right) \mid v_{*}(Z)\right) \\
& =\widehat{\operatorname{deg}}\left(\prod_{i=0}^{r} \widehat{c}\left(v_{i}^{*}\left(\bar{L}_{i}\right)\right) \mid Z\right),
\end{aligned}
$$

where $\bar{L}_{i}$ is the canonical hermitian line bundle of degree one on $\mathbb{P}_{i}$. The Veronese embedding $v_{i}: \mathbb{P} \rightarrow \mathbb{P}_{i}$ is such that $v_{i}^{*}\left(\bar{L}_{i}\right)$ is canonically isomorphic to $\overline{\mathscr{O}\left(d_{i}\right)}$. Moreover, Chern classes of line bundles vanish in degree $>1$. Therefore we get:

$$
\begin{aligned}
h\left(\mathrm{Ch}_{\mathrm{d}}(Z)\right) & =\widehat{\operatorname{deg}}\left(\prod_{i=0}^{r} \widehat{c}_{1}\left(\overline{\mathscr{O}\left(d_{i}\right)}\right) \mid Z\right) \\
& =\prod_{i=0}^{r} d_{i} \cdot \widehat{\operatorname{deg}}\left(\widehat{c}_{1}(\overline{\mathscr{O}(1)}) ; Z\right) \\
& =\prod_{i=0}^{r} d_{i} \cdot h_{\overline{\mathscr{O}}(1)}(Z),
\end{aligned}
$$

which proves (i). To compute $\operatorname{deg}_{i}(Z)$ we use Lemma 4.2.1 to get

where

$$
\operatorname{deg}_{i}\left(\mathrm{Ch}_{\mathbf{d}}(Z)\right)=\operatorname{deg}_{K}\left(\Gamma^{*}(\alpha) \mid v_{*}(Z)\right),
$$

$$
\alpha=\widehat{c}_{N_{i}-1}\left(\bar{Q}_{i}\right) \prod_{\substack{j=0 \\ j \neq i}} \widehat{c}_{N_{j}}\left(\bar{Q}_{j}\right)
$$


From Proposition 4.3.3 we know that

$$
I_{j}^{*}\left(\widehat{c}_{N_{j}}\left(\bar{Q}_{j}\right)\right)=\widehat{c}_{1}\left(\bar{L}_{j}\right)
$$

and

$$
I_{i}^{*}\left(\widehat{c}_{N_{i}-1}\left(\bar{Q}_{i}\right)\right)=1
$$

Therefore

$$
\Gamma^{*}(\alpha)=\prod_{\substack{j=0 \\ j \neq i}}^{r} \widehat{c}_{1}\left(\bar{L}_{j}\right)
$$

and

$$
\begin{aligned}
\operatorname{deg}_{i}\left(\mathrm{Ch}_{\mathbf{d}}(Z)\right) & =\operatorname{deg}_{K}\left(v^{*}\left(\prod_{\substack{j=0 \\
j \neq i}}^{r} c_{1}\left(L_{j}\right)\right) \mid Z\right) \\
& =\left(\prod_{\substack{j=0 \\
j \neq i}}^{r} d_{j}\right) \cdot \operatorname{deg}_{K}(Z) .
\end{aligned}
$$

\subsubsection{The height of generalized Chow forms.}

4.3.4.1. We keep the notations of the previous subsection. Let $\bar{M}_{i}$ be the pullback to $\prod_{j=0}^{r} \stackrel{\vee}{\mathbb{P}}_{i}$ of the canonical hermitian line bundle $\overline{\mathscr{O}}_{S^{d i} \frac{i}{E}}(1)$ on $\stackrel{\vee}{\mathbb{P}}_{i}$, and $\mu_{i}=c_{1}\left(\bar{M}_{i}\right)$.

Lemma 4.3.4. For any $(r+1)$-tuple of integers $\left(\delta_{0}, \ldots, \delta_{r}\right)$ and any nonzero rational section $s$ of $\otimes_{i=1}^{r} M_{i}^{\otimes \delta_{i}}$ on $\prod_{i=0}^{r} \stackrel{\vee}{\mathbb{P}}_{i}$, the following formulae hold:

$$
h(\operatorname{div}(s))=\widehat{\operatorname{deg}}\left(\prod_{i=0}^{r} \widehat{c}_{1}\left(\bar{M}_{i}\right)^{N_{i}} \mid \operatorname{div}(s)\right)+\sum_{i=0}^{r} \delta_{i}\left(\widehat{\operatorname{deg}} S^{d_{i}} \frac{\vee}{E}-[K: \mathbb{Q}] \sigma_{N_{i}-1}\right)
$$

$$
=\sum_{\sigma: K \hookrightarrow \mathbb{C}} \int\left(\prod_{i=0}^{r} \begin{array}{l}
\mathbb{P}_{i} \\
)_{\sigma}(\mathbb{C})
\end{array} \log \|s\| \cdot \prod_{i=0}^{r} \mu_{i}^{N_{i}}+\frac{1}{2}[K: \mathbb{Q}] \sum_{i=0}^{r} \delta_{i}\left(1+\frac{1}{2}+\cdots+\frac{1}{N_{i}}\right) .\right.
$$

Proof. According to (4.1.7), we have:

$$
\widehat{c}_{N_{i}}\left(\bar{Q}_{i}\right)=\widehat{c}_{1}\left(\bar{M}_{i}\right)^{N_{i}}+\widehat{c}_{1}\left(\bar{M}_{i}\right)^{N_{i}-1} \pi^{*} \widehat{c}_{1}\left(S^{d_{i}} \frac{\vee}{E}\right)-2 a\left(\sigma_{N_{i}-1} \mu_{i}^{N_{i}-1}\right) .
$$


Therefore

$$
\begin{aligned}
\prod_{i=0}^{r} \widehat{c}_{N_{i}}\left(\bar{Q}_{i}\right) & =\prod_{i=0}^{r} \widehat{c}_{1}\left(\bar{M}_{i}\right)^{N_{i}}+\sum_{i=0}^{r}\left(\prod_{\substack{j=0 \\
\neq i \\
\text { in }}}^{r} \widehat{c}_{1}\left(\bar{M}_{j}\right)^{N_{j}}\right) \widehat{c}_{1}\left(\bar{M}_{i}\right)^{N_{i}-1} \pi^{*} \widehat{c}_{1}\left(S^{d_{i}} \frac{\vee}{E}\right) \\
& -2 a\left(\sum_{i=0}^{r} \sigma_{N_{i}-1}\left(\prod_{\substack{j=0 \\
\neq i}}^{r} \mu_{j}^{N_{j}}\right) \mu_{i}^{N_{i}-1}\right) .
\end{aligned}
$$

From (2.3.8), we get:

$$
\begin{aligned}
\widehat{\operatorname{deg}}\left(\prod_{i=0}^{r} \widehat{c}_{1}\left(\bar{M}_{i}\right)^{N_{i}} \mid \operatorname{div}(s)\right)= & \int\left(\prod_{i=0}^{r}{\left.\stackrel{\vee}{\mathbb{P}_{i}}\right)}_{(\mathbb{C})} \log \|s\| \cdot \prod_{i=0}^{r} \mu_{i}^{N_{i}}\right. \\
& +\widehat{\operatorname{deg}}\left(\prod_{i=0}^{r} \widehat{c}_{1}\left(\bar{M}_{i}\right)^{N_{i}} \cdot \widehat{c}_{1}\left(\stackrel{r}{\otimes}_{i=0}^{r} \bar{M}_{i}^{\otimes \delta_{i}}\right) \mid \prod_{i=0}^{r} \stackrel{\vee}{\mathbb{P}}_{i}\right) .
\end{aligned}
$$

Using (2.3.12) and (4.1.4), we obtain

$$
\begin{aligned}
& \widehat{\operatorname{deg}}\left(\prod_{i=0}^{r} \widehat{c}_{1}\left(\bar{M}_{i}\right)^{N_{i}} \cdot \widehat{c}_{1}\left(\underset{i=0}{\stackrel{r}{\otimes}} \bar{M}_{i}^{\otimes \delta_{i}}\right) \mid \prod_{i=0}^{r} \stackrel{\vee}{\mathbb{P}}_{i}\right) \\
& =\sum_{i=0}^{r} \delta_{i} \widehat{\operatorname{deg}}\left(\widehat{c}_{1}\left(\frac{}{\widehat{\mathscr{O}}_{S^{d_{i}} \vee}(1)}\right)^{N_{i}+1} \mid \stackrel{\vee}{\mathbb{P}}_{i}\right) \\
& =\sum_{i=0}^{r} \delta_{i}\left(-\widehat{\operatorname{deg}}\left(S^{d_{i}} \frac{v}{E}\right)+[K: \mathbb{Q}] \sigma_{N_{i}}\right) \text {. }
\end{aligned}
$$

Equality (4.3.9) follows from (4.3.8), (4.3.10), and (4.3.11).

Let $Z \in Z_{r+1}(\mathbb{P}(E))$ be a nonzero effective cycle, and let, for $i=0, \ldots, r$,

$$
\delta_{i}=\left(\prod_{\substack{j=0 \\ j \neq i}}^{r} d_{j}\right) \cdot \operatorname{deg}_{K} Z
$$

According to (4.3.6), the divisor $\mathrm{Ch}_{\mathrm{d}}(Z)_{K}$ is the divisor of a nonzero multihomogeneous form $\Phi_{\mathrm{d}, Z_{K}}$ in

$$
H^{0}\left(\prod_{i=0}^{r} \stackrel{\vee}{\mathbb{P}_{i K}} ; \underset{i=0}{\otimes} M_{i, K}^{\otimes \delta_{i}}\right) \simeq \stackrel{r}{\otimes} S_{i=0}^{\delta_{i}}\left(\begin{array}{ll}
S^{d_{i}} & \stackrel{\vee}{E_{K}}
\end{array}\right)^{\vee} .
$$

This generalized Chow form $\Phi_{\mathbf{d}, Z_{K}}$ of $Z_{K}$ may be seen as a rational section of $\underset{j=0}{\otimes} M_{j}^{\otimes \delta_{j}}$ over $\prod_{j=0}^{r} \stackrel{\vee}{\mathbb{P}}_{j}$. As $\mathrm{Ch}_{\mathbf{d}}(Z)$ and $\operatorname{div} \Phi_{\mathbf{d}, Z_{K}}$ are divisors in $\prod_{j=0}^{r} \stackrel{\vee}{\mathbb{P}}_{j}$ which 
coincide on the generic fiber, we can write

$$
\mathrm{Ch}_{\mathbf{d}}(Z)=\operatorname{div}\left(\Phi_{\mathbf{d}, Z_{K}}\right)-\sum_{\wp} n_{\wp}\left(\prod_{i=0}^{r} \stackrel{\vee}{\mathbb{P}_{i}}\right)_{\mathbb{F}_{p}}
$$

where $\wp$ runs over the closed points of $S$, and where $n_{\wp}$ is an integer, which vanishes for almost every $\wp$.

If we combine this relation with (4.3.9), we get

$$
\begin{aligned}
h\left(\mathrm{Ch}_{\mathbf{d}}(Z)\right)= & \sum_{\sigma: K \rightarrow \mathbb{C}} \int_{\left(\prod_{i=0}^{r}{\stackrel{\vee}{\mathbf{P}_{i}}}_{)^{\prime}}(\mathbb{C})\right.} \log \left\|\Phi_{\mathbf{d}, z_{K}}\right\| \cdot \prod_{i=0}^{r} \mu_{i}^{N_{i}}-\sum_{\wp} n_{\wp} \log N_{\wp} \\
& +\frac{1}{2}\left(\prod_{i=0}^{r} d_{i}\right) \cdot \sum_{i=0}^{r} \frac{1}{d_{i}}\left(1+\frac{1}{2}+\cdots+\frac{1}{N_{i}}\right) \cdot[K: \mathbb{Q}] \operatorname{deg}_{K} Z .
\end{aligned}
$$

This formula is especially interesting in the following situations:

- The vector bundle $E$ is the trivial vector bundle $\mathscr{O}_{S}^{\oplus(N+1)}$, and $Z$ is a nonzero effective cycle flat over $S$; then $\Phi_{\mathbf{d}, Z_{K}}$ is a polynomial

$$
\sum_{\left|I_{i}\right|=\delta_{i}} a_{I_{0}, \ldots, I_{r}} U_{0}^{I_{0}} \cdots U_{r}^{I_{r}}
$$

in variables $\left(U_{0, K}\right)_{\substack{K \in \mathbb{N}^{N+1} \\|K|=d_{0}}}, \ldots,\left(U_{r, K}\right)_{\substack{K \in \mathbb{N}^{N+1} \\|K|=d_{r}}}$ which represent the coefficients of the "generic" homogeneous polynomials of degree $d_{0}, \ldots, d_{r}$ in $N+1$ variables, and since $\mathrm{Ch}_{\mathrm{d}}(Z)$ is a flat divisor (Lemma 4.3.1), the multiplicities $n_{\wp}$ are given by

$$
n_{\wp}=\min _{I_{0}, \ldots, I_{r}} v_{\wp}\left(a_{I_{0}, \ldots, I_{r}}\right)
$$

In particular, if $\bar{E}$ is the trivial hermitian vector bundle $\overline{\mathscr{O}}_{S}^{\oplus(N+1)}$ and $\mathbf{d}=$ $(1, \ldots, 1)$, the two first term in the right-hand side of $(4.3 .12)$ gives the height of $Z$ as defined in [P2].

- The class number of $K$ is one (e.g., $K=\mathbb{Q}$ ); then, as observed in 4.3.2, (iii) there exists a generalized Chow form over $\mathscr{\sigma}_{K}$, i.e., a nonzero element $\Phi_{\mathbf{d}, Z}$ of

$$
H^{0}\left(\prod_{i=0}^{r} \stackrel{\vee}{\mathbb{P}}_{i} ; \stackrel{\otimes}{\otimes}_{i=0}^{r} M_{i}^{\otimes \delta_{i}}\right) \simeq \stackrel{r}{\otimes} S_{i=0}^{\delta_{i}}\left(S^{d_{i}} \stackrel{\vee}{E}\right)^{\vee}
$$

such that

$$
\mathrm{Ch}_{\mathbf{d}}(Z)=\operatorname{div}\left(\Phi_{\mathbf{d}, Z}\right)
$$

and (4.3.12) holds with $\Phi_{\mathbf{d}, Z}$ in place of $\Phi_{\mathbf{d}, Z_{K}}$ and $n_{\wp}=0$. 
4.3.4.2. We keep the notations of the previous paragraphs. For any family $N=\left(N_{\sigma}\right)_{\sigma: K \rightarrow \mathbb{C}}$ of continuous functions

$$
N_{\sigma}: \stackrel{r}{\otimes} S_{i=0}^{\delta_{i}}\left(S^{d_{i}} \stackrel{\vee}{E}\right)_{\sigma}^{\vee} \rightarrow \mathbb{R}_{+}
$$

such that

$$
\begin{aligned}
& N_{\sigma}(v)=0 \Leftrightarrow v=0, \\
& N_{\sigma}(\lambda v)=|\lambda| N_{\sigma}(v),
\end{aligned}
$$

and

$$
N_{\bar{\sigma}}(\bar{v})=N_{\sigma}(v)
$$

we may define

$$
h_{N}\left(\mathrm{Ch}_{\mathbf{d}}(Z)\right):=\sum_{\sigma: K \hookrightarrow \mathbb{C}} \log N_{\sigma}\left(\Phi_{\mathbf{d}, Z_{K}}\right)-\sum_{\wp} n_{\wp} \log N_{\wp}
$$

(by the product formula it does not depend on the choice of $\Phi_{\mathbf{d}, Z_{K}}$ ).

The choice of $N$ is equivalent to the choice of a continuous $F_{\infty}$-invariant metric $\|$.$\| on the line bundle \mathscr{O}(1)$ on $\mathbb{P}\left(\underset{i=0}{\stackrel{r}{\otimes}} S^{\delta_{i}}\left(S^{d_{i}} \stackrel{\vee}{E}\right)^{\vee}\right)$; namely, the dual metric $\|\cdot\|^{\vee}$ on $\mathscr{O}(-1)$ is such that for any $v \in \underset{i=0}{\stackrel{r}{\otimes}} S^{\delta_{i}}\left(S^{d_{i}} \stackrel{\vee}{E}\right)_{\sigma}^{\vee}-\{0\}$, the norm $\|v\|^{\vee}$ of $v$ seen as an element of the fiber $\mathscr{O}(-1)_{[v]}$ is $N_{\sigma}(v)$. When $Z$ is flat over $S, h_{N}\left(\mathrm{Ch}_{\mathbf{d}}(Z)\right)$ is nothing else than the height of the point

$$
\left[\Phi_{\mathrm{d}, Z_{K}}\right] \in \mathbb{P}\left(\stackrel{r}{\underset{i}{\otimes}} S^{\delta_{i}}\left(S^{d_{i}} \stackrel{\vee}{E}\right)^{\vee}\right)(K)
$$

defined by the hermitian line bundle $(\mathscr{O}(1),\|\cdot\|)$.

There are several natural choices for the family $N$ :

- For any $p \in[0, \infty]$, we can consider $N_{p}=\left(\|\|_{\sigma, p}\right)_{\sigma: K \hookrightarrow \mathbb{C}}$, where $\|\cdot\|_{\sigma, p}$ is the (quasi-)norm on

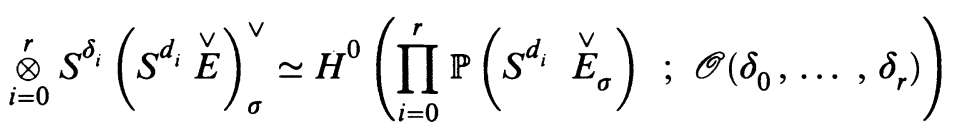

defined in 1.4.3, Remark (i) (as before, one uses the hermitian structure on $S^{d_{i}} \stackrel{\vee}{E}{ }_{\sigma}$ deduced from the one on $E$ ). We shall write $h_{p}$ instead of $h_{N_{p}}$.

- The hermitian structure on $E$ defines hermitian structures on $\left(S^{d_{i}} \stackrel{\vee}{E}\right)^{\vee}$ (see beginning of 4.3.3.1), hence on $\left(S^{d_{i}} \stackrel{\vee}{E}\right)^{\vee \otimes \delta_{i}}$, and, by considering the quotient metrics, on $S^{\delta_{i}}\left(S^{d_{i}} \stackrel{\vee}{E}\right)^{\vee}$. Finally, we get an hermitian structure on 
$\stackrel{r}{\otimes} S_{i=0}^{\delta_{i}}\left(S^{d_{i}} \stackrel{\vee}{E}\right)^{\vee}$, and we can take as $N$ the family $\left(\|\|_{\sigma}\right)_{\sigma: K \rightarrow \mathbb{C}}$ of hermitian norms defined by this hermitian structure. The height $h_{N}$ attached to this family will be denoted $h_{\mathrm{Herm}}$; see also, in the case $r=1$, [B-B-E-M].

- Let $\mathscr{B}=\left(e_{0}, \ldots, e_{N}\right)$ be any basis of $E_{K}$. From $\mathscr{B}$, we get the dual basis $\left(f_{0}, \ldots, f_{N}\right)$ of $\stackrel{\vee}{E_{K}}$, and a basis of $S^{d_{i}} \stackrel{\vee}{E}_{K}$, by forming the monomial $f^{I},|I|=d_{i}$; then, we consider the dual basis-it is a basis of $\left(\begin{array}{ll}S^{d_{i}} & \stackrel{\vee}{E_{K}}\end{array}\right)^{\vee}$ and finally the basis $\left(\Phi_{\lambda}\right)$ of $\stackrel{r}{\otimes} S_{i=0}^{\delta_{i}}\left(\begin{array}{ll}S^{d_{i}} & \stackrel{\vee}{E_{K}}\end{array}\right)^{\vee}$, obtained by taking products of monomials in the elements of these dual bases. We may consider the family of norms $N_{\mathscr{B}}=\left(N_{\mathscr{B}, \sigma}\right)_{\sigma: K \rightarrow \mathbb{C}}$ defined by

$$
N_{\mathscr{B}, \sigma}\left(\sum_{\lambda} a_{\lambda} \Phi_{\lambda, \sigma}\right)=\sum_{\lambda}\left|a_{\lambda}\right| \text {. }
$$

For instance, when $E_{K}=K^{N+1}$ and $\mathscr{B}$ is the standard basis, defined by $e_{i}=\left(\delta_{i j}\right)_{0 \leq j \leq N}$, then $\underset{i=0}{\otimes} S^{\delta_{i}}\left(\begin{array}{ll}S^{d_{i}} & \stackrel{\vee}{E_{K}}\end{array}\right)^{\vee}$ is the space of multihomogeneous polynomials of the form (4.3.13), the basis attached to $\mathscr{B}$ is formed by the monomials $U_{0}^{I_{0}} \cdots U_{r}^{I_{r}}\left(\left|I_{i}\right|=\delta_{i}\right)$, and

$$
N_{\mathscr{B}, \sigma}\left(\sum_{I_{0}, \ldots, I_{n}} a_{I_{0}, \ldots, I_{r}} U_{0}^{I_{0}} \cdots U_{r}^{I_{r}}\right)=\sum_{I_{0}, \ldots, I_{r}}\left|a_{I_{0}, \ldots, I_{r}}\right| .
$$

We shall write $h_{\mathscr{B}}$ instead of $h_{N_{\mathscr{B}}}$.

The heights $h_{p}, h_{\mathrm{Herm}}, h_{\mathscr{B}}$ may be compared as follows:

Proposition 4.3.5. Let $Z$ be any effective cycle in $Z_{r+1}(\mathbb{P}(E))$.

(i) For any $p \in[0, \infty]$, we have:

$$
h_{0}\left(\mathrm{Ch}_{\mathbf{d}}(Z)\right) \leq h_{p}\left(\mathrm{Ch}_{\mathbf{d}}(Z)\right) \leq h_{\infty}\left(\mathrm{Ch}_{\mathbf{d}}(Z)\right)
$$

Moreover:

$$
\begin{aligned}
h_{\infty}\left(\mathrm{Ch}_{\mathbf{d}}(Z)\right) \leq & h_{0}\left(\mathrm{Ch}_{\mathbf{d}}(Z)\right)+\frac{1}{2}\left(\prod_{i=0}^{r} d_{i}\right) \\
& \cdot \sum_{i=0}^{r} \frac{1}{d_{i}}\left(1+\frac{1}{2}+\cdots+\frac{1}{N_{i}}\right) \cdot[K: \mathbb{Q}] \operatorname{deg}_{K}(Z) .
\end{aligned}
$$

(ii) The following equality holds:

(4.3.18) $h_{\mathrm{Herm}}\left(\mathrm{Ch}_{\mathbf{d}}(Z)\right)=h_{2}\left(\mathrm{Ch}_{\mathbf{d}}(Z)\right)+\frac{1}{2}[K: \mathbb{Q}] \operatorname{deg}_{K}(Z) \sum_{i=0}^{r} \log \left(\begin{array}{c}N_{i}+\delta_{i} \\ \delta_{i}\end{array}\right)$. 
(iii) For any $(d, n) \in \mathbb{N}^{*} \times \mathbb{N}^{*}$, let

$$
m(d, n)=\min _{\substack{I \in \mathbb{N}^{n} \\|I|=d}} \frac{I !}{d !}
$$

and

$$
M(d, n)=\sum_{\substack{I \in \mathbb{N}^{n} \\|I|=d}} \frac{I !}{n !} .
$$

Then, for any basis $\mathscr{B}$ of $E_{K}$ which is orthonormal in $\bar{E}_{\sigma}$ for each $\sigma: K \rightarrow \mathbb{C}$, we have:

$$
\begin{aligned}
\frac{1}{2}\left(\prod_{i=0}^{r} d_{i}\right) \cdot \sum_{i=0}^{r} \frac{1}{d_{i}} \log m\left(d_{i}, N+1\right) \cdot[K: \mathbb{Q}] \operatorname{deg}_{K}(Z) \\
\quad \leq h_{\mathscr{B}}\left(C h_{\mathrm{d}}(Z)\right)-h_{\mathrm{Herm}}\left(C h_{\mathrm{d}}(Z)\right) \\
\quad \leq \frac{1}{2}\left(\prod_{i=0}^{r} d_{i}\right) \cdot \sum_{i=0}^{r} \frac{1}{d_{i}} \log M\left(d_{i}, N+1\right) \cdot[K: \mathbb{Q}] \operatorname{deg}_{K}(Z) .
\end{aligned}
$$

Inequalities (4.3.16) and (4.3.17) follow from 1.4.3, (ii), and Corollary 1.4.3. Equality (4.3.18) follows from the relations:

$$
\|\cdot\|_{\sigma}^{2}=\prod_{i=0}^{r}\left(\begin{array}{c}
N_{i}+\delta_{i} \\
\delta_{i}
\end{array}\right) \cdot\|\cdot\|_{2, \sigma}^{2} .
$$

By tensor product, this is a consequence of the following lemma (compare [GS6], p.537):

Lemma 4.3.6. Let $\bar{V}$ be any hermitian vector space of dimension $N+1$. For any $d \in \mathbb{N}$, the norm $\|$.$\| on S^{d} \stackrel{\vee}{V}$ defined by the hermitian structure on $S^{d} \frac{\vee}{V}$ and the norm $\|.\|_{2}$ on $S^{d} \stackrel{\vee}{V} \simeq H^{0}(\mathbb{P}(V) ; \mathscr{O}(d))$ defined in 1.4 .3 , (ii), are related by

$$
\|x\|^{2}=\left(\begin{array}{c}
N+d \\
d
\end{array}\right)\|x\|_{2}^{2}
$$

Proof of Lemma 4.3.6. The irreducibility of the action of the unitary group $U(\bar{V})$ on $S^{n} \stackrel{V}{V}$ implies the existence of $\lambda \in \mathbb{R}_{+}^{*}$ such that, for any $x \in S^{n} \stackrel{\vee}{V}$ :

$$
\|x\|^{2}=\lambda\|x\|_{2}^{2}
$$

To compute the constant $\lambda$, we may assume that $\bar{V}$ is $\mathbb{C}^{N+1}$ endowed with the standard hermitian scalar product and use the notations of 1.4.3. According to (4.3.20), we have for every multi-index $I \in \mathbb{N}^{N+1}$ of length $d$ :

$$
\frac{I !}{d !}=\lambda \int_{\mathbf{S}^{2 N+1}} z^{I} \frac{1}{z}^{I} d v
$$


Therefore

$$
\begin{aligned}
\lambda & =\lambda \int_{\mathbb{S}^{2 N+1}}\left(\sum_{i=0}^{r}\left|z_{i}\right|^{2}\right)^{d} d v \\
& =\lambda \sum_{\substack{I \in \mathbb{N}^{N+1} \\
|I|=d}} \frac{d !}{I !} \int_{\mathbb{S}^{2 N+1}} z^{I} \frac{z}{z}^{I} d v \\
& =\sum_{\substack{|I| \in \mathbb{N}^{N+1} \\
|I|=d}} 1=\left(\begin{array}{c}
N+d \\
d
\end{array}\right) \cdot
\end{aligned}
$$

To prove (4.3.19), we need to introduce a few notations. If $\mathscr{B}=\left(e_{1}, \ldots, e_{n}\right)$ is a basis of a finite-dimensional complex vector space $V$, we denote by \|\|$_{\mathscr{B}}$ the norm on $V$ defined by

$$
\left\|\sum_{i=1}^{n} x_{i} e_{i}\right\|=\sum_{i=1}^{n}\left|x_{i}\right|,
$$

we denote by $\stackrel{\vee}{\mathscr{B}}$ the dual basis of $\stackrel{\vee}{E}$, and, for any $d \in \mathbb{N}^{*}$, by $S^{d} \mathscr{B}$ the basis of $S^{d} E$ formed by monomials in the $e_{i}$ 's. If $\mathscr{B}_{1}=\left(e_{1}^{1}, \ldots, e_{n_{1}}^{1}\right), \ldots, \mathscr{B}_{k}=$ $\left(e_{1}^{k}, \ldots, e_{n_{k}}^{k}\right)$ are bases of complex vector spaces $V_{1}, \ldots, V_{k}$, we denote by $\mathscr{B}_{1} \otimes \cdots \otimes \mathscr{B}_{k}$ the basis $\left(e_{i_{1}}^{1} \otimes \cdots \otimes e_{i_{k}}^{d}\right){\substack{1 \leq i_{1} \leq n_{1} \\ 1 \leq i_{k} \leq n_{k}}}_{\substack{1 \\ n_{1}}}$ of $V_{1} \otimes \ldots \otimes V_{k}$. Finally, if $\bar{V}$ is any finite-dimensional hermitian vector space and $\mathscr{B}$ a basis of $V$, we let

$$
m(\bar{V}, \mathscr{B})=\min _{x \in V-\{0\}} \frac{\|x\|_{\mathscr{B}}^{2}}{\langle x, x\rangle}
$$

and

$$
M(\bar{V}, \mathscr{B})=\max _{x \in V-\{0\}} \frac{\|x\|_{\mathscr{B}}^{2}}{\langle x, x\rangle} .
$$

Lemma 4.3.7. Let $\mathscr{B}=\left(e_{1}, \ldots, e_{n}\right)$ be any orthogonal basis of an hermitian vector space $\bar{V}$.

(i) We have:

$$
m(\bar{V}, \mathscr{B})=\min _{1 \leq i \leq n}\left(\left\langle e_{i}, e_{i}\right\rangle^{-1}\right)
$$

and

$$
M(\bar{V}, \mathscr{B})=\sum_{1 \leq i \leq n}\left\langle e_{i}, e_{i}\right\rangle^{-1}
$$

(ii) For any $d \in \mathbb{N}^{*}, S^{d} \mathscr{B}$ is an orthogonal basis of $S^{d} \bar{V}$, and we have

$$
m\left(S^{d} \bar{V}, S^{d} \mathscr{B}\right)=m(\bar{V}, \mathscr{B})^{d}
$$


and

$$
M\left(S^{d} \bar{V}, S^{d} \mathscr{B}\right)=M(\bar{V}, \mathscr{B})^{d}
$$

(iii) If $\mathscr{B}$ is an orthonormal basis of $\bar{V}$, then for any $d \in \mathbb{N}^{*},\left(S^{d} \stackrel{\vee}{\mathscr{B}}\right)^{\vee}$ is an orthogonal basis of $\left(S^{d} \frac{\vee}{V}\right)^{\vee}$, and

$$
m\left(\left(S^{d} \frac{\vee}{V}\right)^{\vee},\left(S^{d} \stackrel{\vee}{\mathscr{B}}\right)^{\vee}\right)=m(d, n):=\min _{\substack{I \in N^{n} \\|I|=d}} \frac{I !}{d !}
$$

and

$$
M\left(\left(S^{d} \frac{\vee}{V}\right)^{\vee},\left(S^{d} \stackrel{\vee}{\mathscr{B}}\right)^{\vee}\right)=M(d, n):=\sum_{\substack{I \in \mathbb{N}^{n} \\|I|=d}} \frac{I !}{d !}
$$

(iv) If $\mathscr{B}^{\prime}$ is any orthogonal basis of a finite-dimensional hermitian vector space $\bar{V}^{\prime}$, then $\mathscr{B} \otimes \mathscr{B}^{\prime}$ is an orthogonal basis of $\bar{V} \otimes \bar{V}^{\prime}$, and we have

$$
m\left(\bar{V} \otimes \bar{V}^{\prime}, \mathscr{B} \otimes \mathscr{B}^{\prime}\right)=m(\bar{V}, \mathscr{B}) m\left(\bar{V}^{\prime}, \mathscr{B}^{\prime}\right)
$$

and

$$
M\left(\bar{V} \otimes \bar{V}^{\prime}, \mathscr{B} \otimes \mathscr{B}^{\prime}\right)=M(\bar{V}, \mathscr{B}) M\left(\bar{V}^{\prime}, \mathscr{B}^{\prime}\right) .
$$

Proof of Lemma 4.3.7. Equations (4.3.21) and (4.3.22) follow respectively from the triangle and Cauchy-Schwarz inequalities. Assertion (iv) immediately follows from (4.3.21) and (4.3.22).

To prove (ii), observe that $S^{d} \mathscr{B}=\left(e^{I}\right)_{\substack{I \in \mathbb{N}^{n} \\|I|=d}}$ and that, if we let $\alpha_{i}=\left\langle e_{i}, e_{i}\right\rangle$, then

$$
\left\langle e^{I}, e^{I}\right\rangle=\frac{I !}{d !} \alpha^{I}
$$

As $\frac{d !}{I !} \geq 1$ and $\frac{d !}{I !}=1$ if $I$ takes the form $\left(d \delta_{i j}\right)_{1 \leq j \leq n}$, this implies (4.3.23). Moreover, using the multinomial formula, we get:

$$
\sum_{\substack{I \in N^{n} \\|I|=d}}\left\langle e^{I}, e^{I}\right\rangle^{-1}=\sum_{\substack{I \in \mathbb{N}^{n} \\|I|=d}} \frac{d !}{I !} \alpha^{-I}=\left(\sum_{i=1}^{n} \alpha_{i}^{-1}\right)^{d}=M(\bar{V}, \mathscr{B})^{d}
$$

that is, (4.3.24).

Let us prove assertion (iii). Let $\stackrel{\vee}{\mathscr{B}}=\left(f_{1}, \ldots, f_{n}\right)$; then $S^{d} \stackrel{\vee}{\mathscr{B}}=\left(f^{I}\right)_{\substack{i \in \mathbb{N}^{n} \\|I|=d}}$, and $\left(S^{d} \stackrel{\vee}{\mathscr{B}}\right)^{\vee}=\left(E_{I}\right)_{\substack{I \in \mathbb{N}^{n} \\|I|=d}}$, where $E_{I}$ is defined by

$$
E_{I}\left(f^{J}\right)=\delta_{I, J} .
$$


The bases $\stackrel{\vee}{\mathscr{B}}, S^{d} \stackrel{\vee}{\mathscr{B}}$, and $\left(S^{d} \stackrel{\vee}{\mathscr{B}}\right)^{\vee}$ are orthogonal. Moreover, the equality above shows that

$$
\left\langle E_{I}, E_{I}\right\rangle^{-1}=\left\langle f^{I}, f^{I}\right\rangle
$$

and, since $\stackrel{\vee}{\mathscr{B}}$ is orthonormal,

$$
\left\langle f^{I}, f^{I}\right\rangle=\frac{I !}{d !}
$$

This proves (4.3.25) and (4.3.26).

From Lemma 4.3.7, we get that, under the hypotheses of (iii),

$$
\prod_{i=0}^{r} m\left(d_{i}, N+1\right)^{\delta_{i}} \leq \frac{N_{\mathscr{B}, \sigma}^{2}}{\|\cdot\|_{\sigma}^{2}} \leq \prod_{i=0}^{r} M\left(d_{i}, N+1\right)^{\delta_{i}} .
$$

This implies (4.3.19).

4.3.4.3. Finally, by combining the results of the preceding subsections, we can prove:

Theorem 4.3.8. For any effective cycle $Z \in Z_{r+1}(\mathbb{P}(E))$, we have:

$$
\begin{aligned}
h_{0}\left(\mathrm{Ch}_{\mathbf{d}}(Z)\right)= & \left(\prod_{i=0}^{r} d_{i}\right) \cdot h_{\overline{\mathscr{O}}(1)}(Z)-\frac{1}{2}\left(\prod_{i=0}^{r} d_{i}\right) \\
& \cdot \sum_{i=0}^{r} \frac{1}{d_{i}}\left(1+\frac{1}{2}+\cdots+\frac{1}{N_{i}}\right)[K: \mathbb{Q}] \operatorname{deg}_{K}(Z)
\end{aligned}
$$

and

$$
h_{\mathrm{Herm}}\left(\mathrm{Ch}_{\mathbf{d}}(Z)\right)=\left(\prod_{i=0}^{r} d_{i}\right) \cdot h_{\overline{\mathscr{O}(1)}}(Z)+[K: \mathbb{Q}] \operatorname{deg}_{K}(Z) \varepsilon_{\mathbf{d}}(Z) \text {, }
$$

where

$$
\left|\varepsilon_{\mathrm{d}}(Z)\right| \leq \frac{1}{2}\left(\prod_{i=0}^{r} d_{i}\right) \cdot \sum_{i=0}^{r} \frac{1}{d_{i}} \log \left(N_{i}+1\right)
$$

Moreover, for any basis $\mathscr{B}$ of $E_{K}$ which is orthonormal in $\bar{E}_{\sigma}$ for each embedding $\sigma: K \rightarrow \mathbb{C}$, we have:

$$
h_{\mathscr{B}}\left(\mathrm{Ch}_{\mathbf{d}}(Z)\right)=\left(\prod_{i=0}^{r} d_{i}\right) \cdot h_{\overline{\mathscr{O}}(\mathbf{1})}(Z)+[K: \mathbb{Q}] \operatorname{deg}_{K}(Z) \eta_{\mathbf{d}}(Z) \text {, }
$$


where

$$
\begin{gathered}
-\frac{1}{2}\left(\prod_{i=0}^{r} d_{i}\right) \cdot\left[(r+1) \cdot \log (N+1)+\sum_{i=0}^{r} \frac{1}{d_{i}} \log \left(N_{i}+1\right)\right] \\
\leq \eta_{\mathrm{d}}(Z) \leq\left(\prod_{i=0}^{r} d_{i}\right) \cdot \sum_{i=0}^{r} \frac{1}{d_{i}} \log \left(N_{i}+1\right)
\end{gathered}
$$

Observe that

$$
N_{i}+1=\left(\begin{array}{c}
N+d_{i} \\
d_{i}
\end{array}\right) \leq\left(d_{i}+1\right)^{N}
$$

Therefore we get the following upper bound for the right-hand side in (4.3.30) and (4.3.32):

$$
\prod_{i=0}^{r} d_{i} \cdot \sum_{i=0}^{r} \frac{1}{d_{i}} \log \left(N_{i}+1\right) \leq N .\left(\prod_{i=0}^{r} d_{i}\right) \cdot \sum_{i=0}^{r} \frac{1}{d_{i}} \log \left(d_{i}+1\right) .
$$

Proof of Theorem 4.3.8. Equation (4.3.28) follows from (4.3.5), (4.3.12), and the definition of $h_{0}$.

If we combine (4.3.28) and Proposition 4.3.5, we get:

$$
h_{\mathrm{Herm}}\left(C h_{\mathbf{d}}(Z)\right)=\left(\prod_{i=0}^{r} d_{i}\right) \cdot h_{\overline{\mathscr{O}(1)}}(Z)+[K: \mathbb{Q}] \widetilde{\varepsilon}_{\mathbf{d}}(Z),
$$

where

$$
\begin{aligned}
& -\frac{1}{2}\left(\prod_{i=0}^{r} d_{i}\right) \cdot \sum_{i=0}^{r} \frac{1}{d_{i}}\left(1+\frac{1}{2}+\cdots+\frac{1}{N_{i}}\right) \operatorname{deg}_{K}(Z)+\frac{1}{2} \sum_{i=0}^{r} \log \left(\begin{array}{c}
N_{i}+\delta_{i} \\
\delta_{i}
\end{array}\right) \\
& \leq \widetilde{\varepsilon}_{\mathrm{d}}(Z) \leq \frac{1}{2} \sum_{i=0}^{r} \log \left(\begin{array}{c}
N_{i}+\delta_{i} \\
\delta_{i}
\end{array}\right),
\end{aligned}
$$

and

$$
h_{\mathscr{B}}\left(C h_{\mathbf{d}}(Z)\right)=\prod_{i=0}^{r} d_{i} \cdot h_{\overline{\mathscr{O}}(1)}(Z)+[K: \mathbb{Q}] \widetilde{\eta}_{\mathbf{d}}(Z)
$$


where

(4.3.36)

$$
\begin{aligned}
\frac{1}{2}\left(\prod_{i=0}^{r} d_{i}\right) & \cdot \sum_{i=0}^{r} \frac{1}{d_{i}}\left(\operatorname{deg}_{K}(Z) \cdot\left(\log m\left(d_{i}, N+1\right)-1-\frac{1}{2}-\cdots-\frac{1}{N_{i}}\right)\right) \\
+ & \frac{1}{2} \sum_{i=0}^{r} \log \left(\begin{array}{c}
N_{i}+\delta_{i} \\
\delta_{i}
\end{array}\right) \\
\leq & \tilde{\eta}_{\mathrm{d}}(Z) \leq \frac{1}{2}\left(\prod_{i=0}^{r} d_{i}\right) \cdot \sum_{i=0}^{r} \frac{1}{d_{i}} \operatorname{deg}_{K}(Z) \cdot \log M\left(d_{i}, N+1\right) \\
+ & \frac{1}{2} \sum_{i=0}^{r} \log \left(\begin{array}{c}
N_{i}+\delta_{i} \\
\delta_{i}
\end{array}\right) .
\end{aligned}
$$

Inequality (4.3.30) (resp. (4.3.32)) follows from (4.3.34) (resp. (4.3.36)) together with the estimates

$$
\begin{gathered}
\log N_{i} \leq 1+\frac{1}{2}+\cdots+\frac{1}{N_{i}} \leq \log \left(N_{i}+1\right) \\
\left(\begin{array}{c}
N_{i}+\delta_{i} \\
\delta_{i}
\end{array}\right) \leq\left(N_{i}+1\right)^{\delta_{i}} \\
m\left(d_{i}, N+1\right) \geq(N+1)^{-d_{i}}
\end{gathered}
$$

and

$$
M\left(d_{i}, N+1\right) \leq N_{i}
$$

Notice that (4.3.37) follows from the multinomial identity

$$
\sum_{\substack{I \in N^{N+1} \\|I|=d_{i}}} \frac{d_{i} !}{I !}=(N+1)^{d_{i}},
$$

and (4.3.38) follows from the fact that there are $N_{i}$ terms in the sum defining $M\left(d_{i}, N+1\right)$, each of them being at most one.

4.3.4.4. If we apply Theorem 4.3 .8 to the case where $K=\mathbb{Q}, \bar{E}$ is the trivial hermitian vector bundle $\overline{\mathscr{O}}_{\operatorname{Spec}(\mathbb{Z})}^{\oplus(N+1)}, r=N$, and $Z=\mathbb{P}_{\mathbb{Z}}^{N}$, we get the formula for the size of the resultant $R_{\mathrm{d}}$ of $N+1$ homogeneous polynomials of degrees $d_{0}, \ldots, d_{N}$ in $N+1$ variables (cf. 4.3.2, (v)). Namely, using the expression (3.3.2) for the Faltings height of $\mathbb{P}_{\mathbb{Z}}^{N}$, Theorem 4.3.8, and (4.3.33), we get

$$
\begin{gathered}
\log \left\|R_{\mathrm{d}}\right\|_{0}=\left(\prod_{i=0}^{N} d_{i}\right) \cdot\left(\sigma_{N}-\frac{1}{2} \sum_{i=0}^{N} \frac{1}{d_{i}}\left(1+\frac{1}{2}+\cdots+\frac{1}{N_{i}}\right)\right), \\
\left|\log \left\|R_{\mathrm{d}}\right\|_{\text {Herm }}-\left(\prod_{i=0}^{N} d_{i}\right) \cdot \sigma_{N}\right| \leq \frac{1}{2} N\left(\prod_{i=0}^{N} d_{i}\right) \cdot \sum_{i=0}^{N} \frac{1}{d_{i}} \log \left(d_{i}+1\right),
\end{gathered}
$$


and, if $\mathscr{B}$ is the standard basis of $\mathbb{Q}^{N+1}$,

$$
\log N_{\mathscr{B}}\left(R_{\mathrm{d}}\right) \leq\left(\prod_{i=0}^{N} d_{i}\right) \cdot\left(\sigma_{N}+N \sum_{i=0}^{N} \frac{1}{d_{i}} \log \left(d_{i}+1\right)\right) .
$$

Observe that $\left\|R_{\mathrm{d}}\right\|_{0}$ may be written concretely as follows: The resultant $R_{\mathrm{d}}$ is a multihomogeneous polynomial in

$$
\mathbb{C}\left[X_{0}, \ldots, X_{N}\right]_{d_{0}} \times \cdots \times \mathbb{C}\left[X_{0}, \ldots, X_{N}\right]_{d_{N}}
$$

let $\mathbb{S}^{2 N_{i}-1}$ be the unit sphere in $\mathbb{C}\left[X_{0}, \ldots, X_{N}\right]_{d_{i}}$ equipped with the hermitian norm $\|$.$\| defined by$

$$
\left\|\sum_{\substack{I \in N^{N+1} \\ I I \mid=d_{i}}} U_{I} X^{I}\right\|^{2}=\sum_{\substack{I \in N^{N+1} \\ I I \mid=d_{i}}} \frac{I !}{d_{i} !}\left|U_{I}\right|^{2},
$$

and let $d v_{i}$ be the unitary invariant probability measure on $\mathbb{S}^{2 N_{i}-1}$. Then:

$$
\log \left\|R_{\mathbf{d}}\right\|_{0}=\int_{\prod_{i=0}^{N} \mathbb{S}^{2 N_{i}-1}} \log \left|R_{\mathbf{d}}\right| d v_{0} \cdots d v_{N}
$$

In particular, when $d_{0}=\cdots=d_{N}=1, R$ is just the determinant of size $N+1$, and (4.3.39) reads

$$
\begin{aligned}
& \int_{\left(\mathbb{S}^{2 N+1}\right)^{N+1}} \log \left|\operatorname{det}\left(x_{i j}\right)_{0 \leq i, j \leq N}\right|^{2} d v \\
& \quad=2 \sigma_{N}-(N+1)\left(1+\frac{1}{2}+\cdots+\frac{1}{N}\right)=-N,
\end{aligned}
$$

where $\left(\mathbb{S}^{2 N+1}\right)^{N+1}$ denotes the product of spheres of equations $\sum_{i=0}^{N}\left|x_{i j}\right|^{2}=1$, $0 \leq j \leq N$, and $d v$ the product of the $U(N+1)$-invariant probability measures on these spheres.

Recall also that the norm $\left\|R_{\mathrm{d}}\right\|_{\mathscr{B}}$ which appears in (4.3.41) is nothing else than the sum of the absolute values of the coefficient of $R_{d}$ (cf. (4.3.15)). An estimate of this norm has already been obtained by Wüstholz ([Wu], Proposition 7) by using Macaulay's construction of $R_{d}$ as g.c.d. of generalized Sylvester's determinants; namely, he proves that

$$
\log \left\|R_{\mathbf{d}}\right\|_{\mathscr{B}} \leq\left(\begin{array}{c}
|\mathbf{d}|+1 \\
N
\end{array}\right)+\log \left(\begin{array}{c}
|\mathbf{d}|+1 \\
N
\end{array}\right) !
$$

where $|\mathbf{d}|=d_{0}+\cdots+d_{N}$. When the $d_{i}$ 's are bounded and $N$ goes to infinity, (4.3.41) is better than (4.3.43). On the other hand, when $N$ is fixed and $\mathbf{d}$ goes to infinity, the right-hand side of (4.3.43) grows like $|\mathbf{d}|^{N} \log |\mathbf{d}|$, and therefore (4.3.43) improves on (4.3.41). Observe also that, when $N$ is fixed, $\log \left\|R_{\mathrm{d}}\right\|_{p}$ and $\log \left\|R_{\mathrm{d}}\right\|_{\text {Herm }}$ are of the same order as $|\mathbf{d}|^{N+1}$, hence $R_{\mathrm{d}}$ is a form on which the ratios $\frac{\|\cdot\|_{p}}{\|\cdot\|_{\mathscr{B}}}$ and $\frac{\|\cdot\|_{\text {Herm }}}{\|.\|_{\mathscr{B}}}$ take "large" values. 
4.3.4.5. The computation of the height of Chow forms allows one to give another proof of the Finiteness Theorem 3.2.5.

Recall that to prove Theorem 3.2.5 it is enough to prove that when $\bar{E}$ is the trivial hermitian vector bundle $\overline{\mathscr{O}}_{S}^{\oplus(N+1)}$, then a set of flat effective cycles in $\mathbb{P}(E)=\mathbb{P}_{S}^{N}$ of bounded degrees and heights is finite. Consider the morphism

$$
f: \mathbb{P}_{S}^{N}=\mathbb{P}_{\mathbb{Z}}^{N} \times_{\mathbb{Z}} S \rightarrow \mathbb{P}_{\mathbb{Z}}^{N},
$$

defined as the projection onto the first factor. The map

$$
f_{*}=Z \cdot\left(\mathbb{P}_{S}^{N}\right) \rightarrow Z \cdot\left(\mathbb{P}_{\mathbb{Z}}^{N}\right),
$$

restricted to effective cycles, is finite to one. Together with the relations (3.1.7) and (3.1.9), this shows that to prove the required finiteness we may assume that $S=\operatorname{Spec}(\mathbb{Z})$.

Theorem 4.3.8 shows that the Chow form $\Phi_{Z}:=\Phi_{(1, \ldots, 1), Z}$ over $\mathbb{Z}$ of a flat effective cycle $Z$ in $Z_{r+1}\left(\mathbb{P}_{\mathbb{Z}}^{N}\right)$ of degree $\delta$ is a multihomogeneous polynomial with $\mathbb{Z}$-coefficients of multidegree $(\delta, \ldots, \delta)$, the norm of which is bounded in terms of $\delta$ and the height of $Z$. Therefore, the set of possible $\Phi_{Z}$ when the degree and the height of $Z$ are bounded is finite. As a flat cycle is determined by its Chow form, this completes the proof of Theorem 3.2.5.

Observe that this proof, contrary to the one in 3.2 .4 , does not rely on the special case $p=1$ of Theorem 3.2.5, that is, the finiteness of effective 0 -cycles in $\mathbb{P}_{\mathbb{Q}}^{N}$ of bounded degree and height. In fact, when $p=1$, our last proof coincides with the classical proof of this fact (see [No], p. 503 and [We2], p. 426; the homogeneous polynomials $\varphi$ and $F$ in [loc. cit.] are nothing else than Chow forms for 0 -cycles in $\mathbb{P}_{\mathbb{Q}}^{N}$, whose definition goes back at last to [Kro], §24).

It may be worth noting that the close relation between Chow forms and heights was already advocated in 1950 by Weil ([We1], p. 96), who claimed that it should be interpreted in a yet to be developed "geometry over integers", which would realize the program initiated by Kronecker's Grundzüge [Kro].

\section{Positivity OF THE PROJECTIVE heIGHT AND ARITHMETIC BÉZOUT THEOREM}

5.1. The basic inequality. Let $\mathbb{P}^{N}(\mathbb{C})=\mathbb{P}\left(\mathbb{C}^{N+1}\right)$ be the $N$-dimensional complex projective space, $\overline{\mathscr{O}(1)}$ the canonical line bundle of degree one on $\mathbb{P}^{N}(\mathbb{C})$ with its standard metric, $\mu=c_{1}(\overline{\mathscr{O}(1)})$ the Fubini-Study Kähler form, $W \subset$ $\mathbb{C}^{N+1}$ a complex linear subspace of dimension $q+1$ (with $0 \leq q \leq N$ ), and $\mathbb{P}(W) \subset \mathbb{P}^{N}(\mathbb{C})$ its projective space. Denote by $\Lambda$ the Levine form of $\mathbb{P}(W)$ in $\mathbb{P}_{\mathbb{C}}^{N}$ (see 1.2.3, Example $\left.(\mathrm{v})\right), H(\Lambda)$ its harmonic projection for the standard Kähler structure, and

$$
g_{\mathbb{P}(W)}=\Lambda-H(\Lambda) .
$$


Observe that $g_{\mathbb{P}(W)}$ is a $\mu$-normalized Green current for $\mathbb{P}(W)$. Let $W^{\perp}$ be the orthogonal complement of $W$ in $\mathbb{C}^{N+1}$ (for the standard scalar product) and

$$
f: \mathbb{P}^{N}(\mathbb{C})-\mathbb{P}\left(W^{\perp}\right) \rightarrow \mathbb{P}(W)
$$

the map induced by the orthogonal projection of $\mathbb{C}^{N+1}$ onto $W$. Finally, denote by $\operatorname{deg}(X)$ the degree of an algebraic cycle in $\mathbb{P}^{N}(\mathbb{C})$.

Proposition 5.1.1. Let $p$ be an integer such that $0 \leq p \leq N$ and $p+q \geq N$, and let $X \in Z_{p}\left(\mathbb{P}^{N}(\mathbb{C})\right)$ be an effective cycle on $\mathbb{P}^{N}(\mathbb{C})$ which meets $\mathbb{P}(W)$ properly. Then the following inequality holds:

$$
\frac{1}{2} \int_{\mathbb{P}^{N}(\mathbb{C})} \delta_{X} g_{\mathbb{P}(W)} \mu^{p+q+1-N} \geq \operatorname{deg}(X)\left(\sigma_{p}+\sigma_{q}-\sigma_{N}-\sigma_{p+q-N}\right) .
$$

Equality occurs if and only if there is an effective cycle $Y \subset \mathbb{P}(W)$ such that $X=\overline{f^{*}(Y)}$.

Observe that, as any hermitian vector space $V$ of dimension $N+1$ is isomorphic to $\mathbb{C}^{N+1}$ equipped with the standard metric, this proposition immediately extends to the situation where $\mathbb{P}^{N}(\mathbb{C})$ is replaced by $\mathbb{P}(V)$.

Proof. We may assume that $X$ is irreducible. For any $\lambda \in \mathbb{C}^{*}$, define an automorphism $\varphi_{\lambda}$ of $\mathbb{P}^{N}(\mathbb{C})$ by sending the class of $v \oplus w, v \in W, w \in W^{\perp}$, to the class of $\lambda v \oplus w$. Let $\Phi \subset \mathbb{A}^{1}(\mathbb{C}) \times \mathbb{P}^{N}(\mathbb{C})$ be the Zariski closure of the set of points $\left(\lambda, \varphi_{\lambda}(x)\right) \in \mathbb{C}^{*} \times \mathbb{P}^{N}(\mathbb{C}), x \in X(\mathbb{C})$. It $t$ is the standard coordinate of the affine line $\mathbb{A}^{1}(\mathbb{C})$, and $\lambda \in \mathbb{C}$, the cycle on $\mathbb{A}^{1}(\mathbb{C}) \times \mathbb{P}^{N}(\mathbb{C})$ defined by the divisor on $\Phi$ of the restriction of the function $t-\lambda$ takes the form $\{\lambda\} \times \Phi_{\lambda}$ for some effective cycle $\Phi_{\lambda}$ on $\mathbb{P}^{N}(\mathbb{C})$.

When $\lambda \neq 0, \Phi_{\lambda}$ is the irreducible cycle $\varphi_{\lambda}(X)$. Its specialization $\Phi_{0}$ when $\lambda \rightarrow 0$ coincides with the Zariski closure $\overline{f^{*}(X \cdot \mathbb{P}(W))}$ of the inverse image $f^{*}(X \cdot \mathbb{P}(W))$ in $\mathbb{P}^{N}(\mathbb{C})-\mathbb{P}\left(W^{\perp}\right)$ of the intersection cycle of $X$ and $\mathbb{P}(W)$. Indeed, $\mathbb{P}^{N}(\mathbb{C})-\mathbb{P}\left(W^{\perp}\right)$ may be canonically identified with the total space of the vector bundle $W^{\perp} \otimes \mathscr{O}(1)$ on $\mathbb{P}(W)$ (consider the map which sends the class $[v \oplus w],(v, w) \in W \times W^{\perp}$, to $([v], \lambda)$, where $\lambda: \mathbb{C} v \rightarrow W^{\perp}$ is the linear map defined by $\lambda(v)=w)$. Then $f$ gets identified with the projection map $W^{\perp} \otimes \mathscr{O}(1)_{\mid \mathbb{P}(W)} \rightarrow \mathbb{P}(W)$ and $\varphi_{\lambda}$ with the multiplication by $\lambda^{-1}$ in the fibers of $W^{\perp} \otimes \mathscr{O}(1)_{\mid \mathbb{P}(W)}$. The description of $X \cdot \mathbb{P}(W)$ using deformation to the normal cone of $\mathbb{P}(W)$ shows that

$$
f^{*}(X \cdot \mathbb{P}(W))=\Phi_{0 \mid \mathbf{P}^{N}(\mathbb{C})-\mathbf{P}\left(W^{\perp}\right)} .
$$

Moreover, since $\operatorname{dim} \Phi_{0}=p>\operatorname{dim} \mathbb{P}\left(W^{\perp}\right)=N-q-1$, we have

$$
\Phi_{0}=\overline{\Phi_{0 \mid \mathbb{P}^{N}(\mathbf{C})-\mathbb{P}\left(W^{\perp}\right)}}
$$


Let $\operatorname{pr}_{1}: \mathbb{A}^{1}(\mathbb{C}) \times \mathbb{P}^{N}(\mathbb{C}) \rightarrow \mathbb{A}^{1}(\mathbb{C})$ and $\operatorname{pr}_{2}: \mathbb{A}^{1}(\mathbb{C}) \times \mathbb{P}^{N}(\mathbb{C}) \rightarrow \mathbb{P}^{N}(\mathbb{C})$ be the two projections. For any $\lambda \in \mathbb{C}$ define

$$
\psi(\lambda)=\int_{\mathbf{P}^{N}(\mathbb{C})} \delta_{\Phi_{\lambda}} \cdot g_{\mathbf{P}(W)} \cdot \mu^{p+q+1-N} .
$$

According to Proposition 1.5.1, the function $\psi$ is continuous and the associated distribution $[\psi]$ coincides with

$$
\operatorname{pr}_{1 *}\left(\delta_{\Phi} \operatorname{pr}_{2}^{*}\left(g_{\mathbb{P}(W)} \mu^{p+q+1-N}\right)\right) .
$$

We shall now prove that, for all $\lambda \in \mathbb{C}$,

$$
\psi(\lambda) \geq \psi(0)
$$

Indeed, we deduce from the relation $d d^{c} g_{\mathbb{P}(W)}+\delta_{\mathbf{P}(W)}=\mu^{N-q}$ that

$$
d d^{c}\left(\delta_{\Phi} \operatorname{pr}_{2}^{*}\left(g_{\mathbb{P}(W)}\right)\right)+\delta_{\Phi \cdot \operatorname{pr}_{2}^{*}(\mathbb{P}(W))}=\delta_{\Phi} \operatorname{pr}_{2}^{*}\left(\mu^{N-q}\right),
$$

hence

$$
d d^{c}([\psi])=\operatorname{pr}_{1 *}\left(\delta_{\Phi} \operatorname{pr}_{2}^{*}\left(\mu^{p+1}\right)\right)-\operatorname{pr}_{1 *}\left(\delta_{\Phi \cdot \operatorname{pr}_{2}^{*}(\mathbb{P}(W))} \operatorname{pr}_{2}^{*}\left(\mu^{p+q+1-N}\right)\right) .
$$

Since $\Phi \cdot \operatorname{pr}_{2}^{*}(\mathbb{P}(W))=\operatorname{pr}_{2}^{*}(X \cdot \mathbb{P}(W))$, we have

$$
\operatorname{pr}_{1 *}\left(\delta_{\Phi \cdot p r_{2}^{*}(\mathbb{P}(W))} \operatorname{pr}_{2}^{*}\left(\mu^{p+q+1-N}\right)\right)=\operatorname{pr}_{1 *} \operatorname{pr}_{2}^{*}\left(\delta_{X \cdot \mathbb{P}(W)} \mu^{p+q+1-N}\right)=0
$$

for degree reasons. Therefore, the real current of type $(1,1)$

$$
d d^{c}([\psi])=\operatorname{pr}_{1 *}\left(\delta_{\Phi} \operatorname{pr}_{2}^{*}\left(\mu^{p+1}\right)\right)
$$

is positive on the complex line, in the sense that its integral on a positive function in $\mathscr{C}_{c}^{\infty}(\mathbb{C})$ is positive.

On the other hand, when $|\lambda|=1$ the automorphism $\varphi_{\lambda}$ is unitary. Since it fixes $\mathbb{P}(W)$, we see that $\varphi_{\lambda}^{*} g_{\mathbb{P}(W)}=g_{\mathbb{P}(W)}$, hence, for any $\lambda^{\prime} \in \mathbb{C}, \psi\left(\lambda \lambda^{\prime}\right)=$ $\psi\left(\lambda^{\prime}\right)$. In other words, $\psi(\lambda)$ is a continuous function of the norm $|\lambda|$ and we may find a continuous real function $\chi: \mathbb{R} \rightarrow \mathbb{R}$ such that

$$
\psi(\lambda)=\chi(\log |\lambda|) \text { when } \lambda \in \mathbb{C}^{*},
$$

and $\psi(0)=\lim _{x \rightarrow-\infty} \chi(x)$. Since $d d^{c}[\psi]$ is positive, the second derivative of $\chi$ is a nonnegative distribution. It follows that $\chi$ is convex, and therefore bounded below by $\psi(0)$. This proves $(5.1 .3)$.

To prove the inequality (5.1.2), it is now enough to show that $\psi(0) / 2$ coincides with the right-hand side of (5.1.2). Indeed, by the very definition of $\psi$, its left-hand side coincides with $\psi(1) / 2$ and, according to (5.1.3), $\psi(1) \geq \psi(0)$. 
Let $b: \widetilde{\mathbb{P}} \rightarrow \mathbb{P}^{N}(\mathbb{C})$ be the blow up of $\mathbb{P}^{N}(\mathbb{C})$ along $\mathbb{P}\left(W^{\perp}\right)$ and let $\tilde{f}: \widetilde{\mathbb{P}} \rightarrow$ $\mathbb{P}(W)$ be the map extending the projection $\mathbb{P}^{N}(\mathbb{C})-\mathbb{P}\left(W^{\perp}\right) \rightarrow \mathbb{P}(W)$. We get

$$
\begin{aligned}
\psi(0) & =\int_{\mathbb{P}^{N}(\mathbb{C})} \delta_{\overline{f^{*}(X \cdot \mathbb{P}(W))}} g_{\mathbb{P}(V)} \mu^{p+q+1-N} \\
& =\int_{\widetilde{\mathbb{P}}} \delta_{\widetilde{f}^{*}(X \cdot \mathbb{P}(W))} b^{*}\left(g_{\mathbb{P}(W)} \mu^{p+q+1-N}\right) \\
& =\int_{\mathbb{P}(W)} \delta_{X \cdot \mathbb{P}(W)} \widetilde{f}_{*} b^{*}\left(g_{\mathbb{P}(W)} \mu^{p+q+1-N}\right) .
\end{aligned}
$$

The unitary group $U(W)$ of $W$ for the induced metric acts upon $\mathbb{P}^{N}(\mathbb{C})$ by the decomposition $\mathbb{C}^{N+1}=W \oplus W^{\perp}$. This action extends to $\widetilde{\mathbb{P}}$, commutes with $b$ and $\tilde{f}$, fixes $g_{\mathbb{P}(W)}$ and $\mu$, and therefore $\tilde{f}_{*} b^{*}\left(g_{\mathbb{P}(W)} \mu^{p+q+1-N}\right)$ is $U(W)$-invariant, hence harmonic on $\mathbb{P}(W)$. If $\mu_{W}$ is the restriction of $\mu$ to $\mathbb{P}(W)$ we have

$$
\tilde{f}_{*} b^{*}\left(g_{\mathbb{P}(W)} \mu^{p+q+1-N}\right)=\alpha \mu_{W}^{p+q-N},
$$

where $\alpha$ is a real constant depending only on $p, q$, and $N$. It follows that

$$
\psi(0)=\alpha \operatorname{deg}(X) .
$$

To compute $\alpha$ let $W^{\prime} \subset W$ be a subspace of dimension $p+q+1-N$ and $X=\mathbb{P}\left(W^{\prime} \oplus W^{\perp}\right)$. In that case $\Phi_{0}=X$, therefore

$$
\psi(0)=\alpha=\int_{\mathbb{P}\left(W^{\prime} \oplus W^{\perp}\right)} g_{\mathbb{P}(W)} \mu^{p+q+1-N} .
$$

The restriction of the Levine form $\Lambda$ of $\mathbb{P}(W)$ to $\mathbb{P}\left(W^{\prime} \oplus W^{\perp}\right)$ is the Levine form $\Lambda_{W^{\prime}}$ of $\mathbb{P}\left(W^{\prime}\right)$. Therefore, by (1.4.4), we get

$$
\begin{aligned}
\frac{\alpha}{2} & =\frac{1}{2} \int_{\mathbb{P}\left(W^{\prime} \oplus W^{\perp}\right)} \Lambda_{W^{\prime}} \mu^{p+q+1-N}-\frac{1}{2} \int_{\mathbb{P}^{N}(\mathbb{C})} \Lambda \mu^{q+1} \\
& =\left(\sigma_{p}-\sigma_{p+q-N}-\sigma_{N-1-q}\right)-\left(\sigma_{N}-\sigma_{N-1-q}-\sigma_{q}\right) \\
& =\sigma_{p}+\sigma_{q}-\sigma_{N}-\sigma_{p+q-N} .
\end{aligned}
$$

This completes the proof of (5.1.2).

When there exists a cycle $Y$ in $\mathbb{P}(W)$ such that $X=\overline{f^{*}(Y)}$, we have, for any $\lambda \in \mathbb{C}^{*}$ :

$$
\phi_{\lambda}(X)=X
$$

Therefore $\Phi_{1}=\Phi_{0}, \psi(1)=\psi(0)$, and (5.1.2) becomes an equality.

Conversely, when (5.1.2) is an equality, the convex function $\chi$ satisfies

$$
\lim _{x \rightarrow-\infty} \chi(x)=\chi(0),
$$

and hence is constant on $\mathbb{R}_{-}$. Therefore, $\psi$ is constant on the disk $D=\{z \in$ $\mathbb{C},|z| \leq 1\}$, and, according to (5.1.4),

$$
\operatorname{pr}_{1 *}\left(\delta_{\Phi} \cdot \operatorname{pr}_{2}^{*} \mu^{p+1}\right)=0 \quad \text { on } \stackrel{\circ}{D} .
$$


It follows that

$$
\int_{D \times \mathbb{P}^{N}(\mathbb{C})} \delta_{\Phi} \cdot \operatorname{pr}_{2}^{*} \mu^{p+1}=0
$$

Let $V$ be the regular locus of $|\Phi| \cap\left(\stackrel{\circ}{D} \times \mathbb{P}^{N}(\mathbb{C})\right)$. Since $\Phi$ is effective and $\mu^{p+1}$ positive, (5.1.5) implies that

$$
\operatorname{pr}_{2_{\mid V}}^{*} \mu^{p+1}=0
$$

As the $(1,1)$ form $\mu$ is strictly positive, the restriction of $\mu^{p+1}$ to any $(p+1)$ dimensional complex submanifold of $\mathbb{P}^{N}(\mathbb{C})$ is a strictly positive volume form, and (5.1.6) shows that the map $\operatorname{pr}_{2}: V \rightarrow \mathbb{P}^{N}(\mathbb{C})$ has everywhere rank smaller than $p+1$. It follows that $V$ may be written $\stackrel{\circ}{D} \times V_{0}$, where $V_{0}$ is some (locally closed) submanifold of $\mathbb{P}^{N}(\mathbb{C})$. This implies that the (analytic) cycle $\Phi \cap\left(\stackrel{\circ}{D} \times \mathbb{P}^{N}(\mathbb{C})\right)$ in $\stackrel{\circ}{D} \times \mathbb{P}^{N}(\mathbb{C})$ may be written $\stackrel{\circ}{D} \times \Phi_{0}$ for some cycle $\Phi_{0}$ in $\mathbb{P}^{N}(\mathbb{C})$. Therefore, $\Phi_{\lambda}$ does not depend on $\lambda \in \stackrel{\circ}{D}$, and finally:

$$
\Phi_{1}=\Phi_{0}=\overline{f^{*}(X \cdot \mathbb{P}(W))} .
$$

Remarks. (i) Inequality (5.1.2) is strictly stronger than the inequality

$$
\begin{aligned}
\frac{1}{2} \int_{\mathbb{P}^{N}(\mathbb{C})} \delta_{X} g_{\mathbb{P}(W)} \mu^{p+q+1-N} & \geq-\frac{1}{2} \int \delta_{X} H(\Lambda) \mu^{p+q+1-N} \\
& =\operatorname{deg}(X)\left(\sigma_{q}+\sigma_{N-q-1}-\sigma_{N}\right)
\end{aligned}
$$

which follows from the pointwise inequality $\Lambda \geq 0$.

(ii) Clearly, inequality (5.1.7) still holds when $p+q=N-1$ provided $X \in Z_{p}\left(\mathbb{P}^{N}(\mathbb{C})\right)$ is effective and meets $\mathbb{P}(W)$ properly (i.e., if $|X|$ and $\mathbb{P}(W)$ do not meet). This shows that (5.1.2) still holds when $p+q=N-1$. As $\Lambda_{\mid \mathbb{P}\left(W^{\perp}\right)}=0$, we see that, when $p+q=N-1$, (5.1.2) or (5.1.7) become equalities when $X$ is a multiple of $\mathbb{P}\left(W^{\perp}\right)$. Moreover, it immediately follows from the expression (1.2.13) for $\Lambda$ and the strict positivity of $\mu$ that this occurs only in this case.

(iii) The equality

$$
\int_{\mathbb{P}^{N}(\mathbb{C})} \delta_{\mathbb{P}\left(W^{\perp}\right)} g_{\mathbb{P}(W)}=2\left(\sigma_{q}+\sigma_{N-q-1}-\sigma_{N}\right)
$$

holds in fact for any $\mu$-normalized Green form for $\mathbb{P}(W)$. This follows from Stokes's formula, together with the fact that the difference of two $\mu$-normalized Green forms for $\mathbb{P}(W)$ may be written $\partial u+\bar{\partial} v$, where $u$ and $v$ are currents $C^{\infty}$ on $\mathbb{P}^{N}(\mathbb{C})-\mathbb{P}(W)$. If $g$ is any positive Green form for $\mathbb{P}(V)$ such that

$$
d d^{c} g+\delta_{\mathbb{P}(W)}=\mu^{N-q},
$$


this equality applied to

$$
g_{\mathbf{P}(W)}=g-H(g)=g-\left(\int_{\mathbb{P}^{N}(\mathbb{C})} g \mu^{q+1}\right) \mu^{N-q-1}
$$

proves that

$$
\int_{\mathbb{P}^{N}(\mathbb{C})} g \mu^{q+1}=\int_{\mathbb{P}^{N}(\mathbb{C})} \delta_{\mathbb{P}\left(W^{\perp}\right)}\left(g-g_{\mathbb{P}(W)}\right) \geq 2\left(\sigma_{N}-\sigma_{q}-\sigma_{N-q-1}\right),
$$

as was announced in 1.4.3, (iv).

\subsection{Lower bounds for the projective height.}

5.2.1. Let $\bar{E}$ be an hermitian vector bundle over $S$ of rank $N+1$, where $N \geq 1$, and let $h^{\bar{E}}$ (resp. $h_{F}$ ) be the associated projective height (resp. Faltings height) of cycles in $\mathbb{P}(E)$ (cf. 4.1.1 and 3.1.2.3).

The following proposition is a first application of the basic inequality (5.1.2).

Proposition 5.2.1. Let $\bar{F} \subset \bar{E}$ be any subbundle of rank $N$ in $E$, equipped with the induced metric, and let $Z$ be a cycle on $\mathbb{P}(E)$ of dimension $p \in\{1, \ldots, N+$ 1) such that $Z_{K}$ is effective and meets $\mathbb{P}(F)_{K}$ properly in $\mathbb{P}(E)_{K}$. For any representative $Z . \mathbb{P}(F)$ of the intersection class $[Z] .[\mathbb{P}(F)] \in C H_{|Z| \cap \mathbb{P}(F)}^{N+2-p}(\mathbb{P}(E))$, we have:

$$
h^{\bar{F}}(Z . \mathbb{P}(F)) \leq h^{\bar{E}}(Z) \text { if } p \geq 2 \text {; }
$$

$$
h^{\bar{F}}(Z \cdot \mathbb{P}(F)) \leq h^{\bar{E}}(Z)-\operatorname{deg}_{K} Z . \widehat{\operatorname{deg}} \bar{F} \quad \text { if } p=1 .
$$

Equality holds in (5.2.1) (resp. in (5.2.2)) if and only if, for any embedding $\sigma: K \rightarrow \mathbb{C}, Z_{\sigma}$ is the inverse image of a cycle on $\mathbb{P}(F)_{\sigma}$ by the orthogonal projection $\mathbb{P}(E)_{\sigma} \rightarrow \mathbb{P}(F)_{\sigma}$ defined using the hermitian metric on $E_{\sigma}$ (resp. a multiple of the center $\mathbb{P}\left(F_{\sigma}^{\perp}\right)$ of this projection).

Proof. Using (4.1.2), (4.1.2'), and the equality

$$
\operatorname{deg}_{K} Z=\operatorname{deg}_{K}(Z . \mathbb{P}(F))
$$

if $p \geq 2$, we see that (5.2.1) and (5.2.2) are equivalent to

$$
h_{F}(Z . \mathbb{P}(F)) \leq h_{F}(Z)+\operatorname{deg}_{K} Z \cdot\left[\widehat{\operatorname{deg}}(\bar{E})-\widehat{\operatorname{deg}}(\bar{F})+[K: \mathbb{Q}]\left(\sigma_{p-2}-\sigma_{p-1}\right)\right] .
$$

As $\mathbb{P}(E)$ is a projective bundle over $S$, the map

$$
\begin{aligned}
C H^{1}(S) & \oplus \mathbb{Z} \rightarrow C H^{1}(\mathbb{P}(E)) \\
x \oplus k & \mapsto \pi^{*}(x)+c_{1}\left(\mathscr{\sigma}_{E}(k)\right)
\end{aligned}
$$

is an isomorphism. Therefore, if $h$ denotes the order of $C H^{1}(S)$ (which is nothing else than the ideal class group of $K)$, there exists $s \in H^{0}\left(\mathbb{P}(E) ; \mathscr{O}_{E}(h)\right)$ 
such that $\operatorname{div} s=h \mathbb{P}(F)$. Let $\mu=c_{1}\left(\overline{\mathscr{O}_{E}(1)}\right)$. Formula (3.2.2) for the height of a divisor shows that

$$
h_{F}(Z . \mathbb{P}(F))=h_{F}(Z)+h^{-1} \int_{\mathbb{P}(E)(\mathbb{C})} \log \|s\| \cdot \delta_{Z} \cdot \mu^{p-1}
$$

and

$$
h_{F}(\mathbb{P}(F))=h_{F}(\mathbb{P}(E))+h^{-1} \int_{\mathbf{P}(E)(\mathbb{C})} \log \|s\| \mu^{N} .
$$

On the other hand, for any embedding $\sigma: K \rightarrow \mathbb{C}$, the function

$$
h^{-1} \log \|s\|^{-2}-\int_{\mathbb{P}(E)_{\sigma}(\mathbb{C})} h^{-1} \log \|s\|^{-2} \mu^{N}
$$

on $\mathbb{P}(E)_{\sigma}(\mathbb{C})$ is the $\mu$-normalized Green form for $\mathbb{P}(F)_{\sigma}$. Therefore we get from Proposition 5.1.1 applied to $X=Z_{\sigma}$ :

$$
\begin{aligned}
& h^{-1} \int_{\mathbb{P}(E)_{\sigma}(\mathbb{C})} \log \|s\|^{-1} \delta_{Z} \mu^{p-1}-\operatorname{deg}_{K} Z \cdot h^{-1} \int_{\mathbb{P}(E)_{\sigma}(\mathbb{C})} \log \|s\|^{-1} \mu^{N} \\
& \quad \geq \operatorname{deg}_{K} Z \cdot\left(\sigma_{p-1}+\sigma_{N-1}-\sigma_{N}-\sigma_{p-2}\right)
\end{aligned}
$$

indeed

$$
\int_{\mathbb{P}(E)_{\sigma}(\mathbb{C})} \delta_{Z} \mu^{p}=\operatorname{deg}\left(Z_{\sigma}\right)=\operatorname{deg}_{K}(Z) .
$$

Inequality (5.2.3) follows from (5.2.4)-(5.2.6), together with the following consequence of formula (4.1.4) for the Faltings height of a projective space:

$$
h_{F}(\mathbb{P}(F))-h_{F}(\mathbb{P}(E))=\widehat{\operatorname{deg}} \bar{E}-\widehat{\operatorname{deg}} \bar{F}+[K: \mathbb{Q}]\left(\sigma_{N-1}-\sigma_{N}\right) .
$$

Proposition 5.2.2. Let $F_{1}, \ldots, F_{N+1}$ be subbundles of rank $N$ in $E$ such that $\cap_{i=1}^{N+1} F_{i, K}=0$, and, for any nonempty subset $I \subset\{1, \ldots, N+1\}$, let $\bar{F}_{I} \subset \bar{E}$ be the subbundle $\bigcap_{i \in I} F_{i}$ of $E$ (i.e., the bundle attached to the intersection of the $\mathscr{O}_{K}$-submodules corresponding to $\left.F_{i}, i \in I\right)$, equipped with the induced metric. Then, for any effective cycle $Z \in Z_{p}(\mathbb{P}(E))$,

$$
\begin{gathered}
h^{\bar{E}}(Z) \geq 0 \text { if } p=0 \\
h^{\bar{E}}(Z) \geq \operatorname{deg}_{K} Z . \inf _{\substack{I \subset\{1, \ldots, N+1\} \\
\| I I=p}} \widehat{\operatorname{deg}} \bar{F}_{I} \text { if } p \in\{1, \ldots, N+1\} .
\end{gathered}
$$

Proof. First observe that Proposition 4.1.2, (i) and formula (3.1.3) imply that

(5.29) for any nonzero effective cycle $Z$ supported by closed fibers in $\mathbb{P}(E)$,

$$
h^{\bar{E}}(Z)=h_{F}(Z)>0 \text {. }
$$


This proves (5.2.7) and shows that, to establish (5.2.8), we may assume that $Z$ is irreducible and flat over $S$. Moreover, as $h^{\bar{E}}(\mathbb{P}(E))=0,(5.2 .8)$ clearly holds when $p=N+1$, and we may assume that $p \in\{1, \ldots, N\}$.

Let $Z \in Z_{p}(\mathbb{P}(E))$ be such a cycle. As

$$
Z_{K} \neq \varnothing \text { and } \bigcap_{i=1}^{N+1} \mathbb{P}\left(F^{i}\right)_{K}=\varnothing
$$

there exists $i_{0} \in\{1, \ldots, N+1\}$ such that $Z_{K}$ is not contained in $\mathbb{P}\left(F^{i}\right)_{K}$. Then $Z$ meets $\mathbb{P}\left(F^{i}\right)$ properly, and we can apply Proposition 5.2.1.

Therefore, if $p=1$, we get

$$
h^{\bar{E}}(Z) \geq h^{\bar{F}_{i_{0}}}\left(Z \cdot \mathbb{P}\left(F_{i_{0}}\right)\right)+\operatorname{deg}_{K}(Z) \cdot \widehat{\operatorname{deg}} \bar{F}_{i_{0}} .
$$

As $Z . \mathbb{P}\left(F^{i_{0}}\right)$ is an effective 0 -cycle, $h^{\bar{F}_{i_{0}}}\left(Z . \mathbb{P}\left(F_{i_{0}}\right)\right)$ is nonnegative, and (5.2.8) follows when $p=1$.

If $p \geq 2$, we obtain

$$
h^{\bar{E}}(Z) \geq h^{\bar{F}_{i_{0}}}\left(Z . \mathbb{P}\left(F_{i_{0}}\right)\right) .
$$

Using (5.2.10), inequality (5.2.8) follows by induction on $p$. Indeed, $Z \cdot \mathbb{P}\left(F_{i_{0}}\right)$ is an effective cycle of dimension $p-1$ in $\mathbb{P}\left(F_{i_{0}}\right)$, and (5.2.8) applied to $p-1$, $\bar{F}_{i_{\delta}}, Z . \mathbb{P}\left(F_{i_{0}}\right),\left(F_{i_{0}} \cap F_{i}\right)_{\substack{0 \leq i \leq N \\ i \neq i_{0}}}$ in place of $p, \bar{E}, Z,\left(F_{i}\right)_{0 \leq i \leq N}$ reads:

$$
h^{\overline{F_{i_{0}}}}\left(Z . \mathbb{P}\left(F_{i_{0}}\right)\right) \geq \operatorname{deg}_{K}\left(Z . \mathbb{P}\left(F_{i_{0}}\right)\right) \underset{\substack{I^{\prime} \subset\{1, \ldots, N+1\} \\ m I^{\prime}=p-1 \\ I^{\prime} \nsucceq i_{0}}}{\inf } \widehat{\operatorname{deg}} \overline{F_{I^{\prime} \cup\left\{i_{0}\right\}}}
$$

since

$$
\operatorname{deg}_{K}\left(Z \cdot \mathbb{P}\left(F_{i_{0}}\right)\right)=\operatorname{deg}_{K}(Z),
$$

(5.2.10) and (5.2.11) imply (5.2.8).

5.2.2. Let us assume in this subsection that $\bar{E}$ is the trivial hermitian vector bundle $\overline{\mathscr{O}}_{S}^{\oplus(N+1)}$. Then on $\mathbb{P}(E)=\mathbb{P}_{S}^{N}$ we have canonical homogeneous coordinates $X_{0}, \ldots, X_{N}$ in $H^{0}\left(\mathbb{P}_{S}^{N} ; \mathscr{O}(1)\right)$. For any subset $I \subseteq\{0, \ldots, N\}$, we denote by $\mathbb{P}_{I}$ the linear subspace of codimension $\# I$ in $\mathbb{P}_{S}^{N}$ defined by the equations

$$
X_{i}=0, i \in I \text {. }
$$

Theorem 5.2.3. If $\bar{E}$ is the trivial hermitian vector bundle, then for any effective cycle $Z \in Z_{p}(\mathbb{P}(E))$, we have:

$$
h(Z) \geq 0
$$


Moreover equality holds in (5.2.12) if and only if $Z=0$ when $p=0$, and if and only if $Z$ may be written as

$$
Z=\sum_{\# I=N+1-p} n_{I} \mathbb{P}_{I}
$$

where $n_{I} \in \mathbb{N}$, when $p \in\{1, \ldots, N+1\}$.

Proof. Inequality (5.2.12) follows from Proposition 5.2.2 where one takes as $F_{i}$ the subbundle of $\mathscr{O}_{S}^{\oplus(N+1)}$ defined by the vanishing of the $i$-th component. When equality holds in (5.2.12), $Z$ is necessarily flat over $S$ by (5.2.9), and therefore vanishes when $p=0$. The equality case when $p \geq 1$ follows by induction on $p$ from the proof of Proposition 5.2.2 and the equality case in Proposition 5.2.1. (Observe that the orthogonal projections $\mathbb{P}(E)_{\sigma} \rightarrow \mathbb{P}\left(F_{i}\right)_{\sigma}$ coincide with the projection defined by the morphism $E \rightarrow F_{i},\left(x_{1}, \ldots, x_{N+1}\right) \mapsto$ $\left(x_{1}, \ldots, x_{i-1}, 0, x_{i+1}, \ldots, x_{N+1}\right)$.)

5.2.3. In this subsection, we go back to the case of an arbitrary hermitian vector bundle $\bar{E}$ of rank $N+1$ over $S$, and we give lower bounds for the projective height $h^{\bar{E}}$ in terms of invariants attached to $\bar{E}$ by the geometry of numbers.

For any $p \in\{1, \ldots, N+1\}$, we let:

$$
\begin{aligned}
m_{p} & =\inf \left\{\operatorname{deg}_{K}(Z)^{-1} \cdot h^{\bar{E}}(Z) ; Z \in Z_{p}(\mathbb{P}(E)), Z \text { effective }, Z_{K} \neq 0\right\} \\
& =\inf \left\{\operatorname{deg}_{K}(Z)^{-1} \cdot h^{\bar{E}}(Z) ; Z \in Z_{p-1}\left(\mathbb{P}(E)_{K}\right), Z \text { effective }, Z \neq 0\right\}
\end{aligned}
$$

(the last equality follows from (5.2.9); $m_{p}$ is a real number by 3.2.3, Remark (iii), and (4.1.2)). We also consider the minimal height of a linear subspace:

$$
\begin{aligned}
\ell_{p} & =\inf \left\{h^{\bar{E}}(\mathbb{P}(F)) \mid F \text { subbundle of rank } p \text { in } E\right\} \\
& =\inf \left\{h^{\bar{E}}\left(\mathbb{P}\left(F_{K}\right)\right) \mid F_{K} K \text {-vector subspace of dimension } p \text { in } E_{K}\right\},
\end{aligned}
$$

and the $p$-th minima:

$\lambda_{p}=\inf \left\{\lambda \in \mathbb{R} \mid \exists\left(P_{1}, \ldots, P_{p}\right) \in \mathbb{P}^{N}(E)(K),\left(P_{1}, \ldots, P_{p}\right)\right.$ is projectively

free (i.e., these points are not all contained in any linear subspace of dimension $p-2)$, and $\left.h_{F}\left(P_{1}\right) \leq \lambda, \ldots, h_{F}\left(P_{p}\right) \leq \lambda\right\}$

and

$\lambda_{p}^{\prime}=\inf \left\{\lambda \in \mathbb{R} \mid \exists\left(v_{1}, \ldots, v_{p}\right) \in E^{p}\right.$, the $v_{i}$ 's are $K$-linearly independent , and for any $\sigma: K \hookrightarrow \mathbb{C}$ and any $\left.i=1, \ldots, p, \log \left\|v_{i}\right\|_{\sigma} \leq \lambda\right\}$, where, for any embedding $\sigma: K \hookrightarrow \mathbb{C}$, we denote by \|\|$_{\sigma}$ the norm on $E_{K} \hookrightarrow E \otimes_{\sigma} \mathbb{C}$ defined by the hermitian structure on $\bar{E}$. The sequences $\left(\lambda_{p}\right)_{1 \leq p \leq N+1}$ and $\left(\lambda_{p}^{\prime}\right)_{1 \leq p \leq N+1}$ are increasing. Moreover, there exist sequences 
$\left(Q_{1}, \ldots, Q_{N+1}\right) \in \mathbb{P}(E)(K)^{N+1}$ and $\left(w_{1}, \ldots, w_{N+1}\right) \in E^{N+1}$ such that $\left(Q_{1}, \ldots, Q_{N+1}\right)$ is a projective basis of $\mathbb{P}(E)_{K},\left(w_{1}, \ldots, w_{N+1}\right)$ is a basis of the $K$-vector space $E_{K}$, and for any $p \in\{1, \ldots, N+1\}$

$$
h_{F}\left(Q_{p}\right)=\lambda_{p} \text { and } \sup _{\sigma \in K \hookrightarrow \mathbb{C}} \log \left\|w_{p}\right\|_{\sigma}=\lambda_{p}^{\prime} .
$$

The height $h_{F}\left(\left[w_{p}\right]\right)$ of the point $\left[w_{p}\right] \in \mathbb{P}\left(E_{K}\right)$ is bounded above by

$$
\sum_{\sigma: K \hookrightarrow \mathbb{C}} \log \left\|w_{p}\right\|_{\sigma} \leq[K: \mathbb{Q}] \lambda_{p}^{\prime}
$$

It follows that

$$
\lambda_{p} \leq[K: \mathbb{Q}] \lambda_{p}^{\prime}
$$

Theorem 5.2.4. For any $p \in\{1, \ldots, N+1\}$, the following inequalities hold:

$$
-\sum_{j=p+1}^{N+1} \lambda_{j} \leq m_{p} \leq \ell_{p} \leq \sum_{j=1}^{p} \lambda_{j}+\widehat{\operatorname{deg}} \bar{E}
$$

We may combine inequalities (5.2.15) and (5.2.16), and Minkowski's second theorem in the form established by Bombieri and Vaaler [Bo-Va], namely:

$$
[K: \mathbb{Q}] \sum_{i=1}^{N+1} \lambda_{i}^{\prime} \leq C(N, K)-\widehat{\operatorname{deg}} \bar{E},
$$

where $C(N, K)$ is the following expression involving the numbers $r_{1}$ and $r_{2}$ of real and complex places, the absolute discriminant $\Delta_{K}$ of $K$, and the standard euclidean volume $V_{n}$ of the unit ball in $\mathbb{R}^{n}$ :

$$
C(N, K)=(N+1)\left(r_{1}+r_{2}\right) \log 2+\frac{N+1}{2} \log \left|\Delta_{K}\right|-r_{1} \log V_{N+1}-r_{2} \log V_{2 N+2} .
$$

Then we get:

Corollary 5.2.5. For any $p \in\{1, \ldots, N+1\}$, the following inequalities hold:

$$
\begin{aligned}
\sum_{j=1}^{p} \lambda_{j}+\widehat{\operatorname{deg}} \bar{E}-C(N, K) & \leq[K: \mathbb{Q}] \sum_{j=1}^{p} \lambda_{j}^{\prime}+\widehat{\operatorname{deg}} \bar{E}-C(N, K) \leq m_{p} \\
& \leq \ell_{p} \leq \sum_{j=1}^{p} \lambda_{j}+\widehat{\operatorname{deg}} \bar{E} .
\end{aligned}
$$

In particular, any of the sequences $\left(m_{p}\right),\left(\ell_{p}\right),\left(\lambda_{p}\right)$, and $\left(\lambda_{p}^{\prime}\right)$ determine the other ones up to some error term bounded by an expression depending only on $N$ and $K$.

The proof of Theorem 5.2.4 will rely on the following. 
Lemma 5.2.6. Let $\left(P_{1}, \ldots, P_{p}\right)$ be a projective free family of points in $\mathbb{P}(E)(K)$, and let $V$ be the linear subspace of $\mathbb{P}(E)_{K}$ spanned by $\left(P_{1}, \ldots, P_{p}\right)$. Then:

$$
h^{\bar{E}}(V) \leq \sum_{i=1}^{p} h_{F}\left(P_{i}\right)+\widehat{\operatorname{deg}} \bar{E} .
$$

Proof. Let $\bar{L}_{i} \subset \bar{E}$ be the subbundle of rank 1 of $E$ such that $\mathbb{P}\left(L_{i}\right)(K)=$ $\left\{P_{i}\right\}$, equipped with the induced metric. Consider the rank $p$ subbundle $F=$ $\left(\sum_{i=1}^{p} L_{i}\right)_{K} \cap E$ of $E$; the subscheme $\mathbb{P}(F)$ of $\mathbb{P}(E)$ coincides with the Zariski closure of $V$. According to (4.1.3) and to the definition of $h_{F}$, we have

$$
h^{\bar{E}}(V)=\widehat{\operatorname{deg}}(\bar{E})-\widehat{\operatorname{deg}}(\bar{F})
$$

and

$$
h_{F}\left(P_{i}\right)=-\widehat{\operatorname{deg}}\left(\bar{L}_{i}\right)
$$

Therefore, (5.2.17) is equivalent to

$$
\widehat{\operatorname{deg}}(\bar{F})-\sum_{i=1}^{p} \widehat{\operatorname{deg}}\left(\bar{L}_{i}\right) \geq 0
$$

The injections $L_{i} \hookrightarrow F$ define a map

$$
\varphi: \bigoplus_{i=1}^{p} L_{i} \rightarrow F,
$$

which is an isomorphism over $\operatorname{Spec}(K)$. Thus the map

$$
\Lambda^{p} \varphi: \Lambda^{p}\left(\bigoplus_{i=1}^{p} L_{i}\right) \simeq \bigotimes_{i=1}^{p} L_{i} \rightarrow \operatorname{det} F
$$

is nonzero, and defines a nonzero section of the hermitian line bundle $\operatorname{det} \bar{F} \otimes$ $\bigotimes_{i=1}^{p} \bar{L}_{i}^{\vee}$, which is easily seen to have norms $\leq 1$. This implies that

$$
\widehat{\operatorname{deg}}(\bar{F})-\sum_{i=1}^{p} \widehat{\operatorname{deg}}\left(\bar{L}_{i}\right)=\widehat{\operatorname{deg}}\left(\operatorname{det} \bar{F} \otimes \bigotimes_{i=1}^{p} \bar{L}_{i}^{\vee}\right)
$$

is nonnegative and proves (5.2.18).

Proof of Theorem 5.2.4. The inequality $m_{p} \leq \ell_{p}$ is trivial, and the inequality $\ell_{p} \leq \sum_{j=1}^{p} \lambda_{j}+\widehat{\operatorname{deg}} \bar{E}$ follows from Lemma 5.2.6 applied to the sequence $\left(P_{1}, \ldots, P_{p}\right)=\left(Q_{1}, \ldots, Q_{p}\right)$. To prove the first inequality in (5.2.16), consider the rank 1 vector spaces $L_{p, K} \subset E_{K}$ defining $Q_{p}, 1 \leq p \leq N+1$, and 
apply Proposition 5.2.2 to the subbundles $F_{0}, \ldots, F_{N}$ such that

$$
F_{i, K}=\bigoplus_{\substack{j=1 \\ j \neq i}}^{N+1} L_{j, K} .
$$

Then, for any $p \in\{1, \ldots, N\}$ and any subset $I \subset\{1, \ldots, N+1\}$ such that $\# I=p$, the subscheme $\mathbb{P}\left(F_{I}\right)$ is the Zariski closure of the linear subspace of $\mathbb{P}\left(E_{K}\right)$ spanned by $\left\{Q_{j}\right\}_{j \in\{1, \ldots, N+1\} \backslash I}$. Therefore, formula (4.1.3) and Lemma 5.2.6 show that

$$
\begin{aligned}
\widehat{\operatorname{deg}} \bar{F}_{I} & =-h^{\bar{E}}\left(\mathbb{P}\left(F_{I}\right)\right)+\widehat{\operatorname{deg}} \bar{E} \\
& \geq-\sum_{j \in\{1, \ldots, N+1\} \backslash I} h_{F}\left(Q_{j}\right) \geq-\sum_{j=p+1}^{N+1} \lambda_{j} .
\end{aligned}
$$

Since, according to $(5.2 .8), m_{p} \geq \inf _{\substack{I \subset\{1, \ldots, N+1\} \\ I I=p}} \widehat{\operatorname{deg}} \bar{F}_{I}$, this proves the required inequality.

Remark. One may wonder if there exists a lower bound of $\ell_{p}-m_{p}$ which involves only $N$ and $[K: \mathbb{Q}]$, and if $m_{p}$ may be expressed in terms of the canonical polygon of $\bar{E}$ as defined by Stuhler [Stu] and Grayson [Gr] (observe that $[K: \mathbb{Q}]^{-1} \ell_{p}$ is unchanged by extension of the ground field $K$, and that, up to some trivial normalization, the same is true for the canonical polygon of $\bar{E})$.

5.3. The fundamental class of an intersection. Let $X$ be a projective regular arithmetic variety of pure dimension $d$ and $\mu \in A^{1,1}\left(X_{\mathbb{R}}\right)$ a Kähler form. As in 2.3.2, we denote by $H$ the harmonic projection of currents and, for any cycle $Y \in Z^{p}(X)$, we let $[Y]_{\mu} \in \widehat{C H}^{p}(X)$ be the class of $\left(Y, g_{Y}\right)$, where $g_{Y}$ is a Green form for $Y$ of $\log$ type along $|Y|$, which is $\mu$-normalized, i.e., which satisfies the normalization conditions $d d^{c}\left(g_{Y}\right)+\delta_{Y}=H\left(\delta_{Y}\right)$ and $H\left(g_{Y}\right)=0$. Recall that $[Y]_{\mu}=0$ if $Y$ is the divisor of a rational function on a subvariety contained in a closed fiber of $X$ over $S$. It follows that, if $Y \in Z^{p}(X)$ and $Z \in Z^{q}(X)$ are cycles on $X$ which meet properly on $X_{K}$, the class $[Y . Z]_{\mu} \in$ $\widehat{C H}^{p+q}(X)_{\mathbb{Q}}$ is well defined (provided $p+q \leq d$ ), independently of the choice of a representative $Y . Z$ for the intersection cycle $[Y] .[Z] \in C H_{|Y| \cap|Z|}^{p+q}(X)_{\mathbb{Q}}$.

Proposition 5.3.1. Assume that the product of two harmonic forms (with respect to $\mu$ ) on $X(\mathbb{C})$ is still harmonic. Then the following equality holds in $\widehat{C H}^{p+q}(X)_{\mathbb{Q}}$ :

$$
[Y . Z]_{\mu}=[Y]_{\mu}[Z]_{\mu}-a\left(H\left(g_{Y} \delta_{Z}\right)\right) .
$$

Proof. Let $g_{Z}$ be a $\mu$-normalized Green form for $Z$ of $\log$ type along $|Z|$. Then $[Y]_{\mu}[Z]_{\mu}$ is the class of 


$$
\left(Y . Z, g_{Y} \delta_{Z}+H\left(\delta_{Y}\right) g_{Z}\right) .
$$

The form

$$
d d^{c}\left(g_{Y} \delta_{Z}+H\left(\delta_{Y}\right) g_{Z}\right)+\delta_{Y . Z}=\omega\left([Y]_{\mu}[Z]_{\mu}\right)=H\left(\delta_{Y}\right) H\left(\delta_{Z}\right)
$$

is harmonic by our hypothesis, so it coincides with $H\left(\delta_{Y . Z}\right)$. It follows that

$$
g_{Y . Z}=g_{Y} \delta_{Z}+H\left(\delta_{Y}\right) g_{Z}-H\left(g_{Y} \delta_{Z}+H\left(\delta_{Y}\right) g_{Z}\right)
$$

is a $\mu$-normalized Green current for $Y . Z$. Since, by our hypothesis again,

$$
H\left(H\left(\delta_{Y}\right) g_{Z}\right)=H\left(\delta_{Y}\right) H\left(g_{Z}\right)=0
$$

we get

$$
[Y]_{\mu}[Z]_{\mu}=\left[\left(Y . Z, g_{Y . Z}+H\left(g_{Y} \delta_{Z}\right)\right)\right]=[Y . Z]_{\mu}+a\left(H\left(g_{Y} \delta_{Z}\right)\right) .
$$

\subsection{Arithmetic Bézout theorem.}

5.4.1. Computations in $C H^{*}(\overline{\mathbb{P}(E)})$. Let $\bar{E}$ be an hermitian vector bundle of rank $N+1$ over $S, \overline{\mathbb{P}(E)}=(\mathbb{P}(E), \mu)$ its projective bundle equipped with the Kähler form $\mu=c_{1}\left(\overline{\mathscr{O}_{E}(1)}\right)$, and $\pi: \mathbb{P}(E) \rightarrow S$ the projection. Denote by $C H^{*}(\overline{\mathbb{P}(E)}) \subset \widehat{C H}^{*}(\mathbb{P}(E))$ the subring consisting of those elements $x$ such that the form $\omega(x)$ is harmonic ( see [G-S2], 5.1). Given $x$ in $C H^{p}(\overline{\mathbb{P}(E)})$ we define

$$
h_{F}(x)=\widehat{\operatorname{deg}} \pi_{*}\left(\widehat{c}_{1}\left(\overline{\mathscr{O}_{E}(1)}\right)^{N+1-p} x\right) \in \mathbb{R}
$$

and, if $p \leq N$,

$$
\operatorname{deg}_{K}(x)=\operatorname{deg}_{K} \pi_{*}\left(\widehat{c}_{1}\left(\overline{\mathscr{O}_{E}(1)}\right)^{N-p} x\right) \in \mathbb{Z} .
$$

When $x=[Y]_{\mu}$ one recovers $h_{F}(Y)$ and $\operatorname{deg}_{K}(Y)$ defined as in (3.1.5) and 4.1.1; this follows from Lemma 2.3.2.

Proposition 5.4.1. Let $x \in C H^{p}(\overline{\mathbb{P}(E)})$ and $y \in C H^{q}(\overline{\mathbb{P}(E)})$. Then, if $\max (p, q, p+q) \leq N$,

$$
\operatorname{deg}_{K}(x y)=\operatorname{deg}_{K}(x) \operatorname{deg}_{K}(y)
$$

and, if $\max (p, q) \leq N$ and $p+q \leq N+1$,

$$
\begin{aligned}
h_{F}(x y)= & h_{F}(x) \operatorname{deg}_{K}(y)+\operatorname{deg}_{K}(x) h_{F}(y) \\
& +\operatorname{deg}_{K}(x) \operatorname{deg}_{K}(y)\left(\widehat{\operatorname{deg}}(\bar{E})-[K: \mathbb{Q}] \sigma_{N}\right) .
\end{aligned}
$$

The proof of Proposition 5.4.1 is based on the following description of the group $C H^{*}(\overline{\mathbb{P}(E)})$ : 
Lemma 5.4.2. The map

$$
\widehat{C H}^{*}(S)^{N+1} \rightarrow C H^{*}(\overline{\mathbb{P}(E)})
$$

sending $\left(\alpha_{0}, \ldots, \alpha_{N}\right)$ to $\sum_{i=0}^{N} \pi^{*}\left(\alpha_{i}\right) \widehat{c}_{1}\left(\overline{\mathscr{G}_{E}(1)}\right)^{i}$ is a group isomorphism.

Proof of Lemma 5.4.2. As in [G-S3], Proposition 3.3.2, this result follows from the Five Lemma applied to the exact sequence describing $C H^{*}(\overline{\mathbb{P}(E)})$.

Proof of Proposition 5.4.1. Let

$$
\widehat{\mu}=\widehat{c}_{1}\left(\overline{\mathscr{O}_{E}(1)}\right) \text {. }
$$

By Lemma 5.4.2, $x \in C H^{p}(\overline{\mathbb{P}(E)})$ can be written as

$$
x=\pi^{*}(\alpha) \widehat{\mu}^{p}+\pi^{*}(\beta) \widehat{\mu}^{p-1},
$$

with $\alpha \in \widehat{C H}^{0}(S) \simeq \mathbb{Z}$ and $\beta \in \widehat{C H}^{1}(S)$, where by convention $\widehat{\mu}^{p-1}=0$ and $\beta=0$ when $p=0$.

Then we have $\operatorname{deg}_{K}(x)=\alpha$ and

$$
\begin{aligned}
h_{F}(x) & =\widehat{\operatorname{deg}} \pi_{*}\left(\widehat{\mu}^{N+1-p} x\right) \\
& =\widehat{\operatorname{deg}}\left(\alpha \pi_{*} \widehat{\mu}^{N+1}+\beta \pi_{*} \widehat{\mu}^{N}\right) \\
& =\alpha \widehat{\operatorname{deg}} \pi_{*}\left(\widehat{\mu}^{N+1}\right)+\widehat{\operatorname{deg}}(\beta) \\
& =\operatorname{deg}_{K}(x) h_{\bar{Q}(1)}(\mathbb{P}(E))+\widehat{\operatorname{deg}} \beta \\
& =\operatorname{deg}_{K}(x)\left([K: \mathbb{Q}] \sigma_{N}-\widehat{\operatorname{deg}}(\bar{E})\right)+\widehat{\operatorname{deg}}(\beta)
\end{aligned}
$$

by (4.1.4).

Similarly we may write

$$
y=\operatorname{deg}_{K}(y) \widehat{\mu}^{q}+\pi^{*}(\gamma) \widehat{\mu}^{q-1},
$$

with $\gamma=0$ and $\widehat{\mu}^{q-1}=0$ if $q=0$, and we get:

$$
x y=\operatorname{deg}_{K}(x) \operatorname{deg}_{K}(y) \widehat{\mu}^{p+q}+\left(\pi^{*}(\beta) \operatorname{deg}_{K}(y)+\operatorname{deg}_{K}(x) \pi^{*}(\gamma)\right) \widehat{\mu}^{p+q-1} .
$$

It follows that $\operatorname{deg}_{K}(x y)=\operatorname{deg}_{K}(x) \operatorname{deg}_{K}(y)$ if $p+q \leq N$ and, by (5.4.1) and (4.1.4),

$$
\begin{aligned}
h_{F}(x y)= & \operatorname{deg}_{K}(x) \operatorname{deg}_{K}(y) \widehat{\operatorname{deg}} \pi_{*} \widehat{\mu}^{N+1}+\widehat{\operatorname{deg}}(\beta) \operatorname{deg}_{K}(y)+\operatorname{deg}_{K}(x) \widehat{\operatorname{deg}} \gamma \\
= & h_{F}(x) \operatorname{deg}_{K}(y)+\operatorname{deg}_{K}(x) h_{F}(y) \\
& +\operatorname{deg}_{K}(x) \operatorname{deg}_{K}(y)\left(\widehat{\operatorname{deg}}(\bar{E})-[K: \mathbb{Q}] \sigma_{N}\right) . \quad \square
\end{aligned}
$$

By applying Propositions 5.3.1 and 5.4.1 to $x=[X]_{\mu}$ and $y=[Y]_{\mu}$, and comparing $h_{F}$ and $h$ by Proposition 4.1.2, (i), we get, for any $\mu$-normalized Green current $g_{Y}$ for $Y$, of log type along $|Y|$ : 
Proposition 5.4.3. Let $X \in Z_{p}(\mathbb{P}(E))$ and $Y \in Z_{q}(\mathbb{P}(E))$ be two cycles on $\mathbb{P}(E)$. Assume that $p \geq 1, q \geq 1, p+q \geq N+1$, and that $X$ and $Y$ meet properly on $\mathbb{P}(E)_{K}$. Then, when $p+q>N+1$, we have:

$$
\begin{aligned}
h(X . Y)= & h(X) \operatorname{deg}_{K}(Y)+\operatorname{deg}_{K}(X) h(Y)-\frac{1}{2} \int_{\mathbb{P}(E)(\mathbb{C})} \delta_{X} g_{Y} \mu^{p+q-N-1} \\
& +[K: \mathbb{Q}] \operatorname{deg}_{K}(X) \operatorname{deg}_{K}(Y)\left(\sigma_{p-1}+\sigma_{q-1}-\sigma_{N}-\sigma_{p+q-N-2}\right) ;
\end{aligned}
$$

when $p+q=N+1$, we have:

$$
\begin{aligned}
h(X . Y)= & h(X) \operatorname{deg}_{K}(Y)+\operatorname{deg}_{K}(X) h(Y)-\frac{1}{2} \int_{\mathbb{P}(E)(\mathbb{C})} \delta_{X} g_{Y} \\
& +\operatorname{deg}_{K}(X) \operatorname{deg}_{K}(Y)\left([K: \mathbb{Q}]\left(\sigma_{p-1}+\sigma_{q-1}-\sigma_{N}\right)-\widehat{\operatorname{deg}} \bar{E}\right) .
\end{aligned}
$$

5.4.2. Arithmetic Bézout theorem. Our earlier version of an arithmetic Bézout theorem, Theorem 4.2.3, is improved and extended by the following:

Theorem 5.4.4. Let $\bar{E}$ be an hermitian vector bundle of rank $N+1$ on $S$, and $\mathbb{P}(E)$ the associated projective bundle, and let $X \in Z_{p}(\mathbb{P}(E))$ and $Y \in Z_{q}(\mathbb{P}(E))$ be effective cycles on $\mathbb{P}(E)$. Assume that $p \geq 1, q \geq 1, p+q \geq N+1$, and that $X$ and $Y$ meet properly on $\mathbb{P}(E)_{K}$, and let $X . Y$ be a representative of the intersection class $[X] .[Y] \in C H_{|X| \cap|Y|}^{2 N+2-p-q}(\mathbb{P}(E))$.

(i) If furthermore $Y=\mathbb{P}(F)$ is a linear subspace of $\mathbb{P}(E)$, then

$$
h(X . Y) \leq h(X)+\operatorname{deg}_{K}(X) h(Y) \text { if } p+q>N+1
$$

and

$$
h(X . Y) \leq h(X)+\operatorname{deg}_{K}(X)[h(Y)-\widehat{\operatorname{deg}} \bar{E}] \text { if } p+q=N+1 .
$$

Equality holds in (5.4.4) ( resp. (5.4.5)) if and only if for any imbedding $\sigma$ : $K \rightarrow \mathbb{C}, X_{\sigma}$ is the inverse image of a cycle on $Y_{\sigma}$ by the orthogonal projection $\mathbb{P}(E)_{\sigma} \rightarrow \mathbb{P}\left(F_{\sigma}\right)$ (resp. a multiple of $\mathbb{P}\left(F_{\sigma}^{\perp}\right)$ ).

(ii) In general, when $p+q>N+1$, we have:

$$
\begin{aligned}
h(X . Y) \leq & h(X) \operatorname{deg}_{K}(Y)+\operatorname{deg}_{K}(X) h(Y) \\
& +[K: \mathbb{Q}] \operatorname{deg}_{K}(X) \operatorname{deg}_{K}(Y)\left(N+1-\frac{p+q}{2}\right) \log 2
\end{aligned}
$$

when $p+q=N+1$, we have

$$
\begin{aligned}
h(X . Y) \leq & h(X) \operatorname{deg}_{K}(Y)+\operatorname{deg}_{K}(X) h(Y) \\
& +\operatorname{deg}_{K} X \cdot \operatorname{deg}_{K} Y\left([K: \mathbb{Q}] \cdot \frac{N+1}{2} \log 2-\widehat{\operatorname{deg}} \bar{E}\right) .
\end{aligned}
$$

Observe that assertion (i) extends Proposition 5.2.1.

Proof. Statement (i) follows from Propositions 5.4.3 and 5.1.1 and from 5.1, Remark (ii). 
To prove (ii), we go back to the first part of the proof of Theorem 4.2.3. Suppose $p+q>N+1$. Applying (5.4.4) to $D$ and the join cycle $X \# Y$ in $\mathbb{P}(E \oplus E)$, we get

$$
h^{\bar{E} \oplus \bar{E}}((X \# Y) . D) \leq h^{\bar{E} \oplus \bar{E}}(X \# Y)+\operatorname{deg}_{K}(X \# Y) h^{\bar{E} \oplus \bar{E}}(D) .
$$

Using (4.1.2), (4.2.11), (4.2.12), and the classical Bézout theorem

we get

$$
\operatorname{deg}_{K}((X \# Y) . D)=\operatorname{deg}_{K}(X . Y)=\operatorname{deg}_{K} X \cdot \operatorname{deg}_{K} Y,
$$

$$
\begin{aligned}
& h^{\bar{E} \oplus \bar{E}}((X \# Y) . D)=h^{\bar{E}}(X . Y) \\
& \quad+\left(\widehat{\operatorname{deg}}(\bar{E} \oplus \bar{E})-\widehat{\operatorname{deg}} \bar{E}+[K: \mathbb{Q}] \frac{p+q-N-1}{2} \log 2\right) \operatorname{deg}_{K}(X) \cdot \operatorname{deg}_{K}(Y) .
\end{aligned}
$$

Since the scalar product induced on $E$ by its diagonal imbedding in $\bar{E} \oplus \bar{E}$ is twice the original scalar product, we have, by Proposition 4.1.2, (ii),

$$
h^{\bar{E} \oplus \bar{E}}(D)=\widehat{\operatorname{deg}}(\bar{E} \oplus \bar{E})-\widehat{\operatorname{deg}}(\bar{E})+[K: \mathbb{Q}] \frac{N+1}{2} \log 2 .
$$

Inequality (5.4.6) follows from (5.4.8), (5.4.9), (5.4.10), and Proposition 4.2.2. Inequality (5.4.7) is obtained by a similar proof, where (5.4.8) is replaced by

$$
h^{\bar{E} \oplus \bar{E}}((X \# Y) . D) \leq h^{\bar{E} \oplus \bar{E}}(X \# Y)+\operatorname{deg}_{K}(X \# Y)\left(h^{\bar{E} \oplus \bar{E}}(D)-\widehat{\operatorname{deg}}(\bar{E} \oplus \bar{E})\right)
$$

and (5.4.9) by

$$
h^{\bar{E} \oplus \bar{E}}((X \# Y) . D)=h^{\bar{E}}(X . Y) .
$$

5.4.3. Remarks. (i) Theorem 5.4.4 implies Theorem 4.2 .3 since

$$
\left(N+1-\frac{p+q}{2}\right) \log 2 \leq a(N, p, q) .
$$

Indeed, $p+q-N-2 \leq p+q-1$, therefore $\sigma_{p+q-N-2} \leq \sigma_{p+q-1}$.

(ii) Like Theorem 4.2.3, Theorem 5.4.4 may be applied to bound the height of the intersection of two cycles in $\mathbb{P}(E)_{K}$. Namely, the same proof as for Corollary 4.2.4 shows that for any two integers $p$ and $q$ such that $0 \leq p \leq N$, $0 \leq q \leq N$, and $p+q \geq N-1$, and for any two effective cycles $X \in Z_{p}\left(\mathbb{P}(E)_{K}\right)$ and $Y \in Z_{q}(\mathbb{P}(E))_{K}$ which meet properly, the following inequality holds:

$$
\begin{aligned}
h(X . Y) \leq & h(X) \operatorname{deg}_{K}(Y)+\operatorname{deg}_{K}(X) h(Y) \\
& +[K: \mathbb{Q}] \operatorname{deg}_{K}(X) \operatorname{deg}_{K}(Y) \frac{2 N-p-q}{2} \log 2,
\end{aligned}
$$

if $p+q \geq N$ and (5.4.7) holds when $p+q=N-1$.

(iii) Let $F$ and $G$ be two subbundles of $E$ such that $E=F+G$. Then $X=$ $\mathbb{P}(F)$ and $Y=\mathbb{P}(G)$ are two integral subschemes of $\mathbb{P}(E)$, whose intersection 
(as schemes and as cycles) is $\mathbb{P}(F \cap G)$ (observe that $F \cap G$ is a subbundle of $E$ and that there exist subbundles $F^{\prime}$ and $G^{\prime}$ of $E$ such that $E=F^{\prime} \oplus G^{\prime} \oplus(F \cap G)$, $F=F^{\prime} \oplus(F \cap G)$, and $\left.G=G^{\prime} \oplus(F \cap G)\right)$. Applied to these two cycles, Theorem 5.4.4 together with Proposition 4.1.2, (ii) give the inequality

$$
\widehat{\operatorname{deg}}(\bar{F})+\widehat{\operatorname{deg}}(\bar{G}) \leq \widehat{\operatorname{deg}}(\overline{F \cap G})+\widehat{\operatorname{deg}}(\overline{F+G}),
$$

where the $\mathscr{O}_{K}$-modules $F, G, F \cap G$ are equipped with the metric induced from $\bar{E}$. This inequality is due to Stuhler ([St], Proposition 2) and Grayson ([Gr], Theorem 1.12; see also [Sch], Lemma 2 and Proof of Theorem 2, and [S-V]) and may also be proved as follows: consider the isomorphism of line bundles over $S$

$$
I: \operatorname{det} F \otimes \operatorname{det} G \simeq \operatorname{det}(F \oplus G) \rightarrow \operatorname{det} E \otimes \operatorname{det}(F \cap G)
$$

defined, up to a sign, by the short exact sequence of $\mathscr{O}_{K}$-modules

$$
0 \rightarrow F \cap G \stackrel{i}{\rightarrow} F \oplus G \rightarrow E \rightarrow 0,
$$

where $i(x)=(x,-x)$ and $p(x, y)=x+y$; using formulae (2.1.14) and (2.1.15), we get

$$
\widehat{\operatorname{deg}}(\overline{F \cap G})+\widehat{\operatorname{deg}}(\bar{E})-\widehat{\operatorname{deg}}(\bar{F})-\widehat{\operatorname{deg}}(\bar{G})=-\sum_{\sigma: K \rightarrow \mathbb{C}} \log \|I\|_{\sigma} ;
$$

this is nonnegative, since the value of each archimedean norm $\|I\|_{\sigma}$ of $I$ is at most 1 , as is easily seen using exterior hermitian algebra.

Let us return to the notation of 5.1, and consider two effective cycles $X \in$ $Z_{p}\left(\mathbb{P}^{N}(\mathbb{C})\right)$ and $Y \in Z_{q}\left(\mathbb{P}^{N}(\mathbb{C})\right)$ which meet properly (we assume $0 \leq p \leq N$, $0 \leq q \leq N$, and $p+q \geq N-1)$, and a $\mu$-normalized Green form $g_{Y}$ for $Y$, of log type along $|Y|$. The preceding discussion together with formulae (5.4.2) and (5.4.3) shows that the real number

$$
C(X, Y)=\frac{1}{2} \int_{\mathbb{P}^{N}(\mathbb{C})} \delta_{X} g_{Y} \mu^{p+q-N+1}-\operatorname{deg}(X) \operatorname{deg}(Y)\left(\sigma_{p}+\sigma_{q}-\sigma_{N}-\sigma_{p+q-N}\right)
$$

may be interpreted as some (logarithmic) measure of the "angle" between $X$ and $Y:$ it is independent of the choice of $g_{Y}$ and symmetric in $(X, Y)$ (this follows from Proposition 1.3.1, (ii) and Theorem 1.3.2, (i)), and it reduces to $-\log \|I\|$ in the linear case.

(iv) Observe that, according to the equality case in Theorem 5.4.4, the inequalities (5.4.6) and (5.4.7) are strict except in the trivial case $p=q=N+1$. We conjecture that, under the hypotheses of Theorem 5.4.4, the inequalities

and, if $p+q=N+1$,

$$
h(X . Y) \leq h(X) \operatorname{deg}_{K}(Y)+\operatorname{deg}_{K}(X) h(Y) \text { if } p+q>N+1
$$

$$
h(X . Y) \leq h(X) \operatorname{deg}_{K}(Y)+\operatorname{deg}_{K}(X) h(Y)-\operatorname{deg}_{K}(X) \cdot \operatorname{deg}_{K}(Y) \widehat{\operatorname{deg}} \bar{E}
$$

always hold. This would follow from the nonnegativity of the real number $C(X, Y)$ for any two effective cycles in $\mathbb{P}^{N}(\mathbb{C})$ which meet properly. 
5.5. Arithmetic Bézout theorem for improper intersections. In this section $\mathbb{P}_{S}^{N}$ denotes the standard projective space $\mathbb{P}\left(\mathscr{\sigma}_{S}^{\oplus(N+1)}\right)$ over $S$, and $h$ the projective height on cycles in $\mathbb{P}_{S}^{N}$ defined by the trivial hermitian vector bundle $\bar{\sigma}_{S}^{\oplus(N+1)}$.

Let $X \in Z_{p}\left(\mathbb{P}_{S}^{N}\right)$ and $Y \in Z_{q}\left(\mathbb{P}_{S}^{N}\right)$ be two effective cycles, with $p+q \geq N+1$, and let

$$
|X| \cap|Y|=\cup_{\alpha} W_{\alpha}
$$

be the decomposition into irreducible components of the (set theoretic) intersection of their support. For any $\alpha$, we have

$$
\operatorname{dim} W_{\alpha} \geq p+q-N-1 \text {, }
$$

and we shall say that $\alpha$ is "good" when equality occurs in (5.5.1) and that $\alpha$ is "bad" otherwise. In other words, the $W_{\alpha}$ 's, $\alpha$ good, are the proper components of the intersection of $X$ and $Y$. For any of them, there is a well-defined positive intersection multiplicity $m_{\alpha}$ of $X$ and $Y$ along $W_{\alpha}$, given by Serre's Tor-formula, and we let

$$
(X . Y)_{\mathrm{pr}}=\sum_{\alpha \operatorname{good}} m_{\alpha} W_{\alpha} \quad\left(\in Z_{p+q-N-1}\left(\mathbb{P}^{N}\right)\right) .
$$

This cycle coincides with the image of the product class $[X] .[Y] \in C H_{|X| \cap|Y|}^{*}\left(\mathbb{P}^{N}\right)$ by the canonical map

$$
C H_{|X| \cap|Y|}^{2 N+2-p-q}\left(\mathbb{P}^{N}\right) \simeq C H_{\alpha \text { bad }}^{2 N+2-p-q}\left(\mathbb{P}^{N}\right) \oplus \bigoplus_{\alpha \operatorname{good}} \mathbb{Z} W_{\alpha} \rightarrow \bigoplus_{\alpha \operatorname{good}} \mathbb{Z} W_{\alpha} .
$$

The $W_{\alpha}$ 's, $\alpha$ bad, are the components of $|X| \cap|Y|$, where $X$ and $Y$ meet "with excess", and we let

$$
\begin{gathered}
(X . Y)_{\mathrm{ex}}=\sum_{\alpha \mathrm{bad}} W_{\alpha} \quad\left(\in \bigoplus_{k>p+q-N-1} Z_{k}\left(\mathbb{P}^{N}\right)\right), \\
h\left((X . Y)_{\mathrm{ex}}\right)=\sum_{\alpha \text { bad }} h\left(W_{\alpha}\right),
\end{gathered}
$$

and

$$
\operatorname{deg}_{K}\left((X . Y)_{\text {ex }}\right)=\sum_{\alpha \text { bad }} \operatorname{deg}_{K}\left(W_{\alpha}\right)
$$

If $X, Y$, and $Z$ are any three effective cycles on $\mathbb{P}^{N}$ of respective dimensions $p, q$ and $r$, the associativity of the intersection product for Chow groups with supports implies that

$$
\left((X . Y)_{\mathrm{pr}} \cdot Z\right)_{\mathrm{pr}}=\left(X .(Y . Z)_{\mathrm{pr}}\right)_{\mathrm{pr}} \quad\left(\in Z_{p+q+r-2 N-2}\left(\mathbb{P}^{N}\right)\right) .
$$

Moreover, if $Y$ and $Z$ meet properly:

$$
(X .(Y . Z))_{\mathrm{ex}} \leq\left((X . Y)_{\mathrm{pr}} \cdot Z\right)_{\mathrm{ex}}+\left((X . Y)_{\mathrm{ex}} \cdot Z\right)_{\mathrm{pr}}+\left((X . Y)_{\mathrm{ex}} \cdot Z\right)_{\mathrm{ex}} .
$$


Indeed the left-hand side is the sum of the irreducible components of $|X| \cap|Y| \cap$ $|Z|$ of dimension $>p+q+r-2 N-2$, and any of them appears in one of the cycles on the right-hand side, which are effective. Finally, if $q=r$, we have

$$
(X .(Y+Z))_{\mathrm{pr}}=(X . Y)_{\mathrm{pr}}+(X . Z)_{\mathrm{pr}}
$$

and

$$
(X .(Y+Z))_{\mathrm{ex}} \leq(X . Y)_{\mathrm{ex}}+(X . Z)_{\mathrm{ex}} .
$$

Theorem 5.5.1. (i) Let $F_{1}, \ldots, F_{r}$ be rank $N$ subbundles of $\mathscr{O}_{S}^{\oplus(N+1)}$ such that the subbundle $F=\bigcap_{i=1}^{r} F_{i}$ has rank $N+1-r$. For any effective cycle $Z \in Z_{p}\left(\mathbb{P}_{S}^{N}\right)$ with $p \geq r$, the following inequality holds:

$$
h\left((Z \cdot \mathbb{P}(F))_{\mathrm{pr}}\right)+h\left((Z \cdot \mathbb{P}(F))_{\mathrm{ex}}\right) \leq h(Z)+\operatorname{deg}_{K}(Z) \cdot \sum_{i=1}^{r} h\left(\mathbb{P}\left(F_{i}\right)\right) .
$$

(ii) For any two effective cycles $X \in Z_{p}\left(\mathbb{P}_{S}^{N}\right)$ and $Y \in Z_{q}\left(\mathbb{P}_{S}^{N}\right)$ with $p \geq 1$, $q \geq 1, p+q \geq N+1$, the following inequality holds:

$$
\begin{aligned}
& h\left((X . Y)_{\mathrm{pr}}\right)+h\left((X . Y)_{\mathrm{ex}}\right) \\
& \leq \quad h(X) \operatorname{deg}_{K}(Y)+\operatorname{deg}_{K}(X) h(Y) \\
& \quad+[K: \mathbb{Q}] \operatorname{deg}_{K}(X) \operatorname{deg}_{K}(Y) \frac{N+1}{2} \log 2 .
\end{aligned}
$$

(iii) Let $X$ and $Y$ be two closed integral subschemes in $\mathbb{P}_{S}^{N}$ of positive dimension and let

$$
|X \cap Y|=\cup_{\alpha} W_{\alpha}
$$

be the decomposition of the support of their intersection into irreducible components. Then

$$
\begin{aligned}
\sum_{\alpha} h\left(W_{\alpha}\right) \leq & h(X) \operatorname{deg}_{K}(Y)+\operatorname{deg}_{K}(X) h(Y) \\
& +[K: \mathbb{Q}] \operatorname{deg}_{K}(X) \operatorname{deg}_{K}(Y) \frac{N+1}{2} \log 2 .
\end{aligned}
$$

(Compare with [P2], Théorème 2.)

During the proof we shall also recover the following geometric inequalities, under the hypotheses of (i), (ii), (iii) respectively:

$$
\operatorname{deg}_{K}(Z . \mathbb{P}(F))_{\mathrm{pr}}+\operatorname{deg}_{K}(Z . \mathbb{P}(F))_{\mathrm{ex}} \leq \operatorname{deg}_{K}(Z) \text {, if } p>r,
$$

$\left(5.5 .7^{\prime}\right) \operatorname{deg}_{K}(X . Y)_{\mathrm{pr}}+\operatorname{deg}_{K}(X . Y)_{\mathrm{ex}} \leq \operatorname{deg}_{K}(X) . \operatorname{deg}_{K}(Y)$, if $p+q>N+1$, 


$$
\sum_{\alpha, \operatorname{dim} W_{\alpha}>0} \operatorname{deg}_{K}\left(W_{\alpha}\right) \leq \operatorname{deg}_{K}(X) \cdot \operatorname{deg}_{K}(Y) .
$$

These inequalities are well known: $\left(5.5 .6^{\prime}\right)$ and $\left(5.5 .7^{\prime}\right)$ follow from Vogel's "main theorem" in [V], Chapter II, or from the "refined Bézout theorem" in [Fu2], 12.3; $\left(5.5 .8^{\prime}\right)$ is due originally to Fulton, Lazarsfeld, and MacPherson ([Fu2], Example 8.4.6). Our proof of Theorem 5.5.1, using the join construction, is very similar to the proof of $\left(5.5 .8^{\prime}\right)$ by these last authors and is in the same spirit as [V].

Proof of Theorem 5.5.1. (i) Inequalities (5.5.6) and (5.5.6 ${ }^{\prime}$ ) will be proved together by induction on $r$. Using the nonnegativity of degrees and heights of effective cycles (Theorem 5.2.3) together with (5.5.4) and (5.5.5), we see that it is enough to prove them when $Z$ is irreducible.

Suppose $r=1$ and $Z$ is irreducible. Then, if $Z \not \subset \mathbb{P}\left(F_{1}\right), Z$ and $\mathbb{P}\left(F_{1}\right)$ meet properly; therefore (5.5.6) follows from (5.4.4) and (5.4.5), and (5.5.6') follows from the equality

$$
\operatorname{deg}_{K}\left(Z \cdot \mathbb{P}\left(F_{1}\right)\right)=\operatorname{deg}_{K}(Z) .
$$

When $Z \subset \mathbb{P}\left(F_{1}\right),(Z \cdot \mathbb{P}(F))_{\mathrm{pr}}=0,(Z \cdot \mathbb{P}(F))_{\mathrm{ex}}=Z$, and (5.5.6) and (5.5.6') are trivial.

Suppose now that $r \geq 2$ and let $F^{\prime}$ be the subbundle ${ }_{i=1}^{r-1} F_{i}$ of rank $N-r$ in $\mathscr{O}_{S}^{\oplus(N+1)}$. Then $\mathbb{P}\left(F^{\prime}\right)$ and $\mathbb{P}\left(F_{r}\right)$ meet properly and, in $\mathbb{P}_{K}^{N}$, their (scheme theoretic) intersection coincides with $\mathbb{P}(F)$. Therefore

$$
\mathbb{P}\left(F^{\prime}\right) \cdot \mathbb{P}\left(F_{r}\right)=\mathbb{P}(F)+R,
$$

where $R$ is some effective cycle (supported by closed fibers). Then, by using (5.5.2)-(5.5.5), the nonnegativity of degrees and heights of effective cycles, and (5.5.6) and $\left(5.5 .6^{\prime}\right)$ with $(r, F)$ replaced by $\left(1, F_{r}\right)$ or $\left(r-1, F^{\prime}\right)$ we get:

$$
\begin{aligned}
h((X . & \left.\mathbb{P}(F))_{\mathrm{pr}}\right)+h\left((X \cdot \mathbb{P}(F))_{\mathrm{ex}}\right) \\
= & h\left(\left(X \cdot\left(\mathbb{P}\left(F^{\prime}\right) \cdot \mathbb{P}\left(F_{r}\right)\right)\right)_{\mathrm{pr}}\right)+h\left(\left(X \cdot\left(\mathbb{P}\left(F^{\prime}\right) \cdot \mathbb{P}\left(F_{r}\right)\right)\right)_{\mathrm{ex}}\right) \\
\leq & h\left(\left(\left(X \cdot \mathbb{P}\left(F^{\prime}\right)\right)_{\mathrm{pr}} \cdot \mathbb{P}\left(F_{r}\right)\right)_{\mathrm{pr}}\right)+h\left(\left(\left(X \cdot \mathbb{P}\left(F^{\prime}\right)\right)_{\mathrm{pr}} \cdot \mathbb{P}\left(F_{r}\right)\right)_{\mathrm{ex}}\right) \\
& +h\left(\left(\left(X \cdot \mathbb{P}\left(F^{\prime}\right)\right)_{\mathrm{ex}} \cdot \mathbb{P}\left(F_{r}\right)\right)_{\mathrm{pr}}\right)+h\left(\left(\left(X \cdot \mathbb{P}\left(F^{\prime}\right)\right)_{\mathrm{ex}} \cdot \mathbb{P}\left(F_{r}\right)\right)_{\mathrm{ex}}\right) \\
\leq & h\left(\left(X \cdot \mathbb{P}\left(F^{\prime}\right)\right)_{\mathrm{pr}}\right)+\operatorname{deg}_{K}\left(X \cdot \mathbb{P}\left(F^{\prime}\right)\right)_{\mathrm{pr}} \cdot h\left(\mathbb{P}\left(F_{r}\right)\right) \\
& +h\left(\left(X \cdot \mathbb{P}\left(F^{\prime}\right)\right)_{\mathrm{ex}}\right)+\operatorname{deg}_{K}\left(X \cdot \mathbb{P}\left(F^{\prime}\right)\right)_{\mathrm{ex}} \cdot h\left(\mathbb{P}\left(F_{r}\right)\right) \\
\leq & h\left(\left(X \cdot \mathbb{P}\left(F^{\prime}\right)\right)_{\mathrm{pr}}\right)+h\left(\left(X \cdot \mathbb{P}\left(F^{\prime}\right)\right)_{\mathrm{ex}}\right)+\operatorname{deg}_{K}(X) \cdot h\left(\mathbb{P}\left(F_{r}\right)\right) \\
\leq & h(X)+\operatorname{deg}_{K}(X) \cdot \sum_{i=1}^{r-1} h\left(\mathbb{P}\left(F_{i}\right)\right)+\operatorname{deg}_{K}(X) \cdot h\left(\mathbb{P}\left(F_{r}\right)\right) .
\end{aligned}
$$


This proves (5.5.6). The proof of $\left(5.5 .6^{\prime}\right)$ is similar, but simpler.

(ii) To prove (5.5.7) and $\left(5.5 .7^{\prime}\right)$, we use the join construction, as in the proof of Theorems 4.2.3 and 5.4.4, (ii). Let $j: \mathbb{P}_{S}^{N} \rightarrow \mathbb{P}_{S}^{2 N+1}, D \subset \mathbb{P}_{S}^{2 N+1}$, and $H_{i} \subset \mathbb{P}_{S}^{2 N+1}$ be as in 4.2.3 and let $X \# Y \in Z_{p+q}\left(\mathbb{P}_{S}^{2 N+1}\right)$ be the join of $X$ and $Y$. From [Fu2], Examples 8.4.5, we know that $j:|X| \cap|Y| \rightarrow|X \# Y| \cap D$ is an isomorphism, and that

$$
j_{*}\left((X . Y)_{\mathrm{pr}}\right)=((X \# Y) . D)_{\mathrm{pr}}
$$

and

$$
j_{*}\left((X . Y)_{\mathrm{ex}}\right)=((X \# Y) \cdot D)_{\mathrm{ex}} \cdot
$$

Moreover, using (3.2.1) and (3.2.4), and the fact that the canonical isomorphism $j^{*}(\mathscr{O}(-1)) \simeq \mathscr{O}(-1)$ divides scalar products by 2 , we obtain that, for any $Z \in Z_{r}\left(\mathbb{P}_{S}^{N}\right)$,

$$
h(Z)=h\left(j_{*}(Z)\right)-[K: \mathbb{Q}] \operatorname{deg}_{K}(Z) \cdot \frac{r}{2} \log 2 \leq h\left(j_{*}(Z)\right) .
$$

We deduce from (5.5.9), (5.5.10), (5.5.11) that

$$
h\left((X . Y)_{\mathrm{pr}}\right)+h\left((X . Y)_{\mathrm{ex}}\right) \leq h\left(((X \# Y) \cdot D)_{\mathrm{pr}}\right)+h\left(((X \# Y) \cdot D)_{\mathrm{ex}}\right) .
$$

Inequality (5.5.6), when applied to $Z=X \# Y, r=N+1, \mathbb{P}\left(F_{1}\right)=H_{0}, \ldots$, $\mathbb{P}\left(F_{N+1}\right)=H_{N}$, gives

$$
\begin{aligned}
& h\left(((X \# Y) \cdot D)_{\mathrm{pr}}\right)+h\left(((X \# Y) \cdot D)_{\mathrm{ex}}\right) \\
& \quad \leq h(X \# Y)+[K: \mathbb{Q}] \operatorname{deg}_{K}(X \# Y) \cdot \frac{N+1}{2} \log 2 ;
\end{aligned}
$$

indeed $h\left(\mathbb{P}\left(H_{i}\right)\right)=\frac{1}{2}[K: \mathbb{Q}] \log 2$. Together with Proposition 4.2.2, inequalities (5.5.12) and (5.5.13) prove (5.5.7). Inequality $\left(5.5 .7^{\prime}\right)$ follows from the same argument, where the height $h$ is replaced by the degree $\operatorname{deg}_{K}$, and (5.5.11) by the equality $\operatorname{deg}_{K}(Z)=\operatorname{deg}_{K}\left(j_{*}(Z)\right)$.

(iii) When $\operatorname{dim} X+\operatorname{dim} Y \geq N+1,(5.5 .8)$ and $\left(5.5 .8^{\prime}\right)$ follow from (5.5.7) and $\left(5.5 .7^{\prime}\right)$. The general case may be proved along the same lines as (5.5.7): first, an argument similar to the one in (i), but simpler, shows by induction on $r$ that if $Y=\mathbb{P}(F)$ with $F$ as in (i), we have

$$
\sum_{\alpha} h\left(W_{\alpha}\right) \leq h(X)+\operatorname{deg}_{K}(X) \cdot \sum_{i=1}^{r} h\left(\mathbb{P}\left(F_{i}\right)\right)
$$

and

$$
\sum_{\substack{\alpha \\ \operatorname{dim} W_{\alpha}>0}} \operatorname{deg}_{K}\left(W_{\alpha}\right) \leq \operatorname{deg}_{K}(X)
$$


then one deduces (5.5.8) and $\left(5.5 .8^{\prime}\right)$ from (5.5.14) and $\left(5.5 .14^{\prime}\right)$ by the join construction. One may also reduce to the case where $\operatorname{dim} X+\operatorname{dim} Y \geq N+$ 1 by considering $X \# \mathbb{P}_{S}^{N}$ and $Y \# \mathbb{P}_{S}^{N}$ in $\mathbb{P}_{S}^{2 N+1}$ (observe that $\operatorname{dim}\left(X \# \mathbb{P}_{S}^{N}\right)+$ $\operatorname{dim}\left(Y \# \mathbb{P}_{S}^{N}\right) \geq 2 \operatorname{dim} \mathbb{P}_{S}^{N}=2 N+2$ and that, scheme theoretically, $(X \cap Y) \# \mathbb{P}_{S}^{N}=$ $\left(X \# \mathbb{P}_{S}^{N}\right) \cap\left(Y \# \mathbb{P}_{S}^{N}\right)$, and use that for $Z \in Z_{*}\left(\mathbb{P}_{S}^{N}\right), h\left(Z \# \mathbb{P}_{S}^{N}\right)=h(Z)$ and $\left.\operatorname{deg}_{K}\left(Z \# \mathbb{P}_{S}^{N}\right)=\operatorname{deg}_{K}(Z)\right)$. In that way we get $\left(5.5 .8^{\prime}\right)$, and (5.5.8) with $\frac{N+1}{2}$ replaced by $N+1$.

5.6. Variants. Theorem 5.4 .4 can be extended to the case of several effective cycles $X_{i} \in Z_{p_{i}}(\mathbb{P}(E)), i=1, \ldots, k, p_{i}=1, \ldots, N+1$. When $\sum_{i=1}^{k}\left(p_{i}-1\right) \geq$ $(k-1) N$ and when the cycles $Z_{i}$ meet properly on the generic fiber (i.e., when any component of $\bigcap_{i=1}^{k}\left|Z_{i}\right|_{K}$ has dimension $\left.\sum_{i=1}^{k}\left(p_{i}-1\right)-(k-1) N\right)$, we get

$$
\begin{aligned}
h\left(X_{1}, \cdots . X_{k}\right) \leq & \sum_{i=1}^{k} h\left(X_{i}\right)\left(\prod_{j \neq i} \operatorname{deg}_{K}\left(X_{j}\right)\right) \\
& +[K: \mathbb{Q}] \prod_{i=1}^{k} \operatorname{deg}_{K}\left(X_{i}\right) \cdot \frac{1}{2}\left(k(N+1)-\sum_{i=1}^{k} p_{i}\right) \log k,
\end{aligned}
$$

where $X_{1} \cdots, X_{k}$ denotes any representative of

$$
\left[X_{1}\right] . \cdots .\left[X_{k}\right] \in C H_{\left|X_{1}\right| \cap \ldots \cap\left|X_{k}\right|}^{*}(\mathbb{P}(E)) .
$$

This follows from a proof similar to the one of Theorem 5.4.4, using the "multijoin" $X_{1} \# \ldots \# X_{k}$ in $\mathbb{P}\left(E^{\oplus k}\right)$.

We may also consider the case of a product of projective spaces $\mathbb{P}=\prod_{i=1}^{k} \mathbb{P}\left(E_{i}\right)$, where $\bar{E}_{i}$ is an hermitian vector bundle of rank $N_{i}+1$ over $S$. For any cycle $Z$ on $\mathbb{P}$ and any multi-integer $A=\left(a_{1}, \ldots, a_{k}\right) \in \mathbb{N}^{k}$, we let

$$
h^{A}(Z)=\widehat{\operatorname{deg}}\left(\widehat{c}_{a_{1}}\left(\bar{Q}_{1}\right) \cdots \widehat{c}_{a_{k}}\left(\bar{Q}_{k}\right) \mid Z\right) \in \mathbb{R},
$$

resp.

$$
\operatorname{deg}_{K}^{A}(Z)=\operatorname{deg}_{K}\left(\widehat{c}_{a_{1}}\left(\bar{Q}_{1}\right) \cdots \widehat{c}_{a_{k}}\left(\bar{Q}_{k}\right) \mid Z\right) \in \mathbb{Z},
$$

where $\bar{Q}_{i}$ is the pull-back on $\mathbb{P}$ of the canonical hermitian quotient bundle on $\mathbb{P}\left(E_{i}\right)$. When $|A|:=a_{1}+\cdots+a_{k} \neq \operatorname{dim}(Z)$, resp. $|A| \neq \operatorname{dim}\left(Z_{K}\right)$, we have $h^{A}(Z)=0$, resp. $\operatorname{deg}_{K}^{A}(Z)=0$. Assume now that $X \in Z_{p}(\mathbb{P})$ and $Y \in Z_{q}(\mathbb{P})$ are effective cycles on $\mathbb{P}$ meeting properly on $\mathbb{P}_{K}$ and that $p+q>1+\sum_{i=1}^{k} N_{i}$. If $\mathbf{N}$ is the multi-integer $\left(N_{1}, \ldots, N_{k}\right)$, by mimicking the proof of Theorem 5.4.4 and using (2.3.19) to evaluate multiheights of external products, we get 


$$
\begin{aligned}
h^{A}(X . Y) \leq & \sum_{\substack{B, C \\
B+C=A+\mathrm{N}}}\left(h^{B}(X) \operatorname{deg}_{K}^{C}(Y)+\operatorname{deg}_{K}^{B}(X) h^{C}(Y)\right) \\
& +[K: \mathbb{Q}] \sum_{i=1}^{k}\left(N_{i}+1-\frac{p+q}{2}\right) \operatorname{deg}_{K}^{A_{i}}(X . Y) \cdot \log 2,
\end{aligned}
$$

where $A_{i}=\left(a_{1}, \ldots, a_{i-1}, a_{i}-1, a_{i+1}, \ldots, a_{k}\right)$ when $A=\left(a_{1}, \ldots, a_{k}\right)$.

Theorem 5.5.1 may also be extended to these more general situations. We leave this to the reader.

\section{Positive Green forms}

6.1. Positive Green forms and the Bézout theorem. In this last section, we shall discuss the following problem:

Given an effective cycle $Z$ on a complex variety $X$, when is it the case that $Z$ has a positive Green form, i.e., a positive form $\eta$ on $X-Z$ which is $L^{1}$ and such that $[\eta]$ is a Green current for $Z$ ?

Usually, we shall also ask that $\eta$ is of $\log$ type along $|Z|$ (see 1.1.2).

We have several reasons for asking this question. We noticed in Proposition 1.4.1 that the Levine form is positive, and this was used in Proposition 1.4.2 and Proposition 4.1.3. In Nevanlinna theory, this positivity is used to derive the Nevanlinna inequality from the first main theorem ([St4], [Sh], §4.4, (21)), so this question is a prerequisite for extending Nevanlinna theory to arbitrary varieties $([\mathrm{Co}-\mathrm{G}])$.

This question is also relevant in the content of Arakelov geometry. For instance, when $X$ is an arithmetic variety, we could say that an arithmetic cycle $(Z, g)$ on $X$ is effective when $Z$ is an effective algebraic cycle and $g=[\eta]$, where $\eta$ is a positive Green form of log type along $|Z|$. In codimension one, these are pairs $\left(\operatorname{div}(s),-\log \|s\|^{2}\right)$, where $s$ is a global section of an hermitian line bundle of sup norm less than one.

The notion of positive Green form can also be used to give another proof of the arithmetic Bézout theorem. To see that, let $N \geq 1$ be an integer, $\Delta$ be the diagonal in $\mathbb{P}^{N}(\mathbb{C}) \times \mathbb{P}^{N}(\mathbb{C}), \mu=c_{1}(\overline{\mathscr{O}(1)})$ be the standard Fubini-Study $(1,1)$ form on $\mathbb{P}^{N}(\mathbb{C}), p r_{i}: \mathbb{P}^{N}(\mathbb{C}) \times \mathbb{P}^{N}(\mathbb{C}) \rightarrow \mathbb{P}^{N}(\mathbb{C})$ be the two projections, $i=1,2$, and $\mu_{i}=p r_{i}^{*}(\mu)$. Assume that $\eta$ is a positive real form of type $(N-1, N-1)$ on $\mathbb{P}^{N}(\mathbb{C}) \times \mathbb{P}^{N}(\mathbb{C})-\Delta$, which is of log type along $\Delta$ and such that $d d^{c}[\eta]+\delta_{\Delta}$ is an harmonic form (for the standard Kähler structure on $\mathbb{P}^{N}(\mathbb{C}) \times \mathbb{P}^{N}(\mathbb{C})$, defined by $\left.\mu_{1}+\mu_{2}\right)$. For any integer $q, 0 \leq q \leq N+1$, let

$$
\lambda_{q}=\int_{\mathbb{P}^{N}(\mathbb{C}) \times \mathbb{P}^{N}(\mathbb{C})} \eta \mu_{1}^{q} \mu_{2}^{N+1-q} .
$$

Now consider the situation of 4.2 .3 and 5.4.1, i.e., let $\bar{E}$ be an hermitian vector bundle of rank $N+1$ over $S$, and let $X \in Z_{p}(\mathbb{P}(E))$ and $Y \in Z_{q}(\mathbb{P}(E))$ be effective cycles on $\mathbb{P}(E)$ which meet properly on the generic fiber, $p+q \geq$ $N+1$. 
Theorem 6.1.1. Under the above hypotheses, the following inequality holds:

$$
\begin{aligned}
h(X . Y) \leq & h(X) \operatorname{deg}_{K}(Y)+\operatorname{deg}_{K}(Y) h(X) \\
& +[K: \mathbb{Q}] c_{p q} \operatorname{deg}_{K}(X) \operatorname{deg}_{K}(Y),
\end{aligned}
$$

where

$$
c_{p q}=\frac{1}{2} \lambda_{q}+\sigma_{p-1}+\sigma_{q-1}-\sigma_{N}-\sigma_{p+q-N-2} \text { if } p+q>N+1
$$

and

$$
c_{p q}=\frac{1}{2} \lambda_{q}+\sigma_{p-1}+\sigma_{q-1}-\sigma_{N}-[K: \mathbb{Q}]^{-1} \widehat{\operatorname{deg} E} \text { if } p+q=N+1 .
$$

Proof. According to Proposition 5.4.3, (6.1.1) follows from the lower bound

$$
\int_{\mathbb{P}(E)(\mathbb{C})} \delta_{X} g_{Y} \mu^{p+q-N-1} \geq-[K: \mathbb{Q}] \lambda_{q} \operatorname{deg}_{K}(X) \operatorname{deg}_{K}(Y),
$$

where $g_{Y}$ is a $\mu$-normalized Green current for $Y$. To prove it, we may replace $\mathbb{P}(E)(\mathbb{C})$ by $\mathbb{P}^{N}(\mathbb{C})$ and consider the integral

$$
\int_{\mathbb{P}^{N}(\mathbb{C})} \delta_{X} g_{Y} \mu^{p+q-N-1},
$$

where $X$ and $Y$ are irreducible cycles on $\mathbb{P}^{N}(\mathbb{C})$ of dimensions $p-1$ and $q-1$ respectively.

Denote by $H$ the harmonic projection for the standard Kähler structures on $\mathbb{P}^{N}(\mathbb{C})$ and $\mathbb{P}^{N}(\mathbb{C}) \times \mathbb{P}^{N}(\mathbb{C})$, and let

$$
g_{\Delta}=[\eta]-H([\eta])
$$

According to formula (1.2.8) and [B1], Theorem 2.1, (i), we have

$$
g_{Y} \equiv p r_{1 *}\left(g_{\Delta} p r_{2}^{*} \delta_{Y}\right) \text {. }
$$

(Note in the proof of [B1], loc. cit., that $H\left(p r_{1 *}\left(g_{\Delta} p r_{2}^{*} \delta_{Y}\right)\right)=0$ uses Stokes' formula for currents; this is justified there by the consideration of wave front sets and, in our case, by the fact that $\eta$ is of log type along $\Delta$.) Therefore

$$
\int_{\mathbb{P}^{N}(\mathbb{C})} \delta_{X} g_{Y} \mu^{p+q-N-1}=\int_{\mathbb{P}^{N}(\mathbb{C}) \times \mathbb{P}^{N}(\mathbb{C})} p r_{1}^{*}\left(\delta_{X}\right) \cdot p r_{2}^{*}\left(\delta_{Y}\right) \cdot g_{\Delta} \mu_{1}^{p+q-N-1} .
$$

Clearly

$$
H([\eta])=\sum_{i=0}^{N} \lambda_{i} \mu_{1}^{N-i} \mu_{2}^{i-1}
$$

so that

$$
\begin{aligned}
& \int_{\mathbb{P}^{N}(\mathbb{C}) \times \mathbb{P}^{N}(\mathbb{C})} p r_{1}^{*}\left(\delta_{X}\right) \cdot p r_{2}^{*}\left(\delta_{Y}\right) \cdot H([\eta]) \mu_{1}^{p+q-N-1} \\
& =\lambda_{q}\left(\int_{\mathbb{P}^{N}(\mathbb{C})} \delta_{X} \mu^{p-1}\right)\left(\int_{\mathbb{P}^{N}(\mathbb{C})} \delta_{Y} \mu^{q-1}\right) \\
& =\lambda_{q} \operatorname{deg}(X) \operatorname{deg}(Y) .
\end{aligned}
$$


Since $\eta$ is positive, we deduce from (6.1.3), (6.1.4), and (6.1.5) that

$$
\int_{\mathbb{P}^{N}(\mathbb{C})} \delta_{X} g_{Y} \mu^{p+q-N-1} \geq-\lambda_{q} \operatorname{deg}(X) \operatorname{deg}(Y) .
$$

This proves $(6.1 .2)$.

One way to construct a positive Green form $\eta$ for the diagonal is as follows. Let $D \subset \mathbb{P}^{2 N+1}(\mathbb{C})$ be the diagonal subspace, made of points of homogeneous coordinates $\left(x_{0}, \ldots, x_{N}, x_{0}, \ldots, x_{N}\right), B \subset \mathbb{P}^{N}(\mathbb{C}) \times \mathbb{P}^{N}(\mathbb{C}) \times \mathbb{P}^{2 N+1}(\mathbb{C})$ be the set of points with homogeneous coordinates $\left(x_{0}: \ldots: x_{N}, y_{0}: \ldots: y_{N}, \lambda x_{0}\right.$ : $\left.\ldots: \lambda x_{N}: \mu y_{0}: \ldots: \mu y_{N}\right)$, where $(\lambda, \mu) \neq(0,0), q_{1}: B \rightarrow \mathbb{P}^{N}(\mathbb{C}) \times \mathbb{P}^{N}(\mathbb{C})$ and $q_{2}: B \rightarrow \mathbb{P}^{2 N+1}(\mathbb{C})$ be the projections, and $\Lambda$ be the Levine form of $D$ in $\mathbb{P}^{2 N+1}(\mathbb{C})$. Then $\eta=q_{1 *}\left(q_{2}^{*}(\Lambda)\right)$ has the required properties. One may compute

$$
\lambda_{q}=(N+1) \log (2)+2\left(\sigma_{N}-\sigma_{q}-\sigma_{N-q-1}\right) .
$$

This leads to a constant $c_{p q}$ in Theorem 6.1.1 which is bigger than the constants appearing in Theorem 4.2.3 and Theorem 5.4.4.

Remark. The argument used in the proof of Theorem 6.1.1 to get a lower bound for $g_{Y}$ applies more generally to the situation of 5.3, once there exists a positive Green form $\eta$ for the diagonal $\Delta$ in $X(\mathbb{C}) \times X(\mathbb{C})$ of log type along $\Delta$. Assume as in Proposition 5.3.1 that the cycles $Y \in Z^{p}(X)$ and $Z \in Z^{q}(X)$ meet properly on $X_{K}$ and that the product of two $\mu$-harmonic forms on $X(\mathbb{C})$ is still harmonic. Also assume that there exists an hermitian line bundle $\bar{L}$ on $X$ with first Chern form a positive multiple of the Kähler form $\mu$. Let $k=$ $\operatorname{dim}(X)-p-q$. From Proposition 5.3.1 and the proof of Theorem 6.1.1 we get the estimate

$$
\begin{aligned}
\widehat{\operatorname{deg}}\left(\hat{c}_{1}(\bar{L})^{k}[Y . Z]_{\mu}\right)-\widehat{\operatorname{deg}}\left(\hat{c}_{1}(\bar{L})^{k}[Y]_{\mu}[Z]_{\mu}\right) \\
\quad=-\frac{1}{2} \int_{X(\mathbb{C}) \times X(\mathbb{C})} g_{\Delta} p r_{2}^{*}\left(\delta_{Y}\right) p r_{1}^{*}\left(\delta_{Z} c_{1}(\bar{L})^{k}\right) \\
\quad \leq \frac{1}{2} \int_{X(\mathbb{C}) \times X(\mathbb{C})} H([\eta]) p r_{2}^{*}\left(\delta_{Y}\right) p r_{1}^{*}\left(\delta_{Z} c_{1}(\bar{L})^{k}\right)=\varphi(\operatorname{cl}(Y), \operatorname{cl}(Z)),
\end{aligned}
$$

where $\operatorname{cl}(Y) \in H^{p, p}\left(X_{\mathbb{R}}\right)$, resp. $\operatorname{cl}(Z) \in H^{q, q}\left(X_{\mathbf{R}}\right)$, denotes the cohomology class of $Y$, resp. $Z$, and $\varphi$ is some bilinear form on $H^{p, p}\left(X_{\mathbf{R}}\right) \times H^{q, q}\left(Y_{\mathbf{R}}\right)$ which depends only on $\mu, \eta$, and $L_{K}$. That type of inequality may be used to extend the arithmetic Bézout theorem to grassmannians (cf. 6.2.2, Example (iii) infra).

\subsection{Construction of positive Green forms.}

6.2.1. In this section, we use the constructions of 1.2 to produce, under suitable hypotheses, positive Green forms for effective cycles.

Proposition 6.2.1. Let $X$ be a compact Kähler manifold, and $Y \subset X$ a complex submanifold of codimension $p$. Suppose that the following two conditions are satisfied: 
(i) there exists a holomorphic vector bundle $F$ of rank $p$ over an open neighborhood $\Omega$ of $Y$ in $X$ such that $Y$ is defined by the vanishing of some holomorphic section $\sigma$ of $F$ over $\Omega$, which is transverse to the zero section;

(ii) the canonical quotient bundle $Q_{F}$ on $\mathbb{P}(F)$ may be endowed with an hermitian metric $\|$.$\| such that the top Chern form c_{p-1}\left(Q_{F},\|\|\right)$ is positive.

Then there exists a positive Green form for $Y$ (of log type along $Y$ when $X$ is projective).

Proof. Let $\nu: \widetilde{X} \rightarrow X$ be the blow-up of $Y$ in $X$. We saw in 1.2.3 that $\sigma$ defines an embedding $f: \widetilde{X} \rightarrow \mathbb{P}(F)$ and that there exists a $C^{\infty}$ form $\eta$ on $\mathbb{P}(F)$ such that

$$
g=\nu_{*}\left(\nu^{*} \log \|\sigma\|^{-2} \cdot f^{*} c_{p-1}\left(\bar{Q}_{F}\right)+f^{*}(\eta)\right)
$$

is a Green form for $Y$ in $X$ (cf. (1.2.11)). Let $\omega$ be a Kähler form on $\tilde{X}$. Since $f^{*}(\eta)$ is $C^{\infty}$ on $\tilde{X}$ and $\nu^{*} \log \|\sigma\|^{-2} \cdot f^{*} c_{p-1}\left(\bar{Q}_{F}\right)$ is positive on a neighborhood of $E=\nu^{-1}(Y)$ in $\tilde{X}$ and $C^{\infty}$ on $\widetilde{X}-E$, it follows from Proposition 1.1.4, (ii) and (iv), that if $t \in \mathbb{R}_{+}$is large enough, the current

$$
g^{\prime}=\nu_{*}\left(\nu^{*} \log \|\sigma\|^{-2} \cdot f^{*} c_{p-1}\left(\bar{Q}_{F}\right)+f^{*}(\eta)+t \omega^{p-1}\right)
$$

is a positive form on $X-Y$. On the other hand, $d d^{c} g^{\prime}=d d^{c} g$ since $\omega$ is closed. Therefore $g^{\prime}$ is a Green form for $Y$ in $X$, which is clearly of log type along $Y$ when $X$ is projective.

Examples and remarks. (i) When $Y$ is a smooth hypersurface, the hypotheses of Proposition 6.2.1 are satisfied by taking $\Omega=X$ and $F=\mathscr{O}(Y)$. In fact, any effective divisor $Z$ on any complex manifold $X$ admits a positive Green form: if \|\| is any hermitian metric on $\mathscr{O}(Z)$, if $s$ is a holomorphic section of $\mathscr{O}(Z)$ with divisor $Z$, and if $\rho: \mathbb{R}_{+} \rightarrow[0,1]$ is a $C^{\infty}$ function such that

$$
\rho_{\mid[0,1 / 2]} \equiv 1 \quad \text { and } \quad \rho_{\mid[1,+\infty[} \equiv 0 \text {, }
$$

then

$$
g=(\rho \circ\|s\|) \cdot \log \|s\|^{-2}
$$

is such a Green form.

(ii) The hypotheses of Proposition 6.2.1 are easily seen to be satisfied when $Y$ is a point. In fact, the existence of a positive Green form for any point $P$ in a complex manifold follows immediately from the positivity of the BochnerMartinelli Green form (cf. 1.2.3, Example (ii)): it is enough to pull back this Green form to a neighborhood $\Omega$ of $P$ from $\mathbb{C}^{\operatorname{dim} X}$ using holomorphic coordinates centered at $P$, and to "truncate" it by multiplication by a $C^{\infty}$ nonnegative function on $X$, supported by a small enough compact neighborhood of $P$ in $\Omega$, which takes the value 1 near $P$. Suppose that $X$ is compact, Kähler, and connected, and let $\sigma$ be a positive volume form on $X$ such that $\int_{X} \sigma=1$. By adding suitable $C^{\infty}$ forms to the Green forms obtained by this construction, we get a family $\left\{g_{P}\right\}_{P \in X}$ of positive Green forms for the points 
of $X$, which is bounded in the $L^{1}$ topology and such that, for any $P \in X$,

$$
d d^{c} g_{P}+\delta_{P}=\sigma .
$$

If $L$ is any holomorphic line bundle on $X$ equipped with an hermitian metric \|\| , the tensor powers $L^{n}, n \in \mathbb{N}$, may be endowed with the tensor power metrics and we can define "quasinorms" \|\|$_{p}, p \in[0, \infty]$, on the space of sections $H^{0}\left(X, L^{n}\right)$, by formulae (1.4.8) and (1.4.9). Then the inequalities (1.4.10) still hold, and the same argument as in the proof of Proposition 1.4.2, using $g_{P}$ instead of $\Lambda_{P}$, shows that there exists $C \in \mathbb{R}_{+}^{*}$ such that, for any $n \in \mathbb{N}$ and any $s \in H^{0}\left(X, L^{n}\right)$,

(Compare [Vo], Lemma 8.1.)

$$
\|s\|_{\infty} \leq C^{n}\|s\|_{0}
$$

(iii) Let $X$ be any complex manifold and $Y \subset X$ a complex submanifold. Assume that condition (i) in Proposition 6.2.1 is satisfied and that the restriction of $F$ to $\Omega$ is the quotient of a trivial bundle. Equip this restriction and the canonical quotient bundle $Q_{F}$ on the projective bundle $p: \mathbb{P}(F) \rightarrow \Omega$ with the quotient metric of the trivial metric. It is shown in [B-C], $\S 5$, that $c_{p-1}\left(\overline{Q_{F}}\right)$ is positive and that there exists a positive form $\eta \in A^{p-1, p-1}(\mathbb{P}(F))$, defined by local formulae in terms of the hermitian metric on $F$, such that

$$
d d^{c} \eta=p^{*} c_{p}(\bar{F})-c_{1}(\overline{\mathscr{O}(-1)}) \cdot c_{p-1}\left(\overline{Q_{F}}\right) \text {. }
$$

Then the Green form (1.2.11):

$$
g=\nu_{*}\left(\nu^{*} \log \|\sigma\|^{-2} \cdot f^{*} c_{p-1}\left(\bar{Q}_{F}\right)+f^{*}(\eta)\right),
$$

is a Green form for $Y$ in $\Omega$, positive on a neighborhood of $Y$. If $\rho \in C^{\infty}(X)$ is nonnegative, has its support in $\Omega$, and is equal to 1 in a neighborhood of $Y$, then $\rho g$ is a positive Green form for $Y$ in $X$.

Observe that the preceding hypotheses hold if $F$ is generated by its global sections on $\Omega$ (this is elementary, after shrinking $\Omega$ if necessary, when $Y$ is compact; the general assertion is proved by a standard argument using the Baire theorem). Using the construction of 1.2.3, Example (iii), it follows that any submanifold $Y$ of a Stein manifold $X$ admits a positive Green form.

6.2.2. If we combine the preceding discussion with the construction of Green forms "by reduction to the diagonal" provided by Lemma 1.2.2, we get the following statement:

Proposition 6.2.2. Suppose that a smooth projective complex variety $M$ satisfies the following condition $(C)$ :

There exists an open neighborhood (in the complex topology) $\Omega$ of the diagonal $\Delta$ in $M \times M$, a holomorphic vector bundle $E$ on $\Omega$ generated by its global holomorphic sections on $\Omega$, and a holomorphic section $\sigma$ of $E$ over $\Omega$, transverse to the zero section, which vanishes exactly on $\Delta$. Then any effective cycle $Z$ on $M$ admits a positive Green form of log type along $|Z|$.

Proof. Indeed, if $(C)$ holds, there exists a positive Green form $g_{\Delta}$ of log type for $\Delta$ by Proposition 6.2.1 and 6.2.1, Example (iii), and $g=p_{*} \mu^{*} g_{\Delta}$ is positive on $M-|Z|$ by Proposition 1.1.4, (i) and (ii). 
Examples and remarks. (i) The product of two projective varieties which satisfy $(C)$ clearly satisfies it also.

(ii) Abelian varieties are easily seen to satisfy $(C)$ : if $M$ is a $g$-dimensional complex abelian variety and if $\varphi: U \rightarrow \mathbb{C}^{g}$ is a holomorphic chart defined on an open neighborhood of the origin 0 in $M$, then condition $(C)$ is satisfied by

$$
\Omega=\left\{(y, y) \in M^{2}, x-y \in U\right\}, \quad E=\mathscr{O}_{\Omega}{ }^{\oplus g},
$$

and

$$
\sigma(x, y)=\varphi(x-y)
$$

(compare with the convolution formulae of [B1], §3.2).

(iii) Flag manifolds also satisfy $(C)$. Let indeed $M$ be the space of flags of type $\left(r_{1}, \ldots, r_{k}\right)$ in $\mathbb{C}^{n}\left(0<r_{1}<r_{2}<\cdots<r_{k}<n\right)$. Points of $M$ are sequences $\mathbf{F}=\left(F_{1}, \ldots, F_{k}\right)$ of vector subspaces of $\mathbb{C}^{n}$ such that

$$
F_{1} \subset F_{2} \subset \cdots \subset F_{k}
$$

and

$$
\operatorname{dim}_{\mathbb{C}} F_{i}=r_{i} \quad(1 \leq i \leq k) .
$$

For any $\left(\mathbf{F}, \mathbf{F}^{\prime}\right) \in M \times M^{\prime}$, let $E_{\mathbf{F}, \mathbf{F}^{\prime}}$ be the vector space of $k$-tuples $\left(f_{i}\right)_{1 \leq i \leq k}$ in $\underset{i=1}{\oplus} \operatorname{Hom}\left(F_{i}, \mathbb{C}^{n} / F_{i}^{\prime}\right)$ such that the following diagrams commute:

$$
\begin{array}{ll}
F_{i} \stackrel{f_{i}}{\longrightarrow} & \mathbb{C}^{n} / F_{i}^{\prime} \\
\downarrow & \downarrow \\
F_{i+1} \stackrel{f_{i+1}}{\longrightarrow} & \mathbb{C}^{n} / F_{i+1}^{\prime}
\end{array} \quad(1 \leq i \leq k-1) .
$$

(The right vertical arrow is the surjective map defined thanks to the inclusion $F_{i}^{\prime} \subset F_{i+1}^{\prime}$.) The family $E$ of these vector spaces is naturally endowed with a structure of algebraic vector bundles over $M \times M$. To any $m \in \operatorname{End}\left(\mathbb{C}^{n}\right)$ is associated the section $\sigma_{m}$ of $E$ which sends $\left(\mathbf{F}, \mathbf{F}^{\prime}\right) \in M \times M$ to the $k$-tuple $\left(f_{i}\right)_{1 \leq i \leq k}$ defined by the composite maps

$$
f_{i}: F_{i} \longrightarrow \mathbb{C}^{n} \stackrel{m}{\longrightarrow} \mathbb{C}^{n} \longrightarrow \mathbb{C}^{n} / F_{i}^{\prime}
$$

These sections of $E$ are regular and generate $E$. Finally, the subscheme of $M \times M$ defined by $\sigma_{i d}=0$ is easily seen to coincide with $\Delta$. This shows $(C)$ is satisfied by the vector bundle $E$ on $\Omega=M \times M$ and $\sigma=\sigma_{i d}$.

(iv) Using 6.2.1, Example (iii), and a "reduction to the diagonal" analogous to Lemma 1.2.2, one may prove that any effective cycle on a Stein manifold admits a positive Green form.

6.2.3. Let us recall that a complex manifold $M$ is called homogeneous if the group $\operatorname{Aut}(M)$ of automorphisms of $M$ (as a complex manifold) acts transitively on $M$. The following facts are well known: (i) A compact complex manifold $M$ is homogeneous iff there exist a complex Lie group $G$ and a 
closed complex subgroup $H$ of $G$ such that $M \simeq G / H$. Moreover, if $M$ is projective, $G$ may be chosen to be a complex algebraic group, and the action $G \times M \rightarrow M$ of $G$ on $M$ a morphism of algebraic varieties.

(ii) A compact connected complex manifold $M$ is homogeneous iff its holomorphic tangent bundle $T_{M}$ is generated by its global holomorphic sections over $M$. In particular, any connected smooth projective variety $M$ which satisfies condition $(C)$ of Proposition 6.2.2 is homogeneous (indeed, if $E$ and $\sigma$ are as in $(C)$, the differential of $\sigma$ along $\Delta$ defines an isomorphism from $T_{M} \simeq N_{\Delta}(M \times M)$ to $E_{M}$, which is generated by its global sections).

For more information on compact homogeneous complex manifolds, see [B$\mathrm{R}$ ] and the references given there. Let us only mention that the connected compact homogeneous algebraic complex manifolds are projective (Chow) and are exactly the products of complex abelian varieties and generalized flag manifolds ${ }^{5}$ (Borel-Remmert).

One may ask if any effective cycle on such a manifold admits a Green form of log type. (According to the examples in 6.2.2, this is true for any product of abelian varieties and flag manifolds.) The following proposition, due to O. Gabber, solves a variant of this question.

Proposition 6.2.3. Any effective cycle $Z$ on a compact homogeneous complex manifold $M$ admits a positive Green form.

Proof. Clearly we may assume that $Z$ is irreducible, and consider a resolution of singularities $\nu: \tilde{Z} \rightarrow Z$. Let $G$ be a complex Lie group acting transitively on $M$ (cf. (i) above), and let $f: G \times \tilde{Z} \rightarrow M$ be the holomorphic smooth map defined by

$$
f(g, x)=g \cdot \nu(x) .
$$

The identity element $e$ of $G$, seen as a 0 -cycle in $G$, admits a positive Green form $g_{e}$ with compact support (6.2.1, Example (ii)). Let $\omega=d d^{c} g_{e}+\delta_{e}$. If $p r: G \times \tilde{Z} \rightarrow G$ denotes the first projection, the current $p r^{*} g_{e}$ is compactly supported, and

$$
g=f_{*} p r^{*} g_{e}
$$

is well defined. Moreover

$$
d d^{c} g=f_{*} p r^{*} d d^{c} g_{e}=f_{*} p r^{*}\left(\omega-\delta_{e}\right)=f_{*} p r^{*} \omega-f_{*} \delta_{e \times \tilde{Z}}
$$

We have

$$
f_{*} \delta_{e \times \tilde{Z}}=\nu_{*} \delta_{\tilde{Z}}=\delta_{Z}
$$

On the other hand, since $f$ is smooth and $p r^{*} \omega$ is $C^{\infty}$ and compactly supported, $f_{*} p r^{*} \omega$ is $C^{\infty}$. This shows that $g$ is a Green current for $Z$ in $M$. It is $C^{\infty}$ on $M-Z$, since $p r^{*} g_{e}$ is $C^{\infty}$ on $(G-\{e\}) \times Z$, which contains $f^{-1}(M-Z)$, and it is positive by Proposition 1.1 .4 , (i) and (ii).

Remark. Let $X$ be a smooth quasi-projective complex variety and $Y$ a proper closed algebraic subset of $X$. We can say that a $C^{\infty}$ form $\eta$ on $X-Y$

\footnotetext{
${ }^{5}$ A generalized flag manifold is a quotient $G / P$, where $G$ is a connected reductive complex algebraic group and $P$ a parabolic subgroup of $G$.
} 
is "almost of log type" along $Y$ when the conditions in Definition 1.1.1 are satisfied, except that only the restriction of $\pi$ to the support of $\varphi$, and not $\pi$ itself, is supposed to be proper. Observe that the Green form $g$ constructed in the preceding proof is almost of log type along $|Z|$ when $M$ is projective, provided $G$ is chosen to be an algebraic group acting algebraically on $M$ and $g_{e}$ is obtained from the Bochner-Martinelli form as in 6.2.1, Example (ii).

6.3. An obstruction to the existence of positive Green forms. When the construction in the preceding section of positive Green forms for some smooth submanifolds $Y$ of a complex manifold $X$ applies, the top Chern class of the canonical quotient bundle $Q$ on the projective bundle $\mathbb{P}\left(N_{Y} X\right)$ may be represented by a closed positive form. In this section we shall see that, conversely, at least in the linear situation where $X$ is the total space of a vector bundle over $Y$, the existence of a positive Green form for $Y$ in $X$ implies a kind of "numerical effectivity" for the top Chern class of $Q$.

Let $Y$ be a smooth projective complex variety, and let $F$ be a rank $r$ vector bundle on $Y$. Consider the projective completion $X=\mathbb{P}(F \oplus \mathscr{O})$ of $F$; it contains as subvarieties $Y$ (identified with the zero section of $F$ ) and $\mathbb{P}(F)=$ $X-F$. Consider also $Q$ the canonical quotient bundle on $\mathbb{P}(F), c_{r-1}(Q)$ its top Chern class, and $p: X-Y \rightarrow \mathbb{P}(F)$ the morphism defined by the first projection $F \oplus \mathscr{O} \rightarrow F$. Finally, for any $\lambda \in \mathbb{C}^{*}$, let $\varphi_{\lambda}$ be the automorphism of $X$ which extends multiplication by $\lambda$ on the fibers of $F$.

Proposition 6.3.1. Let $g$ be any Green current for $Y$ in $X$ which is $C^{\infty}$ on $X-Y$. For any subvariety $Z$ of dimension $r-1$ in $X$, such that

$$
Z \not \subset \mathbb{P}(F) \text { and } Z \cap Y=\varnothing,
$$

the following asymptotic formula holds:

$$
\lim _{\lambda \rightarrow 0}\left(\log |\lambda|^{-1}\right)^{-1} \int_{X} g \cdot \delta_{\varphi_{\lambda}(Z)}=2\left\langle p^{*} c_{r-1}(Q),[Z]\right\rangle
$$

Related asymptotic formulae have been announced independently in [H-W]. Proof. Let $g$ and $g^{\prime}$ be two Green currents for $Y$ which are $C^{\infty}$ on $X-Y$. There exist $u \in \mathscr{D}^{r-1, r}(X), v \in \mathscr{D}^{r, r-1}(X)$, and $\psi \in A^{r, r}(X)$ such that $u$ and $v$ are $C^{\infty}$ on $X-Y$ and

$$
g^{\prime}-g=\partial u+\bar{\partial} v+\psi
$$

(see [G-S2], 1.2.2, and [B1], Proposition 1.1, (ii)). By Stokes formula, this implies that for any $\lambda \in \mathbb{C}^{*}$ :

$$
\int_{X} g^{\prime} \cdot \delta_{\varphi_{\lambda}(Z)}-\int_{X} g . \delta_{\varphi_{\lambda}(Z)}=\int_{X} \psi \cdot \delta_{\dot{\varphi}_{\lambda}(Z)}
$$

According to Corollary 1.5.2 applied to the cycle in $X \times \mathbb{A}_{\mathbb{C}}^{1}$ defined as the closure of

$$
\left\{\left(\varphi_{\lambda}(x), \lambda\right) ; x \in Z, \lambda \in \mathbb{C}^{*}\right\}
$$

which is flat over $\mathbb{A}_{\mathbb{C}}^{1}$ and therefore fulfills the hypothesis of [loc.cit.], the last integral defines a continuous function of $\lambda \in \mathbb{C}^{*}$ which extends continuously 
to $\mathbb{C}$. Therefore, to prove Proposition 6.3.1, we can assume that $g$ is a Green current obtained by the construction of $\S 1.2$. More precisely, let us choose $g$ as follows. Let $\nu: \widetilde{X} \rightarrow X$ be the blow-up of $Y$ in $X$, and let $E=\nu^{-1}(Y)$ be the exceptional divisor; we shall identify (by $\nu$ ) $\tilde{X}-E$ and $X-Y$. The map $p: X-Y \rightarrow \mathbb{P}(F)$ extends to a regular map from $\tilde{X}$ to $\mathbb{P}(F)$, which we shall still denote by $p$. In fact $p_{\mid E}$ is the canonical isomorphism from $E$ to $\mathbb{P}(F)=\mathbb{P}\left(N_{Y} X\right)$. Let us choose a metric $\|$.$\| on \mathscr{O}(E)$ and a closed form $\epsilon \in A^{r-1, r-1}(\mathbb{P}(F))$ whose cohomology class is $c_{r-1}(Q)$. According to 1.2 .2 , if $s$ is a section of $\mathscr{O}(E)$ of divisor $E$, there exists $\gamma \in A^{r-1, r-1}(\tilde{X})$ such that $g=\nu_{*}\left(\log \|s\|^{-2} \cdot p^{*} \epsilon+\gamma\right)$ is a Green current for $Y$.

To study the asymptotics of $\int_{X} g . \delta_{\varphi_{\lambda}(Z)}$ as $\lambda \rightarrow 0$, we choose a metric $\|$. on $F$ and a $C^{\infty}$ function $\psi: R_{+} \rightarrow[0,1]$ such that

$$
\psi_{\mid[0,1 / 2]}=1 \text { and } \psi_{\mid[1,+\infty[}=0 \text {, }
$$

we define $r: \tilde{X}-\mathbb{P}(F) \rightarrow \mathbb{R}_{+}$as the map which sends a point $x$ to the norm $\|\nu(x)\|$ of the element $\nu(x)$ of $F$, and we let $\rho=\psi \circ r$. Then $\frac{\|s\|}{r}$, defined on $\widetilde{X}-(E \cup \mathbb{P}(F))$, is easily seen to extend to a $C^{\infty}$ nonvanishing function on a neighborhood of $E$. Therefore there exists $\gamma^{\prime} \in A^{r-1, r-1}(\widetilde{X})$ such that

$$
\log \|s\|^{-2} \cdot p^{*} \epsilon+\gamma=\rho \cdot \log r^{-2} \cdot p^{*} \epsilon+\gamma^{\prime},
$$

and we get

$$
\int_{X} g \cdot \delta_{\varphi_{\lambda}(Z)}=\int_{\widetilde{X}} \rho \cdot \log r^{-2} \cdot p^{*} \epsilon \delta_{\varphi_{\lambda}(Z)}+\int_{\widetilde{X}} \gamma^{\prime} \cdot \delta_{\varphi_{\lambda}(Z)} .
$$

According to Corollary 1.5.2 applied to the cycle in $\tilde{X} \times \mathbb{A}_{\mathbb{C}}^{1}$ defined as the closure of $\left\{\left(\varphi_{\lambda}(x), \lambda\right) ; x \in Z, \lambda \in \mathbb{C}^{*}\right\}$, the last integral in (6.3.2) defines a continuous function of $\lambda \in \mathbb{C}^{*}$ which extends continuously to $\mathbb{C}$.

On the other hand, if $\tilde{\varphi}_{\lambda}$ denotes the automorphism of $\widetilde{X}$ which lifts $\varphi_{\lambda}$, we have $\tilde{\varphi}_{\lambda}^{*} r=|\lambda| r$ and $\tilde{\varphi}_{\lambda}^{*} p^{*} \epsilon=p^{*} \epsilon$. Therefore

$$
\begin{aligned}
\int_{\widetilde{X}} J \rho \cdot \log r^{-2} \cdot p^{*} \epsilon \cdot \delta_{\varphi_{\lambda}(Z)} & =\int_{\widetilde{X}} \tilde{\varphi}_{\lambda}^{*}\left(\rho \cdot \log r^{-2} \cdot p^{*} \epsilon\right) \delta_{Z} \\
& =\int_{\widetilde{X}} \tilde{\varphi}_{\lambda}^{*} \rho \cdot\left(\log |\lambda|^{-2}+\log r^{-2}\right) \cdot p^{*} \epsilon \cdot \delta_{Z} .
\end{aligned}
$$

The function $\log r^{-2}$ is $L^{1}$ with respect to the measure $p^{*} \epsilon . \delta_{Z}$ (indeed $\log r^{-2}$ is a function of $\log$ type along $\mathbb{P}(F)$, which meets $Z$ properly). Since $\left|\tilde{\varphi}_{\lambda}^{*} \rho\right| \leq 1$ and

$$
\lim _{\lambda \rightarrow 0} \tilde{\varphi}_{\lambda}^{*} \rho(x)=\lim _{\lambda \rightarrow 0} \varphi(\lambda r(x))=1
$$

for any $x \in X-\mathbb{P}(F)$, Lebesgue's theorem on dominated convergence shows that

$$
\int_{\widetilde{X}} \tilde{\varphi}_{\lambda}^{*} \rho \cdot \log r^{-2} \cdot p^{*} \epsilon \cdot \delta_{Z}
$$


has a limit when $\lambda \rightarrow 0$ and that

$$
\lim _{\lambda \rightarrow 0} \int_{\widetilde{X}} \tilde{\varphi}_{\lambda}^{*} \rho \cdot p^{*} \epsilon \cdot \delta_{Z}=\int_{\widetilde{X}} p^{*} \epsilon \cdot \delta_{Z}=\left\langle p^{*} c_{r-1}(Q),[Z]\right\rangle .
$$

Finally when $\lambda \rightarrow 0$, the integral (6.3.2) equals

$$
2 \log |\lambda|^{-1} \cdot\left\langle p^{*} c_{r-1}(Q),[Z]\right\rangle+O(1)
$$

From Proposition 6.3.1, we immediately get:

Corollary 6.3.2. If there exists a subvariety $Z$ of dimension $r-1$ in $X$ meeting $\mathbb{P}(F)$ and $Y$ properly such that

$$
\left\langle p^{*} c_{r-1}(Q),[Z]\right\rangle<0,
$$

then there is no Green form for $Y$ which is positive on $X-Y$.

Examples of vector bundles $F \rightarrow Y$ for which such a $Z$ exists are easily constructed. For instance, take for $Y$ any smooth projective curve, and for $F$ any rank two vector bundle which admits a subbundle $L$ of rank 1 such that

$$
\operatorname{deg} F<\operatorname{deg} L \leq-g \text {, }
$$

where "deg" denotes the degree of vector bundles on $Y$ and $g$ the genus of $Y$. Then the dual of $L$ has a nonzero regular section $s$. Its inverse $s^{-1}$ is a meromorphic section of $L$ which does not vanish, and defines a section of the projection morphism $X \rightarrow Y$. Clearly its image $Z$ does not meet $Y$ and is not contained in $\mathbb{P}(F)$. Moreover, the cycle $p_{*} Z$ is the image of the section $\sigma$ of $\mathbb{P}(F)$ defined by the subbundle $L \subset F$; since $\sigma^{*} Q \simeq F / L$, we get:

$$
\begin{aligned}
\left\langle p^{*} c_{r-1}(Q),[Z]\right\rangle & =\left\langle c_{r-1}(Q), p_{*}[Z]\right\rangle=\left\langle\sigma^{*} c_{1}(Q) ;[Y]\right\rangle \\
& =\left\langle c_{1}(F)-c_{1}(L) ;[Y]\right\rangle=\operatorname{deg} F-\operatorname{deg} L<0 .
\end{aligned}
$$

\section{REFERENCES}

[A-K] A. B. Altman and S. L. Kleiman, Joins of schemes, linear projections, Compositio Math. 31 (1975), 309-343.

[Ar1] S. J. Arakelov, Intersection theory of divisors on an arithmetic surface, Math. USSR Izv. 8 (1974), 1167-1180.

[Ar2] _ Theory of intersections on an arithmetic surface, Proc. Internat. Congr. Math., Vancouver, vol. 1, 1975, pp. 405-408.

[B-B-E-M] B. Beauzamy, E. Bombieri, P. Enflo, and H. Montgomery, Products of polynomials in many variables, J. Number Theory 36 (1990), 219-245.

[Bi-V] J.-M. Bismut and E. Vasserot, The asymptotic of the Ray-Singer analytic torsion associated with high powers of a positive line bundle, Comm. Math. Phys. 125 (1989), 355-367.

[B1] A. Blanchard, Sur les variétés analytiques complexes, Ann. Sci. École Norm. Sup. 73 (1956), 157-202.

[Bo-V] E. Boda and W. Vogel, On a system of parameters, local intersection multiplicity and Bézout's theorem, Proc. Amer. Math. Soc. 78 (1980), 1-7.

[Bo-Va] E. Bombieri and J. Vaaler, On Siegel's lemma, Invent. Math. 73 (1983), 11-32.

[B-R] A. Borel and R. Remmert, Über kompakte homogene Kählersche Mannigfaltigkeiten, Math. Ann. 145 (1962), 429-439. 
[B1] J.-B. Bost, Green's currents and height pairing on complex tori, Duke Math. J. 61 (1990), 899-912.

[B2] — Théorie de l'intersection et théorème de Riemann-Roch arithmétiques, Séminaire Bourbaki n ${ }^{0}$ 731, 1990/1991, Astérisque 201-203 (1991), 43-88.

[BGS] J.-B. Bost, H. Gillet, and C. Soulé, Une analogue arithmétique du théorème de Bézout, C. R. Acad. Sci. Paris Sér. I Math. 312 (1991), 845-848.

[B-C] R. Bott and S. S. Chern, Hermitian vector bundles and the equidistribution of zeroes of their holomorphic sections, Acta Math. 114 (1968), 71-112.

[C-Wa] W.-L. Chow and B. L. van der Waerden, Zur algebraischen Geometrie IX. Über zugeordnete Formen und algebraische Systeme von algebraischen Mannigfaltigkeiten, Math. Ann. 113 (1937), 692-704.

[Co-G] C. Cornalba and P. Griffiths, Analytic cycles and vector bundles on non-compact algebraic varieties, Invent. Math. 28 (1975), 1-206.

[Fa1] G. Faltings, Calculus on arithmetic surfaces, Ann. of Math. (2) 119 (1984), 387-424.

[Fa2] , Diophantine approximation on Abelian varieties, Ann. of Math. (2) 133 (1991), 549-576.

[Fa3] Lectures on the arithmetic Riemann-Roch theorem, notes by S. Zhang, Ann. of Math. Stud., no. 127, Princeton Univ. Press, Princeton, NJ, 1992.

[Fa-W] G. Faltings, G. Wüstholz, et al., Rational points, Aspects of Mathematics, vol. E6, Vieweg-

Verlag, Braunschweig, 1984.

[Fo] J. Fogarty, Truncated Hilbert functors, J. Reine Angew. Math. 234 (1969), 65-88.

[Fu1] W. Fulton, Rational equivalence on singular varieties, Inst. Hautes Études Sci. Publ. Math. 45 (1975), 147-167.

[Fu2] Intersection theory, Ergeb. Math. Grenzgeb. (3), Band 2, Springer-Verlag, Berlin, Heidelberg, and New York, 1984.

[Fu3] _ Introduction to intersection theory in algebraic geometry, CBMS Regional Conf. Ser. in Math., vol. 54, Amer. Math. Soc., Providence, RI, 1984.

[Ga] F. Gaeta, Sul calculo effectivo della forma associata $F\left(W_{\alpha+\beta-n}^{g \ell}\right)$ all'intersezione de duo cicle effetivi pure $U_{\alpha}^{g}, V_{\beta}^{\ell}$ di $S_{n}$ in funzione delle $F\left(U_{\alpha}^{g}\right), F\left(V_{\beta}^{\ell}\right)$ relative ai cicli secanti, Atti Accad. Naz. Lincei (8) 24 (1958), 269-276.

[Ge] I. M. Gelfand, Collected papers (S. G. Gindikin, V. W. Guillemin, A. A. Kirillov, B. Kostant, and S. Sternberg, eds.), Springer-Verlag, Berlin, 1987-1989.

[Gi] H. Gillet, An introduction to higher dimensional Arakelov theory, Contemp. Math., vol. 67, Amer. Math. Soc., Providence, RI, 1987, pp. 209-288.

[G-S1] H. Gillet and C. Soulé, Intersection theory using Adams operations, Invent. Math. 90 (1987), 243-277.

[G-S2] _ Arithmetic intersection theory, Inst. Hautes Études Sci. Publ. Math. 72 (1990), 94-174.

[G-S3] _ Characteristic classes for algebraic vector bundles with Hermitian metric, I, II, Ann. of Math. (2) 131 (1990), 163-203, 205-238.

[G-S4] — Amplitude arithmétique, C. R. Acad. Sci. Paris Sér. I Math. 307 (1988), 887-890.

[G-S5] , Un théorème de Riemann-Roch-Grothendieck arithmétique, C. R. Acad. Sci. Paris Sér. I Math. 309 (1989), 929-932.

[G-S6] _ An arithmetic Riemann-Roch Theorem, Invent. Math. 110 (1992), 473-543.

[G-S7] _ On the number of lattice points in convex symmetric bodies and their duals, Israel J. Math. 74 (1991), 347-357.

[Gr] D. R. Grayson, Reduction theory using semistability, Comment. Math. Helv. 59 (1984), 600-634.

[Gu] W. Gubler, Höhentheorie, Dissertation, ETH, Zurich, 1992. 
[H-W] B. Harris and B. Wang, Archimedean height pairing of intersecting cycles, Internat. Math. Research Notices 4 (1993), 107-111.

[Ha-K] R. Harvey and A. W. Knapp, Positive $(p, p)$ forms, Wirtinger's inequality, and currents, Value Distribution Theory (Proc. Tulane Univ. Program, 1972-73), Part A (R. O. Kujala and A. L. Vitter, eds.), Dekker, New York, 1974, pp. 43-62.

[He] S. Helgason, Groups and geometric analysis, Academic Press, New York, 1984.

[Hi] M. Hindry, Letter to J.-B. Bost, January 1990.

[K] J. R. King, The currents defined by analytic varieties, Acta Math. 127 (1971), 186-220.

[K-M] F. Knudsen and D. Mumford, The projectivity of the moduli space of stable curves I, Preliminaries on det aud div, Math. Scand. 39 (1976), 19-55.

[Kr] J. Kramer, Néron-Tate height for cycles on Abelian varieties (d'après Faltings), preprint.

[Kro] L. Kronecker, Grundzüge einer arithmetischen Theorie der algebraischen Grössen, Crelle J. Reine Angew. Math. 92 (1881-1882), 1-122; Werke, Vol. II, Leipzig, Teubner, 1897, pp. 237-287.

[La] S. Lang, Introduction to Arakelov theory, Springer-Verlag, Berlin, Heidelberg, and New York, 1988.

[Lau] M. Laurent, Hauteur de matrices d'interpolation, Approximations Diophantiennes et Nombres Transcendants (Luminy 1990) (P. Philippon, ed.), de Gruyter, Berlin, 1992, pp. 215-238.

[Le1] P. Lelong,Plurisubharmonic functions and positive differential forms, Gordon and Breach, New York, 1969.

[Le2] _ Mesure de Mahler des polynômes et majoration par convexité, C.R. Acad. Sci. Paris 315 (1992), 139-142.

[Lev] H. Levine, A theorem on holomorphic mappings into complex projective space, Ann. of Math. (2) 71 (1960), 529-535.

[MB1] L. Moret-Bailly, Pinceaux de variétés abéliennes, Astérisque 129 (1985).

[MB2] _ Métriques permises, Séminaire sur les Pinceaux Arithmétiques; la Conjecture de Mordell, Astérisque 127 (1985), 29-87.

[MB3] _ Hauteurs et classes de Chern sur les surfaces arithmétiques, Astérisque 183 (1990), $37-58$.

[Me] F. Mertens, Über die bestimmenden Eigenschaften der Resultante von n-Formen mit $n$ Veränderlichen, Sitzungsber. Wiener Akad. 93 (1886), 527-566.

[M-F] D. Mumford and J. Fogarty, Geometric invariant theory, Second ed., Springer-Verlag, Berlin, 1982.

[N1] Yu. V. Nesterenko, Estimates for the orders of zeros of functions of a certain class and their applications in the theory of transcendental numbers, Izv. Akad. Nauk. USSR Ser. Mat. 41 (1977); English transl., Math. USSR-Izv. 11 (1977), 239-270.

[N2] Estimates for the characteristic function of a prime ideal, Mat. Sbornik 123 (1984); English transl., Math. USSR-Sb. 51 (1985), 9-32.

[No] D. G. Northcott, An inequality in the theory of arithmetic on algebraic varieties, Proc. Cambridge Philos. Soc. 45 (1949), 502-509.

[P1] P. Philippon, Critères pour l'indépendance algébrique, Inst. Hautes Études Sci. Publ. Math. 64 (1986), 5-52.

[P2] _ Sur des hauteurs alternatives I, Math. Ann. 289 (1991), 255-283.

[P3] _ Sur des hauteurs alternatives II, preprint, 1991.

[Sa] P. Samuel, Méthodes d'algèbre abstraite en géométrie algébrique, Second ed., SpringerVerlag, Berlin, 1967.

[Sch] W. M. Schmidt, On heights of algebraic subspaces and diophantine approximations, Ann. of Math. (2) 85 (1967), 430-472.

[Sh] B. V. Shabat, Distribution values of holomorphic mappings, Transl. Math. Mono., vol. 61, Amer. Math. Soc., Providence, RI, 1985. 
[Si] C. L. Siegel, Über einige Anwendungen diophantischer Approximationen, Abhandlungen Preussischen Akad. Wiss. (1929), 41-69.

[So1] C. Soulé, Théorie de Nevanlinna et théorie d'Arakelov, Astérisque 183 (1990), 127-135.

[So2] - Géométrie d'Arakelov et théorie des nombres transcendants, J. Arithmétiques de Luminy (17-21 juillet 1989), Astérisque 198-200 (1991), 355-371.

[So3] _ Opérations en K-théorie algébrique, Canad. J. Math. 27 (1985), 488-550.

[So-A-B-K] C. Soulé, D. Abramovich, J.-F. Burnol, and J. Kramer, Lectures on Arakelov geometry, Cambridge Stud. Adv. Math., vol. 33, Cambridge Univ. Press, Cambridge, 1992.

[St1] W. Stoll, The continuity of the fiber integral, Math. Z. 95 (1967), 87-138.

[St2] About the value distribution of holomorphic maps into projective space, Acta Math. 123 (1969), 83-114.

[St3] _ Value distribution of holomorphic maps into compact complex manifolds, Lecture Notes in Math., vol. 135, Springer-Verlag, Berlin, Heidelberg, and New York, 1970.

[St4] _ Aspects of value distribution theory in several complex variables, Bull. Ann. Math. Soc. 83 (1977), 166-183.

[Stu] U. Stuhler, Eine Bemerkung zur Reduktionstheorie quadratischen Formen, Arch. Math. 27 (1976), 604-610.

[S-V] T. Struppeck and J. D. Vaaler, Inequalities for heights of algebraic subspaces and the Thue-Siegel principle, Analytic Number Theory (B. C. Berndt, H. G. Diamond, H. Halberstam, and A. Hildegrand, eds.), Progr. Math., vol. 85, Birkhäuser, Boston, 1983, pp. 493-527.

[Sz] L. Szpiro, Degrés, intersections, hauteurs, Séminaire sur les Pinceaux Arithmétiques, la Conjecture de Mordell, Astérisque 127 (1985), 11-28.

[V] W. Vogel, Lectures on results on Bézout's theorem, Tata Inst. Fund. Res., Bombay, 1984.

[Vo] P. Vojta, Applications of arithmetic algebraic geometry to diophantine approximations, CIME Proceedings Trento, 1991 (to appear).

[Wa] B. L. van der Waerden, Moderne algebra II, zweite verbesserte Auflage, Springer, Berlin, 1940.

[We1] A. Weil, Number-theory and algebraic geometry, Vol. II, Proc. Internat. Math. Congress, Cambridge, MA, 1950, pp. 90-100.

[We2] _ Arithmetic on algebraic varieties, Ann. of Math. (2) 53 (1951), 412-444.

[Wu] G. Wüstholz, Uber das abelsche Analogen des Lindemannschen Satzes, Invent. Math. 72 (1983), 363-388.

[Zh1] S. Zhang, Positive line bundles on arithmetic surfaces, Ann. of Math. (2) 136 (1992), 569-587.

[Zh2] _ Positive line bundles on arithmetic varieties, preprint, February 1992.

ABSTRACT. Using arithmetic intersection theory, a theory of heights for projective varieties over rings of algebraic integers is developed. These heights are generalizations of those considered by Weil, Schmidt, Nesterenko, Philippon, and Faltings. Several of their properties are proved, including lower bounds and an arithmetic Bézout theorem for the height of the intersection of two projective varieties. New estimates for the size of (generalized) resultants are derived. Among the analytic tools used in the paper are "Green forms" for analytic subvarieties, and the existence of positive Green forms is discussed.

(J.-B.Bost and C. Soulé) Institut des Hautes Études Scientifiques, 35, Route de Chartres, 91440, BURES-SUR-YveTtes, FRANCE

(H. Gillet) Department of Mathematics, Statistics, and Computer Science, (m/c249), University of Illinois at Chicago, 851 S. Morgan Street, Chicago, Illinois 60607

E-mail address: henri@math.uic.edu 